\title{
IMMUNE RESPONSES IN PERFORIN DEFICIENT MICE
}

\author{
By \\ Helen Mary Alys Simkins
}

\begin{abstract}
A thesis
submitted to the Victoria University of Wellington in fulfilment of the requirements for the degree of Doctor of Philosophy in Cell and Molecular Biology
\end{abstract}

Victoria University of Wellington

2009 


\section{Abstract}

Dendritic cells (DC) play a pivotal role in the initiation of $\mathrm{T}$ cell responses and earlier studies have shown that their survival is important for the generation of effective immune responses. Cytotoxic T lymphocytes (CTL) and natural killer T (NKT) cells have been proposed to regulate the survival of antigen presenting DC through their ability to kill cells expressing specific antigen via secretion of perforin, a protein contained in cytotoxic granules. Perforin knockout (PKO) mice generate amplified immune responses to DC immunization, suggesting a link between defective cytotoxicity and increased $\mathrm{T}$ cell responses. The studies in this thesis used PKO mice and in vivo models of $\mathrm{CD} 8^{+} \mathrm{T}$ cells and NKT cell immune responses to determine whether CTL and NKT cells eliminate DC in a perforin-dependent manner, and whether DC elimination is a mechanism to regulate $\mathrm{T}$ cell responses.

During a primary influenza infection C57BL/6 and PKO mice generated a similar influenza specific $\mathrm{CD} 8^{+}$immune response. No significant difference in the percentage of influenza epitope $\mathrm{PA}_{224-233}$ specific T cells was observed between C57BL/6 and PKO mice during a secondary influenza infection, but PKO mice had a significantly reduced $\mathrm{T}$ cell response directed towards the dominant influenza epitope, $\mathrm{NP}_{366-374}$. The reduced $\mathrm{T}$ cell response in PKO mice was not due to differences in activation or differentiation status of specific $\mathrm{T}$ cells compared to C57BL/6 mice. Therefore, the extended DC survival in PKO after secondary influenza viral infection, recently reported by other authors, does not appear to correlate with increased expansion of virus specific $\mathrm{CD}^{+} \mathrm{T}$ cells in infected mice.

The role of NKT cells in DC elimination was assessed in vivo using the NKT cell ligand $\alpha$ Galactosylceramide ( $\alpha$-GalCer). Injection of $\alpha$-GalCer in C57BL/6 mice induced a dramatic decline in the number of splenic $\mathrm{CD}^{+} \mathrm{DC}$. A similar decrease in $\mathrm{CD}^{+} \mathrm{DC}$ numbers was observed in PKO mice, suggesting that the mechanism of DC loss did not involve perforinmediated killing. In contrast, treatment with a TNF- $\alpha$ neutralizing antibody substantially reduced the decline in $\mathrm{CD} 8^{+} \mathrm{DC}$ numbers. This reduction in splenic $\mathrm{CD} 8^{+} \mathrm{DC}$ occurred as early as $15 \mathrm{hr}$ after $\alpha$-GalCer treatment, and did not affect generation of $\mathrm{CD}^{+} \mathrm{T}$ cell responses or the ability of $\alpha$-GalCer treatment to provide tumour protection. 
Taken together, these results suggest that multiple cells and mechanisms can regulate DC survival in vivo. CTL regulate DC survival in vivo in a perforin-dependent manner, but this does not necessarily affect the magnitude of the resulting immune responses. NKT cells also affect the survival of DC in vivo, but in a perforin-independent, cytokine-dependent manner. These findings provide additional knowledge about the in vivo involvement of perforin in regulating DC survival by CTL and NKT cells and the effects this has on T cell responses. 


\section{Acknowledgements}

Firstly, I would like to thank my supervisor, Dr Franca Ronchese, for all her help and support throughout my $\mathrm{PhD}$ project, particularly for her patience during writing of the thesis. Thank you to my co-supervisor, Dr Joanna Kirman for always being positive and offering her support and proof reading when required. I also thank Dr Ian Hermans for his assistance and advice in the $\alpha$-GalCer work and Dr Margaret Baird for providing me with the PR8 virus.

I would like to thank Rachel Perret, Kathryn Farrand, Kylie Quinn and Lisa Connor for teaching me basic immunology techniques and assisting me in the lab, as well as their good company and friendship throughout my PhD. I also want to specially thank Nina Dickgreber not only for her help in the lab but also for her great friendship and fun times we had over the last 3 years. Nina, I am not sure I would have survived without you so thanks for everything!

Thank you to Kate Andrew for her help with the RT-PCR, Mark Yang and the rest of the Cancer Immunotherapy group for their support in the Lab. I also want to thank the Biological Research Unit for their animal husbandry for without I would not have been able to carry out all my experiments, and of course all the mice volunteers.

I want to thank all the staff at the Malaghan Institute for their assistance both scientific and otherwise. In particular I would like to thank Dr Jacquie Harper for her after work chats in the tea room which kept me going during the writing part of my $\mathrm{PhD}$ and to the past staff member Dr Patries Herst for her proof reading. As well I would like to thank my fellow PhD students, both past and current for their advice, discussions and generally making my time at the Malaghan enjoyable.

I must also say a big thanks to all my friends outside the Malaghan Institute who have endeavoured to support me in whatever way they could throughout the highs and lows of my $\mathrm{PhD}$. 
I am very thankful to the Lotteries health who awarded me a PhD scholarship to undertake this research and to Victoria University for their completion scholarship, which helped me towards the end of my study.

Last but certainly not least, I would like to thank my parents and family for their continued support and encouragement over the last few years and for always being proud of what I achieve. 


\section{Table of Content}

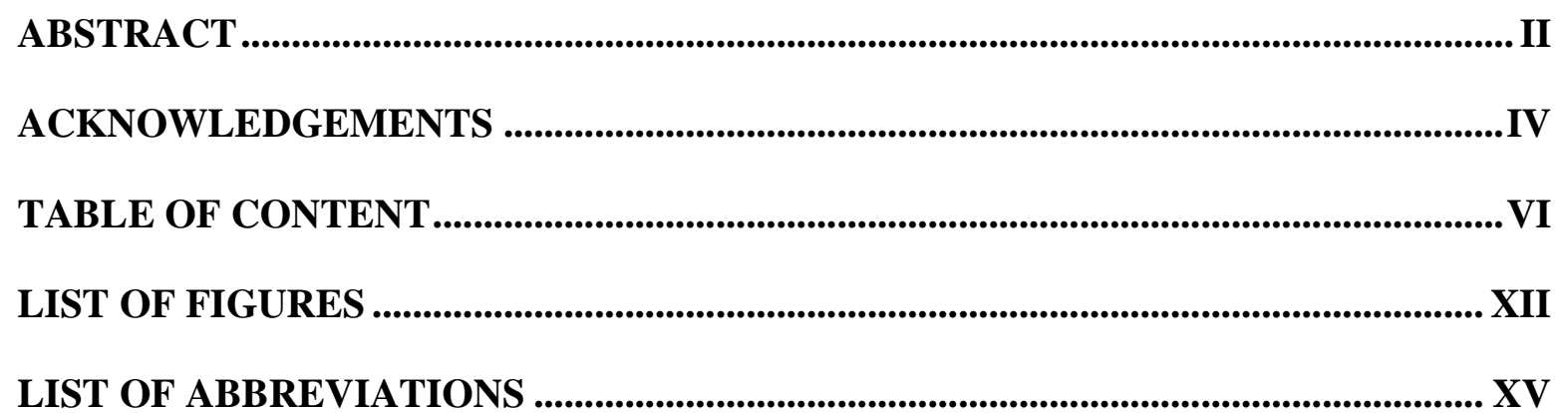

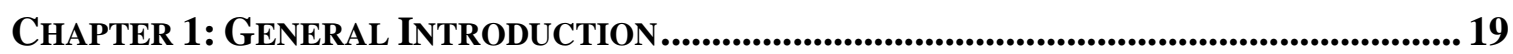

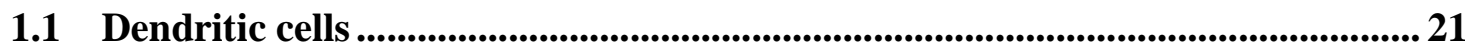

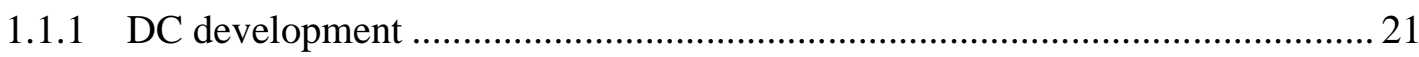

1.1.1.1 Development of DC from myeloid precursors......................................... 23

1.1.1.2 Development of DC from lymphoid precursors ...................................... 23

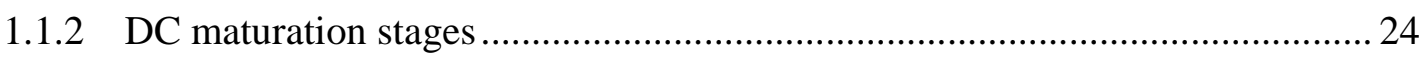

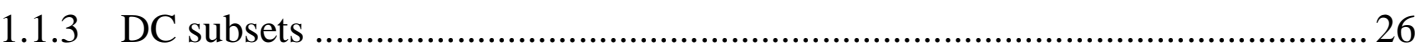

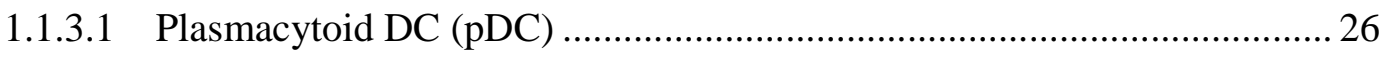

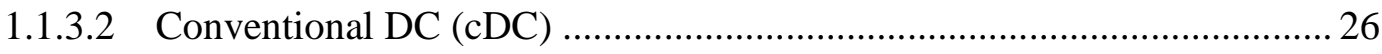

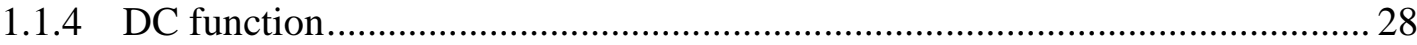

1.1.4.1 Migration of DC to secondary lymphoid organs …................................... 28

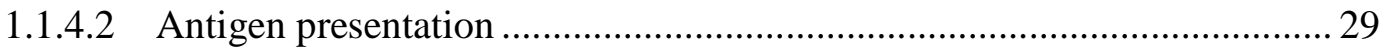

1.1.4.2.1 Major histocompatibility complex (MHC) expression .......................2 29

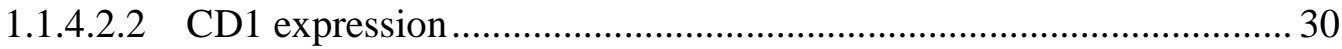

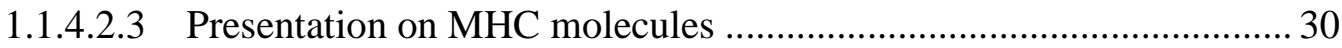

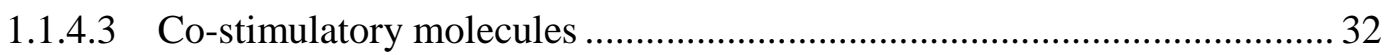

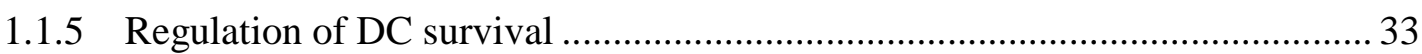

1.1.5.1 Mechanisms of DC survival and regulation............................................ 34

1.1.5.2 Effect of DC survival on T cell responses ............................................ 35

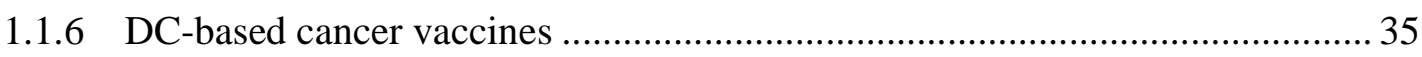

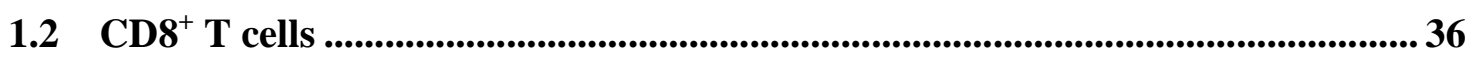

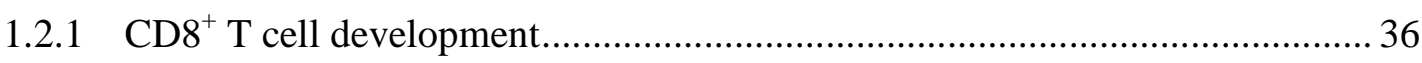

1.2.2 Activation and differentiation of naïve $\mathrm{CD}^{+} \mathrm{T}$ cells ....................................... 36 


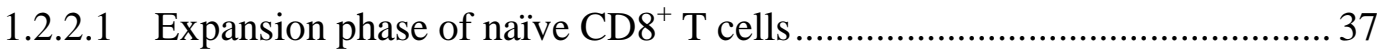

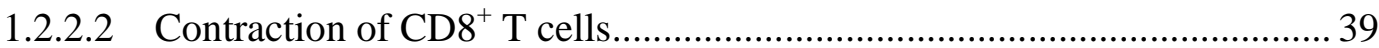

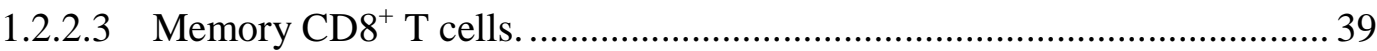

1.2.3 Effector functions of activated $\mathrm{CD}^{+} \mathrm{T}$ cells ................................................. 40

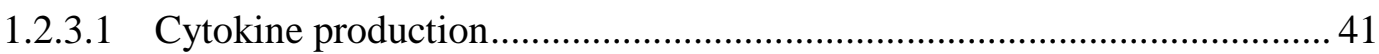

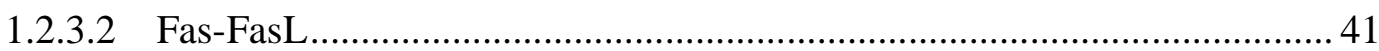

1.2.3.3 Perforin and the perforin/granzyme cytotoxic pathway........................... 41

1.2.3.4 Proposed models for perforin-mediated delivery of granzymes into target

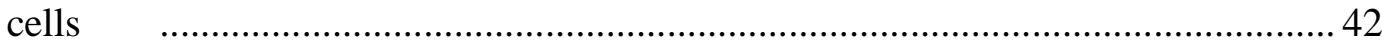

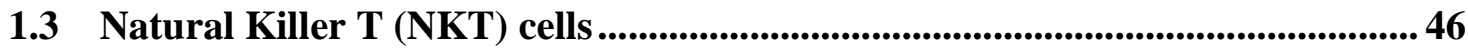

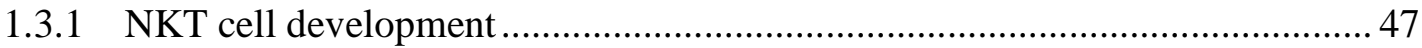

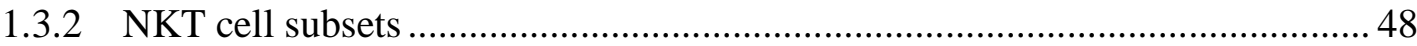

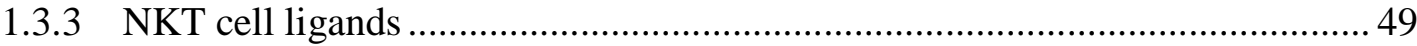

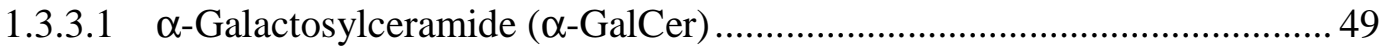

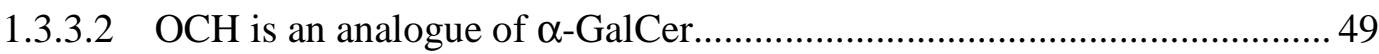

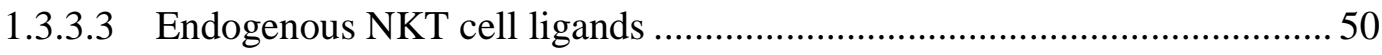

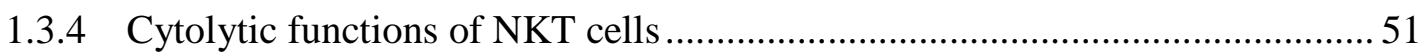

1.3.5 Immunoregulatory functions of NKT cells ................................................ 52

1.4 DC killing by $\mathrm{CD8}^{+} \mathrm{T}$ cells and NKT cells ..........................................................55

1.5 Aims...............................................................................................................................................5 57

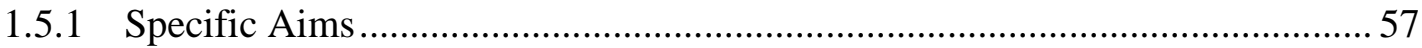

Chapter 2: MATERIALS ANd Methods ..........................................................................5 59

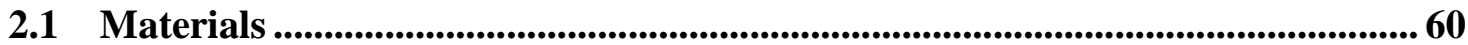

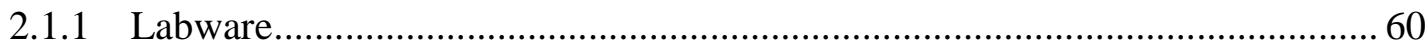

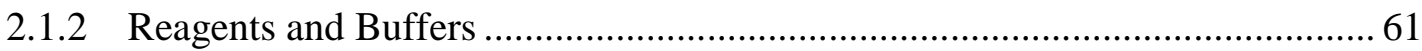

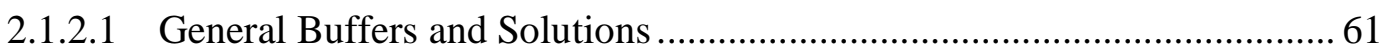

2.1.2.2 Solutions for enzyme linked immunosorbent assay (ELISA)...................65

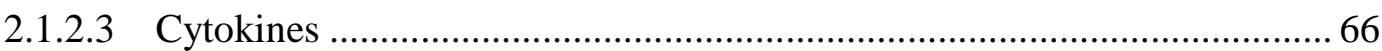

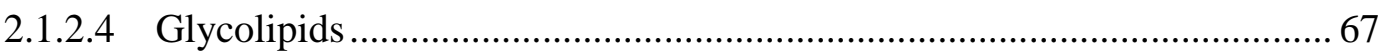

2.1.2.5 Antibodies, pentamers/multimers and secondary step reagents................. 68

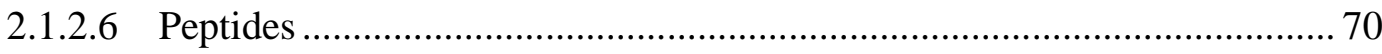




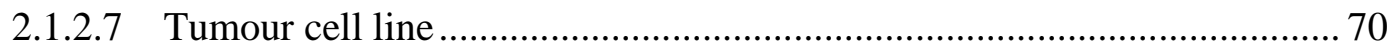

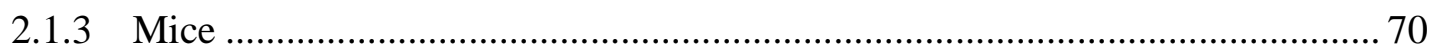

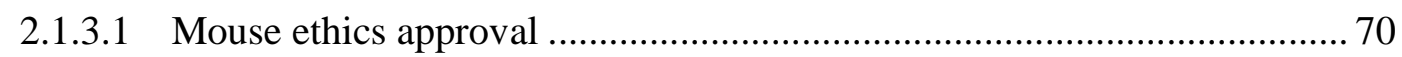

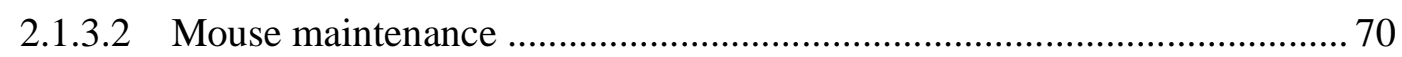

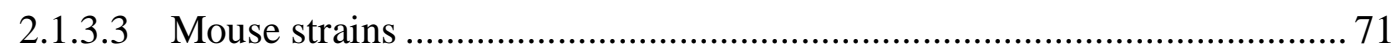

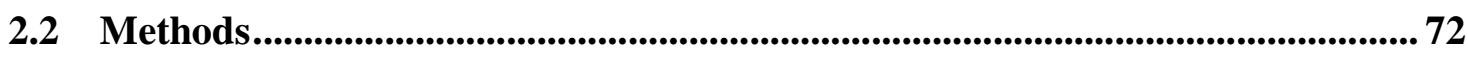

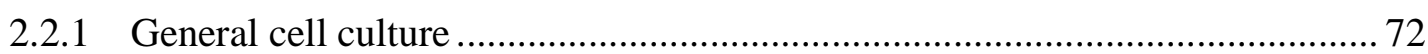

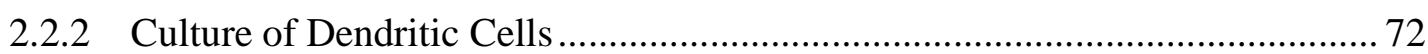

2.2.2.1 Culture from Bone marrow (BM) precursors ........................................... 72

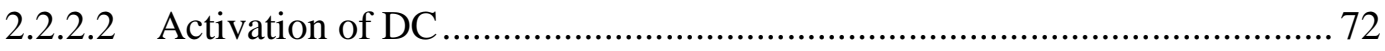

2.2.2.3 Loading of DC with $\alpha$-Galactosylceramide ( $\alpha$-GalCer)......................... 73

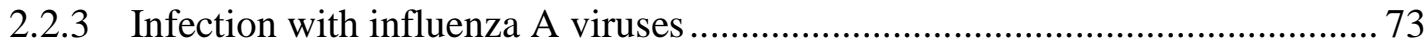

2.2.3.1 Preparation of influenza A virus stocks ................................................. 73

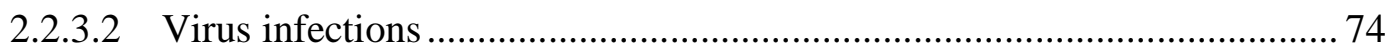

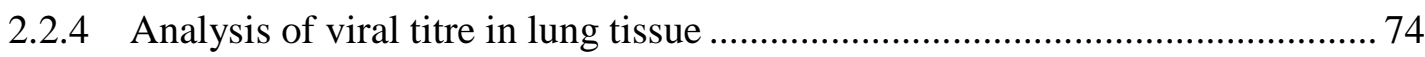

2.2.4.1 Collection of lung tissue ..................................................................... 74

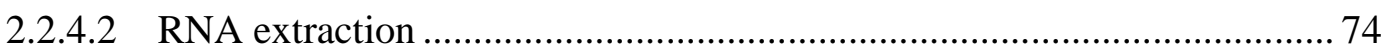

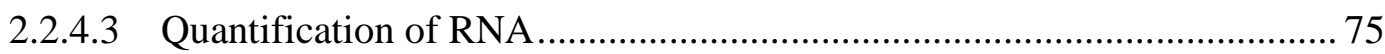

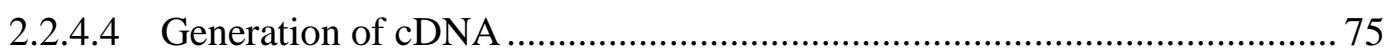

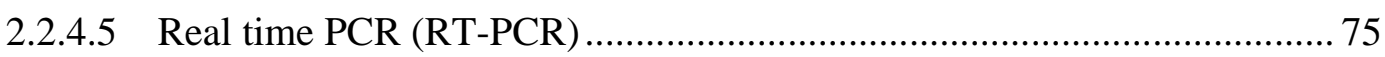

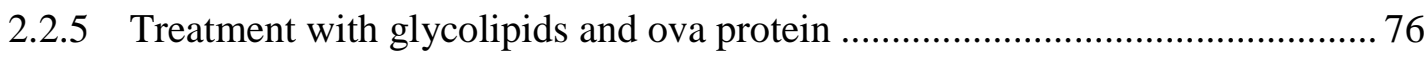

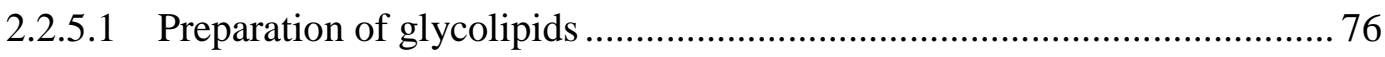

2.2.5.2 Preparation of chicken ovalbumin (ova) protein...................................... 76

2.2.5.3 Injection of glycolipids and ova protein ................................................. 77

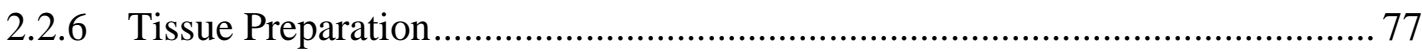

2.2.6.1 Bronchoalveolar lavage (BAL) ........................................................... 77

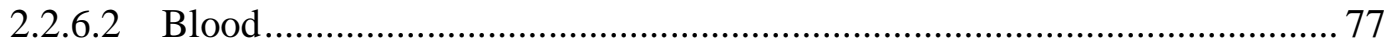

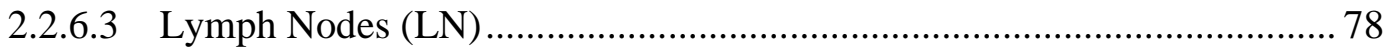

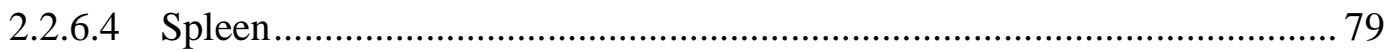

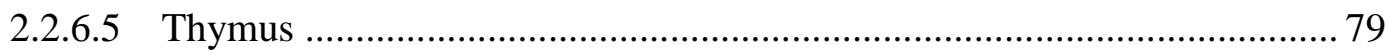

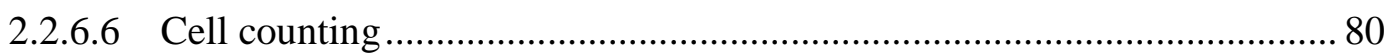

2.2.7 Fluorescent labelling of cells and analysis by flow cytometry ........................ 80

2.2.7.1 Labelling of proteins and antibodies ..................................................... 80 
2.2.7.2 Detection of cell surface marker expression 80

2.2.7.3 Detection of antigen specific TCR using MHC I pentamers 81

2.2.7.4 Loading of CD1d tetramer with $\alpha$-GalCer and detection of NKT cells with the CD1d tetramer 82

2.2.7.5 Detection of intracellular IFN- $\gamma$ after in vitro restimulation 82

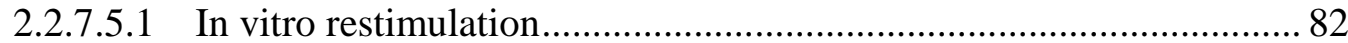

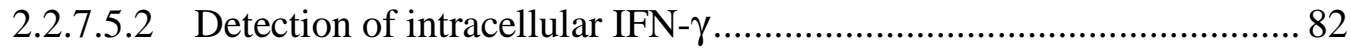

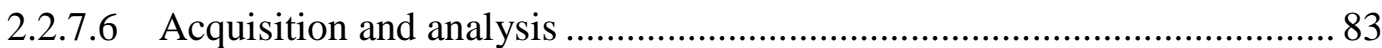

2.2.8 Cell purification by Magnetic Cell Separation (MACS) ................................. 83

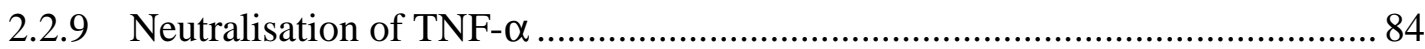

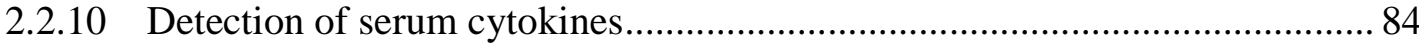

2.2.11 Detection of ova-specific antibodies by ELISA …..................................... 85

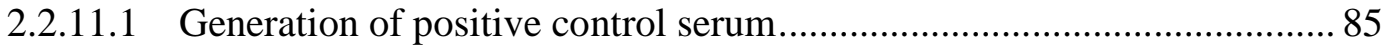

2.2.11.2 Ova-specific IgG1 ELISA of murine serum ....................................... 85

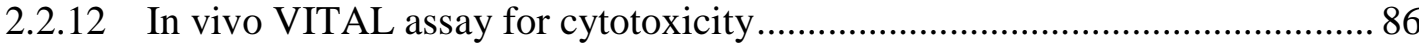

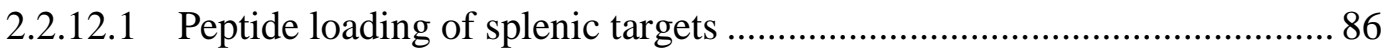

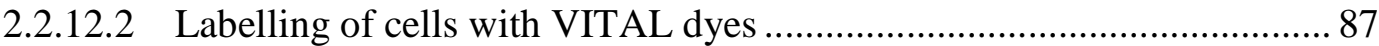

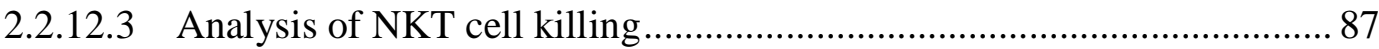

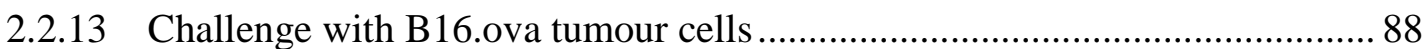

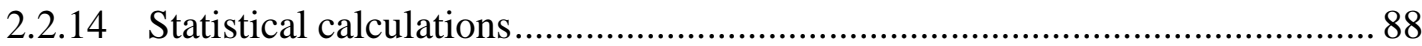

CHAPTER 3: ChARACTERISATION OF IMMUNE RESPONSES TO INFLUENZA A VIRUS IN PKO

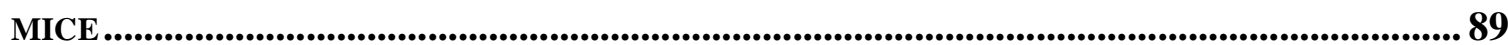

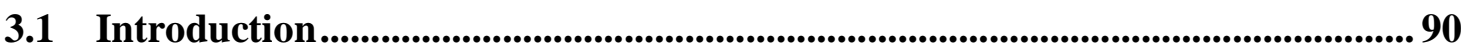

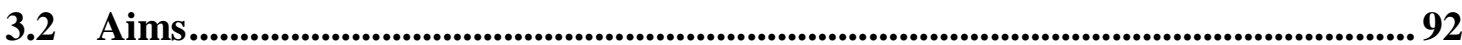

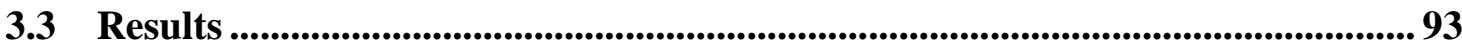

3.3.1 Influenza A virus establishes infections of comparable severity in C57BL/6 and

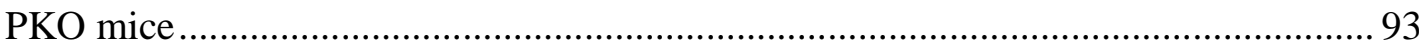

3.3.2 Measurement of viral load in the lung tissue after a primary influenza infection 97

3.3.3 Evaluation of the influenza-specific T cell response using MHC I pentamers in C57BL/6 and PKO mice during a primary infection .99 
3.3.4 Evaluation of the influenza-specific T cell response using MHC I pentamers in C57BL/6 and PKO mice during a secondary infection 105

3.3.5 Evaluation of the influenza-specific $\mathrm{T}$ cell response by measuring the production of specific IFN- $\gamma$ in C57BL/6 and PKO mice 109

3.3.6 Expression of KLRG1 by influenza-specific $\mathrm{CD}^{+} \mathrm{T}$ cells .......................... 118

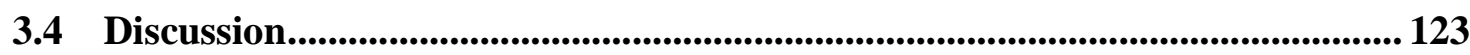

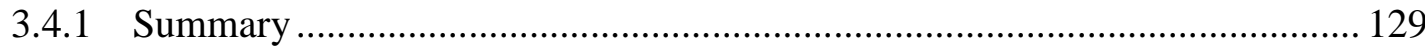

CHAPTER 4: DIFFERENTIAL SURVIVAL OF SPLENIC DC SUBSETS AFTER TREATMENT WITH

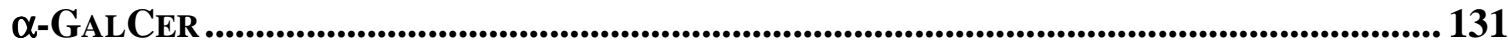

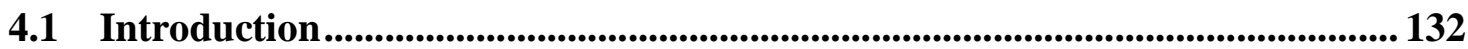

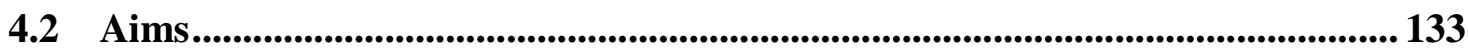

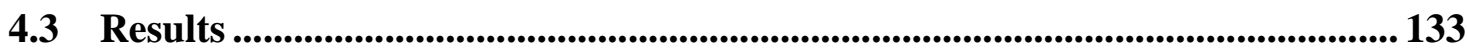

4.3.1 $\mathrm{CD}^{+} \mathrm{DC}$ are depleted by treatment with $\alpha$-GalCer and ova protein ............. 133

4.3.2 The decrease in the splenic $\mathrm{CD}^{+} \mathrm{DC}$ subset is not due to perforin-dependent

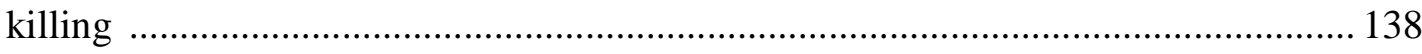

4.3.3 The decrease in $\mathrm{CD}^{+} \mathrm{DC}$ is not due to a Fas-dependent mechanism ............ 142

4.3.4 Assessing the ova-specific $\mathrm{CD}^{+} \mathrm{T}$ cell response after treatment with $\alpha-\mathrm{GalCer}$

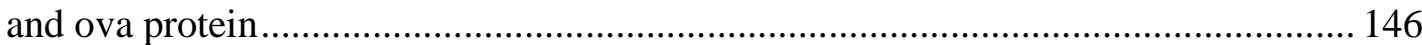

4.3.5 Assessing DC survival after a boost of ova protein ..................................... 149

4.3.6 IgG1 levels, and number of basophils are similar in PKO and C57BL/6 mice 152

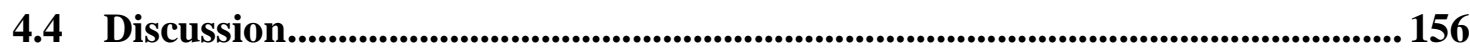

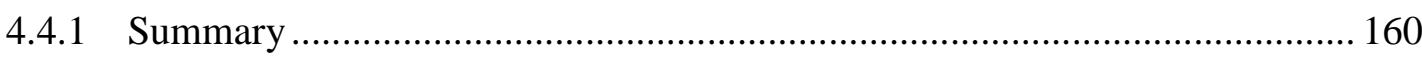

CHAPTER 5: STUDY OF THE MECHANISM OF $\mathrm{CD8}^{+}$DC LOSS AFTER TREATMENT WITH $\alpha$ -

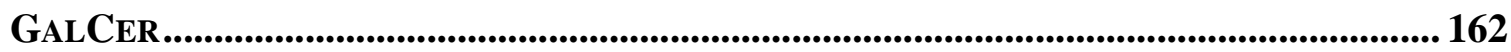

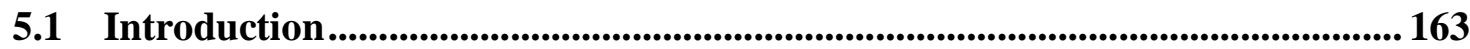

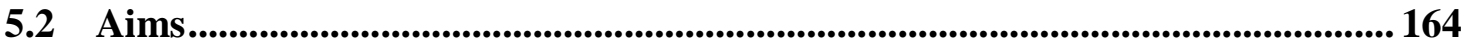

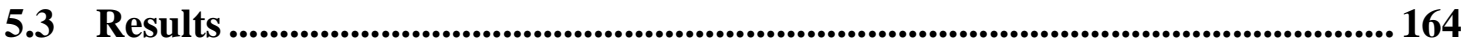

5.3.1 The decrease in $\mathrm{CD}^{+} \mathrm{DC}$ is not due to the down regulation of CD8 expression 
5.3.2 The decrease in $\mathrm{CD}^{+} \mathrm{DC}$ requires the presence of CD1d and/or NKT cells 172

5.3.3 The reduction in $\mathrm{CD}^{+} \mathrm{DC}$ after $\alpha$-GalCer treatment occurs in a dose

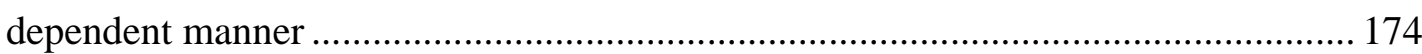

5.3.4 The decline in $\mathrm{CD}^{+} \mathrm{DC}$ after $\alpha$-GalCer treatment is transient ...................... 176

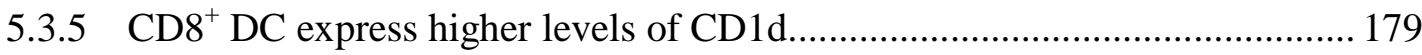

5.3.6 Treatment with the $\alpha-\mathrm{GalCer}$ analogue $\mathrm{OCH}$ induces a decrease in the number

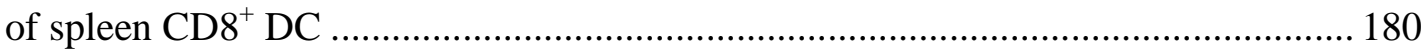

5.3.7 The decline in $\mathrm{CD}^{+} \mathrm{DC}$ may not be mediated through a direct interaction with NKT cells 182

5.3.8 IFN- $\gamma$ does not mediate the decrease in $\mathrm{CD}^{+} \mathrm{DC}$ after treatment with $\alpha$-GalCer 185

5.3.9 TNF- $\alpha$ plays a role in the reduction of $\mathrm{CD} 8^{+} \mathrm{DC}$ after $\alpha$-GalCer treatment.. 188 5.3.10 Reduced numbers of $\mathrm{CD} 8^{+} \mathrm{DC}$ after $\alpha$-GalCer treatment do not affect ovaspecific immune responses 191

5.3.11 Neutralisation of TNF- $\alpha$ leads to reduced NKT cell activation 199

5.4 Discussion 201

5.4.1 Summary 208

ChAPTER 6: GeNERAL DiscuSSION 209

6.1 Summary 216

6.2 Future Directions 218

REFERENCES 220 


\section{List of Figures}

Figure 1.1 DC Development from haematopoietic precursors ............................................ 22

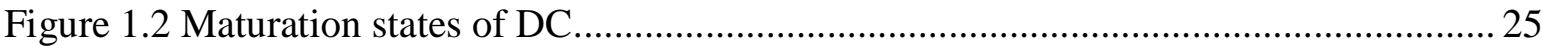

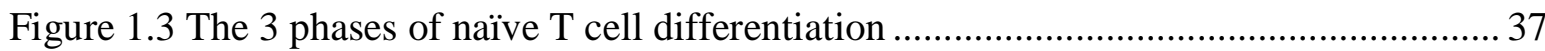

Figure 1.4 Models of perforin-dependent delivery of granzymes to the target cell.................. 44

Figure 1.5 Mouse NKT cells share some characteristics of NK and T cells .......................... 47

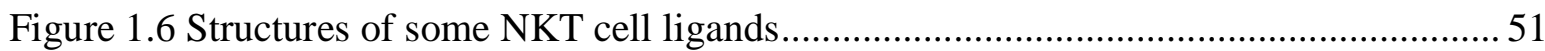

Figure 1.7 NKT cells interact with and activate many different cell types .............................5 54

Figure 3.1 Weight loss after primary and secondary influenza infection. .............................. 95

Figure 3.2 Clearance of the HKx31 virus from the lung tissue is slightly delayed in PKO mice

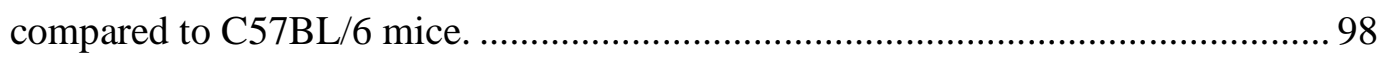

Figure 3.3 C57BL/6 and PKO mice generate similar influenza-specific $\mathrm{CD}^{+} \mathrm{T}$ cells responses

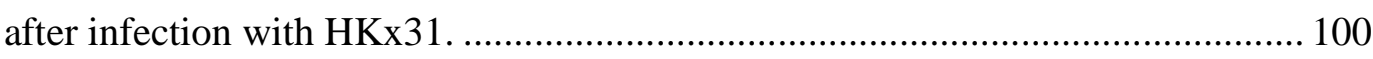

Figure 3.4 The number of $\mathrm{CD}^{+} \mathrm{D}^{\mathrm{b}}-\mathrm{NP}_{366-374}{ }^{+} \mathrm{T}$ cells found in MLN and spleen is comparable between C57BL/6 and PKO mice after a primary infection with $\mathrm{HKx} 31$. 102

Figure 3.5 Similar percentages of $\mathrm{CD}^{+} \mathrm{D}^{\mathrm{b}}-\mathrm{NP}_{366-374}{ }^{+} \mathrm{T}$ cells are found during an influenza

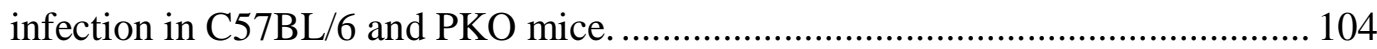

Figure 3.6 Detection of $\mathrm{D}^{\mathrm{b}}-\mathrm{NP}_{366-374}{ }^{+}$and $\mathrm{D}^{\mathrm{b}}-\mathrm{PA}_{224-233} \mathrm{CD} 8^{+} \mathrm{T}$ cells during a primary and a secondary influenza infection.

Figure 3.7 A reduced percentage of $\mathrm{CD}^{+} \mathrm{D}^{\mathrm{b}}-\mathrm{NP}_{366-374}{ }^{+} \mathrm{T}$ cells is observed in PKO mice compared to C57BL/6 mice during a secondary influenza infection.

Figure 3.8 Detection of intracellular IFN- $\gamma$ in $\mathrm{NP}_{366-374}{ }^{+}$responsive $\mathrm{T}$ cells in different tissues from C57BL/6 and PKO mice undergoing a primary influenza infection. 110

Figure 3.9 A similar number of influenza-specific T cells produce IFN- $\gamma$ over the course of a primary infection in C57BL/6 and PKO mice

Figure 3.10 The number of antigen-specific $\mathrm{CD} 8^{+} \mathrm{T}$ cells producing IFN- $\gamma$ in the MLN after a secondary influenza challenge.

Figure 3.11 The number of antigen-specific $\mathrm{CD}^{+} \mathrm{T}$ cells producing IFN- $\gamma$ in the spleen after a secondary influenza challenge.

Figure 3.12 Expression of KLRG1 on $\mathrm{CD}^{+} \mathrm{D}^{\mathrm{b}}-\mathrm{NP}_{366-374}{ }^{+}$blood $\mathrm{T}$ cells after a secondary influenza infection. 
Figure 3.13 The percentages of influenza-specific $\mathrm{CD} 8^{+} \mathrm{T}$ cells expressing KLRG1 in C57BL/6 and PKO mice are similar.

Figure 4.1 Treatment with $\alpha$-GalCer and ova protein does not affect the total number of splenic DC

Figure 4.2 Treatment with $\alpha$-GalCer induces a decrease in the number of splenic CD $8^{+} \mathrm{DC}$.

Figure 4.3 The decline in the $\mathrm{CD} 8^{+} \mathrm{DC}$ subset after $\alpha$-GalCer treatment occurs in a perforinindependent manner.

Figure 4.4 NKT cells can kill $\alpha$-GalCer pulsed targets in a perforin-independent manner.... 141

Figure 4.5 The number of NKT cells in the thymus and spleen of C57BL/6 and B6.lpr mice was comparable.

Figure 4.6 Fas deficiency has a minor effect on the loss of $C D 8^{+} \mathrm{DC}$ after $\alpha$-GalCer treatment.

Figure 4.7 C57BL/6 and PKO mice treated with $\alpha-G a l C e r$ and ova protein generate comparable ova-specific $\mathrm{CD} 8^{+} \mathrm{T}$ cell responses

Figure $4.8 \mathrm{~A}$ boost of ova protein did not induce an increased percentage of $\mathrm{CD} 8^{+} \mathrm{K}^{\mathrm{b}}$-ova 257 $264^{+} \mathrm{T}$ cells

Figure 4.9 Boosting with ova protein does not lead to a change in the number of spleen DC.

Figure 4.10 The levels of ova-specific IgG1 in the serum of C57BL/6 and PKO mice were similar

Figure 4.11 C57BL/6 and PKO mice show a similar percentage of basophils present in the peripheral blood after treatment with $\alpha$-GalCer and ova protein

Figure 5.1 $\mathrm{EGFP}^{+}$splenic DC from the Langerin-EGFP mice are phenotypically similar to splenic $\mathrm{CD}^{+}$DC from C57BL/6 mice.

Figure 5.2 The number of splenic DC expressing Langerin is reduced after treatment with $\alpha$ -

GalCer.

Figure 5.3 The number of $\mathrm{CD} 11 \mathrm{c}^{+} \mathrm{CD} 24^{\mathrm{hi}}$ cells declines after $\alpha$-GalCer treatment. 168

Figure 5.4 DEC 205 is a marker of steady state but not activated splenic CD8 ${ }^{+}$DC

Figure 5.5 The decrease in $\mathrm{CD}^{+} \mathrm{DC}$ after $\alpha$-GalCer treatment requires NKT cells

Figure 5.6 The decrease in the number of $\mathrm{CD} 8^{+} \mathrm{DC}$ occurs in a dose dependent manner.... 175

Figure 5.7 The loss of $\mathrm{CD}^{+} \mathrm{DC}$ after $\alpha$-GalCer treatment is transient.

Figure 5.8 CD8 ${ }^{+}$DC express higher levels of surface CD1d than CD8 ${ }^{-}$DC. 
Figure 5.9 Treatment with the $\alpha$-GalCer analogue $\mathrm{OCH}$ induces a decline in the number of $\mathrm{CD} 8^{+} \mathrm{DC}$.

Figure 5.10 The decrease in the number of $\mathrm{CD} 8^{+} \mathrm{DC}$ may not require a direct interaction with NKT cells.

Figure 5.11 IFN- $\gamma$ does not mediate the loss of $\mathrm{CD}^{+} \mathrm{DC}$ after $\alpha$-GalCer treatment. 186

Figure 5.12 TNF- $\alpha$ plays a role in the decrease of $\mathrm{CD} 8^{+} \mathrm{DC}$ after $\alpha-\mathrm{GalCer}$ treatment. 189

Figure 5.13 Treatment with a TNF- $\alpha$ neutralising antibody reduced the upregulation of CD86 on DC after $\alpha$-GalCer treatment.

Figure 5.14 The lower dose of $\alpha$-GalCer only affects the early ova-specific $\mathrm{CD} 8^{+} \mathrm{T}$ cell response.

Figure 5.15 Administration of ova together with low or high doses of $\alpha$-GalCer elicits similar tumour protection. 195

Figure 5.16 Blocking TNF- $\alpha$ during treatment with $\alpha$-GalCer and ova improves the early but not the late $\mathrm{CD}^{+} \mathrm{T}$ cell response. 197

Figure 5.17 Treatment with anti-TNF- $\alpha$ leads to reduced serum cytokine levels after $\alpha$-GalCer treatment 200

Figure 5.18 A schematic diagram of the mechanisms of $\alpha$-GalCer presentation after adoptive transfer of BMDC loaded with $\alpha$-GalCer. 


\section{List of Abbreviations}

$\alpha$-GalCer Alpha-Galactosyleramide

ABTS 2,2'-azino-bis(3-ethylbenz-thiazoline6-solfonic acid)

ACT Ammonium Chloride Tris

APC Antigen presenting cell

APCy Allophycocyanin

B16.ova Ova transfected B16 melanoma cell line

BAL Bronchoalveolar lavage

BM Bone marrow

BMDC Bone marrow dendritic cells

BrdU Bromodeoxyuridine

BSA Bovine Serum Albumin

CD Cluster of differentiation

cDC Conventional dendritic cell

cDNA Complementary DNA

CFSE Carboxyfluorescein diacetate succinimidyl ester

cIMDM Complete Iscove's Modified Dulbecco's Medium

CLP Common lymphoid precursor

CMP Common myeloid precursor

CTL Cytotoxic T Lymphocyte

CTLA-4 Cytotoxic T lymphocyte associated antigen 4

CTO Cell Tracker Orange

DAPI 4',6-diamidino-2-phenylindole

DC Dendritic cell

DMSO Dimethyl Sulfoxide

DN Double negative

DNA Deoxyribonucleic acid

DP Double positive

EDTA Ethylenediaminetetraacetic Acid

EGFP Enhnaced Green Fluorescent Protein

ELISA Enzyme Linked Immunosorbent Assay 
FACS Fluorescence Activated Cell Sorting

Fc $\gamma \mathbf{R} \quad \mathrm{Fc} \gamma$ receptor

FCS Foetal Calf Serum

FHL Familial Hemophagocytic Lymphohistiocytosis

FITC Fluorescein isothiocyanate

Flt-3 FMS-like tyrosine kinase 3

FSC Forward scatter

GM-CSF Granulocyte macrophage colony stimulating factor

GP 33-41 Glycoprotein fragment 33-41 from LCMV

HAU Hemagglutinating Unit

HEV High Endothelial Venule

HSC Hemopoietic stem cells

IFN- $\boldsymbol{\gamma}$ Interferon $\boldsymbol{\gamma}$

IgE Immunoglobulin E

IgG Immunoglobulin G

IgG1 Immunoglobulin G subclass 1

IgM Immunoglobulin M

IL Interleukin

IMDM Iscoves modified Dulbecco's medium

i.n. Intranasally

i.p. Intraperitoneally

i.v. Intravenously

KLRG1 Killer cell lectin-like receptor G 1

KO Knockout

L Ligand

LCMV Lymphocytic Choriomeningitis virus

LN Lymph node

LPS Lipopolysaccharide

mAb Monoclonal antibody

MACS Magnetic Cell Sorting

MFI Mean Fluorescence Intensity

MHC Major histocompatibility complex 


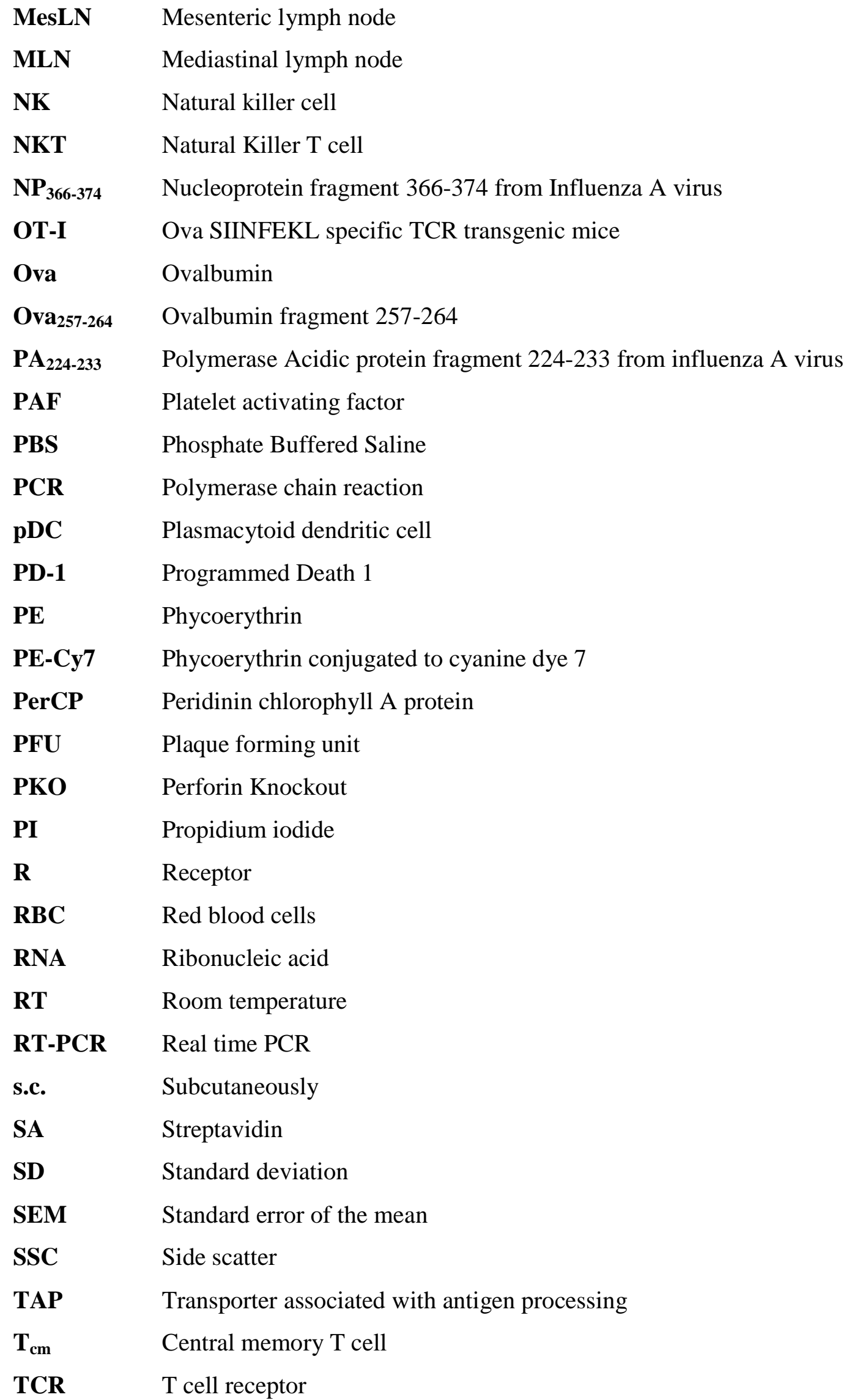


TGF- $\beta$ Transforming growth factor $\beta$

$\mathbf{T}_{\text {em }} \quad$ Effector memory $\mathrm{T}$ cell

Th Thelper cell

TLRs Toll-like receptors

TNF- $\alpha$ Tumor necrosis factor $\alpha$

TNFR TNF receptor

TRAIL TNF related apoptosis-inducing ligand

TRANCE TNF related activation-induced cytokine

$\mathbf{V} \boldsymbol{\alpha} \quad$ Variable $\alpha$ region of the T cell receptor

$\mathbf{V} \boldsymbol{\beta} \quad$ Variable $\beta$ region of the T cell receptor

VSV Vesicular stomatitis virus

WT Wild-type 


\section{Chapter 1:}

General Introduction 
The main role of the immune system is to protect the host from foreign pathogens, including viral, bacterial, fungal and parasitic infections. Two branches of the immune system exist: innate and adaptive immunity. The innate immune system provides the first line of defense against environmental organisms and is essential for the control of common infections. Neutrophils and macrophages eliminate invading pathogens by phagocytosis, while natural killer (NK) cells directly kill infected cells. In addition, natural killer T (NKT) cells are rapidly activated upon recognition of bacterial lipids and enhance immune responses. Dendritic cells (DC) take up foreign antigen and present parts of these antigens on their cell surface for recognition by other cells of the immune system. In contrast, the adaptive immune response is activated in response to specific antigens and employs antigen specific $\mathrm{T}$ and $\mathrm{B}$ lymphocytes for efficient pathogen elimination. Furthermore, the adaptive immune response also provides protection against subsequent re-infection with the same pathogen, a phenomenon known as immunological memory.

DC play a central role in immune responses because they interact with cells from both the innate and adaptive branches of the immune system. DC can directly encounter pathogens that induce the secretion of cytokines, which in turn can activate cells of the innate immune system, such as neutrophils, macrophages, NK and NKT cells, ultimately resulting in pathogen clearance. In addition, DC can directly interact with T and NKT cells resulting in their activation, leading to the eradication of the invading pathogens. DC-NKT cell interactions also result in the activation of NK cells, T and B cells, further amplifying the immune response. Thus, through several different interactions, DC provide an important link between innate and adaptive immunity leading to the generation of an effective immune response.

Since DC are important in eliciting effective T cell responses, which are necessary for antitumour immunity, DC presenting tumour antigens have been used as an anti-cancer immunotherapeutic treatment. However, after multiple clinical trials only a small number of patients have benefited from DC treatment. The survival and regulation of DC is vital for generating an effective $\mathrm{T}$ cell response, and hence an effective anti-cancer treatment. Thus, the overall goal of this thesis was to evaluate DC survival in two different in vivo models and assess whether $\mathrm{CD} 8^{+} \mathrm{T}$ cells and NKT cells, which both have cytolytic potential, eliminate DC through the use of perforin, a molecule important for target cell 
lysis. By gaining a better understanding of the regulation of DC survival, it will be possible to generate more effective anti-cancer treatments.

This introductory chapter summaries the functions of DC and their importance in activating naïve $T$ cells. It also reviews the current literature addressing the regulation of DC survival and outlines the effect this has on $\mathrm{T}$ cell responses. In addition, as this thesis focuses on the role of $\mathrm{CD}^{+} \mathrm{T}$ cells and NKT cells in DC elimination, an overview of these cells and their ability to eliminate DC is presented.

\subsection{Dendritic cells}

DC are sentinels of the immune system (1); they are highly efficient antigen presenting cells (APCs) and play a central role in the induction and regulation of most adaptive immune responses. DC reside in most peripheral tissues in an 'immature' state where they are continuously sampling the antigenic environment. In the steady state, DC spontaneously 'mature' and constitutively migrate to the lymph nodes (LN) where they present self-antigen to T cells. In the absence of pro-inflammatory cytokines this presentation results in peripheral tolerance. However, upon encountering microbial products DC become 'activated' and present the captured antigen to T cells in the presence of pro-inflammatory cytokines, resulting in T cell activation, and culminating in an immune response.

DC are a heterogenous cell population and are comprised of two main subpopulations: plasmacytoid DC (pDC) and so called "conventional” DC (cDC). Based on phenotypic markers and localisation, these two main categories can be further subdivided into functionally distinct subsets. These DC subsets will be discussed further in section 1.1.3.

\subsubsection{DC development}

DC are continuously produced from bone marrow (BM) hemopoietic stem cells (HSC) (2). All HSC-derived cells develop along either the lymphoid or myeloid pathway, through a committed but lineage restricted common lymphoid precursor (CLP) or common myeloid precursor (CMP) $((3,4)$, Figure 1.1). Studies of DC development provide evidence that 
DC subsets can originate through both myeloid and lymphoid pathways, from a CMP or CLP (5-7). FMS-related tyrosine kinase 3 (Flt3), a member of the class III receptor tyrosine kinase family, is expressed on early progenitor cells from the BM (8). Both CMP and CLP are heterogeneous for Flt3 expression, with most CLP expressing Flt3, whereas only a minor fraction of CMP expresses Flt3 $(3,9,10)$. Flt3 ligand (Flt3L) has been shown to act as a growth factor for hemopoietic progenitors $(8,11,12)$. Early DC precursors are found only among the Flt $3^{+}$fractions of both CMP and CLP subpopulations $(9,10)$, demonstrating that DC can be of either myeloid or lymphoid origin (Figure 1.1).

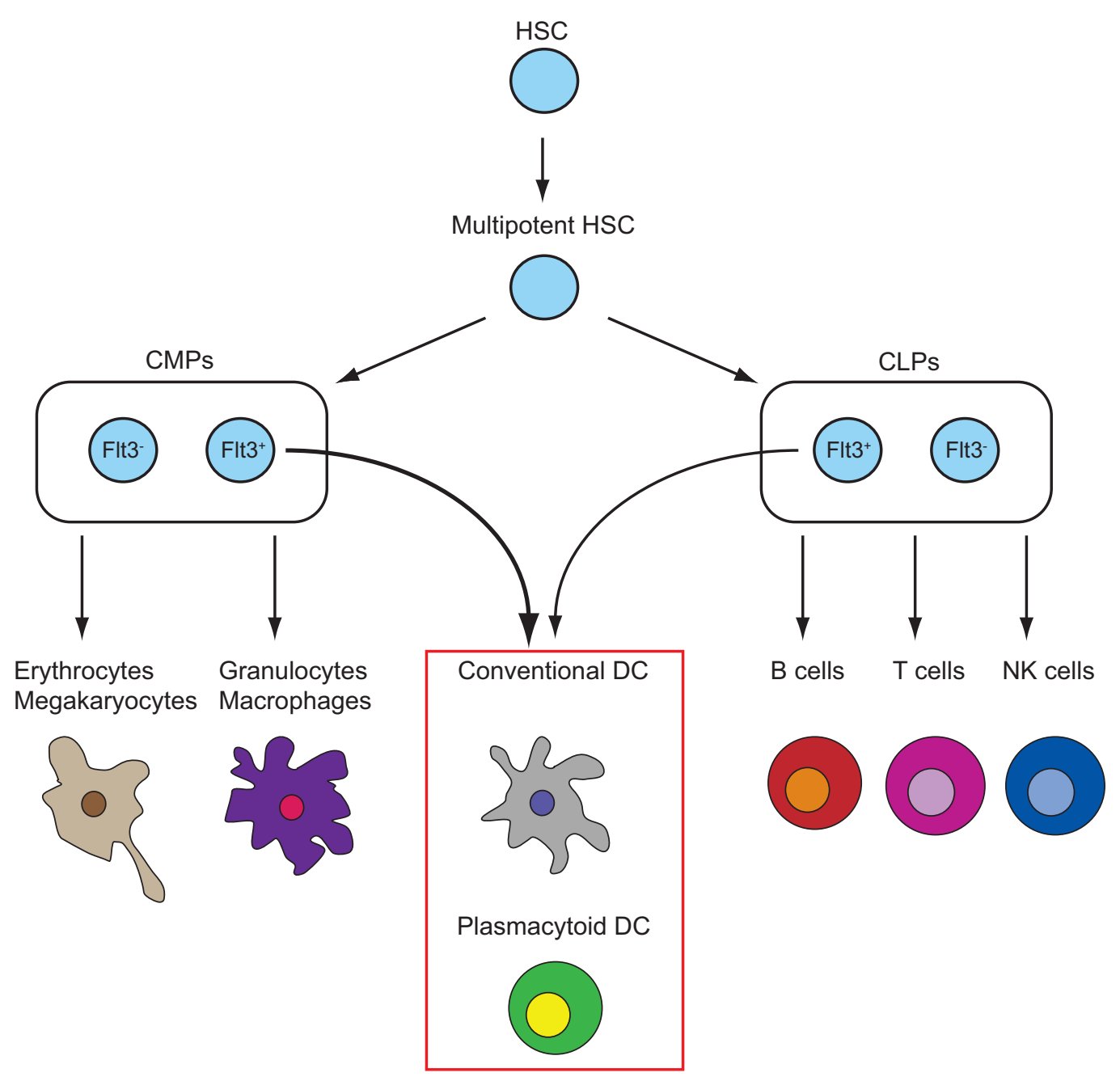

\section{Figure 1.1 DC Development from haematopoietic precursors}

Both conventional and plasmacytoid DC can be generated from the Flt $3^{+}$expressing early myeloid and lymphoid progenitors. HSC: Hemopoietic stem cell, CMPs: Common myeloid progenitors and CLPs: Common lymphoid progenitors. Adapted from Shortman and Naik (13). 


\subsubsection{Development of DC from myeloid precursors}

DC were originally considered to be of myeloid origin because early studies demonstrated that cultured mouse BM myeloid precursors had the capacity to produce macrophages, granulocytes and DC in the presence of granulocyte macrophage colony stimulating factor (GM-CSF, (14)). Similar results were seen in studies of human cells (15-17). Direct evidence for a myeloid origin of DC was obtained when transplantation of mouse CMPs into irradiated recipients led to the reconstitution of all the major DC populations found in mouse lymphoid tissues $(6,7,18)$. Further studies demonstrated that the capability of CMPs to produce all $\mathrm{cDC}$ and $\mathrm{pDC}$ subsets found in the mouse spleen and thymus was restricted to the Flt $3^{+}$fraction ((9), Figure 1.1).

\subsubsection{Development of DC from lymphoid precursors}

The first suggestion of a possible lymphoid origin of DC came when mouse thymic DC and a subset of splenic DC were found to express a number of cell surface markers normally expressed on lymphoid cells (19). However, the first direct evidence was obtained when transfer of thymic lymphoid precursors into irradiated mice gave rise to both T cells and $\mathrm{CD}^{+}$thymic cDC (20). Recent studies have shown that BM CLPs can generate all DC populations identified in lymphoid tissues (Figure 1.1), but with a strong bias towards the development of the $\mathrm{CD} 8^{+} \mathrm{DC}$ subset $(5,7)$.

Based on the finding that pDC express many lymphoid markers, some authors have suggested that pDC may be exclusively of lymphoid origin. Human thymic pDCs were found to express the pre-T cell receptor $\alpha(\mathrm{pT} \alpha)(21,22)$. In addition, murine pDC were found to carry a rearranged immunoglobulin heavy chain $(\operatorname{IgH})$ D-J and express the Rag gene but lack TCR $\beta$ gene D-J rearrangements $(23,24)$. Further support for a lymphoid origin came from a study where over-expression of the dominant-negative transcription factors Id 2 or Id 3 in human hematopoietic progenitor cells blocked development of pDC, T cells and B cells but not myeloid DC (25). However, later studies have revealed that Flt $3^{+}$ cells within either CLPs or CMPs can differentiate into both $\mathrm{cDC}$ and $\mathrm{pDC}$ in culture and in vivo $((9,24,26)$, Figure 1.1). In support of this, $\mathrm{pDC}$ derived from CMPs were shown to carry D-J rearrangement of IgH genes (24). Thus, recent studies have established that pDC can arise from both myeloid and lymphoid precursors. 


\subsubsection{DC maturation stages}

In the absence of inflammation (steady state), DC continually patrol peripheral tissues in an immature state, poised to capture and process antigen. The immature state is characterised by low surface expression of major histocompatibility complex II (MHC II) molecules and co-stimulatory molecules along with a high capacity to capture antigen ((1), Figure 1.2). Immature DC can capture antigen by receptor mediated endocytosis through DEC 205 (CD205) (27) or the mannose receptor, CD206 (28). DC have also been reported to take up antigen by phagocytosis (29), pinocytosis (28) and they can take up immune complexes, consisting of antigens bound to $\mathrm{IgG}$, though immunoglobulin Fc $\gamma$ receptors (Fc $\gamma \mathrm{R})(30)$. The large array of antigen capture mechanisms partly accounts for DC being the most efficient APCs in the immune system.

Steady state immature DC can spontaneously mature, upregulating expression of MHC and co-stimulatory molecules along with lymph node (LN) homing receptors, thus allowing migration to the LN (31). Mature DC migrate in small numbers to the $\mathrm{LN}$, where they present self antigens to resting $\mathrm{CD}^{+} \mathrm{T}$ cells, leading to the induction of tolerance ((32-34), Figure 1.2). Directly targeting steady state DC with a monoclonal antibody (mAb) directed towards DEC 205 can cause tolerance in peripheral $\mathrm{CD}^{+}$and $\mathrm{CD}^{+} \mathrm{T}$ cells, by inducing $\mathrm{T}$ cell deletion or anergy $(35,36)$. Peripheral tolerance is thought to be responsible for elimination of auto-reactive T cells that have escaped thymic deletion (37). Thus, DC are also important for protecting the body from autoimmune diseases, by the induction of tolerance.

Activation of immature DC into a mature phenotype also occurs in response to microbial and viral products, such as lipopolysaccharide (LPS) and double stranded RNA, these DC will be termed "activated" DC. DC recognise these products via signalling through Tolllike receptors (TLRs) or other pattern recognition receptors (PRRs) $(38,39)$. Upon sensing these signals from pathogens, DC undergo a number of phenotypic changes, such as upregulation of expression of MHC molecules and the co-stimulatory molecules CD80, CD86 and CD40 ((1, 40), Figure 1.2). They also down regulate the expression of receptors involved in antigen uptake. Changes in the expression of chemokine receptors such as CCR7 and CXCR4 allow migration of DC from the peripheral tissues towards the T cell areas of the LN and spleen (41). Similar phenotypic changes are also observed in tolerogenic DC making it hard to distinguish between these two DC states. However, one 
important functional difference is the secretion of IL-12 and other cytokines by activated DC $(1,42-44)$, which is necessary for differentiation of $\mathrm{CD}^{+} \mathrm{T}$ cells into effector cells and the subsequent initiation of cell mediated immunity. Thus, changes in phenotype and cytokine secretion allow activated DC to become efficient APCs and initiators of $\mathrm{CD}^{+} \mathrm{T}$ cell mediated immunity $(31,45)$.

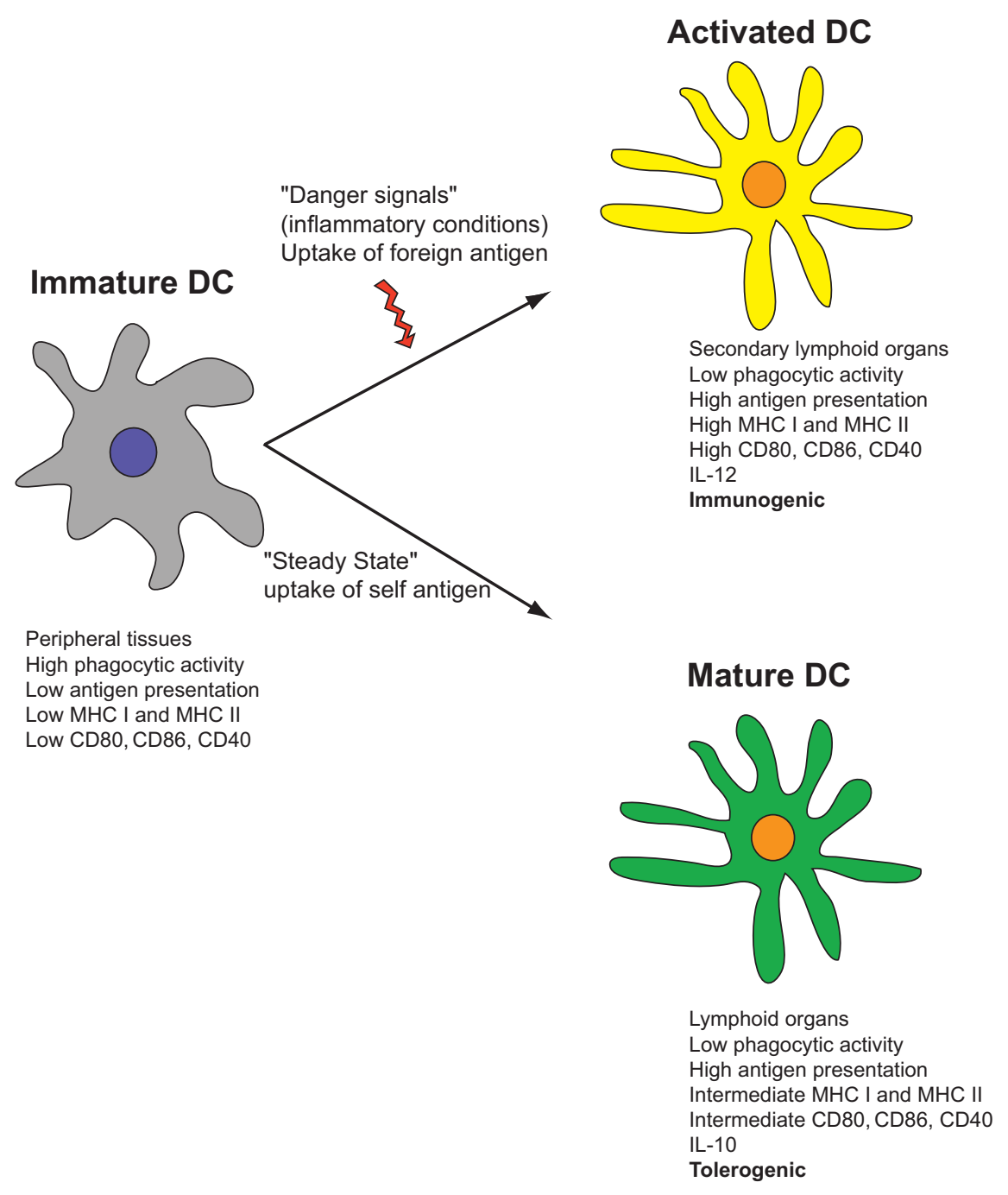

\section{Figure 1.2 Maturation states of DC}

Immature DC patrol the peripheral tissues where they have high endocytic activity. In the steady state (in the absence of inflammation) DC can mature and migrate to the LN where they present self-antigens and induce T cells anergy or tolerance. In the presence of 'danger signals' such as microbial products, DC become activated and upregulate expression of co-stimulatory molecules, inducing naïve $\mathrm{T}$ cell activation. 


\subsubsection{DC subsets}

There are many distinct DC subsets, each with a particular location and specialized function in the immune system (46). The two main types of DC, pDC and cDC, can be further subdivided, based on their location and the expression of a number of phenotypic markers. The different DC subsets are discussed in detail below.

\subsubsection{Plasmacytoid DC (pDC)}

Human pDC are identified as being $\mathrm{CD}^{+} \mathrm{CD}^{2} 5 \mathrm{RA}^{+} \mathrm{IL}_{-} 3 \mathrm{R}^{+} \mathrm{ILT}^{+} \mathrm{ILt1}^{-} \mathrm{CD} 11 \mathrm{c}$ lineage $^{-}$ cells, whereas mouse pDC are identified as $\mathrm{B} 220^{+} 1 \mathrm{y} 6 \mathrm{C}^{+} \mathrm{CD} 11 \mathrm{c}^{\text {lo }}$. $\mathrm{pDC}$ circulate through the blood and lymphoid tissues and only acquire the typical DC morphology after activation $(47,48)$. pDC become activated after recognising viral RNA and DNA via signalling through TLR7 and TLR9 (49). Following activation, pDC release large quantities of type I interferons (IFNs), which inhibit viral replication and activate NK cells, macrophages and cDC. Activation of $\mathrm{cDC}$ leads to increased antigen presentation to $\mathrm{T}$ cells resulting in more effective viral clearance. Once activated, pDC can also present antigens and induce $\mathrm{T}$ cell expansion, although not as efficiently as cDC. This is thought to be due to the reduced ability of pDC to endocytose antigens as well as their lower cell surface expression of MHC II molecules and co-stimulatory molecules (50). Thus, pDC play an important role in many immune responses, particularly antiviral responses and provide a link between innate and adaptive immunity $(47,48)$.

\subsubsection{Conventional DC (cDC)}

Conventional DC can be either migratory or reside in lymphoid tissue. The migratory cDC can be grouped based on the peripheral tissue of origin. Langerhans cells (LC) of the epidermis are the model migratory DC. They patrol the peripheral tissue and, upon recognition of microbial products differentiate into activated LC, migrating to the skin draining LN and presenting captured antigen. Until recently, LC were thought to differ from dermally derived DC, which also migrate to skin draining LN, by expression of Langerin $(51,52)$. However, recent data indicate that a large proportion of Langerin ${ }^{+}$cells in the $\mathrm{LN}$ originate from the dermis (53-55). These dermal Langerin ${ }^{+} \mathrm{DC}$ are indistinguishable from LC based on the expression levels of MHC II, CD11c and Langerin, but can be separated based on CD103 and CD11b expression $(53,56)$. LC and 
dermal DC also differ in their localisation within the LN, with LC primarily found in the T cell zone and dermal DC present near the B cell follicles (57). This distinct localisation may allow these different DC subsets to carry out non-overlapping roles in immune responses.

Lymphoid tissue-resident DC can be further subdivided based on their cell surface expression profile. The T cell markers, CD4 and CD8, are expressed on mouse DC (58) and are useful for segregating subsets. CD8 on DC is expressed as a CD8 $\alpha \alpha$ homodimer, unlike the CD8 $\alpha \beta$ heterodimer found on T cells (19). To date, there is no evidence that either CD4 or CD8 markers are functionally significant on mouse DC (46). Three DC subsets have been described in the spleen of mice: the $\mathrm{CD} 4^{-} \mathrm{CD} 8^{+}\left(\mathrm{CD} 8^{+} \mathrm{DC}\right)$, the $\mathrm{CD} 4^{+}$ $\mathrm{CD}^{-}\left(\mathrm{CD}^{-} \mathrm{DC}\right)$ and the $\mathrm{CD}^{-} \mathrm{CD}^{-}$(double negative [DN] DC) (58). Two additional markers used to segregate mouse DC subsets are CD11b (the integrin $\alpha_{M}$ chain of Mac-1) and c-type lectin DEC 205 (59). DEC 205 is expressed on CD8 ${ }^{+}$DC, whereas CD11b is present on CD8 ${ }^{-}$DC and DN DC (60). Thus, expression of surface markers distinguishes clearly between different DC subsets.

The $\mathrm{CD}^{+}$and $\mathrm{CD}^{-}$DC subsets in the spleen differ in immune functions and location. $\mathrm{CD}^{+} \mathrm{DC}$ are concentrated in the $\mathrm{T}$ cell areas and $\mathrm{CD}^{-} \mathrm{DC}$ are located in the marginal zones, however, the CD8 ${ }^{-}$DC migrate into the T cell zones upon stimulation with microbial products $(44,61,62)$. DC subsets also differ in function, with $\mathrm{CD}^{+} \mathrm{DC}$ producing the largest amount of IL-12 after stimulation $(44,63)$. DC subsets also differ in their ability to present antigen on MHC class I molecules (60), which will be discussed in more detail in section 1.1.4.2.3. These differences in immune functions correlate with a variant gene profile based on a global gene analysis by Affymetrix microarray (64). Taken together, these results show that the distinct lymphoid tissue resident DC may play diverse roles in immune responses.

Sensing invading pathogens is essential to induce an adaptive immune response in the host. DC express multiple TLRs which enables them to detect a range of microbial derived products. Studies of DC subsets isolated from humans and mice have reported a distinct repertoire of TLR. Freshly isolated human pDC express TLR7 and TLR9, whereas human cDC express TLR1, TLR2, TLR3, TLR5, TLR6 and TLR8, with the exception of human LC which do not express TLR3 (49). In mice, all splenic DC subsets express TLRs 1, 2, 4, 
6, 8 and 9 (65). However, TLR3 is only expressed by the splenic CD8 ${ }^{+}$and DN DC (65, 66). High expression of TLR 3 on $\mathrm{CD}^{+} \mathrm{DC}$ has been linked to their ability to present exogenous antigens on MHC I molecules (66). Moreover, mouse CD8 ${ }^{+}$DC lack TLR5 and TLR7 expression and fail to respond to TLR7 agonists $(65,67)$. Distinct TLR expression profiles on DC subsets suggest that they will respond differently to certain types of pathogens and are functionally diverse.

The presence of a heterogenous DC population with defined locations and cytokine profiles allows for tailoring of the immune response to specific pathogens, while successfully combating foreign pathogens.

\subsubsection{DC function}

DC play a central role in initiating immune responses due to their ability to present antigen and their expression of co-stimulatory molecules, both of which are necessary for an effective $\mathrm{T}$ cell response to be elicited. However, for this to occur DC must interact with T cells within secondary lymphoid organs. Migration of DC to the T cell zones in secondary lymphoid organs is therefore critical to the function of DC. The molecules involved in DC migration, antigen presentation, and co-stimulation are discussed in detail below.

\subsubsection{Migration of DC to secondary lymphoid organs}

Spontaneous maturation or activation through sensing of microbial products results in upregulation of the CC-chemokine receptor 7 (CCR7), which is essential for DC migration from peripheral tissue to secondary lymphoid organs (41, 68-71). CCR7 deficient mice were shown to have markedly defective DC and lymphocyte migration to LN (72). Evidence that DC themselves need to express CCR7 to migrate to $\mathrm{LN}$, rather than other cell types indirectly affecting DC trafficking, came from murine adoptive transfer studies of $\mathrm{CCR} 7^{-/-} \mathrm{DC}$ into $\mathrm{CCR} 7^{+/+}$hosts. Transfer of $\mathrm{CCR} 7^{-/-} \mathrm{DC}$ resulted in reduced recovery of DC from LN compared to transfer of CCR $7^{+/+}$DC (73). However, expression of CCR7 alone is not sufficient for DC migration. A number of additional signals are required to sensitise CCR7 to its ligands, although the signalling mechanisms by which these other mediators alter CCR7 functionality have not been elucidated $(74,75)$. In addition to CCR7, expression of another chemokine receptor, CXCR4, is also upregulated on human DC after 
stimulation (41) and was recently reported to be important in DC migration (76). Taken together these data illustrate that the expression of particular chemokine receptors is important for DC to migrate from the periphery to the T cell areas of secondary lymphoid organs, and present antigen to resident $\mathrm{T}$ cells.

CCR7 has two ligands: CCL21 and CCL19. CCL21 is produced by endothelial cells in lymphatics and high endothelial venules (HEVs) as well as by stromal cells present in the T cell zone of LN. CCL19 is produced by stromal cells and activated DC in the T cell zone (77-80). The current model proposes that $\mathrm{CCR} 7^{+} \mathrm{DC}$ arrive at peripheral lymphatic vessels by responding to a chemotactic gradient of CCL21 and CCL19, originating from the lymphatic vessel. However, there is currently no direct evidence that this is correct, although it is clear that the migration of DC from the periphery to LN depends on CCR7 and its ligands (74).

The classical view is that DC migrate to LN constitutively from peripheral tissues through afferent lymph vessels (74). However, similar to naïve lymphocytes, some DC enter the LN through specialised HEVs. Although $\mathrm{CD}^{+} \mathrm{DC}$ have been proposed to use the hematogenous route (81), attempts to demonstrate directly that $\mathrm{CD}^{+} \mathrm{DC}$ traverse $\mathrm{HEV}$ to enter LN have so far failed (82) but there is evidence indicating that pre-cDCs enter the LN through HEVs, though not specifically $\mathrm{CD}^{+} \mathrm{DC}(83)$. pDC have been reported to enter LN through HEVs. Initially, pDC were shown to accumulate in human inflamed LN just beneath HEVs, suggesting that they may enter LN through this route (84). More recently, intravital microscopy of mouse LN clearly established pDC entered through HEVs (85). It appears that perhaps pDC and $\mathrm{CD} 8^{+} \mathrm{DC}$ can travel to $\mathrm{LN}$ using different routes, which may contribute to their distinct functional roles.

\subsubsection{Antigen presentation}

Immature DC effectively capture and present self and non-self antigens. The molecules involved in the processing and presentation of antigen are discussed in detail below.

\subsection{Major histocompatibility complex (MHC) expression}

There are two main types of MHC molecules: MHC class I (MHC I) and MHC class II (MHC II). All nucleated cells express MHC I molecules, including DC. MHC I is a 
heterodimer consisting of a membrane spanning $\alpha$ chain that associates with a $\beta_{2^{-}}$ microglobulin $\left(\beta_{2} \mathrm{~m}\right)$ molecule. MHC I molecules bind peptides that are usually 8-10 amino acids in length. In contrast, MHC II molecules are expressed only on DC, B cells, macrophages and thymic epithelial cells (86). MHC II molecules consist of $\alpha$ and $\beta$ transmembrane glycoprotein chains and unlike MHC I molecules, MHC II molecules are less restricted by peptide size and bind peptides that are longer than 13 amino acids.

There are two major T cell subsets that are distinguished by expression of the cell surface proteins CD4 and CD8. These two T cell subsets differ in the class of MHC they recognise, with MHC II and MHC I molecules being recognised by $\mathrm{CD}^{+}$and $\mathrm{CD} 8^{+} \mathrm{T}$ cells respectively. Upon recognition of the corresponding MHC/peptide complex and costimulatory molecules (see section 1.1.4.3) on activated $\mathrm{DC}, \mathrm{CD} 4^{+} \mathrm{T}$ cells differentiate into either activated T helper 1 (Th1), Th2, Th17 cells, T follicular helper cells or T regulatory ( $\mathrm{T}$ reg) cells (87-89), whereas $\mathrm{CD}^{+} \mathrm{T}$ cells become effector cytotoxic $\mathrm{T}$ lymphocytes (CTL) (90).

\subsection{CD1 expression}

In addition to MHC I and II, DC express a third class of molecules involved in antigen presentation, the CD1 proteins. Five CD1 genes have been identified in humans that correspond to five proteins, designated CD1a, b, c, d, and e. In contrast, only CD1d has been identified in mice (91). In humans and mice, CD1d is not only expressed on DC, but also macrophages, B cells, thymocytes and peripheral T cells (91-93). The CD1 proteins share sequence homology and overall domain structure with MHC I molecules, being comprised of a heavy chain that is associated with $\beta_{2} \mathrm{~m}$ (94). However, in contrast to MHC I molecules that present peptide antigen, the CD1 family has evolved to present lipids as cognate antigens to NKT cells (95).

\subsection{Presentation on MHC molecules}

The classical model of antigen presentation is based on strict compartmentalization of MHC I and II biogenesis. Peptides generated from the degradation of endogenous proteins are translocated into the endoplasmic reticulum by the transmembrane transporter associated with antigen processing and loaded onto new MHC I molecules. Once 
associated with peptides, MHC I molecules are rapidly transferred through the Golgi apparatus to the cell surface for recognition by $\mathrm{CD}^{+} \mathrm{T}$ cells $(45,90)$. By contrast, exogenous antigen is taken up by DC into endosomes and degraded into polypeptides. Peptides are targeted to lysosome related cellular compartments, which are rich in MHC II molecules, and loaded onto new MHC II molecules, which are then translocated to the cell surface for recognition by $\mathrm{CD}^{+}{ }^{+} \mathrm{T}$ cells. Therefore, MHC I and MHC II present antigen from different intracellular compartments. However, this is not the only mechanism of antigen presentation. Over 30 years ago, work by Bevan demonstrated that priming of $\mathrm{CD}^{+} \mathrm{T}$ cell immune responses in vivo can also occur after presentation of exogenous antigens by MHC I, a phenomenon that he called cross priming (96). Subsequently, the term 'cross presentation' has been used to describe exogenous antigen presentation on MHC I, resulting in activation (cross priming) or tolerisation (cross tolerance) of $\mathrm{CD}^{+} \mathrm{T}$ cells (97).

It is necessary for DC to present exogenous antigens on MHC I to enable efficient T cell activation during a viral infection, in which the DCs themselves are not infected (98). Many studies have shown that DC and macrophages are capable of cross presentation in vitro (reviewed in (97)). However, DC are the main players in cross presentation in vivo (99). Studies using transgenic mice selectively expressing the diphtheria toxin receptor in $\mathrm{CD} 11 \mathrm{c}^{+}$cells showed that selective depletion of $\mathrm{CD} 11 \mathrm{c}^{+} \mathrm{DC}$ prevented $\mathrm{CD} 8^{+} \mathrm{T}$ cell responses to cell associated antigens from Plasmodium yoelii and Listeria monocytogenes (99). Even though DC seem to be the main cell type involved in cross presentation, not all DC subsets have equal capacity to cross present antigens to $\mathrm{CD}^{+} \mathrm{T}$ cells. Bevan's group was the first to report functional differences in the ability to cross present cell associated antigens between the various DC subsets (100). Later studies using fluorescently labelled chicken ovalbumin (ova) as a model antigen, or ova-conjugated fluorescent microspheres to track internalised antigen, revealed that all subsets were capable of antigen uptake, but only the $\mathrm{CD} 8^{+} \mathrm{DC}$ subset could achieve cross priming in vivo (100). $\mathrm{CD} 8^{+} \mathrm{DC}$ were also shown more efficient at cross presenting soluble (101) or latex bead associated antigens (102), and antigens captured by C-type lectin receptors $(103,104)$. In contrast the CD8DC subset appeared more efficient at presenting antigen on MHC II molecules (103, 104).

Cross presentation is an important mechanism for eliciting $\mathrm{CD} 8^{+} \mathrm{T}$ cell responses against viruses that do not infect APC. However, as reviewed by Lin et al, the mechanism by 
which the exogenous antigen accesses the MHC I presentation pathway is not fully elucidated, although several mechanisms have been proposed (97). Recently, the cross presentation of ova protein was shown to require the mannose receptor selectively expressed on $\mathrm{CD}^{+} \mathrm{DC}$ (105). Furthermore, $\mathrm{CD} 8^{+} \mathrm{DC}$ have been found to express higher levels of MHC I processing associated proteins than CD8- DC (104). But further studies are necessary to confirm why the $\mathrm{CD} 8^{+} \mathrm{DC}$ subset is more efficient at cross presentation than other DC subsets (60).

\subsubsection{Co-stimulatory molecules}

By itself, recognition of peptide/MHC complexes expressed on DC is not sufficient for an effective T cell response. Additional signals from co-stimulatory molecules are required to fully activate naïve T cells. DC express many co-stimulatory molecules on their cell surface, which can be divided into two main groups on the basis of their structural configuration, co-stimulatory molecules the immunoglobulin superfamily, and costimulatory molecules of the tumour necrosis factor receptor (TNFR) superfamily.

The most well characterised co-stimulatory molecules are B7-1 (CD80) and B7-2 (CD86), which belong to the immunoglobulin superfamily and are expressed on the cell surface of DC, macrophages and B cells (106). CD86 is constitutively expressed at low levels and rapidly upregulated following activation of DC, whereas CD80 is inducibly expressed and upregulated more slowly than CD86 following activation. Both CD80 and CD86 bind CD28, which is constitutively expressed on mouse T cells (107). Signalling through CD28 lowers the threshold for the activation of naïve $\mathrm{T}$ cells, enhances $\mathrm{T}$ cell proliferation, promotes T cell survival and increases the secretion of cytokines (108-111).

Both CD80 and CD86 can also bind the inhibitory receptor, cytotoxic T lymphocyteassociated antigen 4 (CTLA-4) (112). CTLA-4 expression is rapidly upregulated following $\mathrm{T}$ cell activation and has a higher affinity for both CD80 and CD86 than CD28 (113-116). Signalling through CTLA-4 blocks T cell proliferation and cytokine production $(109,112$, 117). T cell responses go unchecked in the absence of CTLA-4, resulting in fatal lymphoproliferative diseases $(118,119)$. This suggests that CTLA-4 plays an important negative regulatory role in $\mathrm{T}$ cell activation and in maintaining homeostasis. 
The TNFR superfamily co-stimulatory molecule CD40 is expressed on B cells, macrophages, DC (120) and some T cells (121). CD40 on DC binds to CD40L, which is expressed on activated T, NK, NKT cells, platelets, eosinophils and mast cells (120, 122, 123). Engagement of CD40 activates DC leading to IL-12 production and the upregulation of co-stimulatory molecules required for $\mathrm{T}$ cell activation and differentiation (124). In particular, CD40 ligation drives increased expression of B7 co-stimulatory molecules. Furthermore, ligation of CD40 on DC is reported to be sufficient to provide CD4 ${ }^{+} \mathrm{T}$ cell mediated help for $\mathrm{CD}^{+} \mathrm{T}$ cell responses (125-127). Additionally ligation of CD40 has also been shown to enhance DC survival by the upregulation of anti-apoptotic molecules and expression of serine protease inhibitor (SPI)-6 (128, 129).

Another TNFR superfamily co-stimulatory molecule, Tumour necrosis factor (TNF)related activation-induced cytokine (TRANCE), expressed by activated T cells, also enhances the survival of DC and stimulates the secretion of cytokines required for $\mathrm{T}$ cell differentiation when it binds its receptor, TRANCE-R on activated DC (130-132).

Additional co-stimulatory molecules of the immunoglobulin superfamily, such as inducible co-stimulator ligand (ICOS-L) and programmed death-1 ligand (PD-L1), along with OX40L, 4-1BBL and CD70 of the TNFR super family are also present on DC. These costimulatory molecules enhance $\mathrm{T}$ cell clonal expansion, cytokine production and survival upon binding their respective receptors on T cells (133-141). However, as these molecules are not the focus of this thesis they will not be discussed in any further detail.

\subsubsection{Regulation of DC survival}

The central role played by DC in initiating adaptive immune responses means their survival is critical in the generation of an effective adaptive immune response and hence the outcome of the immune response. There are a number of mechanisms that regulate DC survival, and this DC survival affects the $\mathrm{T}$ cell response in either a positive or negative way. These are discussed below. 


\subsubsection{Mechanisms of DC survival and regulation}

Survival of DC may be intrinsically determined or it may be regulated by external factors such as signals from pathogens. Several studies have investigated the lifespan of steady state DC subsets in vivo by using bromodeoxyuridine (BrdU) labelling. Mouse splenic DC subsets had a rapid turn over (half-life 1.5-2.9 days), with $\mathrm{CD} 8^{+} \mathrm{DC}$ turning over more rapidly than the CD8 ${ }^{-}$DC subset $(81,142,143)$. This suggests that DC survival may be intrinsically controlled to some extent. However, engagement of TLRs has been shown to affect the survival of DC. Treatment of BMDC with a range of TLR ligands led to an altered DC lifespan depending on the TLR ligand (144). Unmethylated CpG motifs, the ligands for TLR9, enhanced DC survival and increased expression of the anti-apoptotic molecules Bcl-2 and Bc1-xL. LPS and lipoteichoic acid, which are TLR4 and TLR2 ligands respectively, initially enhanced DC survival. However, after 3 days the cells underwent increased cell death associated with decreased expression of Bcl-2 and Bc1-xL (144). In vivo administration of LPS led to DC maturation and migration from the spleen marginal zone to the $\mathrm{T}$ cell zone, resulting in an increased $\mathrm{DC}$ turnover rate $(61,81,145)$. Ligation of certain TLR has been proposed to set a 'molecular timer' that limits the lifespan of DC and controls the homeostasis and immunogenicity of these cells (144). These results suggest that both intrinsic and extrinsic factors play a role in the regulation of DC survival.

The co-stimulatory molecules, CD40L and TRANCE, expressed by activated T cells (124, 130-132) can also enhance DC survival. Upon engagement of its receptor on mature DC, TRANCE has been shown to inhibit apoptosis of both mouse and human DC in vitro (131, 132). Ligation of CD40 on human DC also enhances DC survival due to resistance to Fasinduced and spontaneous apoptosis $(128,146)$. Furthermore, DC lacking CD40 expression or treatment of mice with an anti-CD40L mAb resulted in the decline of total DC overtime, whereas treatment with a CD40 agonist resulted in prolonged longevity in vivo (147). It has been suggested that the enhanced survival of DC after either ligation of TRANCE or CD40 is due to the upregulated expression of the anti-apoptotic molecules Bcl-xL and Bcl$2(128,132)$.

CTL, NK and NKT cells have been proposed to play a role in regulating DC survival. As reviewed by Degli-Esposti, a number of studies have found that immature DC are susceptible to NK cell mediated cytolysis (148), via signalling through TNF-related 
apoptosis-inducing ligand (TRAIL) (149). NKT cells have also been shown to kill DC in vitro by a number of mechanisms (150-152), which will be discussed further in section 1.4. CTL have been shown to eliminate antigen loaded DC (153-155) in a perforin-dependent manner $(155,156)$, which will also be discussed further in section 1.4.

\subsubsection{Effect of DC survival on $\mathrm{T}$ cell responses}

$\mathrm{T}$ cell activation involves extended contact between $\mathrm{T}$ cell and DC, therefore increased DC survival may affect $\mathrm{T}$ cell responses in a positive or negative way. Positive effects have been observed when DC survival was improved by manipulating the expression of anti- or pro-apoptotic molecules. Altering the expression of Bcl-2 and Bcl-xL (144, 157-161) or using small interfering RNA (siRNA) to silence gene expression of the pro-apoptotic molecules, Bak and Bax, in DC in vivo (162), resulted in enhanced antigen-specific CD4 ${ }^{+}$ and $\mathrm{CD}^{+} \mathrm{T}$ cell responses and anti-tumour effects. In contrast, negative effects have also arisen with increased DC survival. Humans with a defect in caspase 10 (163) or transgenic mice with a targeted inhibition of apoptosis in DC by expression of a caspase inhibitor (164) have an accumulation of DC in LN, and also show spontaneous T cell activation but this is correlated with systemic autoimmunity.

In summary, several mechanisms exist that regulate DC survival. Increased DC survival enhances $\mathrm{T}$ cell responses, which has been associated with both positive and negative immune responses. Thus the regulation of DC survival plays a critical role in establishing an effective and desirable immune response.

\subsubsection{DC-based cancer vaccines}

DC can be used as a tool for increasing anti-cancer immune responses. Early studies demonstrated that DC could be cultured in large numbers from bone marrow precursors $(165,166)$. A common approach is to inject cancer patients with their own DC loaded ex vivo with tumour antigens. A number of mouse studies (167) and early clinical trials testing vaccination with ex vivo generated DC loaded with tumour antigens provided proof of principle that therapeutic immunity can be achieved (168). Mouse studies in our group have also shown that DC loaded with tumour antigen can induce protective anti-tumour immunity (169). Yet, after multiple clinical trials, the clinical benefit measured by 
regression of established tumours has been observed in only a fraction of patients (167, $168,170)$. Thus, a better understanding of DC biology and the requirements for a protective DC-mediated anti-tumour immune response will enable the generation of more efficient DC-based cancer vaccines.

\section{$1.2 \mathrm{CD8}^{+} \mathrm{T}$ cells}

\subsection{1 $\mathrm{CD8}^{+} \mathrm{T}$ cell development}

$\mathrm{CD}^{+} \mathrm{T}$ cells are generated in the thymus from lymphoid precursor cells that have migrated from the BM. Two distinct lineages of T cells, $\alpha \beta$ and $\gamma \delta$, are identified based on the type of $\mathrm{T}$ cell receptor (TCR) expressed. The $\mathrm{CD}^{+} \mathrm{T}$ cells develop from the $\alpha \beta$ lineage. $\mathrm{CD}^{+} \mathrm{T}$ cells undergo a developmental selection process within the thymus (171). They are first positively selected by thymic cortical epithelial cells, and then negatively selected by APC such as DC and macrophages, based on their ability to recognise selfantigens presented in the context of MHC I molecules. T cells are deleted either when they fail to be positively selected if their TCR has a very low affinity for MHC-self antigen complexes, or when they undergo negative selection if they have a very high affinity for self-antigen (172). Once the $\mathrm{CD} 8^{+} \mathrm{T}$ cells have completed the developmental process within the thymus they leave the thymus and traffic through the lymphatic system to populate the secondary lymphoid organs.

\subsubsection{Activation and differentiation of naïve $\mathrm{CD8}^{+} \mathrm{T}$ cells}

Upon activation by DC expressing MHC I and peptide, naïve $\mathrm{CD}^{+} \mathrm{T}$ cells go through 3 phases of differentiation: expansion, contraction and memory development $((173,174)$, Figure 1.3). The first stage, expansion, is initiated in the secondary lymphoid tissues where $\mathrm{CD}^{+} \mathrm{T}$ cells encounter their specific antigen. $\mathrm{CD}^{+} \mathrm{T}$ cells clonally expand and differentiate into CTL, leading to a substantial increase ( 1000-fold) in the precursor frequency of antigen specific $\mathrm{T}$ cells in immune animals compared with naïve animals (175-178). Clonal expansion ensures that there are enough antigen specific $\mathrm{T}$ cells to clear the invading pathogen. The second stage is the contraction phase in which the number of 
cells declines and typically only $5-10 \%$ of the antigen specific T cells remain. During the memory phase, long-lived antigen specific cells are present and survive long-term, ready to elicit a faster and stronger immune response upon pathogen re-challenge.

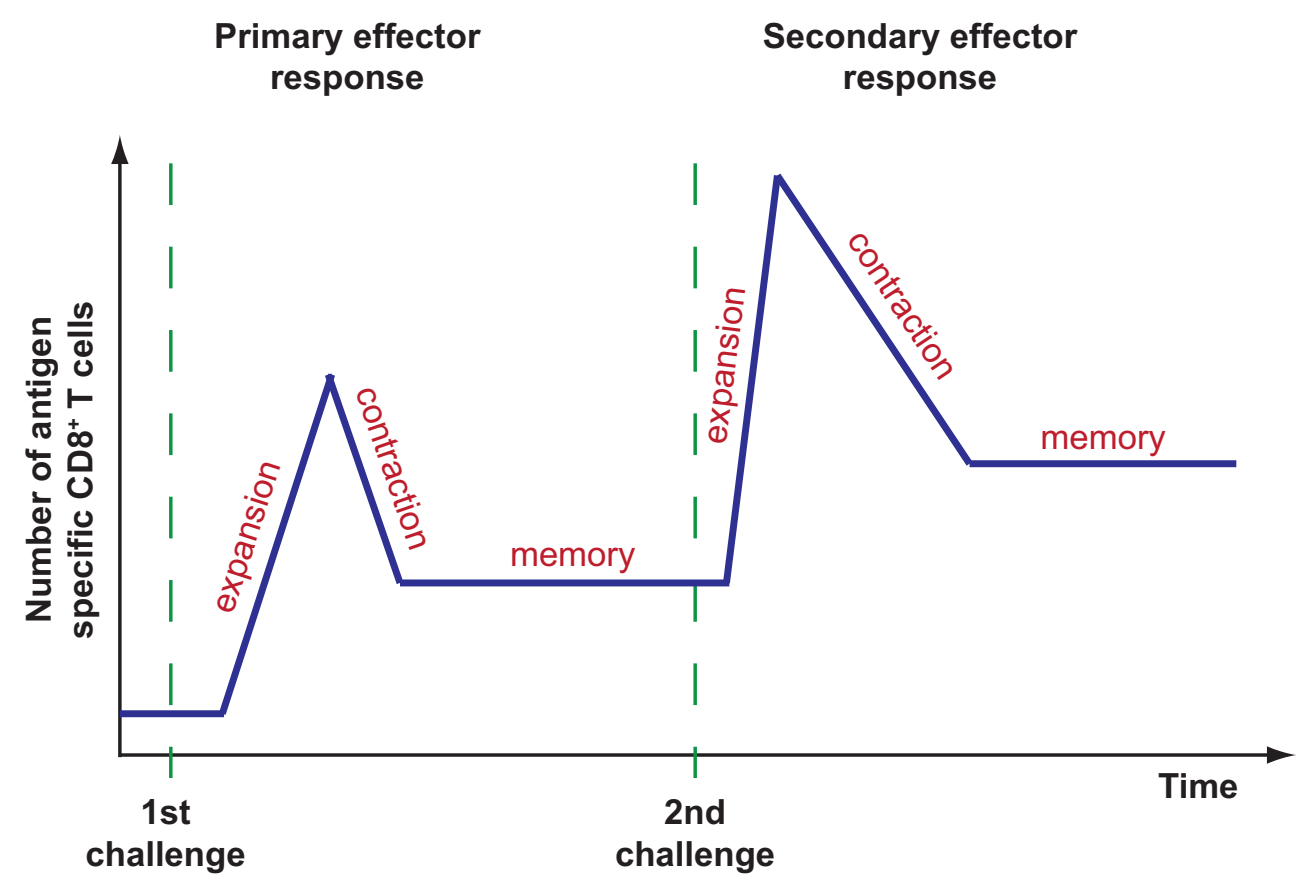

Figure 1.3 The 3 phases of naïve $T$ cell differentiation

A schematic diagram of naïve $\mathrm{CD} 8^{+} \mathrm{T}$ cell differentiation following host infection with a pathogen such as influenza A virus. After a small lag period naïve precursor T cells undergo many rounds of cell division and generate millions of effector cytotoxic T lymphocytes (CTL) by about 7-8 days post-infection. Then, most effectors die, leaving behind a pool of memory $\mathrm{CD} 8^{+} \mathrm{T}$ cells. Upon reinfection, memory $\mathrm{CD} 8^{+} \mathrm{T}$ cells are able to proliferate more rapidly than the naïve precursors but still undergo a contraction phase where a large majority of cells die.

\subsubsection{Expansion phase of naïve $\mathrm{CD8}^{+} \mathrm{T}$ cells}

As mentioned in section 1.1.4.3, two distinct signals are required for optimal expansion and differentiation of resting $\mathrm{CD}^{+} \mathrm{T}$ cells into cytotoxic $\mathrm{T}$ lymphocytes (CTL). The first signal is delivered via stimulation of the T cell receptor (TCR) by peptide-MHC complexes displayed on the surface of APCs. The second signal is co-stimulatory, such as 
CD80/CD86-CD28 interactions and serves to amplify or modify the first signal. The most potent activators of naïve $\mathrm{CD}^{+} \mathrm{T}$ cells are $\mathrm{DC}$, because they provide both signals to $\mathrm{T}$ cells.

In addition to signal 1 and 2, there is evidence that optimal clonal expansion and development of effector function require a third signal (179). A study using artificial APC showed that IL-12 was necessary for activating naïve T cells (180). In vivo studies using transgenic $\mathrm{T}$ cells also demonstrate that $\mathrm{IL}-12$ acts as a third signal directly on naïve $\mathrm{T}$ cells $(181,182)$. IL-12 has been found to regulate the survival of activated CD8 ${ }^{+} \mathrm{T}$ cells through altering the expression of the anti-apoptotic molecule, Bcl-3 (183). Type I interferons (IFNs) were also shown to provide the third signal (184) by directly signalling to $\mathrm{CD}^{+} \mathrm{T}$ cells to promote their survival and effector differentiation during expansion (184-186). DC produce both IL-12 and type I IFNs upon stimulation with TLR ligands (63) and in response to CD40-CD40L interactions with $\mathrm{CD}^{+}{ }^{+} \mathrm{T}$ cells and NKT cells (125127, 187). Taken together these data indicate that the presence of IL-12 and type I IFNs are important in providing a third signal for optimal development of effector $\mathrm{CD}^{+} \mathrm{T}$ cells.

Other studies have focussed on determining the length of antigenic stimulation required for activating naïve T cells. Only a brief period of antigenic stimulation (2-24 hr) was shown to be enough for naïve $\mathrm{CD} 8^{+} \mathrm{T}$ cells to commit to 7-10 cell divisions and differentiate into CTL (188-191). Moreover, once naïve T cells received an activation signal they continued to divide and differentiate in the absence of further antigenic stimulation, suggesting that activated $\mathrm{T}$ cells are programmed to clonally expand and differentiate into effector cells (188). Therefore it would appear that after only a short period of antigenic stimulation naïve $\mathrm{CD} 8^{+} \mathrm{T}$ cells can clonally expand and generate an effective CTL response.

Interactions between naïve T cells and DC in vivo have been the subject of several studies in recent years. This has been made possible by two-photon laser scanning microscopy which allows real time imaging of T cell-DC interactions within a LN (192). Von Andrian and colleagues were the first to carefully examine the evolution of $\mathrm{CD} 8^{+} \mathrm{T}$ cell-DC contact dynamics over time (193). They found that peptide-loaded DC primarily established transient interactions during the first $8 \mathrm{hr}$ in the LN. It was only after this initial phase that stable T cell-DC contacts were detected (193). Other studies have also observed initial short-lived interactions, whereas other authors failed to observe this initial phase of 
transient interactions (reviewed in (194)). It is thought that the differences observed could be explained by the diversity of experimental conditions used in the different studies. Nevertheless, the emerging consensus is that strong signals induce rapid and stable interactions, while weaker signals lead to transient T cell-DC interactions. Differences in T cell-DC contacts might contribute to creating a heterogeneous T cell response (194). Thus, improvements in technology have helped gain a better understanding into the in vivo interactions that occur between the T cell and DC.

\subsubsection{Contraction of $\mathrm{CD8}^{+} \mathrm{T}$ cells}

After extensive proliferation of antigen specific $\mathrm{CD}^{+} \mathrm{T}$ cells following an infection or vaccination, a contraction phase occurs (Figure 1.3). In general, the contraction phase eliminates $\sim 90-95 \%$ of antigen-specific effector $\mathrm{CD}^{+} \mathrm{T}$ cells $(195,196)$. Despite this massive elimination, the antigen-specific $\mathrm{T}$ cell population is maintained at a higher frequency than they were in the initial naïve precursor population. These surviving cells are memory $\mathrm{CD}^{+} \mathrm{T}$ cells. Originally it was thought that the onset of the contraction phase correlated with the kinetics of pathogen clearance (173). However, experiments investigating the kinetics of the contraction phase have found it to be programmed early during infection $(188,190,195)$. In experiments where the duration of infection was limited by antibiotic treatment or allowed to persist through the use of PKO mice, contraction kinetics were not affected (195). The same contraction kinetics were also observed regardless of differences in the initial expansion and were independent of the duration of infection and antigen persistence $(188,190)$. These results suggest that contraction of antigen-specific $\mathrm{CD}^{+} \mathrm{T}$ cells occurs independently of pathogen clearance and is a programmed event.

\subsubsection{Memory $\mathrm{CD8}^{+} \mathrm{T}$ cells.}

Memory $\mathrm{CD}^{+} \mathrm{T}$ cells are long-lived cells that persist in a resting state after the resolution of infection and serve to protect against a secondary challenge. Memory $\mathrm{CD} 8^{+} \mathrm{T}$ cells are heterogeneous in their phenotype, location and effector functions.

In the late 1990s, two subsets of memory cells from human peripheral blood were defined: effector memory $\left(\mathrm{T}_{\mathrm{em}}\right)$ cells and central memory $\left(\mathrm{T}_{\mathrm{cm}}\right)$ cells (197). $\mathrm{T}_{\mathrm{cm}}$ and $\mathrm{T}_{\mathrm{em}}$ can be distinguished based on expression of the LN homing receptors, CD62L and CCR7, effector 
functions and proliferative ability. $\mathrm{T}_{\mathrm{em}}$ cells are defined as $\mathrm{CD} 62 \mathrm{~L}^{\mathrm{lo}}$ and $\mathrm{CCR} 7^{\mathrm{lo}}$, possess immediate effector function and are found in the peripheral tissues poised to rapidly clear pathogens. In contrast, $\mathrm{T}_{\mathrm{cm}}$ cells are CD62 $\mathrm{L}^{\mathrm{hi}}$ and $\mathrm{CCR} 7^{\text {hi }}$, exist mainly in $\mathrm{LN}$ and express effector function upon restimulation (197). These memory cell subsets have been characterised in mice as well as in humans, (198-200). However, it is now evident that memory $\mathrm{T}$ cell heterogeneity is more complex than simply $\mathrm{T}_{\mathrm{cm}}$ and $\mathrm{T}_{\mathrm{em}}$ cells (201).

Currently, there is ongoing debate in the literature about the development and lineage relationships of memory $\mathrm{CD}^{+} \mathrm{T}$ cell subsets. A number of models have been proposed including the linear and divergent models (202). However, these models may not be mutually exclusive and multiple pathways may exist depending on the $\mathrm{T}$ cell priming conditions (201). The main difference between the proposed models is whether memory cells are required to pass through an effector phase. There is some indication that memory cells are direct descendents of effector cells (203-205), while other evidence supports a divergent lineage, whereby memory cells directly differentiate from naïve cells without passing through an effector phase (206-210).

Whilst the differentiation pathway of memory $\mathrm{CD} 8^{+} \mathrm{T}$ cells has not been completely elucidated, memory cells are required for long-term protective immunity. Memory $\mathrm{CD}^{+} \mathrm{T}$ cells rapidly respond to re-infection and can clear a second infection faster than naïve $\mathrm{CD}^{+} \mathrm{T}$ cells can. This is due to the increased precursor frequency of antigen specific $\mathrm{T}$ cells in immune animals $(175-178,211)$ and the capacity to produce larger amounts of interferon-gamma (IFN- $\gamma$ ), and faster production of perforin compared to naïve $\mathrm{CD}^{+} \mathrm{T}$ cells $(177,188,212-217)$. In addition, unlike naïve $\mathrm{CD}^{+} \mathrm{T}$ cells which are found in $\mathrm{LN}$, effector or certain memory $\mathrm{CD} 8^{+} \mathrm{T}$ cell subsets are able to patrol the peripheral tissues, enabling earlier encounter with pathogens (218). These features of memory CD8 ${ }^{+} \mathrm{T}$ cells make them distinct from naïve $\mathrm{CD}^{+} \mathrm{T}$ cells and enable them to mediate long-term protective immunity.

\subsubsection{Effector functions of activated $\mathrm{CD8}^{+} \mathrm{T}$ cells}

Upon activation, $\mathrm{CD}^{+} \mathrm{T}$ cells differentiate into CTL and develop effector functions, including the ability to produce cytokines to recruit other cells to the site of infection and the ability to directly interfere with pathogen replication (196). CTL are able to induce 
killing of infected cells by two distinct pathways: death receptor-mediated apoptosis and cytotoxic granule exocytosis (219).

\subsubsection{Cytokine production}

Upon activation, CTL secrete cytokines that can have both local and systemic consequences. CTL secrete IFN- $\gamma$ and tumour necrosis factor alpha (TNF- $\alpha$ ), which both contribute to their cytotoxic function. TNF- $\alpha$ can bind to its receptor, TNFR, which is present on the cell surface of target cells to induce programmed cell death (220). The presence of TNF- $\alpha$, along with IFN- $\gamma$, can lead to macrophage activation and recruitment to sites of infection. Experiments have also shown that direct exposure to IFN- $\gamma$ and TNF$\alpha$ induces cellular proteins that can decrease transcription and/or replication of several viruses $(221,222)$. IFN- $\gamma$ also induces the expression of MHC I on infected cells, which increases the chance that infected cells will be recognised as target cells for cytotoxic attack $(221,222)$. In addition, activated $\mathrm{CD}^{+} \mathrm{T}$ cells secrete IL-2, which helps drive their proliferation and differentiation.

\subsubsection{Fas-FasL}

One mechanism of cell lysis utilised by CTL is via cell death receptors such as Fas (CD95), TNFR-1 and TRAIL receptor (TRAIL-R) (223). Expression of death receptors on the target cell can lead to cell destruction by CTL expressing the corresponding ligand. The best-characterised death receptor pathway is Fas-FasL. Fas is expressed on many cells, whereas FasL is expressed predominantly on activated $\mathrm{CD} 8^{+} \mathrm{T}$ cells due to its upregulation upon TCR stimulation (224). Engagement of Fas with FasL results in classical caspasedependent apoptosis. The Fas-FasL pathway is involved not only in cell-mediated cytotoxicity but also in $\mathrm{T}$ cell homeostasis $(225,226)$.

\subsubsection{Perforin and the perforin/granzyme cytotoxic pathway}

In addition to death receptor mediated apoptosis, the main pathway that is utilised by CTL to kill target cells is the perforin/granzyme pathway. Perforin was first described in 1985 and was characterised as a calcium-dependent, pore-forming cytolytic protein (227-229). Perforin is a $\sim 67 \mathrm{kDa}$ multidomain protein with some similarity to the pore forming 
component of complement, C9 $(230,231)$. However, until recently the perforin domains were largely undefined and the protein structure was not solved, due to the lack of a recombinant form of perforin (232). Detailed mutational and structure-function analysis has revealed that the highly conserved amphipathic $\alpha$ helical region, formerly thought to constitute the membrane-spanning domain (233), is involved in the assembly of perforin monomers into a pore complex (234). It is anticipated that future studies will completely solve the protein structure and define the domains of perforin, which will provide insights into its function.

Perforin is primarily expressed in NK cells and antigen-experienced $\mathrm{CD} 8^{+} \mathrm{T}$ cells, but it can also be expressed in subpopulations of $\mathrm{CD}^{+}{ }^{+} \mathrm{T}$ cells and NKT cells (235-237). Perforin, along with granzymes that are complexed with the proteoglycan serglycin, are sequestered into specialised secretory cytotoxic granules (238). Granzymes are highly specific proteases; granzyme A and in particular granzyme B are the most abundant granzymes in mice and humans. The granzymes are redundant, each capable of proteolytically activating independent cell death pathways (239). In CTL, the cytotoxic granules are synthesised only after the cell has encountered specific antigen and becomes activated (240, 241), whereas in NK cells, cytotoxic granules are formed during development $(237,242)$. Recognition of a target cell by a CTL triggers rapid polarisation of the microtubule organising centre towards the immunological synapse, followed by the granules moving along the microtubules in the direction of the plasma membrane. After the cytotoxic granules have been polarised at the cell membrane, they are secreted into the immunological synapse in an ordered set of events. This results in the rapid delivery of the contents of cytotoxic granules to a precisely defined point between the CTL and target cell (243). Subsequently, the granzymes are delivered to the target cell resulting in cell death. The mechanism of how perforin delivers the granzymes to the target cell is still not fully understood. However, the generation of PKO mice has demonstrated the absolute requirement of perforin for granzyme-dependent killing (244-248).

\subsubsection{Proposed models for perforin-mediated delivery of granzymes into target cells}

A number of models have been proposed to explain how perforin facilitates the delivery of granzymes to the target cell. A series of experiments where perforin and granzymes were 
over-expressed in the mast cell line RBL demonstrated that both perforin and granzymes were required for the DNA damage observed during CTL killing $(249,250)$. These results formed the basis for the first proposed mechanism of perforin/granzyme-mediated cytotoxicity. Perforin was proposed to form a plasma membrane pore to act as a channel, through which the granzymes could pass (Figure $1.4 \mathrm{~A}$ ). However, perforin pores may be too small to allow passage of molecules as large as granzymes because small dyes that ought to be able to pass through perforin sized pores are unable to enter perforin treated cells $(251,252)$. However, a later study, using sublytic doses of perforin, demonstrated that perforin does form cell membrane pores and small amounts of molecular dyes were able to enter the cell (253). The earlier experiments may have failed to observe the dye present in the cytosol because not much dye enters and it does not diffuse throughout the cytoplasm (254). This original model of pore-forming perforin was further questioned after studies showed that granzyme B was taken up into target cells in a perforin-independent manner, via the mannose-6-phosphate receptor (255). This mechanism of granzyme uptake has been contested by a number of groups (256-258) and therefore the exact mechanism of perforin action remains to be elucidated.

A modification to the original pore forming model has been proposed by Froelich and colleagues, in which perforin forms pores in the endosomal membrane rather than in the plasma membrane, releasing granzymes from endosomes into the cytosol $((251,252)$, Figure 1.4 B). This idea is supported by the fact that transfer of granzyme B into target cell endosomes occurred in a perforin-independent manner, but that perforin was required for endosomal disruption, leading to apoptosis $(251,259)$. However, there was no evidence of perforin in the target cell cytosol.

More recently, another modification to the original model by Lieberman and colleagues proposes that perforin forms small pores in the cell membrane that trigger a $\mathrm{Ca}^{2+}$ influx leading to a membrane repair response and endocytosis of granzymes and perforin into giant endosomes, followed by perforin mediated release of granzymes into the cytosol ((253), Figure 1.4 C). However, further evidence is still required to fully validate this latest model. Regardless of which model is correct, all point to perforin forming a membrane pore. Nevertheless, further investigations ought to elucidate its precise mechanism of action. 


\section{Figure 1.4 Models of perforin-dependent delivery of granzymes to the target cell}

(A) The original pore model proposed that perforin formed a pore in the plasma membrane, through which granzymes could diffuse and access key substrates leading to apoptotic death. (B) The endosomolysis model proposes that granzymes can be taken up in a perforin-independent manner but that perforin is required for endosomal disruption. (C) The most recent model proposes that perforin forms small pores in the plasma cell membrane that trigger a $\mathrm{Ca}^{2+}$ influx, which activates a membrane repair response in which internal vesicles such as lysosomes donate their membranes to patch the holes. The next step involves endocytosis of granzymes and perforin into endosomes, followed by perforin mediated release of granzymes in to the cytosol leading to cell death. Adapted from Trapani and Smyth (260) and Keefe et al (253). 
(A) Original pore model

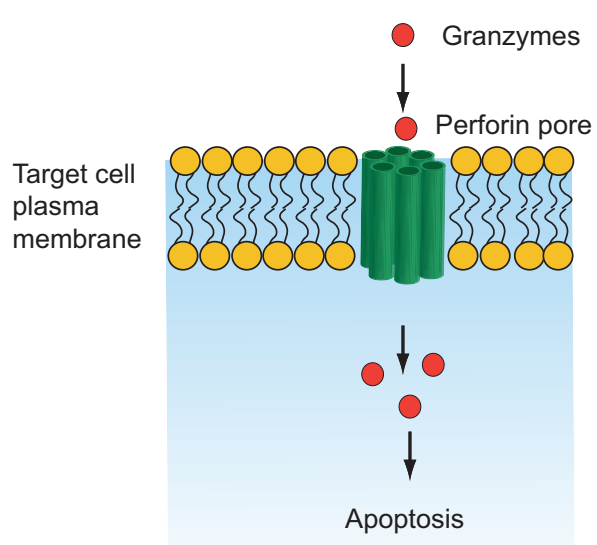

(B) Endosomolysis model

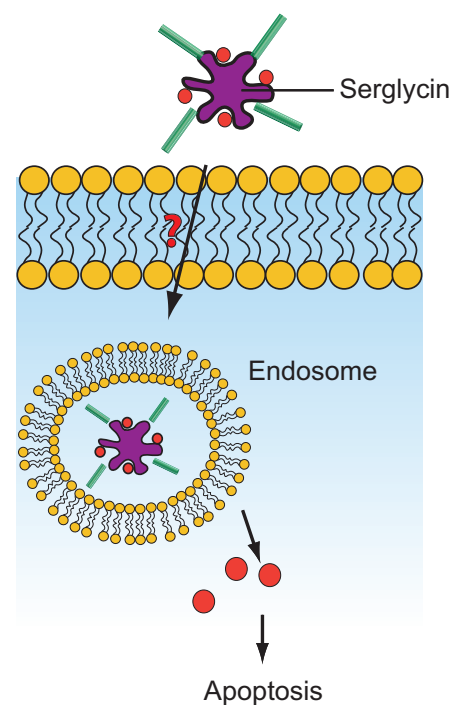

(C) Wound healing model

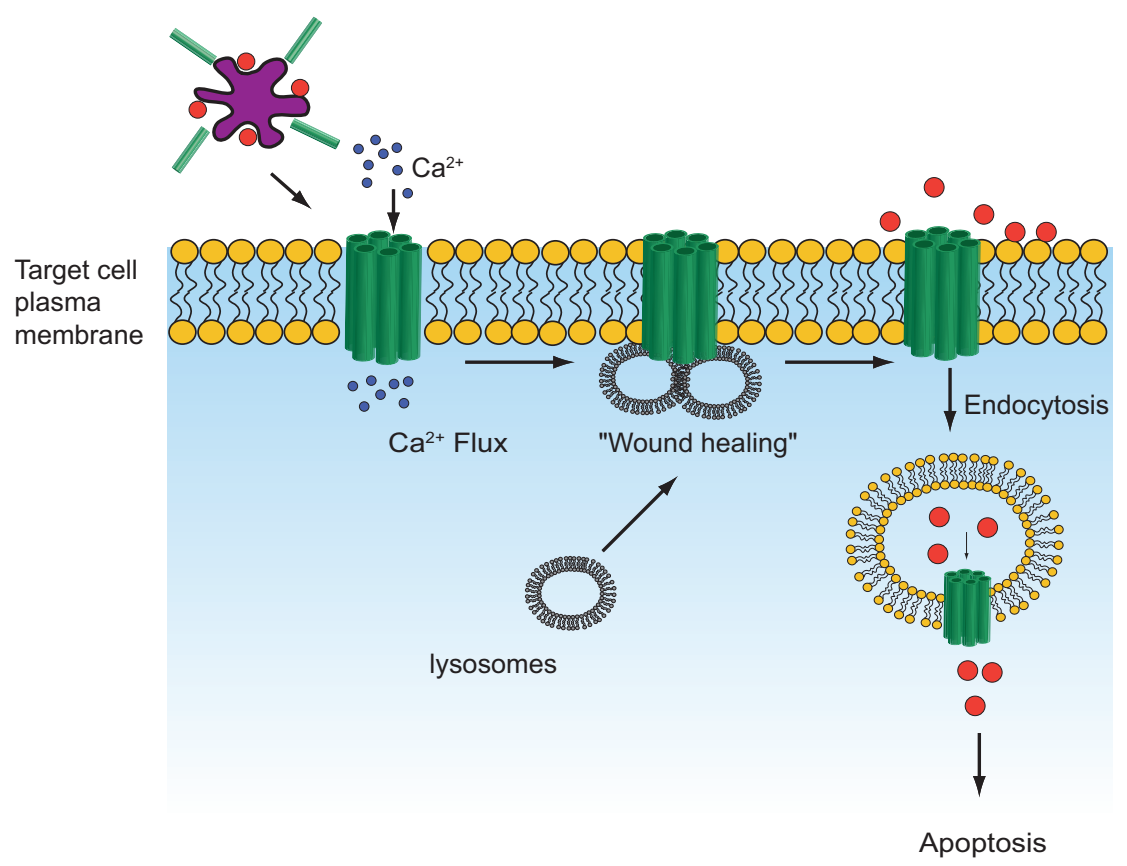

Key:
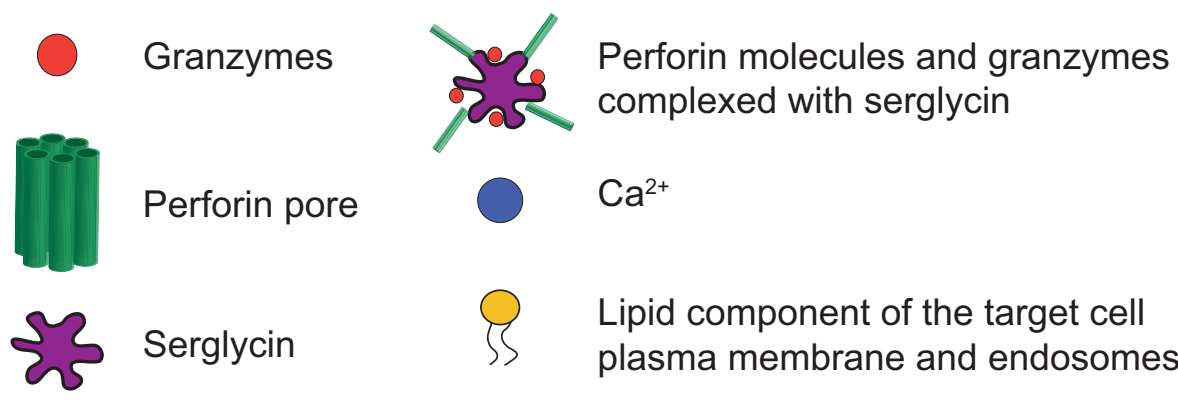

nymes complexed with serglycin 


\subsection{Natural Killer T (NKT) cells}

The term 'NKT cells' was first coined in 1995 to define a novel subset of mouse T cells characterised by cell surface expression of NK1.1 and an $\alpha \beta$ TCR, with V $\alpha 14$ incorporated in the $\alpha$ chain ((261), Figure 1.5). More recently, murine NKT cells have been defined as cells expressing an invariant V $\alpha 14 \mathrm{~J} \alpha 18$ TCR. These 'invariant' type I NKT cells are reactive to the glycosphingolipid $\alpha$-Galactosyleramide ( $\alpha$-GalCer) presented on MHC I-like CD1d molecules (262). In humans CD1d-restricted, $\alpha$-GalCer reactive cells are also found and express a V $\alpha 24$ J $\alpha 18$ TCR (263). The CD1d restriction can be used to identify NKT cells by using $\alpha$-GalCer loaded CD1d tetramers that specifically bind the TCR of NKT cells (264266), these cells are referred to as "iNKT". A second subset of CD1d-restricted, type II NKT cells has been identified, however, they are not reactive to $\alpha$-GalCer and have a diverse TCR $\alpha$ repertoire (267). These NKT cells are less well characterised, mainly because they cannot be identified using $\alpha$-GalCer loaded CD1d tetramers. From here on in the term NKT cell will refer to the 'invariant' type I NKT cells.

An important feature of NKT cells is their ability to rapidly produce of a wide range of cytokines; within hours of stimulation NKT cells produce IL-4, IFN- $\gamma$ and TNF- $\alpha(268,269)$. The ability of NKT cells from naïve mice to rapidly produce cytokines, may be due to the presence of high levels of preformed IL- 4 and IFN- $\gamma$ mRNAs expressed even in a resting state $(270,271)$. To date, NKT cells have been shown to produce IFN- $\gamma$ and IL-4, as well as interleukins $-2,-5,-6,-10,-13,-17,-21$, TNF- $\alpha$, Transforming growth factor $\beta$ (TGF $\beta$ ) and GM-CSF in addition to a range of chemokines (272). It is through their production of this diverse array of cytokines and chemokines that NKT cells have been implicated in many different immune responses, from autoimmune diseases to asthma and infectious diseases along with tumour protection $(263,273-276)$. 


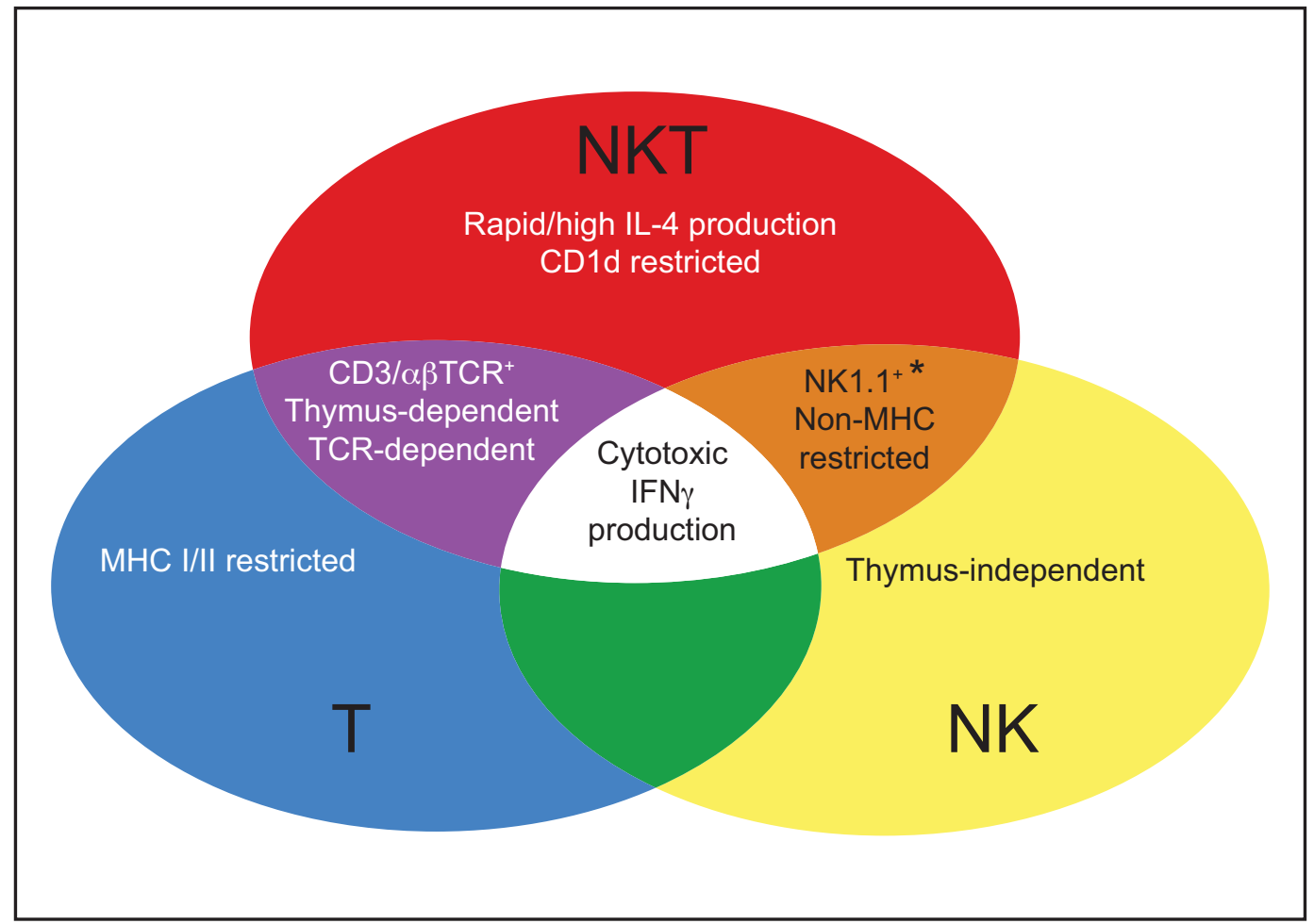

Figure 1.5 Mouse NKT cells share some characteristics of NK and T cells

This Venn diagram shows the key features of NKT, T and NK cells. Characteristics that are unique to each lineage are shown in the non-overlapping sections, whilst common characteristics are indicated in the overlapping areas. The central white area indicates the characteristics exhibited by all three lineages. * NK1.1 is not present in all mouse strains. Reproduced from Godfrey et al (273).

\subsubsection{NKT cell development}

NKT cells are a thymus-derived T cell subset, but are developmentally and functionally distinct from conventional $\mathrm{CD} 4^{+}$and $\mathrm{CD}^{+} \mathrm{T}$ cells (277-280). NKT cells separate from conventional T cell development at the double positive (DP; $\mathrm{CD} 4^{+} \mathrm{CD} 8^{+}$) thymocyte stage (280-283). In contrast to conventional T cells, which are positively selected by thymic cortical epithelial cells, NKT cells are positively selected by CD1d expressing DP cortical thymocytes $(277,284,285)$. Whether NKT cells are subjected to negative selection is not yet clear. There is some evidence that negative selection does occur; however, no study has directly 
demonstrated the deletion of pre-formed NKT cells $(277,284-286)$. Thus, although much has been learnt about NKT cell development over the last few years there are still areas that require further investigation to elucidate the full developmental pathway of NKT cells.

Within the thymus, NKT cell precursors undergo a series of differentiation steps that have been defined by the differential expression of CD24, CD44, DX5 and NK1.1 (286, 287). The final maturation step, which results in the expression of NK1.1, can occur in both the thymus and periphery. Most of the NKT cells that emigrate from the thymus are immature, and complete their maturation in the periphery $(280,288,289)$. Some mature thymic NKT cells do migrate to the periphery, while others remain as long-term thymus resident cells (289). Mature NKT cells in the periphery are widely distributed throughout the body; they are most abundant in liver, BM and thymus, while they represent a smaller proportion of $\mathrm{T}$ cells in the spleen, LN, blood and lung $(263,264,266,273,276)$. In the periphery, mature NKT cells have a semi-activated phenotype, resembling activated $\mathrm{CD} 8^{+} \mathrm{T}$ cells $(290,291)$. This semi-activated phenotype could be responsible for the rapid activation of NKT cells that results in the production of a large array of cytokines.

\subsubsection{NKT cell subsets}

NKT cells can be divided into subsets based on the expression of the co-receptor CD4. In mice they can be either $\mathrm{CD}^{+}$or double negative $\left(\mathrm{DN}\right.$; $\left.\mathrm{CD}^{-} \mathrm{CD}^{-}\right)(264,266)$, whereas in humans an additional subset exists that is positive for the co-receptor CD8 (235). It has been proposed that these subsets are functionally distinct and play different roles in immune responses (235, 292, 293). The strongest evidence is provided by a study by Crowe et al (292) showing that the CD4 liver NKT cells elicit superior tumour protection compared to CD4 ${ }^{+}$NKT cells. In addition, many cytokines are differentially expressed by NKT cell subsets (294). Particularly in humans, there is strong evidence that the $\mathrm{CD} 4^{+}$NKT cells are enriched for Th2 cytokines $(235,293,295)$. Additionally, human NKT cell subsets have been found to differentially regulate DC activity (296). CD4 ${ }^{+} \mathrm{NKT}$ cells induced IL-12 production by DC, while CD4 ${ }^{-}$ NKT cells were found to eliminate DC by cytolysis and cause down regulation of IL-12 production. This study did not investigate the mechanism of DC killing by NKT cells but an earlier study did demonstrate that human NKT cell subsets differentially express molecules 
involved in cytotoxicity (235). Human CD4 ${ }^{-}$NKT cells expressed more perforin compared to the $\mathrm{CD} 4^{+}$NKT cells, which expressed a greater amount of intracellular FasL than the CD4NKT cells (235). Taken together, these results from both human and mouse studies provide evidence that functionally distinct subsets of NKT cells may play different immunoregulatory roles.

\subsubsection{NKT cell ligands}

\subsubsection{1 $\alpha$-Galactosylceramide ( $\alpha$-GalCer)}

Kirin Pharmaceuticals identified the first NKT cell agonist during screenings for natural anticancer medicines. Extracts from Agelas mauritianus, a marine sponge, prolonged survival of mice bearing B16 melanoma (297). The active component was identified as an $\alpha$-branched galactosylceramide. This was modified slightly for optimal efficacy to produce a compound termed KRN7000, commonly referred to as $\alpha$-GalCer ((298), Figure 1.6). It was not until a few years later that $\alpha$-GalCer was identified as an NKT cell ligand (299), binding to CD1d. The CD1d- $\alpha-G a l C e r$ complex is recognised by both human and mouse NKT cells (300). To date, $\alpha$-GalCer is the best-characterised NKT cell ligand, with proven capacity to stimulate strongly both murine and human NKT cells to produce both IFN $\gamma$ and IL-4 $(299,301)$.

It is unlikely that this antigen recognition system has been conserved to defend us from marine sponges; thus it is assumed that $\alpha$-GalCer is only a mimic of the natural ligand that NKT cells recognise. In support of this, it has been found that glycosphingolipids from the bacteria wall of Sphingomonas were also able to activate NKT cells (302-304). A. mauritianus is known to be colonised by Sphingomonas; therefore, rather than being derived from marine sponges, $\alpha$ GalCer may in fact have originated from bacterial symbionts (274).

\subsubsection{OCH is an analogue of $\alpha$-GalCer}

Many synthetic analogues of $\alpha$-GalCer have been produced to determine structure-function relationships. One of these analogues that is also used in this thesis is $\mathrm{OCH}$. OCH has a 
substantially shorter phytosphingosine chain and unlike $\alpha$-GalCer preferentially induces NKT cells to produce IL-4 ((305-307), Figure 1.6). Biased IL-4 production by NKT cells stimulated with $\mathrm{OCH}$ resulted in the suppression of experimental autoimmune encephalomyelitis in mice (305). OCH is a weaker NKT cell agonist than $\alpha$-GalCer because stimulation of a NKT cell hybridoma with OCH resulted in reduced IL-2 production compared to $\alpha$-GalCer (307). In addition, $\mathrm{OCH}$ stimulation led to reduced proliferation of splenocytes compared with $\alpha$ GalCer stimulation (305). This correlated with the finding that $\mathrm{OCH}$ has a less stable association with CD1d compared with $\alpha$-GalCer. It has been proposed that this less stable binding of OCH leads to the preferential production of IL-4 (306).

\subsubsection{Endogenous NKT cell ligands}

To date, the endogenous ligand for positive selection of NKT cells remains to be identified. It is thought that the self-ligand is likely to be a glycolipid similar to $\alpha$-GalCer. The endogenous glycosphingolipid, isoglobotrihexosylceramide (iGb3) can activate mouse and human NKT cells ((308), Figure 1.6), although iGb3 is a much weaker agonist than $\alpha$-GalCer, requiring 30-100 fold higher concentrations to achieve the same level of NKT cell stimulation. Studies have implicated iGb3 as the natural antigen involved in NKT cell positive selection in the thymus (308), although this is currently highly controversial (286). In addition, humans lack the functional iGb3 synthase found in mouse; thus iGb3 may not be a physiological antigen for human NKT cells. Nevertheless, an alternative synthesis pathway might exist (309). Future studies hope to reveal the natural ligand for NKT cells and this may provide further insights into NKT cell development. 


\section{$\alpha$-GalCer}

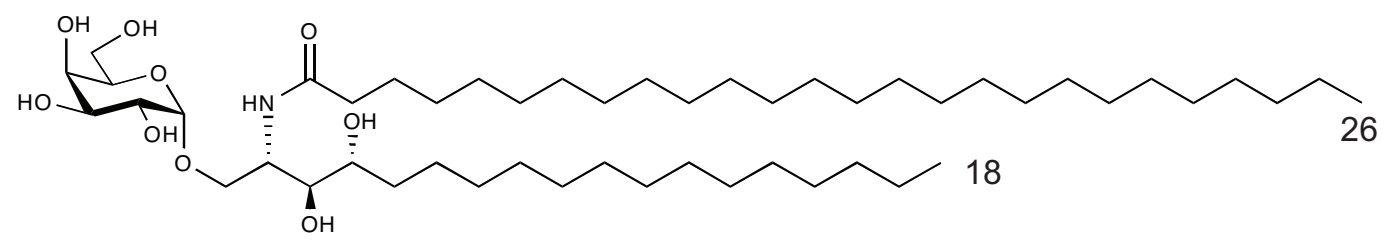

\section{$\mathrm{OCH}$}

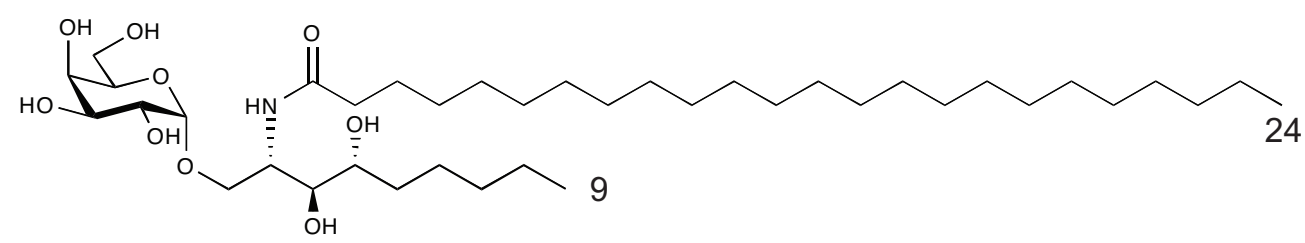

iGb3

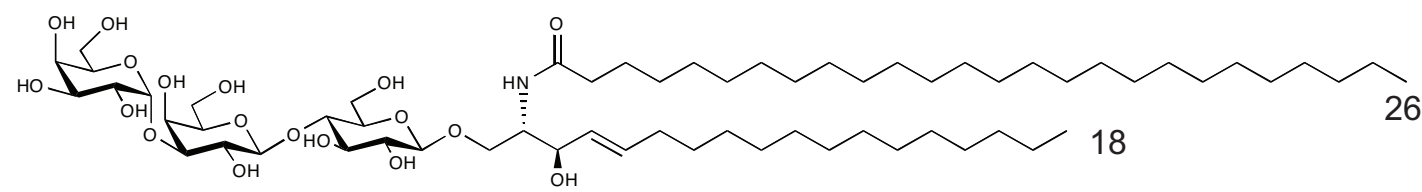

Figure 1.6 Structures of some NKT cell ligands

The strong NKT cell ligand $\alpha$-GalCer is composed of a galactose head group that is linked to a ceramide lipid with an $\alpha$-linkage. The ceramide lipid is made up of an unfunctionalised lipid chain and a shorter phytosphingosine lipid chain. The synthetic analogue $\mathrm{OCH}$ has a truncated phytosphingosine lipid chain. A potential natural ligand for NKT cells is isoglobotrihexosylceramide (iGb3). In contrast to $\alpha$-GalCer and $\mathrm{OCH}, \mathrm{iGb} 3$ has three sugar residues connected to the ceramide lipid via a $\beta$-linkage. The ceramide of iGb3 contains a sphingosine lipid rather than the phytosphingosine found in $\alpha$-GalCer and $\mathrm{OCH}$. The lengths of the lipid chains are indicated.

\subsubsection{Cytolytic functions of NKT cells}

Mouse and human NKT cells have been shown to express perforin $(235,310-313)$ and granzyme B (310). Through the use of either NKT cells from PKO mice, or concanamycin A to block perforin secretion from WT NKT cells, perforin has been shown to be important for lysis of a number of tumour cell lines by NKT cells (312-315). By contrast, in an in vivo 
tumour model, perforin production from NKT cells was not essential for controlling tumour growth (316). These results indicate that in some situations perforin mediated killing by NKT cells could play a role in regulating immune responses. Further in vivo investigations of perforin mediated killing by NKT cells needs to be undertaken to fully elucidate its role.

Upon stimulation, human NKT cells also express the ligands FasL and TRAIL on their cell surface $(152,317,318)$, which can bind their respective receptors to induce cell death. Coculturing of myeloid leukaemia cells with NKT cells led to apoptosis of the leukaemia cells. However, blocking TRAIL with a neutralising antibody almost completely reduced apoptosis in these cells (318). Furthermore, reduced NKT cell cytotoxicity against certain tumour cell lines was observed upon the addition of TRAIL and FasL neutralising antibodies (315), indicating that both TRAIL and FasL are important for NKT cell anti-tumour activity. In addition to killing of tumour cells, FasL expression is also crucial for lysis of DP thymocytes by mouse thymic NKT cells (317). Taken together, these results indicate that NKT cells can directly lyse a number of tumour cells as well as control NKT cell development through the lysis of DP thymocytes. Thus, in addition to the extensive array of cytokines rapidly produced by NKT cells, their cytolytic functions also appear to be valuable in immune responses.

\subsubsection{Immunoregulatory functions of NKT cells}

Following NKT cell stimulation, many other cell types are directly or indirectly activated. The interaction between NKT cells and DC plays a central role in these responses (Figure 1.7). After $\alpha$-GalCer treatment, DC upregulate expression of the co-stimulatory molecules CD40, CD80, CD86 and MHC II $(319,320)$ as well as producing cytokines such as TNF- $\alpha$ and IL-12 (187). Production of IL-12 by DC has been shown to be important for IFN- $\gamma$ production by NKT cells (122). IL-12 in combination with increased production of IFN- $\gamma$ by NKT cells leads to the activation of NK cells, which in turn produce more IFN- $\gamma$ and have lytic functions (321, 322). In addition to IFN- $\gamma$, the production of IL- 2 by NKT cells has also been found to be important for NK cell cytotoxicity (323). It has been proposed that NKT cells themselves do not directly kill tumour cells by cytolytic activity but instead rapidly activate NK cells via IFN- $\gamma$ and IL-2, which then results in NK-mediated cell lysis (321-324). However, this does 
not account for the CD1d-dependent NKT cell killing observed in vitro (323) or the direct cytotoxicity against many tumour cell lines $(312-315,317)$. Thus it is possible that both mechanisms, NKT cell mediated cytotoxicity and cytotoxicity mediated by IFN- $\gamma$-activated NK cells, can occur. Regardless of the cell type mediating the cytotoxicity, NKT cell stimulation enhances cytotoxic responses in vivo.

Activated NKT cells can also influence the behaviour of cells of the adaptive immune response (Figure 1.7). As discussed above, cognate NKT cell-DC interactions result in DC activation, which is important for $\mathrm{T}$ cell activation. Following administration of $\alpha$-GalCer together with the protein antigen ova, ova-specific $\mathrm{CD} 4^{+}$and $\mathrm{CD} 8^{+} \mathrm{T}$ cell responses were elicited $(319,320,325,326)$. The development of ova-specific T cells resulted in protection against ova-expressing tumours $(319,320,325)$. Simultaneous injection of $\alpha$-GalCer and ova protein was required for the development of T cell responses (320), indicating that $\alpha$-GalCer and ova protein may need to be presented by the same DC. Further investigation into NKTDC interactions in vivo demonstrated that TNF- $\alpha$ and IFN- $\gamma$ were important for the upregulation of co-stimulatory molecules on DC and the T cell response to protein antigen (187). Additional studies demonstrated that the CD40-CD40L mediated signals between DC and NKT cell were essential for the interaction to result in T cell immunity $(187,320)$. Thus the NKT cell-DC interaction is important not only for the activation of NK cells but also conventional $\mathrm{T}$ cells which are crucial for certain immune responses such as tumour immunity.

NKT cell activation can also provide help to B cells (Figure 1.7). After $\alpha$-GalCer treatment B cells were shown to upregulate activation markers CD86, CD69 and MHC II, in a CD1d NKT cell-dependent manner, leading to proliferation and antibody secretion (327-332).

Furthermore, B cell receptor internalisation of CD1d-restricted antigens was reported to be effective at stimulating B cell proliferation and the development of early specific antibody responses in vivo $(333,334)$. Additionally, NKT cells were shown to play a role in sustaining specific B cell responses and memory (335). Therefore, through activation of NKT cells both $\mathrm{B}$ and $\mathrm{T}$ cells of the adaptive immune response are affected. Consequently, the DC-NKT cell 
interaction is pivotal to the effector function of the NKT cell and enables the NKT cell to skew the immune response towards different outcomes.

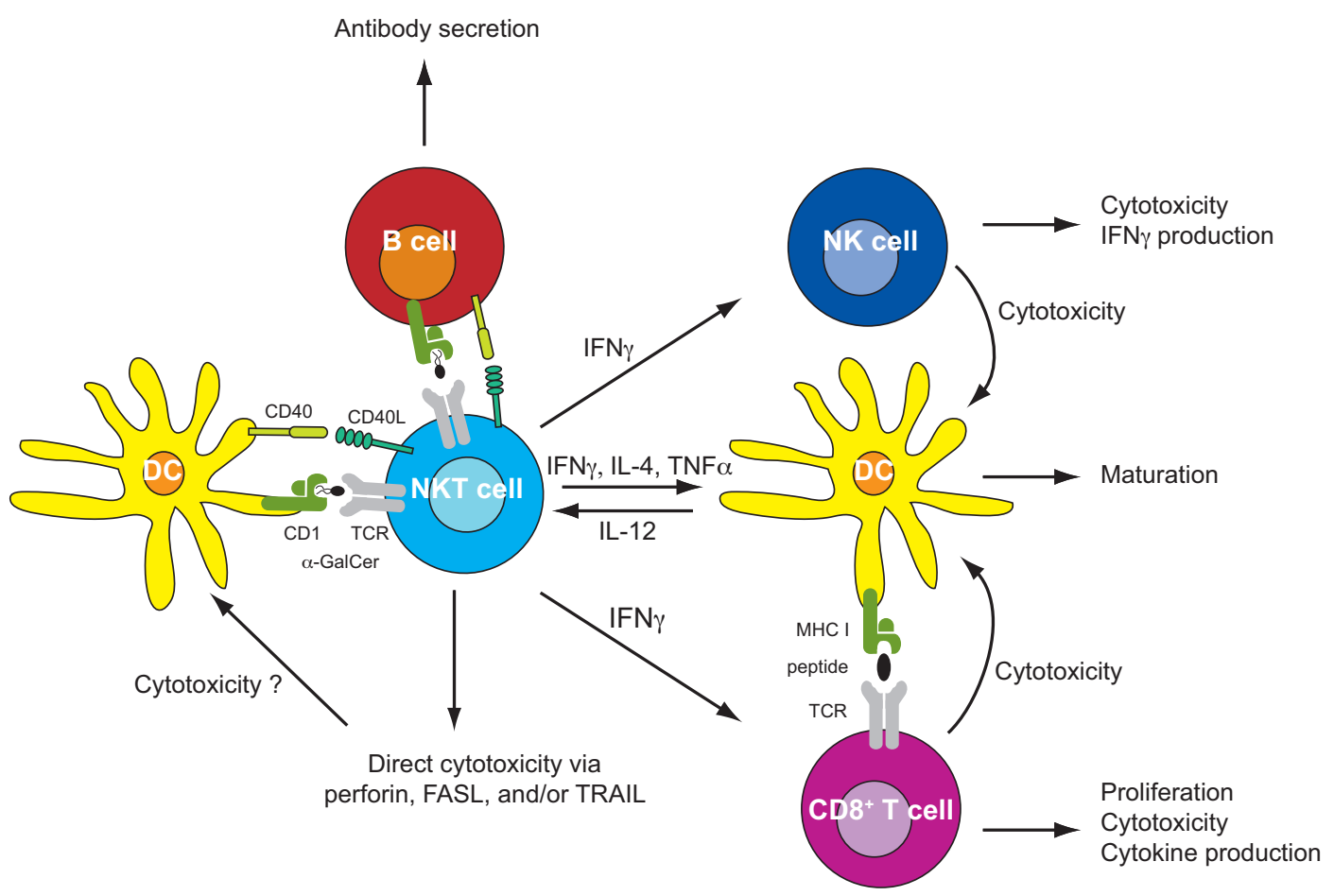

Figure 1.7 NKT cells interact with and activate many different cell types

Recognition of $\alpha$-GalCer presented on CD1d molecule on DC by NKT cells results in the reciprocal activation of both NKT cells and DC. This, in turn, results in the activation of T cells, NK cells and B cells. NK and $\mathrm{CD}^{+} \mathrm{T}$ cells then may have a cytotoxic effect on DC. There is some evidence that NKT cells can also have a direct cytotoxic effect on DC. 


\subsection{DC killing by $\mathrm{CD8}^{+} \mathrm{T}$ cells and NKT cells}

Because of their pivotal role in the immune system, it is important that DC survival and regulation is under tight control. Therefore the mechanisms and cells involved in regulating DC survival are of great interest. This topic has been reviewed in section 1.1.5.

Several authors have proposed the presence of a regulatory feedback network, whereby CTL eliminate DC to control the magnitude of the immune response $(153,155,336)$. In support of this, rapid elimination of antigen-loaded DC was observed after immunisation of CD8 ${ }^{+}$TCR transgenic and immune non-transgenic mice $(169,337)$. Additionally, elimination of antigenloaded DC by effector $\mathrm{CD} 8^{+} \mathrm{T}$ cells was shown to limit the induction of tumour immunity following immunisation of mice with peptide-loaded DC (169), and prevented boosting of existing immune responses $(169,337)$, thus illustrating the importance of DC survival during certain immune responses. Early studies demonstrated a link between perforin and enhanced immune responses. In the absence of perforin, an enhanced $\mathrm{CD} 8^{+} \mathrm{T}$ cell response was observed following infection with L. monocytogenes, Lymphocytic Choriomeningitis Virus (LCMV), or during mixed lymphocyte reactions (338-340). These results suggest that, in the absence of perforin, DC may survive longer and induce better $\mathrm{CD}^{+} \mathrm{T}$ cell responses. More recently, a direct link has been established between CTL mediated killing of DC and expression of perforin by CTL. DC injected into immune PKO mice were not eliminated, in contrast to those injected into immune WT mice (155). This lead to a progressive increase in the number of antigen-specific $\mathrm{CD}^{+} \mathrm{T}$ cells in $\mathrm{PKO}$ mice after repeated immunisation with the antigen-loaded DC (155). Also, during a secondary influenza infection, DC in mediastinal $\mathrm{LN}$ have been found to be killed in a perforin-dependent manner (156). Furthermore, patients with familial hemophagocytic lymphohistiocytosis (FHL), who have mutations in the perforin gene, have uncontrolled T cell activation, resulting in a fatal outcome (341). This uncontrolled T cell activation in FHL patients is thought be to due to increased DC survival since CTL are unable to eliminate DC as they lack the expression of perforin (342). Overall these data suggest that CTL can eliminate DC in a perforin-dependent manner, and that this mechanism may contribute to regulation of immune responses. 
Other studies have endeavoured to determine if effector CTL and memory CD8 ${ }^{+} \mathrm{T}$ cells can both eliminate DC. Recently, in vitro work demonstrated that only CTL kill DC, while memory $\mathrm{CD}^{+} \mathrm{T}$ cells cause DC to enhance IL-12 production and increase the expression of SPI-6 (343). This is in contrast to previously published work by our laboratory where in a memory setting, DC were also eliminated, although with slower kinetics compared to DC elimination by CTL (169). Furthermore, both memory CD8 ${ }^{+} \mathrm{T}$ cells and CTL eliminated DC in vivo even though DC had upregulated SPI-6 (344). Thus, the in vivo evidence points towards the ability of both CTL and memory $\mathrm{CD} 8^{+} \mathrm{T}$ cells to eliminate DC.

Investigations have also been undertaken to determine where CTL eliminate DC. DC elimination by CTL was shown to occur only in peripheral tissues in experiments that altered the injection timing of antigen-loaded DC and specific CTL (155). Sallusto and colleagues, using two-photon microscopy to visualise DC-T cell interactions within the LN, found that after a stable T cell-DC interaction, DC displayed prominent blebbing and fragmentation consistent with the induction of apoptotic cell death (345). The authors therefore proposed that DC elimination by CTL can also occur within the LN. The question still remains as to how significant the DC killing in the LN is compared to that observed outside the LN. Therefore this aspect of DC elimination by CTL remains controversial.

NKT cells also have cytolytic ability and could kill DC in a similar manner to $\mathrm{CD}^{+} \mathrm{T}$ cells. It has been proposed that DC killing is one of the immune regulatory functions of NKT cells, to prevent excessive antigen stimulation $(151,152)$ and regulate the Th1/Th2 balance (310). Human NKT cells can lyse DC in vitro in an $\alpha$-GalCer, CD1d-dependent manner $(150,151$, 310). DC cytolysis by NKT cells was shown to be abrogated by calcium chelation. As the perforin mediated pathway is calcium dependent, this would suggest that perforin is involved in the elimination of DC by NKT cells (310). Additionally, treatment with a neutralising antibody against FasL reduced apoptosis of DC cultured with human NKT cells, indicating that the FasL pathway could also be a mechanism that NKT cells utilise to kill DC (152). Recently, a study found that human DN NKT cells cultured with DC induced increased death of the DC compared to $\mathrm{CD}^{+} \mathrm{NKT}$ cells (296). However, the mechanism of the DC death by the NKT cells was not investigated, but was consistent with the differential expression of 
perforin in the NKT cell subsets (235). Therefore, these in vitro studies suggest that in addition to CTL, NKT cells could also regulate immune responses by eliminating DC.

\subsection{Aims}

Regulation of DC survival and activity is critical to the outcome of immune responses. The evidence presented in recent literature supports a regulatory role for CTL in DC survival, which occurs by a perforin-dependent mechanism. In addition, there is evidence to suggest that NKT cells have an immunoregulatory role in eliminating DC. At the start of this project, the strongest evidence for DC elimination by CTL had been generated in a model using BMDC loaded with peptide antigen and TCR transgenic $\mathrm{CD} 8^{+} \mathrm{T}$ cells (155). No in vivo data had been generated at that time, to demonstrate a direct role of NKT cells in regulating DC survival. Therefore, significant questions yet to be answered were whether CTL eliminate DC in a perforin-dependent manner during a physiologically relevant immune response, and whether NKT cell eliminate DC in an in vivo setting. The aim of this thesis was to answer these questions.

\subsubsection{Specific Aims}

- The first aim of this thesis was to use the murine influenza A virus model to determine whether the virus specific $\mathrm{CD}^{+} \mathrm{T}$ cell response was enhanced in $\mathrm{PKO}$ mice compared to $\mathrm{C} 57 \mathrm{BL} / 6$ mice, and to correlate the magnitude of the $\mathrm{CD} 8^{+} \mathrm{T}$ cell response to $\mathrm{DC}$ survival in the two mouse strains. More DC survival was expected to correlate with increased $\mathrm{CD}^{+} \mathrm{T}$ cell response.

- The second aim was to use the potent NKT cell ligand $\alpha$-GalCer and the model protein antigen ova to determine whether either ova-specific CTL or NKT cells regulated DC survival in a perforin-dependent manner. 
- During investigations into the second aim a decrease in the splenic $\mathrm{CD} 8^{+} \mathrm{DC}$ subset was identified during treatment with $\alpha$-GalCer. The third and final aim was to further characterise the kinetics of this decrease, and determine the mechanism by which the splenic $\mathrm{CD}^{+} \mathrm{DC}$ were lost after treatment with the NKT cell ligand $\alpha-\mathrm{GalCer}$. 
Chapter 2:

Materials and Methods 


\subsection{Materials}

\subsubsection{Labware}

A list of plasticware and suppliers is given below.

\begin{tabular}{|c|c|}
\hline Product & Supplier/Distributor \\
\hline $\begin{array}{l}\text { ABI Prism }{ }^{\circledR} 96 \text { well optical reaction (PCR) } \\
\text { plates }\end{array}$ & $\begin{array}{l}\text { Applied Biosystems, Foster City, CA, } \\
\text { USA }\end{array}$ \\
\hline $\begin{array}{l}\text { Acrodisc }{ }^{\circledR} 32 \mathrm{~mm} \text { syringe filters with } 0.2 \mu \mathrm{m} \\
\text { Supor }^{\circledR} \text { membrane }\end{array}$ & PALL LifeSciences, Cornwall, U.K \\
\hline Axygen Micro Tubes $1.7 \mathrm{~mL}$ & $\begin{array}{l}\text { Axygen Scientific Inc., Union City, } \\
\text { CA, USA }\end{array}$ \\
\hline $\begin{array}{l}\text { BD } 10 \mathrm{~mL} \text { syringes \& BD } 1 \mathrm{~mL} \text { tuberculin } \\
\text { syringes }\end{array}$ & BD BioSciences, Bedford, MA, USA \\
\hline BD Insyte I.V. Catheter & BD BioSciences \\
\hline $\begin{array}{l}\text { BD Falon }{ }^{\mathrm{TM}} \text { sterile nylon cell strainer }(70 \\
\mu \mathrm{M})\end{array}$ & BD BioSciences \\
\hline $\begin{array}{l}\text { BD Falcon }{ }^{\mathrm{TM}} \text { Polystyrene tissue culture } \\
\text { plates: } 6 \text { well plates, Multiwell plates \& } \\
\text { Microtest }^{\mathrm{TM}} \text { U-bottom } 96 \text { well plates }\end{array}$ & BD BioSciences \\
\hline $\begin{array}{l}\text { BD Falcon }{ }^{\mathrm{TM}} \text { Polystyrene tissue culture } \\
\text { flasks: } 200 \mathrm{~mL} \& 600 \mathrm{~mL}\end{array}$ & BD BioSciences \\
\hline $\begin{array}{l}\text { BD Falcon }{ }^{\mathrm{TM}} \text { Polystyrene conical tubes: Blue } \\
\text { Max } 50 \mathrm{~mL} \text { \& Blue Max Jr } 15 \mathrm{~mL}\end{array}$ & BD BioSciences \\
\hline BD Falcon $^{\mathrm{TM}}$ Polystyrene serological pipettes & BD BioSciences \\
\hline Nunc-Immuno $^{\mathrm{TM}}$ Plate, MaxiSorp ${ }^{\mathrm{TM}}$ Surface & Nunc Brand Products, Denmark \\
\hline Nylon Gauze $(70 \mu \mathrm{m})$ & $\begin{array}{l}\text { NZ Filter Specialists Ltd., Onehunga, } \\
\text { Auckland, NZ }\end{array}$ \\
\hline $\begin{array}{l}\text { PrecisionGlide }{ }^{\mathrm{TM}} \text { Needles }(18,21 \& 25 \\
\text { gauge) }\end{array}$ & BD BioSciences \\
\hline $\begin{array}{l}\text { RNAse free Pestle Disp with } 1.5 \mathrm{~mL} \\
\text { microtube }\end{array}$ & $\begin{array}{l}\text { Kontes glass company, Vineland New } \\
\text { Jersey, USA }\end{array}$ \\
\hline TitreTubes ${ }^{\circledR}$ Micro Tubes & BioRad, Hercules, CA, USA \\
\hline Transfer pipettes $(1 \mathrm{~mL})$ & Samco Scientific, Mexico \\
\hline Ultra-Fine $^{\mathrm{TM}}$ needle Insulin syringes (29 & BD BioSciences \\
\hline
\end{tabular}


gauge)

\subsubsection{Reagents and Buffers}

\subsubsection{General Buffers and Solutions}

Ammonium Chloride Tris (ACT) Lysis buffer

Immediately before use, 9 parts $\mathrm{NH}_{4} \mathrm{Cl}$ (Sigma, St Louis, MO, USA), $\mathrm{pH} 7.4$, was combined with 1 part Tris- $\mathrm{HCl}$ (Merck, Darmstadt, Germany), $\mathrm{pH} 7.4$, to give a final concentration of $0.144 \mathrm{M} \mathrm{NH}_{4} \mathrm{Cl}$ and $0.017 \mathrm{M} \mathrm{HCl}$.

\section{Alsever's Solution}

Dextrose (BDH Laboratory supplies, Poole, England), $\mathrm{NaCl}$ (BDH Laboratory supplies), and Sodium citrate. $2 \mathrm{H}_{2} \mathrm{O}$ (BDH Laboratory supplies) were dissolved in $1 \mathrm{~L} \mathrm{dH}_{2} \mathrm{O}$ to give final concentration of $20.5 \mathrm{mg} / \mathrm{mL}$ dextrose, $4.2 \mathrm{mg} / \mathrm{mL} \mathrm{NaCl}$ and $8.0 \mathrm{mg} / \mathrm{mL}$ sodium citrate. $2 \mathrm{H}_{2} \mathrm{O}$. The $\mathrm{pH}$ was adjusted to 6.1 with $1 \mathrm{M}$ citric acid (BDH Laboratory supplies) and the solution stored at room temperature (RT).

\section{Anaesthetic}

A $10 \mathrm{x}$ concentration of anaesthetic was made by combining $10 \mathrm{~mL}$ ketamine $(100 \mathrm{mg} / \mathrm{mL}$, Phoenix, Auckland, New Zealand) with $1.5 \mathrm{~mL}$ of xylazine $(20 \mathrm{mg} / \mathrm{mL}$, Phoenix) and stored in the dark at RT. Immediately before use the $10 \mathrm{X}$ stock solution was diluted to a $1 \mathrm{X}$ solution using sterile phosphate buffered saline (PBS, GIBCO, Invitrogen, Auckland, NZ).

\section{Bio-Plex ${ }^{T M}$ assay buffer}

Bovine serum albumin (BSA, Henderson, Auckland, NZ), Tween 20 (Sigma), $\mathrm{NaN}_{3}$ (Sigma), and ethylenediaminetetraacetic acid (EDTA, Sigma) were added to 1 L PBS, to give a final concentration of $0.1 \%$ BSA, $0.05 \%$ Tween $20,0.005 \%$ (w/vol) $\mathrm{NaN}_{3}$, and 2.5 mM EDTA. The buffer was stored at $4{ }^{\circ} \mathrm{C}$. 
Bovine Serum Albumin (BSA)

BSA with low endotoxin content $(\leq 1 \mathrm{EU} / \mathrm{mg}$ ) and no IgG was purchased from ICPbio Ltd. (Henderson, Auckland, NZ) in powder form and stored at $4{ }^{\circ} \mathrm{C}$.

BSA was also purchased from Sigma in powder form and stored at $4{ }^{\circ} \mathrm{C}$.

5-(and-6)-carboxyfluorescein diacetate, succinimidyl ester (CFSE)

CFSE was purchased as a powder from Molecular Probes (Eugene, OR, USA) and dissolved in dimethyl sulfoxide (DMSO, Sigma) to a $10 \mathrm{mM}$ solution and stored at $-20{ }^{\circ} \mathrm{C}$

Cell Tracker Orange (5-(and-6)-(((4-chloromethyl)benzoyl)amino)tetramethylrhodamine) (CTO)

CTO was purchased as a powder from Molecular Probes (Eugene, OR, USA) and dissolved in DMSO (Sigma) to a $10 \mathrm{mM}$ solution and stored at $-20^{\circ} \mathrm{C}$.

\section{Collagenase II}

Collagenase II was purchased from Invitrogen. The lyophilised powder was stored at $4{ }^{\circ} \mathrm{C}$ and made up fresh in IMDM to a concentration of $2.4 \mathrm{mg} / \mathrm{mL}$ before use.

DEPC/RNAse free $\mathrm{dH}_{2} \mathrm{O}$

DEPC/RNAse free $\mathrm{dH}_{2} \mathrm{O}$ was purchased from Ambion (Foster city, CA, USA) and stored at RT.

\section{DNase I}

DNase I was purchased from Roche (Mannheim, Germany). The lyophilised powder was dissolved to a concentration of $10 \mathrm{mg} / \mathrm{mL}$ in IMDM and stored at $-20{ }^{\circ} \mathrm{C}$. 
Foetal Calf Serum (FCS)

Mycoplasma and virus screened, and performance-tested FCS that contained less than 0.5 $\mathrm{EU} / \mathrm{mL}$ endotoxin, was purchased from Sigma (batch number $6 \mathrm{C} 0413$ ) and stored at $-20{ }^{\circ} \mathrm{C}$.

Fluorescence Activated Cell Sorting (FACS) Buffer

EDTA (Sigma), $\mathrm{NaN}_{3}$ (Sigma) and FCS were added to $1 \mathrm{~L}$ PBS, to give final concentrations of $10 \mathrm{mM}$ EDTA, $0.01 \%$ (w/vol) $\mathrm{NaN}_{3}$ and $2 \%$ FCS. The buffer was stored at $4{ }^{\circ} \mathrm{C}$.

Geneticin $\mathbb{R}(G 418)$

The selective antibiotic Geneticin ${ }^{\circledR}(\mathrm{G} 418, \mathrm{GIBCO})$ was made into aliquots and stored at -20 ${ }^{\circ} \mathrm{C}$.

Iscove's Modified Dulbecco's Medium (IMDM)

IMDM supplemented with GlutaMAX ${ }^{\mathrm{TM}}, 25 \mathrm{mM}$ HEPES buffer plus $3.024 \mathrm{mg} / \mathrm{L} \mathrm{NaHCO}_{3}$ from GIBCO was purchased from Invitrogen.

Complete IMDM (cIMDM)

cIMDM was made by adding Penicillin-Streptomycin (GIBCO, Invitrogen), 2-ME and FCS to $0.5 \mathrm{~L}$ IMDM to give final concentrations of $100 \mathrm{U} / \mathrm{mL}$ Penicillin, $100 \mu \mathrm{g} / \mathrm{mL}$ Streptomycin, $55 \mu \mathrm{M} 2-\mathrm{ME}$ and $5 \%$ FCS. cIMDM was stored for up to 1 month at $4{ }^{\circ} \mathrm{C}$.

\section{Liberase CI}

Liberase CI was purchased from Roche. The lyophilised powder was diluted in injectable water (DEMO S.A Pharmaceutical Industry, Athens, Greece) to a final concentration of 16.4 
$\mathrm{mg} / \mathrm{mL}$. Aliquots were stored at $-20^{\circ} \mathrm{C}$ and further diluted in IMDM just before use to a working concentration of $0.41 \mathrm{mg} / \mathrm{mL}$.

Lipopolysaccharide (LPS)

LPS from Escherichia coli, serotype 0111:B4, was purchased from Sigma. The lyophilised powder was dissolved to a concentration of $1 \mathrm{mg} / \mathrm{mL}$ in IMDM and stored at $4{ }^{\circ} \mathrm{C}$.

2 Mercaptoethanol (2 ME)

$2 \mathrm{ME}$ solution in PBS was purchased from Sigma as a $55 \mathrm{mM}(1000 \mathrm{x})$ and stored at $4{ }^{\circ} \mathrm{C}$.

Phosphate Buffered Saline (PBS) pH 7.2

One $47.8 \mathrm{~g}$ bottle of Dulbecco's PBS without $\mathrm{CaCl}_{2}$ and $\mathrm{MgCl}_{2}$ (Sigma Aldrich, Auckland,

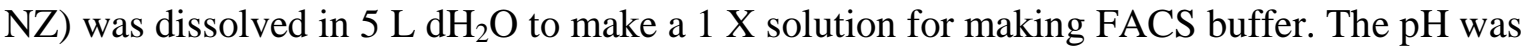
adjusted to 7.4 and the solution was stored at $4{ }^{\circ} \mathrm{C}$.

PBS used at other times was purchased from GIBCO in $500 \mathrm{~mL}$ bottles. Bottles were stored at RT until opened. Once opened bottles were stored at $4{ }^{\circ} \mathrm{C}$.

Trypsin/EDTA (Ethylenediaminetetraacetic acid)

Trypsin/EDTA solution containing $0.25 \%$ Trypsin and 1 mM EDTA in Hanks Balanced Salt Solution (GIBCO) were stored at $-20{ }^{\circ} \mathrm{C}$. Aliquots were thawed as required and kept at $4{ }^{\circ} \mathrm{C}$.

\section{Würzburger Buffer}

EDTA (Sigma), DNAse I and FCS were added to 1 L PBS, to give final concentrations of 5 mM EDTA, $0.02 \mathrm{mg} / \mathrm{mL}$ DNAse I and $1 \%$ FCS. Buffer was stored at $4{ }^{\circ} \mathrm{C}$. 


\subsubsection{Solutions for enzyme linked immunosorbent assay (ELISA)}

\section{ELISA Buffer}

BSA (Sigma), $5 \mathrm{~g}$ and $0.1 \mathrm{~g}$ of Thimerosal (Sigma) was dissolved in $1 \mathrm{~L}$ of $1 \mathrm{X}$ wash buffer. The $\mathrm{pH}$ was adjusted to 7.4 and the buffer stored at $4{ }^{\circ} \mathrm{C}$ for no longer than one month.

\section{ELISA Blocking Buffer}

BSA (Sigma), $5 \mathrm{~g}$ was added to $250 \mathrm{~mL}$ of PBS, to give a final concentration of $5 \% \mathrm{BSA}$. The $\mathrm{pH}$ was adjusted to 7.4 and the buffer stored at $4{ }^{\circ} \mathrm{C}$ for no longer than 3 weeks.

\section{ELISA Coating Buffer}

$\mathrm{Na}_{2} \mathrm{CO}_{3}$ (BDH Laboratory Supplies), $1.59 \mathrm{~g}$ and $2.93 \mathrm{~g}$ of $\mathrm{NaHCO}_{3}$ were dissolved in $1 \mathrm{~L}$ of $\mathrm{dH}_{2} \mathrm{O}$ and the $\mathrm{pH}$ adjusted to 9.6. The solution was stored at $4{ }^{\circ} \mathrm{C}$ for no longer than 3 weeks.

\section{ELISA Stopping Solution (2 mM Sodium Azide solution)}

$\mathrm{NaN}_{3}$ (Sigma) was dissolve in $200 \mathrm{~mL}$ of $\mathrm{dH}_{2} \mathrm{O}$, giving a final concentration of $2 \mathrm{mM}$. The solution was stored at RT.

\section{ELISA Substrate Buffer}

Citric acid (BDH Laboratory Supplies), $2.82 \mathrm{~g}$ and $2.92 \mathrm{~g}$ of $\mathrm{NaH}_{2} \mathrm{PO}_{4}$ were dissolved in 500 $\mathrm{mL}$ of $\mathrm{dH}_{2} \mathrm{O}$ and $\mathrm{pH}$ adjusted to 4.2 . The solution was stored at $4{ }^{\circ} \mathrm{C}$. 
ELISA Substrate Solution (ABTS-2,2'-azino-di (3-ethylbenzthiazolinesulfonic acid) in substrate buffer $p H 4.2)$

ABTS (Sigma), $0.0548 \mathrm{~g}$ was dissolved in $100 \mathrm{~mL}$ Substrate Buffer. The solution was stored in aliquots at $-20{ }^{\circ} \mathrm{C}$ protected from light. Immediately before use, $1 \mu \mathrm{l}$ of $30 \% \mathrm{H}_{2} \mathrm{O}_{2}(\mathrm{BDH}$ Laboratory Supplies) was added per $1 \mathrm{~mL}$ of substrate solution.

\section{ELISA Wash Buffer}

$\mathrm{NaCl}$ (BDH Laboratory Supplies) $32 \mathrm{~g}, 0.8 \mathrm{~g}$ of $\mathrm{KH}_{2} \mathrm{PO}_{4}$ (BDH Laboratory Supplies), $4.6 \mathrm{~g}$ of $\mathrm{Na}_{2} \mathrm{HPO}_{4}$ (anhydrous) (BDH Laboratory Supplies), $0.8 \mathrm{~g}$ of $\mathrm{KCl}$ (BDH Laboratory Supplies) and $2 \mathrm{~mL}$ of Tween 20 (Sigma Aldrich, St Louis, MO, USA) were dissolved in $400 \mathrm{~mL}$ of $\mathrm{dH}_{2} \mathrm{O}$ to make a $10 \mathrm{X}$ solution. The $\mathrm{pH}$ was adjusted to 7.4 and stored at $4{ }^{\circ} \mathrm{C}$.

A $1 \mathrm{X}$ solution was made by using 1 part of $10 \mathrm{X}$ solution and 9 parts $\mathrm{dH}_{2} \mathrm{O}$ and stored at $4{ }^{\circ} \mathrm{C}$.

\subsubsection{Cytokines}

\section{Granulocyte-macrophage colony stimulating factor (GM-CSF)}

Recombinant murine GM-CSF was produced using stationary phase cultures of the murine X63 cell line, transfected to secrete the full length murine GM-CSF protein (kindly provided by Dr Antonius Rolink, Basel Institute for Immunology, Basel, Switzerland).

\section{Interleukin $4(I L-4)$}

Recombinant murine IL-4 was produced using stationary phase cultures of a Chinese Hamster Ovary cell line (CHO), transfected to secrete the full length murine IL-4 protein (346).

To collect cytokines from producing cell lines, adherent cells were grown for approximately 2 weeks in cIMDM and culture supernatants were harvested and filtered through a $0.2 \mu \mathrm{M}$ 
serum filter and stored at $-80{ }^{\circ} \mathrm{C}$. Culture supernatants were titrated and an optimal dose used for the growth of bone marrow derived DC (BMDC).

\subsubsection{Glycolipids}

The $\alpha$-Galactosylceramide ( $\alpha$-GalCer) for loading of CD1d tetramer was obtained from Alexis (Sapphire Bioscience, Redfern, Australia) and used as described in section 2.2.5.1. In all remaining experiments, the NKT cell ligands $\alpha$-GalCer and OCH were kindly provided by Gavin Painter (IRL, Lower Hutt, NZ) and were manufactured as described in Lee et al (347). 


\subsubsection{Antibodies, pentamers/multimers and secondary step reagents}

Antibodies used for flow cytometry

\begin{tabular}{|c|c|c|c|}
\hline Specificity & Clone/Isotype & Fluorophore & Source \\
\hline $\begin{array}{l}\text { Fc } \gamma \mathrm{RII} / \mathrm{III} \\
(\mathrm{CD} 32 / \mathrm{CD} 16)\end{array}$ & $2.4 \mathrm{G} 2$ & - & $\begin{array}{l}\text { Purified from B cell hybridoma cell } \\
\text { line }\end{array}$ \\
\hline $\mathrm{B} 220(\mathrm{CD} 45 \mathrm{R})$ & $6 \mathrm{~B} 2$ & PerCp & $\begin{array}{l}\text { BD Pharmingen (San Diego, CA, } \\
\text { USA) }\end{array}$ \\
\hline CD1d & 1B1 & $\mathrm{PE}$ & eBioscience (San Diego, CA, USA) \\
\hline CD3ع & $145-2 \mathrm{C} 11$ & PE-Cy7 & eBioscience \\
\hline CD4 & GK1.5 & Pacific Blue & $\begin{array}{l}\text { Purified from B cell hybridoma cell } \\
\text { line }\end{array}$ \\
\hline \multirow[t]{2}{*}{ CD8 } & $53-6.7$ & FITC & BD Pharmingen \\
\hline & 2.43 & FITC & $\begin{array}{l}\text { Purified from B cell hybridoma cell } \\
\text { line }\end{array}$ \\
\hline CD11b & M1/70 & $\mathrm{PE}$ & BD Pharmingen \\
\hline \multirow[t]{2}{*}{ CD11c } & N418 & $\begin{array}{l}\text { APCy } \\
\text { Alexa } 647\end{array}$ & $\begin{array}{l}\text { Purified from B cell hybridoma cell } \\
\text { line }\end{array}$ \\
\hline & HL3 & APCy & BD Pharmingen \\
\hline CD24 & M1/69 & $\mathrm{PE}$ & BD Pharmingen \\
\hline CD62L & MEL-14 & APCy & BD Pharmingen \\
\hline \multirow[t]{2}{*}{ CD86/B7-2 } & GL1 & Biotin & $\begin{array}{l}\text { Purified from B cell hybridoma cell } \\
\text { line }\end{array}$ \\
\hline & GL1 & $\mathrm{PE}$ & eBioscience \\
\hline CD45.1 & A20 & $\mathrm{PE}$ & eBioscience \\
\hline CD205/DEC 205 & NLDC 145 & $\begin{array}{l}\text { Biotin } \\
\text { FITC }\end{array}$ & $\begin{array}{l}\text { Purified from B cell hybridoma cell } \\
\text { line }\end{array}$ \\
\hline \multirow[t]{2}{*}{ IFN- $\gamma$} & XMG1.2 & APCy & BD Pharmingen \\
\hline & Rat IgG1a & APCy & BD Pharmingen \\
\hline MHC-II (I-Ab) & M5/114.15 & $\mathrm{PE}$ & BD Pharmingen \\
\hline \multirow[t]{2}{*}{ KLRG1 } & $2 \mathrm{~F} 1$ & APCy & eBioscience \\
\hline & $\begin{array}{l}\text { Golden } \\
\text { Hamster IgG }\end{array}$ & APCy & eBioscience \\
\hline
\end{tabular}


Antibody used for ELISA

\begin{tabular}{|l|l|l|}
\hline Specificity & Clone/Isotype & Source \\
\hline Rat anti-mouse IgG1 & A85-1 & BD Pharmingen \\
\hline
\end{tabular}

Antibody used for neutralisation of TNF- $\alpha$

\begin{tabular}{|l|l|l|}
\hline Specificity & Clone/Isotype & Source \\
\hline TNF- $\alpha$ & TN3-19.12 & $\begin{array}{l}\text { Biolegend (San Diego, CA, } \\
\text { USA) }\end{array}$ \\
\hline
\end{tabular}

Multimers/Pentamers

\begin{tabular}{|l|l|l|l|}
\hline Name & Peptide sequence & Label & Source \\
\hline $\mathrm{NP}_{366-374} \mathrm{H}-2 \mathrm{D}^{\mathrm{b}}$ & ASNENMETM & Unlabelled & $\begin{array}{l}\text { ProImmune Ltd } \\
\text { (Oxford, England) }\end{array}$ \\
\hline PA $_{224-233} \mathrm{H}-2 \mathrm{D}^{\mathrm{b}}$ & SSLENFRAYV & Biotin & ProImmune Ltd \\
\hline Ova $_{257-264} \mathrm{H}-2 \mathrm{~K}^{\mathrm{b}}$ & SIINFEKL & PE & ProImmune Ltd \\
\hline LCMV GP & & & \\
\hline CD141 $\mathrm{H}-2 \mathrm{D}^{\mathrm{b}}$ & KAVYNFATC & Unlabelled & ProImmune Ltd \\
\hline
\end{tabular}

Second step reagents

\begin{tabular}{|l|l|}
\hline Name & Source \\
\hline R-PE Fluorotag & ProImmune Ltd \\
\hline Streptavidin (SA)-FITC, PE, PerCP, APCy & BD Pharmingen \\
\hline SA- Horseradish peroxidase (HRP) & BD Pharmingen \\
\hline
\end{tabular}

Viability dyes used for flow cytometry

\begin{tabular}{|l|l|}
\hline Name & Source \\
\hline 4',6-diamidino-2-phenylindole (DAPI) & Invitrogen \\
\hline Propidium Iodide (PI) & BD Pharmingen \\
\hline
\end{tabular}




\subsubsection{Peptides}

The influenza A nucleoprotein ( $\mathrm{NP}_{366-374}$, ASNENMETM) and influenza A Polymerase acidic protein $\left(\mathrm{PA}_{224-233}\right.$, SSLENFRAYV) peptides were purchased from Mimotopes Pty Ltd. (Clayton, Victoria, Australia).

\subsubsection{Tumour cell line}

The B16.ova tumour cell line was generated by E.Lord and J.G. Frelinger (348). The tumour cell line was derived by transfection of the parental B16.F0 tumour cell line with cDNA encoding chicken ovalbumin.

The tumour cell line was maintained in cIMDM containing $0.5 \mathrm{mg} / \mathrm{mL}$ Geneticin ${ }^{\circledR}$ selective antibiotic. Adherent cells were detached by 1 min incubation at $37^{\circ} \mathrm{C}$ with $2 \mathrm{ml}$ Trypsin/EDTA. Proteolysis was stopped by adding an equal volume of FCS and cells were washed 3 times in IMDM before use.

\subsubsection{Mice}

\subsubsection{Mouse ethics approval}

All experimental procedures were approved by and carried out with the ethical approval of the Victoria University Animal Ethics Committee and performed according the guidelines of Victoria University. The ethics protocol numbers used throughout this thesis were: 2003R23M, 2004R5, 2004R21M, 2006R19, 2007R3M, 2007R17M.

\subsubsection{Mouse maintenance}

All animals were maintained and bred in the Biomedical Research Unit, Malaghan Institute of Medical Research, based at Victoria University, Wellington, New Zealand. 


\subsubsection{Mouse strains}

C57BL/6, interferon gamma knockout (IFN- $\gamma$ KO) (349), and perforin knockout (PKO) mice on a C57BL/6 background (244) were originally purchased from The Jackson Laboratory (Bar Harbour, ME, USA). PKO mice were genotyped and shown to contain the inactivated perforin allele as reported in Kagi et al (244). Furthermore, experiments carried out in the laboratory while this thesis work was being carried out consistently found that immune PKO mice lack the ability to eliminate DC in vivo, which is in contrast to immune C57BL/6 mice that rapidly eliminate DC (unpublished data and (155)).

Mice deficient in CD1 (CD1 KO) on a C57BL/6 background were obtained from CR Wang (Department of Pathology, University of Chicago, IL, USA) and generated as described in Chen et al (350).

Langerin-EGFP knockin mice, on a C57BL/6 background, expressing enhanced green fluorescent protein (EGFP) under the control of the Langerin promoter, as described in Kissenpfennig et al (57), were kindly provided by B. Malissen, Centre d'Immunologie Marseille-Luminy, France.

Mice lacking Fas expression (B6.MRL.Fas ${ }^{\text {lpr }}$ or B6.lpr) were from breeding pairs obtained from The Walter and Eliza Hall Institute of Medical Research, Melbourne, Australia.

OT-IxB6.congenic mice were created onsite by breeding B6.SJL-Ptprc ${ }^{a} P e p 3$ /BoyJARC mice, obtained from the Animal Resources Centre (Canning Vale, WA, Australia), with OT-I mice (351) provided by S. Hook (School of Pharmacy, Dunedin, NZ) with the permission of F.Carbone (Melbourne University, Melbourne, Australia), until homozygous for the transgenic TCR.

All mice strains were maintained by brother x sister mating at the Malaghan Institute of Medical Research. At the commencement of each experiment, mice were aged 6-12 weeks and mice within each experiment were age- and sex-matched. 


\subsection{Methods}

\subsubsection{General cell culture}

All cells were cultured in cIMDM at $37^{\circ} \mathrm{C}$, with $5 \% \mathrm{CO}_{2}$ and $95 \%$ humidity.

\subsubsection{Culture of Dendritic Cells}

\subsubsection{Culture from Bone marrow (BM) precursors}

Mice were first euthanised by asphyxiation in $\mathrm{CO}_{2}$, and the hind limbs were detached at the hip joints and placed into a $10 \mathrm{~mL}$ Falcon tube containing IMDM. The muscle and connective tissue were removed, knee joints were separated and the ends of the femurs and tibias were cut off to expose the BM. The marrow was flushed out into a $50 \mathrm{~mL}$ Falcon tube using a 25 -gauge needle and a $10 \mathrm{~mL}$ syringe filled with IMDM. Clumps of cells were disrupted by vigorous pipetting and the suspension was passed through a $70 \mu \mathrm{m}$ cell strainer. Cells were pelleted by centrifugation at $320 \mathrm{~g}$ for $4 \mathrm{~min}$ and resuspended in cIMDM. Live cells were identified by Trypan blue dye (Trypan Blue stain $0.4 \%$, GIBCO) exclusion and counted using a haemocytometer (Weber Scientific International Ltd, Middix, England). Cells were resuspended in cIMDM at $4 \times 10^{5}$ cells/mL and plated out in 6 well plates. Each well contained $5 \mathrm{ml}$ cIMDM containing 2 × $10^{6}$ DC plus $10 \mathrm{ng} / \mathrm{mL}$ GM-CSF and $20 \mathrm{ng} / \mathrm{mL}$ IL-4 (352). Plates were incubated at $37^{\circ} \mathrm{C}$ for 7 days. Cells were supplemented with nutrients on days 3 and 5 by removing $2 \mathrm{~mL}$ medium from each well and replacing with $2 \mathrm{~mL}$ fresh cIMDM containing of $10 \mathrm{ng} / \mathrm{mL}$ GM-CSF and $20 \mathrm{ng} / \mathrm{mL}$ IL-4.

\subsubsection{Activation of DC}

DC were activated by treating with $100 \mathrm{ng} / \mathrm{mL}$ LPS for the last $20 \mathrm{hr}$ of culture. Non-adherent cells were harvested on day 7 by pipetting up the cell suspension and gently washing over the surface of the plate 2 or 3 times. 


\subsubsection{Loading of DC with $\alpha$-Galactosylceramide ( $\alpha$-GalCer)}

DC were loaded with $\alpha$-GalCer by treating with $100 \mathrm{ng} / \mathrm{mL} \alpha$-GalCer (IRL) for the last $40 \mathrm{hr}$ of culture.

\subsubsection{Infection with influenza A viruses}

\subsubsection{Preparation of influenza A virus stocks}

The A/HKx31 (HKx31) influenza A virus is a laboratory-generated recombinant with the external surface components of A/Aichi/2/34 (H3N2) and the internal components of A/Puerto Rico/8/34 (PR8, H1N1). Virus stocks were grown in allantoic cavities of 10-day-old embryonated chicken eggs and stored as infectious allantoic fluid at $-80{ }^{\circ} \mathrm{C}$. Virus stock was shown to be endotoxin free by Limulus amebocyte lysate assay (Sigma). Virus titre was determined by agglutination of human erythrocytes and is expressed as hemagglutinating units (HAU). Stock HKx31 (10 HAU/1 $\mu \mathrm{L})$ was stored at $-80{ }^{\circ} \mathrm{C}$ in $100 \mu \mathrm{L}$ aliquots. Before use a fresh aliquot was diluted in sterile PBS to a concentration of $1 \mathrm{HAU} / 30 \mu \mathrm{L}$.

Influenza virus PR8 was kindly provided by Dr. Margaret Baird (University of Otago, Dunedin, NZ) and prepared as described in Faulkner et al (353). Stock PR8 Virus (0.86 x10 8 $\mathrm{PFU} / \mathrm{mL}$ ) was stored at $-80^{\circ} \mathrm{C}$ in $1 \mathrm{~mL}$ aliquots. Before use a fresh aliquot was diluted in sterile PBS to a concentration of $5 \times 10^{7} \mathrm{PFU} / \mathrm{mL}$.

Both viral stocks were titrated and an optimal dose was determined by measuring weight loss and assessing the $\mathrm{CD} 8^{+} \mathrm{D}^{\mathrm{b}}{ }_{-\mathrm{NP} 366-374^{+}}{ }^{\mathrm{T}}$ cell response. Optimal viral doses chosen were able to

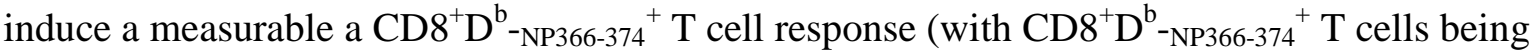
5-10\% of blood $\mathrm{CD}^{+} \mathrm{T}$ cells at day 8-11 after infection) and induced a weight loss that complied with the NZ ethical guidelines (see section 2.1.3.1). 


\subsubsection{Virus infections}

Mice were anaesthetised by receiving an intraperitoneal (i.p.) injection of anaesthetic containing ketamine and xylazine at $100 \mathrm{mg} / \mathrm{kg}$ and $3 \mathrm{mg} / \mathrm{kg}$ body weight respectively followed by an intranasal (i.n.) infection with of $1 \mathrm{HAU}$ of $\mathrm{HKx} 31$ virus diluted in $30 \mu \mathrm{L}$ of sterile PBS. Control mice were injected with $30 \mu \mathrm{L}$ of sterile PBS. This i.n. route of administration was chosen as this the natural route of infection and generates a physiologically relevant immune response. To generate a secondary response, mice were initially primed by i.p. injection with $1 \times 10^{7}$ PFU PR8 virus in $200 \mu \mathrm{L}$ sterile PBS 4 weeks before i.n. challenge with 1 HAU of HKx31 virus. The use of the i.p. route of administration for the PR8 virus was used based on the known virulence of the viral strain and previously published work (354357).

\subsubsection{Analysis of viral titre in lung tissue}

\subsubsection{Collection of lung tissue}

Mice were euthanised with a lethal dose of anaesthetic containing ketamine and xylazine at $400 \mathrm{mg} / \mathrm{kg}$ and $12 \mathrm{mg} / \mathrm{kg}$ body weight respectively and the lung cavity exposed. Lungs were harvested from mice at different time points after an i.n. infection of $\mathrm{HK} \times 31$ and placed into a 1.5 mL RNAse free microtube (Kontes glass company, Vineland New Jersey, USA) containing $500 \mu \mathrm{L}$ of TRIzol ${ }^{\circledR}$ reagent (Invitrogen). Samples were stored at $-80{ }^{\circ} \mathrm{C}$.

\subsubsection{RNA extraction}

Lung tissue was thawed and homogenised using an RNAse free pestle (Kontes glass company). Once the tissue was homogenised $500 \mu \mathrm{L}$ of TRIzol ${ }^{\circledR}$ reagent was added followed by centrifugation at $12000 \mathrm{~g}$ for $10 \mathrm{~min}$ at $4{ }^{\circ} \mathrm{C}$. The supernatant was collected and transferred to a clean $1.7 \mathrm{~mL}$ microtube (Axygen Scientific Inc., Union City, CA, USA) and left at RT for $5 \mathrm{~min}$. Next, $200 \mu \mathrm{L}$ chloroform was added followed by vigorous shaking for $15 \mathrm{sec}$, then left to incubate at RT for 2-3 min. After incubation, samples were centrifuged at $12000 \mathrm{~g}$ for 15 
min at $4{ }^{\circ} \mathrm{C}$ and the colourless aqueous phase was transferred to a new microtube. To precipitate the RNA, $500 \mu \mathrm{L}$ of isopropanol was added to the aqueous phase, mixed and incubated at RT for $10 \mathrm{~min}$ followed by centrifugation at $12000 \mathrm{~g}$ for $10 \mathrm{~min}$. The supernatant was removed and the pellet washed twice in $1 \mathrm{~mL}$ of $75 \%$ ethanol and centrifuged at $7500 \mathrm{~g}$ for $5 \mathrm{~min}$. Ethanol was tipped out, and the pellet was left to air dry and resuspended in $20 \mu \mathrm{L}$ DEPC/RNAse free $\mathrm{dH}_{2} \mathrm{O}$. Contaminating DNA was removed using the DNA-free kit (Ambion, Foster City, CA, USA).

\subsubsection{Quantification of RNA}

RNA concentration was calculated according to the formula: RNA $(\mathrm{mg} / \mathrm{mL})=$ optical density $(\mathrm{OD})$ at $260 \mathrm{~nm}$ x dilution factor $\mathrm{x} 40$

\subsubsection{Generation of cDNA}

cDNA was synthesized from $5 \mu \mathrm{g}$ RNA using the SuperScript ${ }^{\mathrm{TM}}$ III First-Strand Synthesis System for RT-PCR kit and random hexamer primers (Invitrogen) following the manufacturers instructions.

\subsubsection{Real time PCR (RT-PCR)}

Primers as described in Spackman et al (358) and 18S control primers were obtained from Sigma GenoSys (Sigma).

Influenza A virus forward, AGA TGA GTC TTC TAA CCG AGG TCG, Influenza A virus reverse, TGC AAA AAC ATC TTC AAG TCT CTG, 18S forward, GTA ACC CGT TGA ACC CCA TT and 18S reverse, CCA TCC AAT CGG TAG TAG CG. For amplification reactions, cDNA, primers and SYBR Green PCR MasterMix (Applied Biosystems, Foster City, CA) were mixed and run on an ABI prism 7700 Sequence Detection System (Applied Biosystems) for $40 \mathrm{cycles}$ of $15 \mathrm{sec}$ at $95^{\circ} \mathrm{C}$ followed by $1 \mathrm{~min}$ at $62^{\circ} \mathrm{C}$. The amplified products were homogeneous by $\mathrm{Tm}$. For each sample the number of cycles required to reach a pre-determined $\Delta \mathrm{Rn}$ was calculated, and the expression of Influenza A virus in treated mice 
(Exp) relative to $\mathrm{C} 57 \mathrm{~L} / 6$ mice D8 treated control mice (Cont) was calculated as $2^{-\Delta \Delta \mathrm{Ct}}$, where $\Delta \Delta \mathrm{Ct}=\left(\right.$ No. cycles for influenza $\mathrm{A}$ virus $\mathrm{Exp}_{\mathrm{Ep}}-$ No. cycles for $\left.18 \mathrm{~S}_{\mathrm{Exp}}\right)-($ No. cycles for Influenza A virus ${ }_{\text {Cont }}-$ No. cycles for $\left.18 \mathrm{~S}_{\text {Cont }}\right)$.

\subsubsection{Treatment with glycolipids and ova protein}

\subsubsection{Preparation of glycolipids}

NKT cell ligands $\alpha$-GalCer and OCH (IRL) were resuspended in a 10:10:3

methanol:chloroform:water solution and further diluted to a concentration of $200 \mu \mathrm{g} / \mathrm{mL}$ in PBS containing $0.5 \%$ Tween 20 (Sigma). To ensure the solution was completely dissolved it was incubated at $80{ }^{\circ} \mathrm{C}$ for 5 min followed by sonication (Ultrasonic cleaner, Unisonics PTY LTD, Sydney, Australia) for 5 min, then repeated 3 times. A 1 X solution was prepared by diluting the $200 \mu \mathrm{g} / \mathrm{mL}$ stock solution in PBS to a final concentration of $2 \mu \mathrm{g} / \mathrm{mL}$ and stored at $4{ }^{\circ} \mathrm{C}$.

$\alpha$-GalCer from Alexis (Sapphire Bioscience, Redfern, Australia) was diluted to a concentration of $200 \mu \mathrm{g} / \mathrm{mL}$ in PBS containing $0.5 \%$ Tween 20 (Sigma). To ensure the solution was completely dissolved it was incubated at $80{ }^{\circ} \mathrm{C}$ for $5 \mathrm{~min}$ followed by sonication for $5 \mathrm{~min}$, then this was repeated 3 times. The solution was then stored at $4{ }^{\circ} \mathrm{C}$ overnight before CD1d tetramer loading the following day (see section 2.2.7.4).

\subsubsection{Preparation of chicken ovalbumin (ova) protein}

Chicken ova, kindly provided by Thomas Moran (Department of Microbiology, Mount Sinai School of Medicine, New York), was isolated endotoxin-free as described in Brimnes et al (359). The protein was diluted to a final concentration of $60 \mathrm{mg} / \mathrm{mL}$ at stored at $-80{ }^{\circ} \mathrm{C}$. Prior to use the $60 \mathrm{mg} / \mathrm{mL}$ stock solution was further diluted in PBS to the desired concentration. 


\subsubsection{Injection of glycolipids and ova protein}

Prior to treatment the glycolipid solution was sonicated to ensure no particles were present.

When glycolipids were administered in combination with ova protein $1 \mathrm{~mL}$ insulin syringes were layered with $100 \mu \mathrm{L}$ of glycolipid and ova protein to ensure simultaneous injection of both glycolipid and protein. Mice were immunised intravenously (i.v.) with $200 \mathrm{ng}$ of glycolipid unless otherwise stated and $200 \mu \mathrm{g}$ of ova protein in a total volume of $200 \mu \mathrm{L}$. Single administration of either glycolipid or ova protein was injected in a $100 \mu \mathrm{L}$ volume. To boost the ova-specific response and create a secondary $\mathrm{T}$ cell response some mice received an i.v. boost of either $200 \mu \mathrm{g}$ or $2 \mathrm{mg}$ ova protein in a $100 \mu \mathrm{L}$ volume 30 days after the initial treatment of $\alpha$-GalCer and ova protein.

\subsubsection{Tissue Preparation}

\subsubsection{Bronchoalveolar lavage (BAL)}

Mice were euthanised by a lethal dose of anaesthetic as described in section 2.2.4.1 and the trachea exposed. A small incision was made into the trachea and the catheter tube inserted. 1 $\mathrm{mL}$ of sterile PBS was washed through the trachea and lung 3 times. The harvested BAL fluid was collected into FACS tubes and stored on ice. This was repeated 3 times until approximately $3 \mathrm{~mL}$ of BAL fluid was collected in total from each mouse. Cells were then centrifuged at $320 \mathrm{~g}$ for $4 \mathrm{~min}$ and pellets resuspended the appropriate buffer and stored at $4^{\circ} \mathrm{C}$. Before use samples were counted as described in section 2.2.6.6.

\subsubsection{Blood}

Mice were warmed for approximately 5 min under a heat lamp before placing them into restrainers. Once securely restrained, blood was collected by either gently cutting across the lateral tail vein or by tipping the end of the tail with a sterile scalpel blade. Approximately 10 drops of blood was collected into $500 \mu \mathrm{L}$ of Alsever's Solution in a $1.7 \mathrm{~mL}$ microtube. 
Pressure was applied to the cut site and, once bleeding had ceased, mice were returned to their cages.

The blood samples were stored on ice until ready for processing. Samples were centrifuged at $600 \mathrm{~g}$ for $2 \mathrm{~min}$ and the supernatant was removed by aspiration. The pellet was resuspended in $1 \mathrm{~mL}$ ACT buffer and incubated at $37^{\circ} \mathrm{C}$ for 5 min to lyse red blood cells. Samples were centrifuged at $600 \mathrm{~g}$ for $2 \mathrm{~min}$, the supernatant was removed by vacuum and the pellets resuspended in FACS buffer. This was repeated to ensure all trace of ACT buffer was removed. Samples were then resuspended in the appropriate buffer and stored at $4{ }^{\circ} \mathrm{C}$.

\subsubsection{Lymph Nodes (LN)}

Mesenteric lymph nodes (MesLN) were harvested from mice euthanised by $\mathrm{CO}_{2}$ asphyxiation, whereas mediastinal lymph nodes (MLN) were harvested from mice euthanised by a lethal dose of anaesthetic as described in section 2.2.4.1. Harvested LN were placed into cIMDM and stored on ice until they could be processed.

LN were disrupted on a $70 \mu \mathrm{M}$ cell strainer using a $1 \mathrm{~mL}$ syringe plunger and cells were washed with IMDM followed by centrifugation at $320 \mathrm{~g}$ for $4 \mathrm{~min}$. LN cells were then resuspended in the appropriate buffer and stored at $4^{\circ} \mathrm{C}$. Before use samples were counted as described in section 2.2.6.6.

When stated, LN cells were digested to allow maximum release of cells. LN were pulled apart using 18 gauge needles and were incubated for $1 \mathrm{hr}$ at $37^{\circ} \mathrm{C}$ in $1 \mathrm{~mL}$ of IMDM containing 2.4 $\mathrm{mg} / \mathrm{mL}$ Collagenase II and $10 \mu \mathrm{g} / \mathrm{mL}$ DNase I. Cells were then forced repeatedly through a 21-gauge needle to obtain a single cell suspension, passed through $70 \mu \mathrm{M}$ gauze and collected into FACS tubes. Cells were then centrifuged at $320 \mathrm{~g}$ for $4 \mathrm{~min}$ and washed and resuspended in the appropriate buffer and stored at $4{ }^{\circ} \mathrm{C}$. Before use samples were counted as described in section 2.2.6.6. 


\subsubsection{Spleen}

Spleens were harvested from mice euthanised by either asphyxiated in $\mathrm{CO}_{2}$ or cervical dislocation. Spleens were placed into cIMDM and stored on ice until they could be processed.

Spleens were disrupted on a $70 \mu \mathrm{M}$ cell strainer using a $1 \mathrm{~mL}$ syringe plunger and cells were washed with IMDM and centrifuged at $320 \mathrm{~g}$ for $4 \mathrm{~min}$. Splenocytes were incubated in $3 \mathrm{~mL}$ ACT Buffer for 5 minutes at $37^{\circ} \mathrm{C}$ to lyse red blood cells followed by centrifugation at $320 \mathrm{~g}$ for $4 \mathrm{~min}$. Splenocytes were then resuspended in the appropriate buffer and stored at $4{ }^{\circ} \mathrm{C}$. Before use samples were counted as described in section 2.2.6.6.

When indicated spleens were digested to allow maximum release of splenocytes. $1 \mathrm{~mL}$ of IMDM containing $0.41 \mathrm{mg} / \mathrm{mL}$ liberase CI and $10 \mu \mathrm{g} / \mathrm{mL}$ DNase I was injected into the spleens using a $1 \mathrm{~mL}$ syringe and a 25 -gauge needle. Spleens were placed into either a 24 well plate or $15 \mathrm{~mL}$ Falcon tubes and incubated for $25 \mathrm{~min}$ at $37^{\circ} \mathrm{C}$. EDTA was then added to a final concentration of $10 \mathrm{mM}$ and spleens were incubated for a further $5 \mathrm{~min}$ at $37^{\circ} \mathrm{C}$. Spleens were then disrupted as above and treated with ACT buffer and resuspended in the appropriate buffer and stored at $4{ }^{\circ} \mathrm{C}$. Before use samples were counted as described in section 2.2.6.6.

\subsubsection{Thymus}

Thymi were harvested from mice euthanised by asphyxiation in $\mathrm{CO}_{2}$ and placed into cIMDM and stored on ice until samples could be processed.

Thymi were disrupted on a $70 \mu \mathrm{M}$ cell strainer using a $1 \mathrm{~mL}$ syringe plunger and cells were washed with IMDM and centrifuged at $320 \mathrm{~g}$ for $4 \mathrm{~min}$. Thymocytes were then resuspended in the appropriate buffer and stored at $4{ }^{\circ} \mathrm{C}$. Before use samples were counted as described in section 2.2.6.6. 


\subsubsection{Cell counting}

All cell samples were counted, either by Trypan blue dye (GIBCO) exclusion using a haemocytometer (Weber Scientific International Ltd) or with the Z2 Coulter Particle Count and Size Analyzer (Beckman Coulter Inc., Fullerton, CA, USA).

\subsubsection{Fluorescent labelling of cells and analysis by flow cytometry}

\subsubsection{Labelling of proteins and antibodies}

Purified antibodies or ova protein were labelled with Alexa Fluor 647 (Invitrogen), fluorescein isothiocyanate (FITC, Sigma) or NHS-Biotin (Biotin, Pierce, Rockford, IL, USA). The dyes were dissolved in DMSO to a concentration of $10 \mathrm{mg} / \mathrm{mL}$ and stored under oil at $-80^{\circ} \mathrm{C}$. Protein $(0.25-0.5 \mathrm{mg})$ was transferred to a Vivaspin 500 column (Vivascience, Stonehouse, UK) and concentrated by centrifugation at $4700 \mathrm{~g}$ for $10 \mathrm{~min}$. The protein was washed twice by adding $500 \mu \mathrm{L}$ fresh $0.1 \mathrm{M}$ sodium bicarbonate (Sigma, St Louis, MO, USA) diluted in $\mathrm{dH}_{2} \mathrm{O}$, followed by centrifugation at $4700 \mathrm{~g}$ for $10 \mathrm{~min}$ and discarding of the flow through. For labelling, the protein was transferred to a clean $1.7 \mathrm{~mL}$ microtube, and diluted to $2.5 \mathrm{mg} / \mathrm{mL}$ in $0.1 \mathrm{M}$ sodium bicarbonate buffer. The dye at $0.1 \mu \mathrm{g}$ per $1 \mu \mathrm{g}$ protein was added while mixing. After 8 min incubation at RT the protein was transferred back to the column and washed once with $0.1 \mathrm{M}$ Tris buffer ( $\mathrm{pH} 8-9$ ) and twice with PBS to remove any excess dye before transferring to a new tube. Labelled antibodies or ova protein were diluted to $0.5-1 \mathrm{mg} / \mathrm{mL}$ in PBS containing $0.1 \%$ sodium azide for storage at $4{ }^{\circ} \mathrm{C}$. Antibodies were then titrated to determine the optimal concentration for flow cytometric analysis.

\subsubsection{Detection of cell surface marker expression}

Single cell suspensions were prepared, washed once in FACS buffer and resuspended at 1-10 x $10^{7}$ cells $/ \mathrm{mL}$. The cell suspension was distributed to the wells of a round bottom 96 well plate in $100 \mu \mathrm{L}$ aliquots. The plate was centrifuged at $240 \mathrm{~g}$ for $2 \mathrm{~min}$ and the supernatants were tipped off by flicking the plate once. Pellets were resuspended by vortexing. Fc receptor blocking antibody (2.4G2) was added at $10 \mu \mathrm{g} / \mathrm{mL}$, and the plate was incubated at $4{ }^{\circ} \mathrm{C}$ for 10 
min. Fluorescently-conjugated antibodies against surface markers of interest were then added at appropriate dilutions in a volume of $50 \mu \mathrm{L}$ and incubated at $4{ }^{\circ} \mathrm{C}$ for a further $10 \mathrm{~min}$. Following the incubation $100 \mu \mathrm{L}$ of FACS buffer was added to each well and the plate centrifuging at $240 \mathrm{~g}$ for $2 \mathrm{~min}$ to remove any excess antibody. If required, second step reagents were added and incubated for a further $10 \mathrm{~min}$ at $4{ }^{\circ} \mathrm{C}$. Cells were washed and resuspended in $200 \mu \mathrm{L}$ of FACS buffer, containing PI at $0.25 \mu \mathrm{g} / \mathrm{mL}$ or $0.1 \mu \mathrm{g} / \mathrm{mL}$ DAPI, for analysis by flow cytometry.

\subsubsection{Detection of antigen specific TCR using MHC I pentamers}

Samples were prepared as described in section 2.2.6 and cells placed in the wells of a round bottom 96 well plate. As described in section 2.2.7.2 the Fc receptors were blocked using the $2.4 \mathrm{G} 2$ antibody followed by the addition of $100 \mu \mathrm{L}$ of FACS buffer to each well and centrifuged at $240 \mathrm{~g}$ for $2 \mathrm{~min}$. After centrifugation the supernatants were tipped off by flicking the plate once.

For single labelling with the unlabelled $\mathrm{D}^{\mathrm{b}}-\mathrm{NP}_{366-374}$ pentamer an optimal concentration was added directly to the cells and incubated for $10 \mathrm{~min}$ at RT. Cells were then washed and the supernatants tipped off. $8 \mu \mathrm{L}$ of the R-PE Fluorotag was added to the cells along with any other antibodies of interest at the appropriate concentration and incubated at $4{ }^{\circ} \mathrm{C}$ for $20 \mathrm{~min}$. Cells were then washed and resuspended in $200 \mu \mathrm{L}$ of FACS buffer, containing PI at 0.25 $\mu \mathrm{g} / \mathrm{mL}$, for analysis by flow cytometry.

If samples were to be labelled with both unlabelled $\mathrm{D}^{\mathrm{b}}-\mathrm{NP}_{366-374}$ and biotin $\mathrm{D}^{\mathrm{b}}-\mathrm{PA}_{224-233}$ pentamers, cells were labelled as above with the $\mathrm{D}^{\mathrm{b}}-\mathrm{NP}_{366-374}$. Then the biotin labelled $\mathrm{D}^{\mathrm{b}}$ $\mathrm{PA}_{224-233}$ pentamer was then added at $2.5 \mu \mathrm{L} / \mathrm{sample}$ for $10 \mathrm{~min}$ at RT. Cells were washed and supernatant flicked off. SA-APCy was added along with an anti-CD8 antibody at the appropriate concentration and incubated at $4^{\circ} \mathrm{C}$ for $20 \mathrm{~min}$. Cells were then washed and resuspended in $200 \mu \mathrm{L}$ of FACS buffer, containing PI at $0.25 \mu \mathrm{g} / \mathrm{mL}$, for analysis by flow cytometry. 
For single labelling with the PE conjugated $\mathrm{K}^{\mathrm{b}}$-ova $257-264$ pentamer, an optimal concentration was added directly to the cells and incubated for $10 \mathrm{~min}$ at RT. Cells were then washed and the supernatants tipped off. Other cell surface antibodies were then added at their optimal concentrations and incubated at $4{ }^{\circ} \mathrm{C}$ for $20 \mathrm{~min}$. Cells were then washed and resuspended in $200 \mu \mathrm{L}$ of FACS buffer, containing PI at $0.25 \mu \mathrm{g} / \mathrm{mL}$, for analysis by flow cytometry.

\subsubsection{Loading of CD1d tetramer with $\alpha$-GalCer and detection of NKT cells with the CD1d tetramer}

The PE labelled mouse CD1d tetramer was loaded with $\alpha$-GalCer (Alexis) by mixing with 12 molar excess of $\alpha$-GalCer that had been sonicated for $2 \mathrm{hr}$ at $37{ }^{\circ} \mathrm{C}$ and incubated overnight at RT. Before labelling of other surface markers (see section 2.2.7.2), cells were incubated for 30 min with the $\alpha$-GalCer-loaded mouse CD1d tetramer (ProImmune) at $4{ }^{\circ} \mathrm{C}$. Cells were then washed and resuspended in $400 \mu \mathrm{L}$ of FACS buffer, containing DAPI at $0.1 \mu \mathrm{g} / \mathrm{mL}$, for analysis by flow cytometry.

\subsubsection{Detection of intracellular IFN- $\gamma$ after in vitro restimulation}

\subsection{In vitro restimulation}

Prior to intracellular labelling of IFN- $\gamma$, cells were resuspended in cIMDM at 1-2 x $10^{6}$ cells $/ \mathrm{mL}$ and placed into 6 or 24 well plates, with $1 \mu \mathrm{M} \mathrm{NP}$ 366-374 or $\mathrm{PA}_{224-233}$ peptide. GolgiStop (Pharmingen, BD Biosciences) was added at the beginning of the restimulation at 1 $\mu \mathrm{g} / \mathrm{mL}$, to prevent export of proteins from the Golgi bodies. Cells were incubated at $37^{\circ} \mathrm{C}$ for 4-6 hr. Cells were then harvested and washed in IMDM.

\subsection{Detection of intracellular IFN- $\gamma$}

After peptide stimulation, cells were labelled with antibodies for cell surface markers, before fixing for intracellular labelling. After surface labelling as described in section 2.2.7.2, cells were fixed and permeabilised using the BD Cytofix/Cytoperm Kit (Pharmingen, BD 
Biosciences). Briefly, cells were washed in FACS buffer and fixed by incubating for $20 \mathrm{~min}$ with $200 \mu \mathrm{L}$ of BD Cytofix/Cytoperm solution at RT. Cells were then washed twice with $1 \mathrm{X}$ BD Perm/Wash buffer. The antibody for IFN- $\gamma$, or its respective isotype control antibody, was added in $50 \mu \mathrm{L}$ Cytofix/Cytoperm solution and cells were incubated for $30 \mathrm{~min}$ at $4{ }^{\circ} \mathrm{C}$. Cells were washed twice more in cold BD Perm/Wash Buffer, resuspended in $200 \mu$ L FACS buffer and kept at $4{ }^{\circ} \mathrm{C}$ before analysis by flow cytometry.

\subsubsection{Acquisition and analysis}

After surface labelling live cells were resuspended in FACS buffer containing PI or DAPI, for acquisition. Live cells were identified based on Forward Scatter (FSC) and Side Scatter (SSC) properties and the exclusion of $\mathrm{PI}^{+}$or $\mathrm{DAPI}^{+}$events.

Fixed cells were resuspended in $200 \mu \mathrm{L}$ of FACS buffer for acquisition. Intact, fixed cells were identified based on FSC versus SSC properties. Unlabelled samples and single labelled controls for each fluorochrome were included in all cases for calibration of voltages and compensation. Isotype control antibodies were used to control for background fluorescence caused by non-specific antibody binding as indicated.

Data was acquired using either a BD FACSCalibur or BD LSRII SORP (both BecktonDickinson, Mountain View, CA, USA) and analysed using FlowJo software (Tree Star, San Carlos, CA, USA).

\subsubsection{Cell purification by Magnetic Cell Separation (MACS)}

Single cell suspensions of OT-IxB6.congenic LN, filtered through nylon gauze to remove cell clumps, were enriched for $\mathrm{CD} 8^{+} \mathrm{T}$ cells using the AutoMACS system (Miltenyi Biotec, Bergisch Gladbach, Germany). Ten million cells were resuspended in $90 \mu \mathrm{L}$ Würzburger buffer with $10 \mu \mathrm{L}$ MACS CD8 $\alpha$ Microbeads (Miltenyi Biotec) per $100 \mu \mathrm{L}$. Cells and beads 
were mixed well and incubated for $15 \mathrm{~min}$ at $4{ }^{\circ} \mathrm{C}$. Cells were washed by adding $1 \mathrm{~mL}$ of Würzbuger buffer followed by centrifugation at $320 \mathrm{~g}$ for $4 \mathrm{~min}$ and scaled up as appropriate. Cells were then resuspended in $500 \mu \mathrm{L}$ Würzbuger buffer per $1 \times 10^{8}$ cells and filtered through a $40 \mu \mathrm{m}$ cell strainer before positive selection on the AutoMACS. Positively selected cells were resuspended in PBS for i.v. injection.

\subsubsection{Neutralisation of TNF- $\alpha$}

Mice were treated i.p. with $10 \mathrm{mg} / \mathrm{kg}$ of anti-TNF- $\alpha$ (clone TN3-19.12, BioLegend, San Diego, CA, USA). Mice were treated i.v. with $200 \mathrm{ng} \alpha$-GalCer $20 \mathrm{hr}$ later. Then $48 \mathrm{hr}$ after the $\alpha$-GalCer treatment spleens were harvested, digested and processed as described in section 2.2.6.4. To confirm that the anti-TNF- $\alpha$ treatment was effective, blood was collected at $3 \mathrm{hr}$ after $\alpha$-GalCer treatment, and the serum level of TNF- $\alpha$ measured using the Bio-Plex ${ }^{\mathrm{TM}}$ system (Bio-Rad Laboratories, Hercules, CA, USA) according to manufacturer's instructions (see section 2.2.10).

\subsubsection{Detection of serum cytokines}

Blood was taken from mice by slicing the lateral tail vein and 5-8 drops collected in $1.7 \mathrm{~mL}$ microtubes. The blood was incubated at RT for 4-6 hr to allow blood clotting. After centrifugation at $14000 \mathrm{~g}$ for 5 min serum was transferred to a new microtube and stored at $20{ }^{\circ} \mathrm{C}$ until analysis. Serum levels of IL-4, IFN- $\gamma$, IL-12p70 and TNF- $\alpha$ were determined using a bead multiplex immunoassay (Bio-Plex ${ }^{\mathrm{TM}}$ systems, Bio-Rad Laboratories) according to manufacturer's instructions. Briefly, the desired capture beads, $0.65 \mu \mathrm{L}$ per cytokine were combined in a microtube in a total volume of $50 \mu \mathrm{L} \mathrm{Bio-Plex}^{\mathrm{TM}}$ assay buffer and transfer to 96 well filter plates (L-Plate, Abacus ALS, Auckland, New Zealand). The plate was washed twice with Bio-Plex ${ }^{\mathrm{TM}}$ assay buffer by adding $100 \mu \mathrm{L}$ buffer per well and removing by vacuum (Bio-Rad). Serum samples were diluted either 1:2 or 1:4 in Bio-Plex ${ }^{\mathrm{TM}}$ assay buffer to a final volume of $50 \mu \mathrm{L}$ and added to the plate. The plate was then incubated with agitation for 
30 min at RT and then washed twice as described above. Detection antibodies diluted appropriately in Bio-Plex ${ }^{\mathrm{TM}}$ assay buffer and $25 \mu \mathrm{L}$ was added to the plate followed by washing and incubation with $50 \mu \mathrm{L}$ of SA-PE for $10 \mathrm{~min}$ at RT. Samples were then washed and resuspended in $125 \mu \mathrm{L}$ Bio-Plex ${ }^{\mathrm{TM}}$ assay buffer before analysis using the Bio-Plex ${ }^{\mathrm{TM}}$ system. Cytokine concentrations were determined against commercial standards (Bio-Rad) and calculated using the provided software (Bio-Rad, Bio-Plex ${ }^{\mathrm{TM}}$ systems).

\subsubsection{Detection of ova-specific antibodies by ELISA}

\subsubsection{Generation of positive control serum}

A positive control was used to confirm that the ELISA was working correctly. Mice were primed i.p. with two doses of $2 \mu \mathrm{g}$ ova protein (Sigma) in $100 \mu \mathrm{L}$ alum $\left(2 \% \mathrm{Al}(\mathrm{OH})_{3}\right.$ equivalent to $1.3 \% \mathrm{Al}_{2} \mathrm{O}_{3}$, Serva Electrophoresis $\mathrm{GmbH}$, Heidelberg, Germany). Mice received the first dose of alum and ova protein then 14 days later boosted again. Then 10 days after the second dose of alum and ova protein cardiac puncture was performed and blood collected in $1.7 \mathrm{~mL}$ microtubes. Serum was then collected as described in section 2.2.10.

\subsubsection{Ova-specific IgG1 ELISA of murine serum}

Initially, Maxi sort immuno binding 96 Microwell plates were coated overnight at $4{ }^{\circ} \mathrm{C}$ with $100 \mu \mathrm{L}$ of ova protein diluted in ELISA coating buffer to a final concentration of $1 \mu \mathrm{g} / \mathrm{well}$. After overnight incubation plates were inverted to remove excess solution. Wells were then washed 4 times with ELISA Wash Buffer and incubated with $150 \mu \mathrm{L}$ of ELISA Blocking Buffer for $1 \mathrm{hr}$ at RT to block non-specific protein binding.

Serum samples were prepared while plates were blocking. Serum samples were thawed on ice and samples diluted in PBS. Samples were initially diluted 1:10 then 1:2 serial dilutions were carried out to a final dilution of 1:640. After blocking, plates were inverted to remove any excess solution and $50 \mu \mathrm{L}$ of the appropriate samples were added to each well. Plates were 
sealed, placed in the dark for $1 \mathrm{hr}$ at RT. Plates were washed 6 times with ELISA Wash Buffer to remove any unbound serum proteins. For detection of ova-specific IgG1, $50 \mu \mathrm{L}$ of biotin rat anti-mouse IgG1 antibody diluted 1:1000 in ELISA Buffer was added to each well and plates were incubated for $2 \mathrm{hr}$ at RT in the dark. To remove excess unbound antibody plates were washed as described above. Then the second step reagent, streptavidin-horseradish peroxidase (HRP) was diluted 1:1000 in ELISA Buffer and $100 \mu \mathrm{L}$ added to each well followed by $1 \mathrm{hr}$ incubation in the dark at RT. After the $1 \mathrm{hr}$ incubation plates were washed as described above to remove excess unbound streptavidin-HRP. Lastly, $100 \mu \mathrm{L}$ of ELISA Substrate Buffer was added to each well for 5-10 min at RT until a gradient of colour was observed across the positive control sample, at which point $50 \mu \mathrm{L}$ of ELISA Stopping Solution was added to all wells. After stopping the reactions, the absorbance of each well was read at $414 \mathrm{~nm}$ using a Versa $_{\max }$ Microplate reader (Molecular Devices, Sunny Vale, CA, USA). Antibody titer was calculated using SOFTmax ${ }^{\circledR}$ PRO (Molecular Devices).

\subsubsection{In vivo VITAL assay for cytotoxicity}

The VITAL assay provides a method for assessing antigen specific cytotoxicity by the analysis of elimination of fluorescently-labelled, antigen-loaded target cells compared to a control population of antigen-negative cells (360).

\subsubsection{Peptide loading of splenic targets}

Single cell suspensions were prepared from the spleens of C57BL/6 mice and separated into 4 equal aliquots. One group of cells was incubated with $\alpha$-GalCer at a final concentration of 6 $\mu \mathrm{M}$, the second group received $0.6 \mu \mathrm{M} \alpha$-GalCer, while the third group received $60 \mathrm{nM} \alpha-$ GalCer. The remaining group was left untreated. Cells were incubated at $37{ }^{\circ} \mathrm{C}$ for $2 \mathrm{hr}$, mixing every half hour by flicking or inverting tubes. Cells were then centrifuged at $320 \mathrm{~g}$ for 4 min. 


\subsubsection{Labelling of cells with VITAL dyes}

Untreated cells were labelled with the dye CTO. Cells were counted and resuspended in prewarmed cIMDM at $5 \times 10^{6}$ cells $/ \mathrm{mL}$. The CTO dye was added to the cells at a final concentration of $10 \mu \mathrm{M}$ and incubated at $37^{\circ} \mathrm{C}$ for $15 \mathrm{~min}$ followed by centrifugation at $320 \mathrm{~g}$. To remove excess dye cells were resuspended in warmed cIMDM and incubated for $20 \mathrm{~min}$ at $37^{\circ} \mathrm{C}$. The tube was then centrifuged at $320 \mathrm{~g}$ and resuspended in IMDM.

Cells treated with $\alpha$-GalCer were labelled with the dye CFSE. Cells were counted and resuspended in cold PBS at $5 \times 10^{6}$ cells/mL. CFSE was added to cells treated with $6 \mu \mathrm{M} \alpha$ GalCer at a final concentration of $4 \mu \mathrm{M}$, and cells treated with $0.6 \mu \mathrm{M}$ and $60 \mathrm{nM} \alpha$-GalCer were labelled with $0.8 \mu \mathrm{M}$ and $0.16 \mu \mathrm{M}$ CFSE respectively. Tubes were briefly vortexed to evenly distribute dye and incubated for $10 \mathrm{~min}$ at $37^{\circ} \mathrm{C}$. Five volumes of cold PBS were added to each tube to stop the labelling reaction and tubes were centrifuged at $320 \mathrm{~g}$ and resuspended in IMDM.

All 4 tubes of cells were then washed 3 times in IMDM prior to i.v. injection. Each set of cells was counted and resuspended in IMDM at $6 \times 10^{7}$ cells $/ \mathrm{mL}$. The 4 groups of cells were then pooled together at equal ratios to give a final concentration of $6 \times 10^{7}$ cells $/ \mathrm{mL}$.

C57BL/6, PKO and CD1 KO mice each received $200 \mu \mathrm{L}$ i.v. of the target cell mixture.

\subsubsection{Analysis of NKT cell killing}

Killing was measured at $24 \mathrm{hr}$ after administration of target cells. At this time point the MesLN were collected and made into a single cell suspension by straining through $70 \mu \mathrm{M}$ gauze. Cells were then resuspended in $500 \mu \mathrm{L}$ FACS buffer for analysis by flow cytometry. The amount of specific killing of $\alpha$-GalCer-loaded target cells at each $\alpha$-GalCer concentration was determined by calculating the ratio of surviving $\alpha$-GalCer-loaded target cells $\left(\mathrm{CFSE}^{+}\right)$ versus untreated target cells $\left(\mathrm{CTO}^{+}\right)$and adjusted by comparing to $\mathrm{CD} 1 \mathrm{KO}$ mice. The $\%$ specific killing was calculated as follows: 
$\%$ specific killing $=\underline{100-100 \times \mathrm{CFSE}^{+} \text {cells (sample) } / \mathrm{ZCTO}^{+} \text {cells (sample) }}$

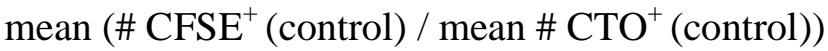

\subsubsection{Challenge with B16.ova tumour cells}

Mice were injected s.c. in the left flank with 1 x $10^{5}$ B16.ova tumour cells. Tumours in untreated control mice were palpable from $~ 8-10$ days after tumour challenge. Once tumours were palpable they were checked 3 times a week. Bisecting diameters were measured using Mitutoyo callipers, with $1.5 \mathrm{~mm}$ being subtracted to account for the skin fold thickness. Mice were euthanised when tumour size reached $150-200 \mathrm{~mm}^{2}$.

\subsubsection{Statistical calculations}

All statistical analysis was carried out assuming that the data was sampled from populations that approximately followed a Gaussian bell-shaped distribution. Tests for normality were conducted on the number of $\mathrm{CD} 8^{+}$and $\mathrm{CD} 8^{-} \mathrm{DC}$ subsets before and after $\alpha$-GalCer treatment and the populations were found to be distributed normally. Parametric tests were chosen as when analysing biological data for which the sample size is small $(<12)$ they are more powerful than non-parametric tests which have little power to detect differences no matter how the values differ (361). The statistical significance of differences between 3 or more groups of data was calculated using either a one-way analysis of variance (ANOVA) followed by a Tukey post test to compare all groups of data or a two-way ANOVA followed by a Bonferroni post test to compare all groups of data. Statistical significance of differences between 2 groups of data was calculated using a Student's $t$ test using a $99 \%$ confidence interval, as indicated. Statistical calculations were performed using the Graphpad Prism ${ }^{\circledR}$ Version 4 statistical package (Graphpad Software Inc., San Diego, CA, USA), and the companion statistics guide (361). 


\section{Chapter 3:}

Characterisation of immune responses to influenza A virus in PKO mice 


\subsection{Introduction}

As discussed in detail in Chapter 1, DC are critical for the initiation of $\mathrm{CD}^{+}$and $\mathrm{CD} 8^{+} \mathrm{T}$ cell responses. It has been demonstrated that CTL-mediated DC elimination has a detrimental effect on T cell proliferation and tumour immunity (169). Improved DC survival through manipulation of the expression of anti-apoptotic molecules has been shown to enhance $\mathrm{CD}^{+}$and $\mathrm{CD}^{+} \mathrm{T}$ cell responses $(144,158,160,161)$ and improve tumour protection (160). However, not all situations of extended DC survival result in a more desirable outcome as reports have linked increased DC survival to chronic lymphocyte activation and associated immunopathology $(158,163,164,362,363)$. Therefore it appears that carefully regulated DC survival is essential for the generation of effective $\mathrm{T}$ cell mediated immunity.

Through the use of different experimental systems it has been proposed that DC presenting antigen are eliminated by responding CTL, thereby limiting the duration of antigen presentation and hence the magnitude of the immune response $(153,155,336)$. Direct evidence supporting this has come from investigations demonstrating that DC loaded with antigen then injected into immune C57BL/6 mice are rapidly eliminated by TCR transgenic CD ${ }^{+} \mathrm{T}$ cells $(155,169,345,364)$. Furthermore, when antigen loaded BMDC were injected into immune PKO mice, whose CTL cannot kill target cells using the perforin/granzyme pathway, the BMDC could be recovered 24-96 hr later (155), indicating that CTL regulate DC survival in a perforin-dependent manner. However, it is important that these results are confirmed using a more physiologically relevant model.

The murine influenza A virus model was chosen as a physiologically relevant model to assess how CTL regulate DC survival for several reasons. Firstly, infected mice represent a highly reproducible model for analysing $\mathrm{CD}^{+} \mathrm{T}$ cell mediated immunity (365). Secondly, the influenza A virus infection has been well characterised and a number of reagents are available to enable assessment of the influenza-specific immune response. Thirdly, this model allows the assessment of perforin in CTL-mediated killing as PKO mice are able to clear influenza A virus, albeit slightly more slowly than infected C57BL/6 mice (366). Finally, both primary and secondary immune responses can be easily assessed. This is important because both CTL and memory $\mathrm{CD}^{+} \mathrm{T}$ cells have been shown to kill DC, however, their relative contribution to DC elimination has not been shown in a 
physiologically relevant setting $(169,344,345)$. Therefore these features of the murine model of influenza infection make it an excellent physiologically relevant model to assess the role of CTL in DC killing.

To investigate a secondary influenza-specific $\mathrm{CD}^{+} \mathrm{T}$ cell response two heterologous viruses were used, PR8 (H1N1) and HKx31 (H3N2). These viruses share the internal proteins; so prior infection with the PR8 virus generates populations of memory $\mathrm{CD}^{+} \mathrm{T}$ cells that are reactivated when mice are challenged with the HKx31 virus $(354,355,357)$. In contrast, the external proteins differ between the two viruses, enabling a secondary infection to be established in mice that carry high titres of neutralizing antibodies. In this thesis two different routes of administration were used for the influenza viruses. Intranasal (i.n.) administration results in a productive viral infection in respiratory epithelial cells (367) correlating with an observed weight loss, whereas intraperitoneal (i.p.) administration leads to a robust influenza-specific $\mathrm{CD}^{+} \mathrm{T}$ cell response without the establishment of a productive viral infection and the consequent side effects. This route of administration is used preferentially for virulent isolates such as the PR8 virus.

The influenza-specific immune response can be assessed by a number of parameters. Initially the weight loss of infected mice can be evaluated to determine the progression of the viral infection, while measuring the viral load in lung can be to determine the kinetics of viral clearance. Additionally, the kinetics of the influenza-specific $\mathrm{CD} 8^{+} \mathrm{T}$ cell response can be easily assessed. The percentage of antigen-specific $\mathrm{CD}^{+} \mathrm{T}$ cells was measured by two methods, firstly by using fluorescently-labelled MHC I pentamers, secondly by measuring the specific synthesis of IFN- $\gamma$. Two dominant $\mathrm{CD}^{+}$epitopes have been characterised in the murine influenza A virus model. The $\mathrm{CD}^{+}$epitope from the viral nucleoprotein (NP) $\mathrm{NP}_{366-374}$, is co-dominant with an epitope from the acid polymerase protein, $\mathrm{PA}_{224-233}$, during a primary influenza immune response $(354,356)$, while the $\mathrm{D}^{\mathrm{b}}$ $\mathrm{NP}_{366-374}$ epitope is the dominant epitope during a secondary response $(354,355,357)$. Furthermore, the phenotype of antigen-specific $\mathrm{CD} 8^{+} \mathrm{T}$ cells can be evaluated by flow cytometry. The cell surface marker, CD62L, is primarily expressed on naive T cells and a subset of resting memory cells and its absence can be used as an indicator of an effector phenotype (197). Additionally, the marker Killer Cell Lectin-like Receptor G 1 (KLRG1) has been associated with terminally differentiated memory $\mathrm{CD} 8^{+} \mathrm{T}$ cells and $\mathrm{T}$ cell exhaustion (368-371). Thus evaluating the expression of KLRG1 and CD62L on 
influenza-specific $\mathrm{CD}^{+} \mathrm{T}$ cells provides further insight into the phenotype and differentiation state of these cells.

\subsection{Aims}

The experiments described in this chapter were designed to characterise and compare antigen-specific immune responses in C57BL/6 and PKO mice. The murine influenza A virus was chosen as a physiologically relevant immune response to evaluate this in. It was hypothesised that compared to C57BL/6 mice, PKO mice would have an enhanced influenza specific $\mathrm{CD}^{+} \mathrm{T}$ cell response.

The specific aims were:

- To profile the progression of the influenza virus infection in C57BL/6 mice by measuring the severity of disease and the rate of viral clearance, and comparing it to that of PKO mice.

- To determine whether PKO mice have an enhanced antigen-specific CD8 ${ }^{+} \mathrm{T}$ cell response. The influenza-specific $\mathrm{CD}^{+} \mathrm{T}$ cell response during both a primary and secondary infection in both C57BL/6 and PKO mice was investigated to address this aim.

- To gain information about the phenotype and differentiation status of influenza specific T cells by comparing the expression of CD62L and KLRG1 on influenzaspecific $\mathrm{CD}^{+} \mathrm{T}$ cells in $\mathrm{C} 57 \mathrm{BL} / 6$ and $\mathrm{PKO}$ mice at specific time points during both a primary and secondary influenza infection. 


\subsection{Results}

\subsubsection{Influenza A virus establishes infections of comparable severity in C57BL/6 and PKO mice}

It has been previously published that PKO mice can clear the influenza A virus with almost similar kinetics to the C57BL/6 mice $(366,372)$; however, it was important to confirm this in our particular model setting. An initial primary indicator of influenza A viral infection is a decrease in body weight. To determine whether C57BL/6 and PKO mice might respond differently to a primary infection in the lung, the change in weight during the course of the infection was evaluated. Mice were weighed before the influenza infection followed by multiple weighings throughout the course of infection. The percentage weight loss was then determined. Maximal weight loss was observed seven days after the initial infection, where mice typically had lost $15-18 \%$ of their initial body weight (Figure 3.1 A). This weight loss was observed in both C57BL/6 and PKO mice. However, PKO mice were slower to re-gain their original weight compared to C57BL/6 mice. This delay in recovery was also observed in a repeat experiment (Figure 3.1 C), although the difference did not reach statistical significance. Throughout the later stages of the infection the C57BL/6 and PKO mice re-gained their weight at a similar rate (Figure 3.1 A). No weight loss was only observed when the influenza virus, PR8 was administered i.p. indicating that weight loss was a consequence of a productive infection (Figure $3.1 \mathrm{~B}$ ). This was consistent with previously published data that demonstrated the influenza virus was only able to establish a productive viral infection in the lung epithelia (367). Overall, these data indicate that the $\mathrm{C} 57 \mathrm{BL} / 6$ and $\mathrm{PKO}$ mice have an almost similar recovery rate after a primary influenza A virus infection.

Weight loss throughout a secondary influenza infection was also evaluated. Previously published data has demonstrated that PKO mice can clear a secondary viral challenge at a similar rate to C57BL/6 mice (372) but it was necessary to confirm this result in the current setting. To establish a secondary influenza infection mice were first primed with the PR8 virus i.p. and then 1 month later mice were challenged i.n. with the HKx31 virus. During the secondary influenza infection both mouse strains had a comparable maximal 
weight loss (10-12\%), which was observed five days after infection (Figure 3.1 C).

Furthermore, as they recovered, the C57BL/6 and PKO mice gained weight at a similar rate during the secondary influenza infection. Overall weight loss was comparable in both C57BL/6 and PKO mice during both a primary and secondary influenza viral infection. 


\section{Figure 3.1 Weight loss after primary and secondary influenza infection.}

C57BL/6 and PKO mice were infected as indicated with $\mathrm{HKx} 31$ and/or PR8 influenza virus and percentage weight loss relative to day 0 (pre-infection) was recorded at specific times after infection. (A) Mice were infected i.n. with HKx31 virus or mock-infected with PBS. Each data point represents mean + SD of 6 mice per group (B) Mice were infected i.p. with PR8 virus or mock-infected with PBS. Each data point represents mean + SD of 3-8 mice per group (C) Mice that had been infected i.p. with the PR8 virus 30 days earlier were re-infected i.n. with the HKx31 virus (Secondary) or previously uninfected mice were infected i.n. with the HKx31 virus only (Primary). As a negative control mice were mock-infected with PBS. Each data point represents mean + SD of 3-5 mice per group. *: indicates the statistical difference in C57BL/6 and PKO HKx31 infected mice, $p<0.05$ by a two-way ANOVA with a Bonferroni post test. The experiments were repeated two or more times with similar results. 
A. Weight loss in C57BL/6 and PKO mice

during a primary infection with $\mathrm{HK} \times 31$ i.n.

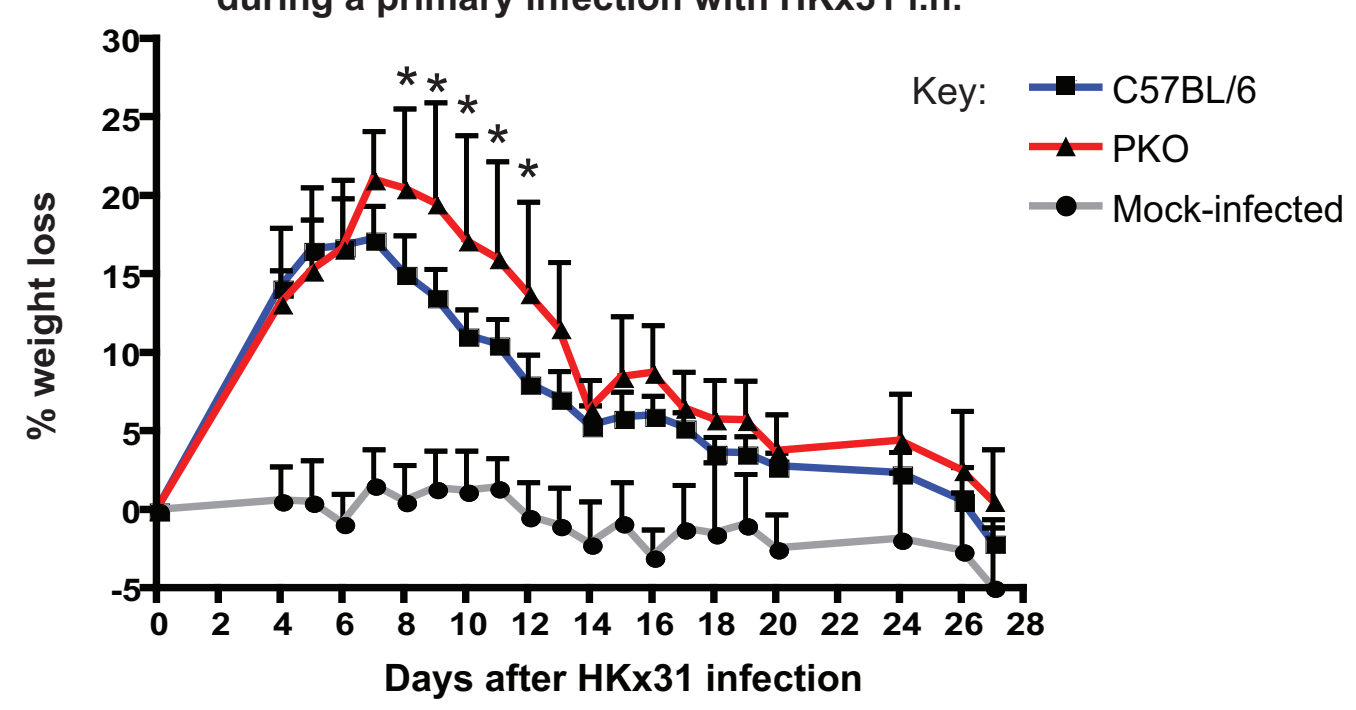

B. Weight loss in C57BL/6 and PKO mice during a primary infection with PR8 i.p.

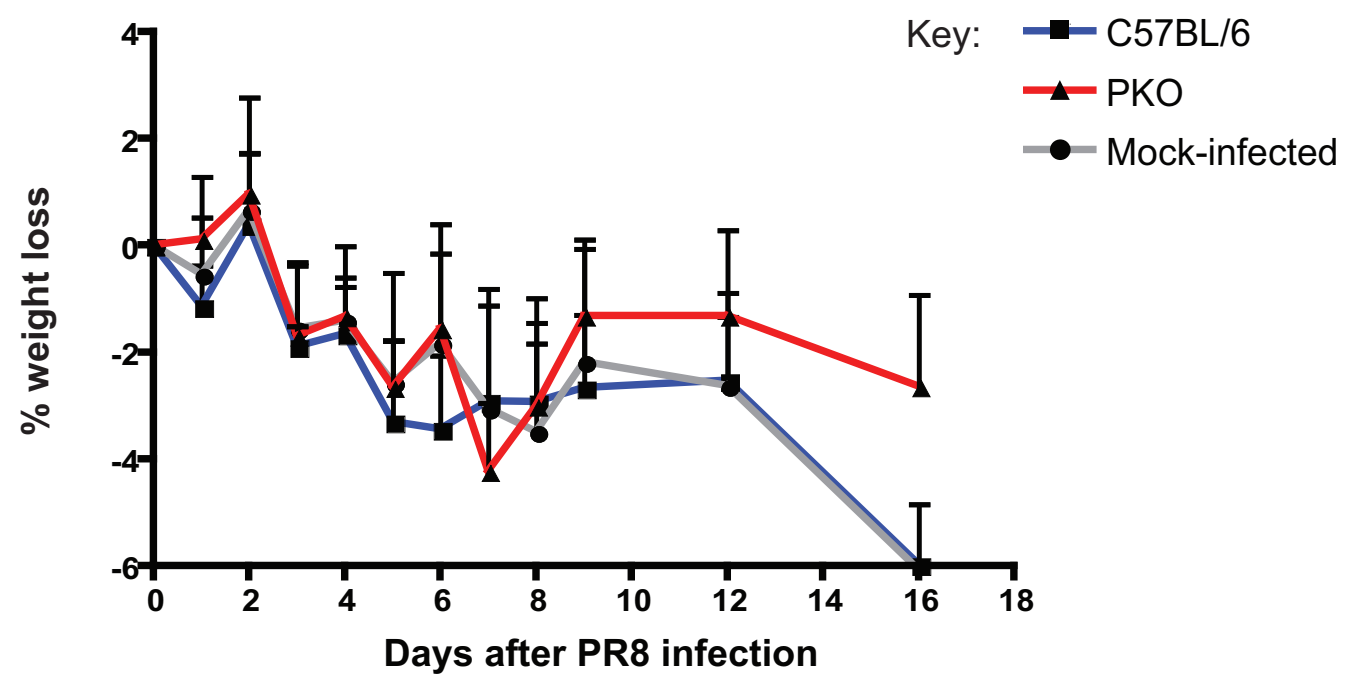

C. Weight loss in C57BL/6 and PKO mice during a secondary infection with HKx31 i.n.

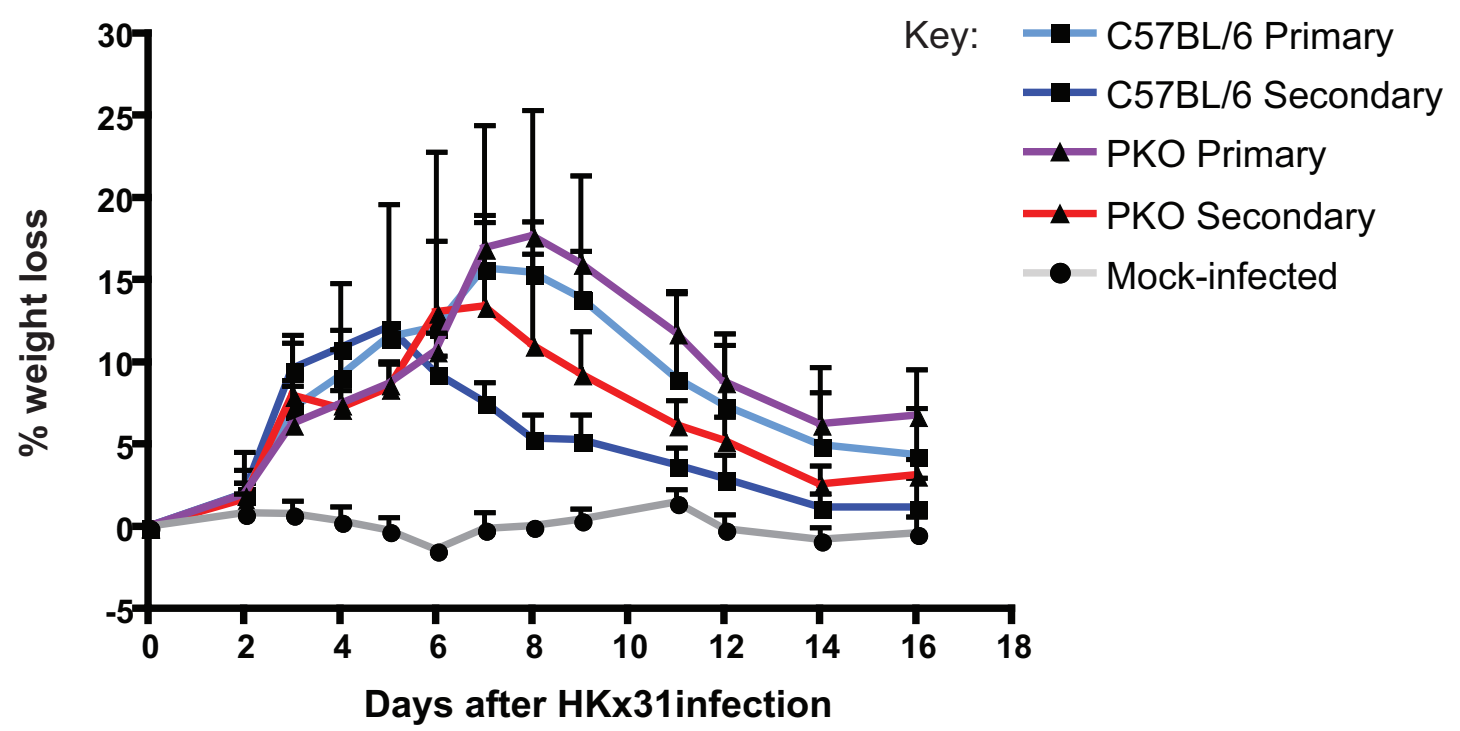




\subsubsection{Measurement of viral load in the lung tissue after a primary influenza infection}

In addition to measuring weight loss, the viral load present in the lung can also be assessed to determine the rate of viral clearance. Topham et al reported that PKO mice are able to clear influenza virus at a similar rate to C57BL/6 mice (366). To confirm this, semiquantitative real-time PCR was performed to investigate the level of viral RNA present in the lung tissue at various days after infection. Lung tissue was homogenised and RNA extracted using TRIzol and cDNA was prepared. Primers specific for the matrix protein (358), which is conserved across many influenza A virus strains, were used to determine the amount of viral RNA in the lung tissue. Five days after inoculation of the HKx31 virus i.n. both the C57BL/6 and PKO mice had a 100-fold higher lung viral RNA titer relative to the C57BL/6 mice that had been inoculated for eight days (Figure 3.2). This sample was chosen as the relative control because a sample that contained viral RNA was required. Eight days after HKx31 viral infection the PKO mice had a higher level of viral RNA present in the lung tissue compared to the C57BL/6 mice (Figure 3.2), although this difference did not reach statistical significance. Eleven and 14 days after virus infection both the PKO and C57BL/6 mice had barely detectable levels of virus in the lung tissue (Figure 3.2). These semi-quantitative RT-PCR results indicated that PKO mice are able to clear the $\mathrm{HKx} 31$ virus at an almost similar rate to $\mathrm{C} 57 \mathrm{BL} / 6$ mice. 


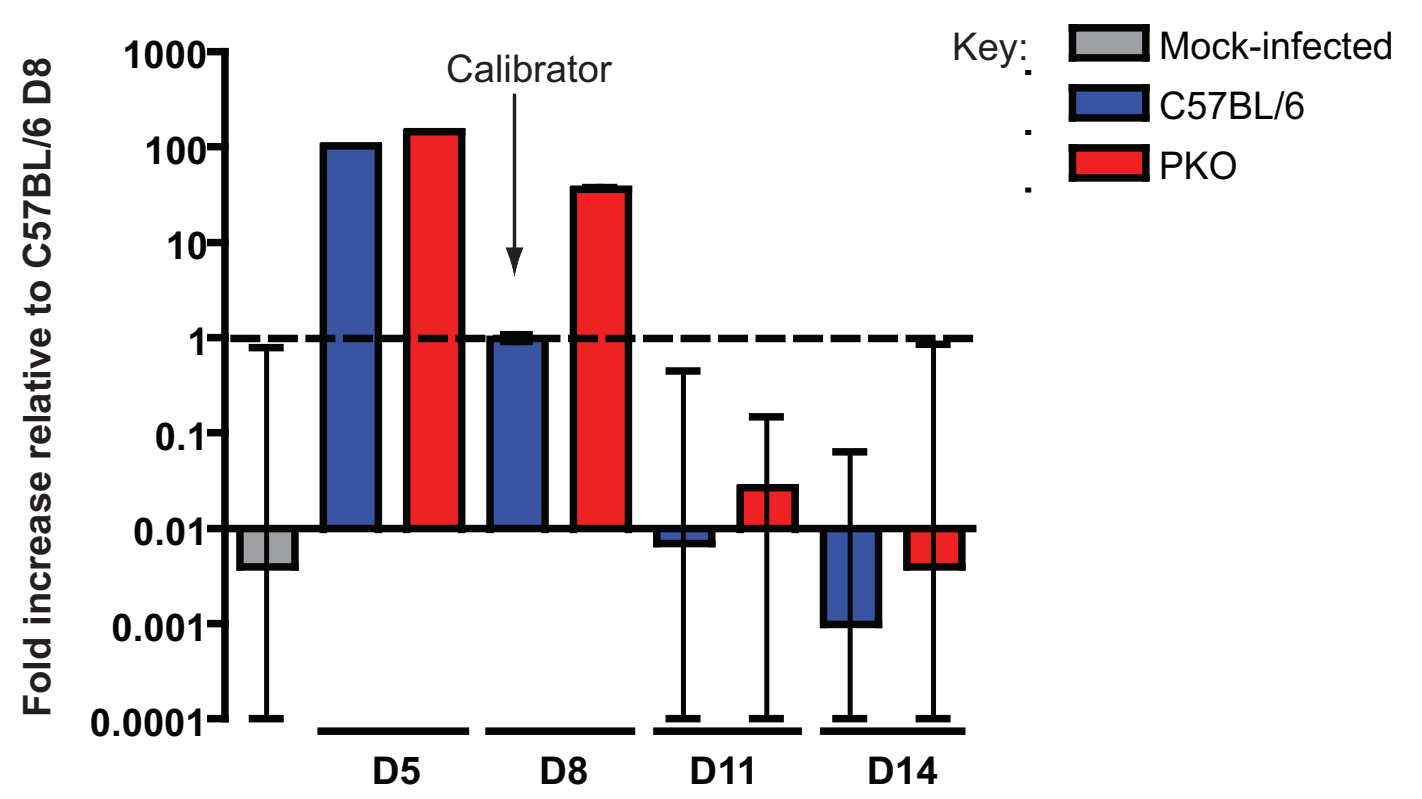

Figure 3.2 Clearance of the HKx31 virus from the lung tissue is slightly delayed in PKO mice compared to C57BL/6 mice.

Lung tissue was collected from C57BL/6 and PKO mice at specific time points after i.n. infection with the HKx31 virus. Lung tissue was homogenized and RNA extracted using TRIzol and cDNA was prepared for quantitative real-time PCR. Primers specific for the matrix protein were used to determine the amount of influenza A virus present in the lung tissue. Each sample was normalised to $18 \mathrm{~S}$ RNA. Data are shown as a fold increase relative to an arbitrarily chosen sample (C57BL/6 D8). Bars show mean \pm SD of 3 mice per group. No statistical difference between C57BL/6 and PKO mice was found by a one-way ANOVA. 


\subsubsection{Evaluation of the influenza-specific $\mathrm{T}$ cell response using MHC I pentamers in C57BL/6 and PKO mice during a primary infection}

The previous results illustrated that PKO mice could clear the virus at a comparatively similar rate to the $\mathrm{C} 57 \mathrm{BL} / 6$ mice. Next the antigen-specific $\mathrm{CD}^{+} \mathrm{T}$ cell response was investigated to determine whether PKO mice had an increased immune response compared to $\mathrm{C} 57 \mathrm{BL} / 6$ mice, indicating whether survival of antigen presenting DC is regulated in a perforin-dependent manner. Initially, the frequency of $\mathrm{CD}^{+} \mathrm{D}^{\mathrm{b}}-\mathrm{NP}_{366-374}{ }^{+} \mathrm{T}$ cells was examined in multiple tissues 10 days after HKx31 i.n. infection using a MHC I pentamer. To control for specificity, samples were also labelled with an irrelevant pentamer. $\mathrm{CD} 8{ }^{+} \mathrm{D}^{\mathrm{b}}-\mathrm{NP}_{366-374^{+}}$specific $\mathrm{T}$ cells were observed in all tissues examined, spleen, mediastinal lymph node (MLN) and bronchoalveolar lavage (BAL) (Figure 3.3 A-D and Figure 3.4). In both the MLN and spleen the percentage and number of $C D 8^{+} \mathrm{D}^{\mathrm{b}}-\mathrm{NP}_{366-374^{+}}$ T cells did not significantly differ between the PKO and C57BL/6 mice. However, in the BAL, C57BL/6 mice showed a statistically significant higher percentage and number of $\mathrm{CD}^{+} \mathrm{D}^{\mathrm{b}}-\mathrm{NP}_{366-374}{ }^{+} \mathrm{T}$ cells compared to PKO mice (Figure $3.3 \mathrm{D}$ and Figure 3,4 C). Overall these data indicate that there was no difference between the two mouse strains in the frequency of $\mathrm{CD}^{+} \mathrm{D}^{\mathrm{b}}-\mathrm{NP}_{366-374}{ }^{+} \mathrm{T}$ cells in MLN and spleen, whereas in BAL the frequency of $\mathrm{CD}^{+} \mathrm{D}^{\mathrm{b}}-\mathrm{NP}_{366-374}{ }^{+} \mathrm{T}$ cells was decreased in $\mathrm{PKO}$ mice compared to C57BL/6 mice during a primary influenza infection.

Although the percentage of $\mathrm{CD} 8^{+} \mathrm{D}^{\mathrm{b}}-\mathrm{NP}_{366-374}{ }^{+} \mathrm{T}$ cells did not appear to be increased in PKO mice compared to C57BL/6 mice during a primary influenza infection, it was of interest to determine if the antigen-specific T cells in PKO mice had an activated phenotype and whether this was similar to the phenotype of cells from C57BL/6 mice. To assess this, cell surface expression of CD62L was analysed on antigen-specific $\mathrm{CD}^{+} \mathrm{T}$ cells. A decrease in CD62L expression is characteristic of effector cells. In the MLN, spleen and BAL, 80-100 \% of the $\mathrm{CD}^{+} \mathrm{D}^{\mathrm{b}}-\mathrm{NP}_{366-374}{ }^{+} \mathrm{T}$ cells expressed low levels of CD62L, demonstrating that they all had an effector phenotype (Figure 3.3 E). This effector phenotype was observed in both PKO and C57BL/6 mice indicating that the activation status of the $\mathrm{CD}^{+} \mathrm{D}^{\mathrm{b}}-\mathrm{NP}_{366-374}{ }^{+} \mathrm{T}$ cells between the two mouse strains, as determined by CD62L expression, was similar. 
Figure 3.3 C57BL/6 and PKO mice generate similar influenza-specific $\mathrm{CD8}^{+} \mathrm{T}$ cells responses after infection with $\mathrm{HKx} 31$.

C57BL/6 and PKO mice were infected i.n. with the HKx31 virus. BAL and tissues were harvested 10 days later and processed into single cell suspensions. MLN samples were digested in a solution of collagenase II and DNase I to obtain a single cell suspension. Cell suspensions were labelled with anti-CD8, anti-CD62 $\mathrm{L}$ and $\mathrm{D}^{\mathrm{b}}-\mathrm{NP}_{366-374}$ pentamer or an irrelevant pentamer $\left(\mathrm{D}^{\mathrm{b}}-\mathrm{GP}_{33-41}\right)$. The percentage of antigen-specific cells was determined by flow cytometry. Dead $\left(\mathrm{PI}^{+}\right)$cells were excluded and lymphocytes were gated on the basis of their FSC vs. SSC properties. (A) Panels show dot plots of spleen $\mathrm{CD} 8^{+}$cells, $\mathrm{D}^{\mathrm{b}}-\mathrm{NP}_{366-374}{ }^{+}$cells are highlighted by an oval. The percentages of $\mathrm{CD}^{+} \mathrm{D}^{\mathrm{b}}-\mathrm{NP}_{366-374}{ }^{+} \mathrm{T}$ cells from the indicated sources are shown in panels (B-D). (E) Percentages of $\mathrm{CD} 8^{+} \mathrm{D}^{\mathrm{b}}-\mathrm{NP}_{366-374}{ }^{+} \mathrm{CD} 62 \mathrm{~L}^{\text {lo }}$ cells in various tissues. Each dot represents an individual mouse and lines represent the mean. $* *: 0.001<p<0.01$, and ns: not significant by an unpaired Student's $t$ test. 
A.

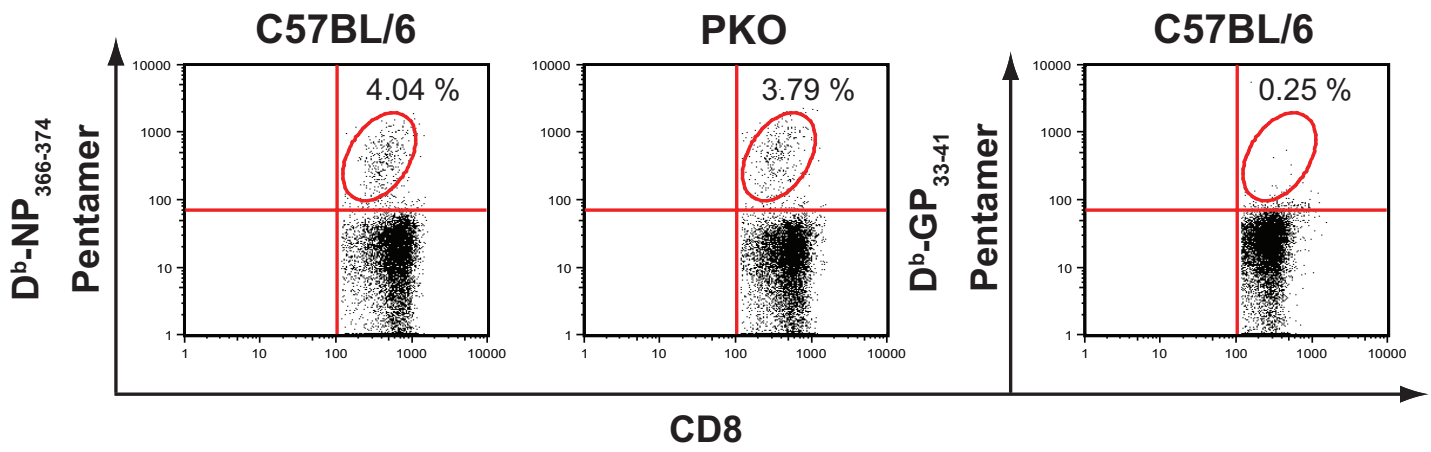

B.

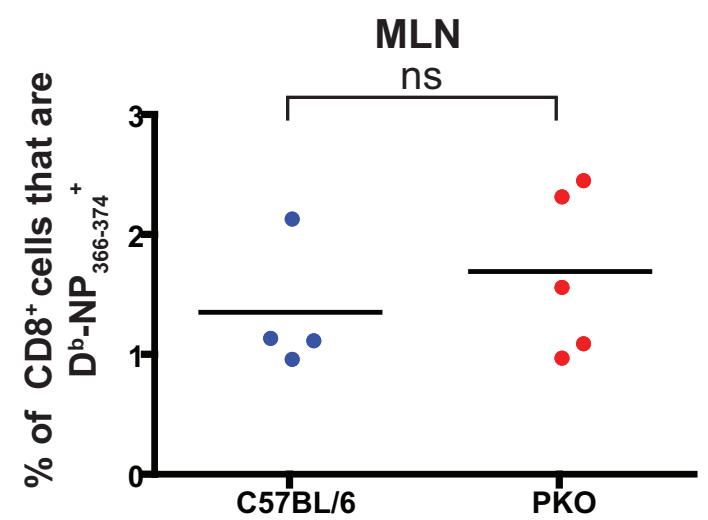

D.

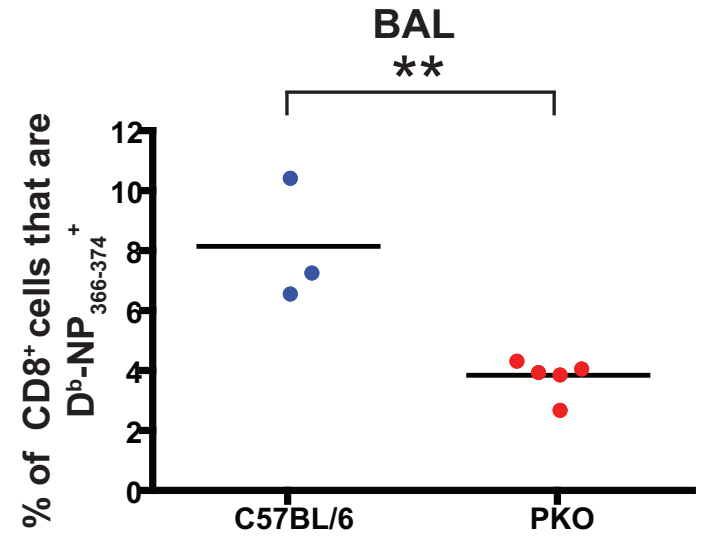

C.

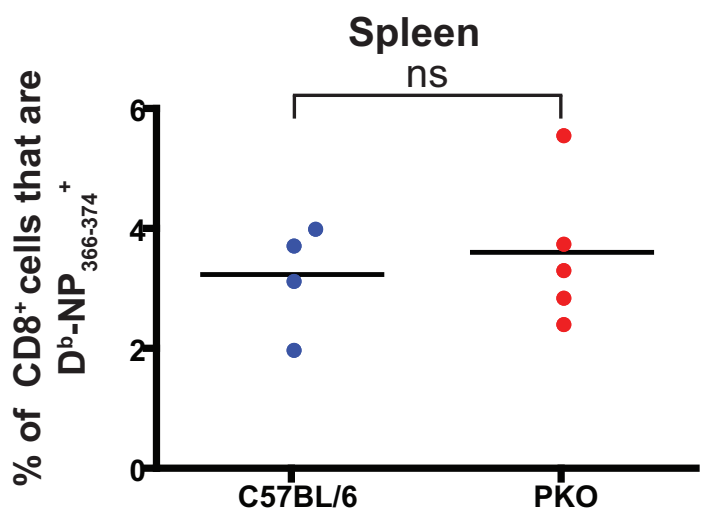

E.

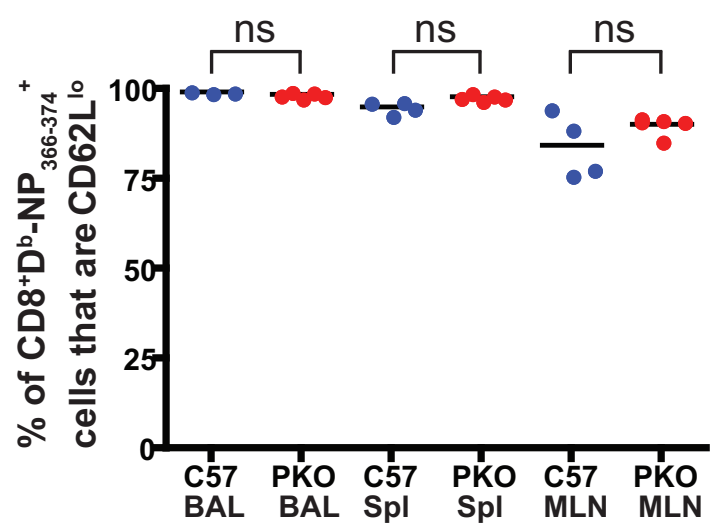


A.

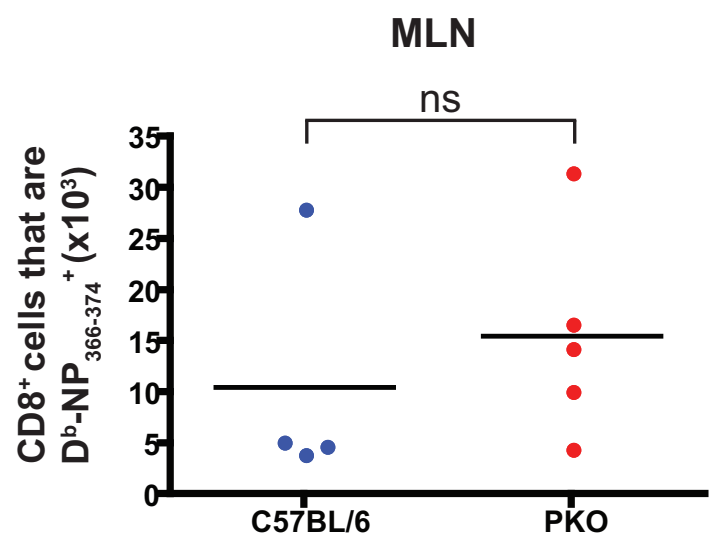

B.

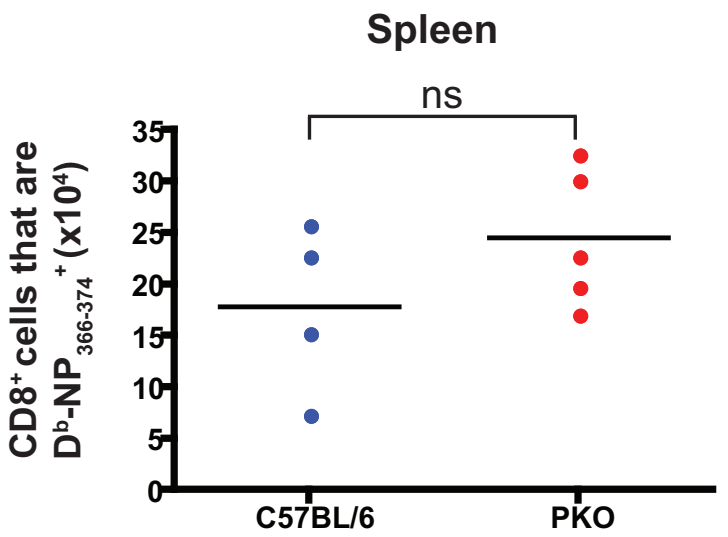

C.

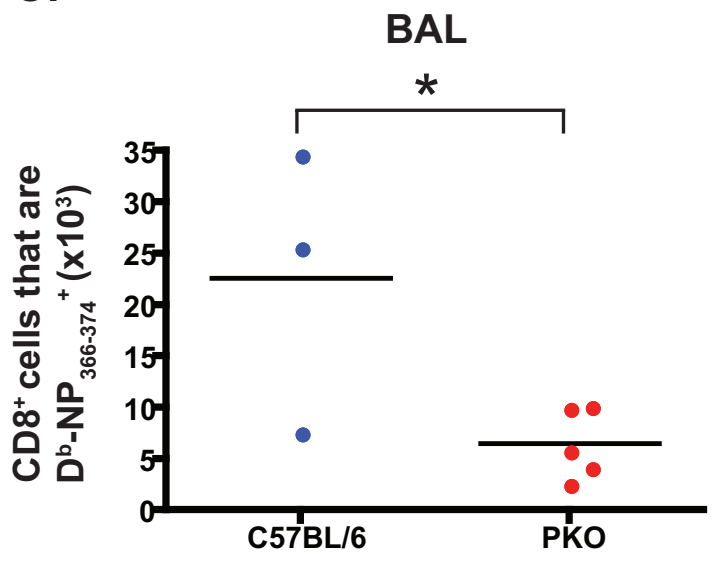

Figure 3.4 The number of $\mathrm{CD8}^{+} \mathrm{D}^{\mathrm{b}}-\mathrm{NP}_{366-374}{ }^{+} \mathrm{T}$ cells found in MLN and spleen is comparable between C57BL/6 and PKO mice after a primary infection with HKx31.

C57BL/6 and PKO mice were infected i.n. with the $\mathrm{HK} \times 31$ virus. BAL and tissues were harvested 10 days later and processed into single cell suspensions. MLN samples were digested in a solution of collagenase II and DNase I to obtain a single cell suspension. Cell suspensions were labelled with anti-CD8, anti-CD62L and $\mathrm{D}^{\mathrm{b}}-\mathrm{NP}_{366-374}$ pentamer or an irrelevant pentamer $\left(\mathrm{D}^{\mathrm{b}}-\mathrm{GP}_{33-41}\right)$. The percentage of antigen-specific cells was determined by flow cytometry. Dead $\left(\mathrm{PI}^{+}\right)$cells were excluded and lymphocytes were gated on the basis of their FSC vs. SSC properties. The numbers of $\mathrm{CD} 8^{+} \mathrm{D}^{\mathrm{b}}-\mathrm{NP}_{366-374}{ }^{+} \mathrm{T}$ cells from the indicated sources are shown in panels. Each dot represents an individual mouse and lines represent the mean. $*: p<0.05$, and ns: not significant by an unpaired Student's $t$ test. 
The results obtained in Figure 3.3 and Figure 3.4 are specific to the time point analysed, therefore it was important to confirm these results by characterising the influenza-specific $\mathrm{CD}^{+} \mathrm{T}$ cell response over the course of a primary infection in C57BL/6 and PKO mice. To do this a time course experiment that tracked the percentage of $\mathrm{CD} 8^{+} \mathrm{D}^{\mathrm{b}}-\mathrm{NP}_{366-374}{ }^{+} \mathrm{T}$ cells in the blood over time was performed (Figure 3.5). After a primary HKx31 i.n. infection the greatest percentage of $\mathrm{CD}^{+} \mathrm{D}^{\mathrm{b}}-\mathrm{NP}_{366-374}{ }^{+}$specific $\mathrm{T}$ cells was observed 11 days after infection. This corresponded to $5-10 \%$ of $\mathrm{CD}^{+} \mathrm{T}$ cells in the blood being specific for the NP epitope (Figure 3.5 A). Following the peak of the observed T cell response the percentage of $\mathrm{CD}^{+} \mathrm{D}^{\mathrm{b}}-\mathrm{NP}_{366-374}{ }^{+} \mathrm{T}$ cells declined dramatically to only $2.5 \%$ of $\mathrm{CD}^{+} \mathrm{T}$ cells being specific for the NP epitope by day17 after the infection. At 103 days after infection the percentage of $\mathrm{CD}^{+} \mathrm{D}^{\mathrm{b}}-\mathrm{NP}_{366-374}{ }^{+} \mathrm{T}$ cells was maintained at approximately $1 \%$ (Figure 3.5 A). Similar results were observed in both C57BL/6 and PKO mice. Thus these data failed to reveal differences in the percentage of $C D 8^{+} \mathrm{D}^{\mathrm{b}}$ $\mathrm{NP}_{366-374}{ }^{+} \mathrm{T}$ cells between $\mathrm{C} 57 \mathrm{~B} / 6$ and PKO mice during a primary influenza infection and is consistent with the data reported in Figure 3.3.

It was also of interest to determine if the phenotype of $\mathrm{CD}^{+} \mathrm{D}^{\mathrm{b}}-\mathrm{NP}_{366-374}{ }^{+} \mathrm{T}$ cells differed between PKO and C57BL/6 mice over the course of the primary influenza infection. As already seen at day $10,80-100 \%$ of the $\mathrm{CD}^{+} \mathrm{D}^{\mathrm{b}}-\mathrm{NP}_{366-374}{ }^{+} \mathrm{T}$ cells expressed a low level of CD62L throughout the course of the infection and no differences were seen between the PKO and C57BL/6 mice (Figure 3.5 B). Overall, the percentage and phenotype of the $\mathrm{CD}^{+} \mathrm{D}^{\mathrm{b}}-\mathrm{NP}_{366-374}{ }^{+}$specific $\mathrm{T}$ cells in the blood during a primary infection were similar in both the C57BL/6 and PKO mice. 


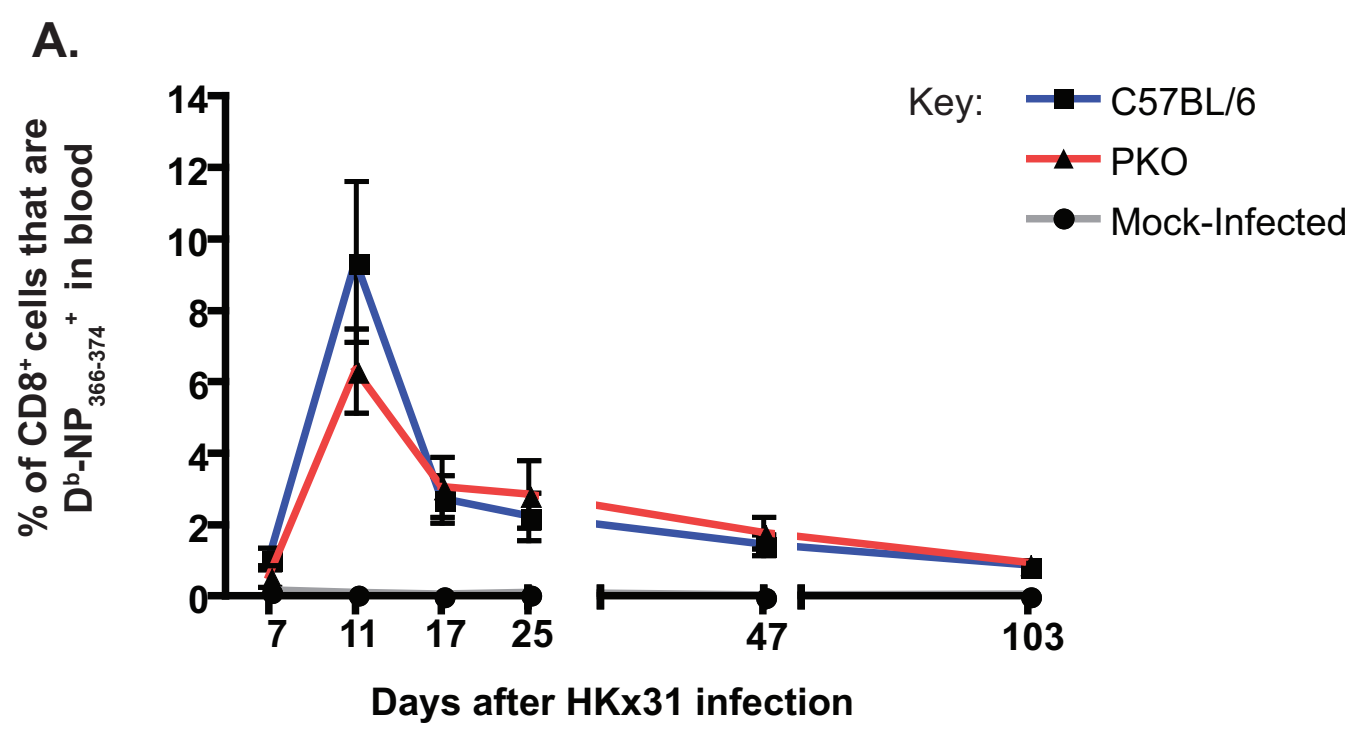

B.

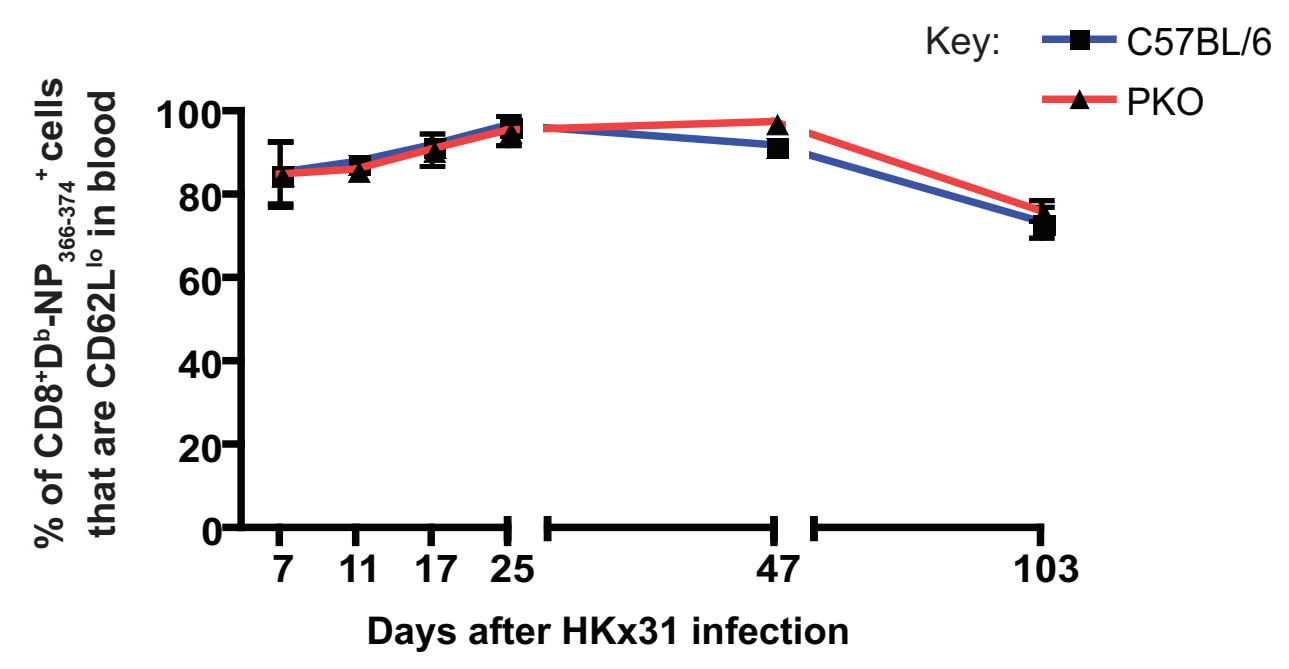

Figure 3.5 Similar percentages of $\mathrm{CD8}^{+} \mathrm{D}^{\mathrm{b}}-\mathrm{NP}_{366-374}{ }^{+} \mathrm{T}$ cells are found during an influenza infection in C57BL/6 and PKO mice.

C57BL/6 and PKO mice were infected i.n. with the HKx31 virus and blood was collected from the tail vein at specific time points after infection. Blood cells were labelled with anti-CD8, anti$\mathrm{CD} 62 \mathrm{~L}$ and $\mathrm{D}^{\mathrm{b}}-\mathrm{NP}_{366-374}$ pentamer. Blood from mock-infected mice was used as a negative control. Percentage of $\mathrm{CD}^{+} \mathrm{D}^{\mathrm{b}}-\mathrm{NP}_{366-374}{ }^{+}$cells was analysed by flow cytometry. Dead ( $\mathrm{PI}^{+}$) cells were excluded and then lymphocytes were gated on the basis of their FSC vs. SSC properties. Graphs show the percentage of $\mathrm{CD}^{+} \mathrm{D}^{\mathrm{b}}-\mathrm{NP}_{366-374}{ }^{+}$cells $(\mathrm{A})$ and $\mathrm{CD}^{+} \mathrm{D}^{\mathrm{b}}-\mathrm{NP}_{366-374}{ }^{+} \mathrm{CD} 62 \mathrm{~L}^{\text {lo }}$ cells (B) in the blood at specific times after a primary infection with the $\mathrm{HKx} 31$ virus. Each data point represents mean \pm SD of 3 mice per group. No statistical differences were found when the C57BL/6 and PKO infected mice were compared by a two-way ANOVA with a Bonferroni post test. These experiments were repeated twice with similar results. 
It has been reported that in $\mathrm{C} 57 \mathrm{BL} / 6$ mice the $\mathrm{D}^{\mathrm{b}}-\mathrm{NP}_{366-374}$ epitope is co-dominant with another epitope, $\mathrm{D}^{\mathrm{b}}-\mathrm{PA}_{224-233}$ during a primary infection $(354,355)$. Therefore it was important to determine if this co-dominance was maintained in the PKO mice. In a primary infection (Figure 3.6, left panels) the percentage of $\mathrm{CD}^{+} \mathrm{D}^{\mathrm{b}}-\mathrm{NP}_{366-374}{ }^{+}$and $\mathrm{CD} 8^{+} \mathrm{D}^{\mathrm{b}}-\mathrm{PA}_{224-}$ ${ }_{233}{ }^{+} \mathrm{T}$ cells was similar in both mouse strains. This confirms that PKO mice also exhibit co-dominance of the two $\mathrm{CD}^{+}$epitopes similar to what has previously been reported by other authors $(354,355)$. Taken together these data show that PKO mice do not have a greater percentage of antigen-specific $\mathrm{CD} 8^{+} \mathrm{T}$ cells compared to $\mathrm{C} 57 \mathrm{BL} / 6$ mice after a primary influenza A infection.

\subsubsection{Evaluation of the influenza-specific T cell response using MHC I pentamers in C57BL/6 and PKO mice during a secondary infection}

Investigations into the primary influenza immune response only assess the frequency of $\mathrm{CTL}$ and not memory $\mathrm{CD}^{+} \mathrm{T}$ cells. However, there is evidence to suggest that memory $\mathrm{CD}^{+} \mathrm{T}$ cells can also be involved in DC elimination $(169,344,345)$. To investigate whether perforin deficiency is associated with increased memory $\mathrm{CD}^{+} \mathrm{T}$ cells, a secondary influenza infection was established. Mice were first infected i.p. with the PR8 virus followed by an i.n. challenge with the $\mathrm{HKx} 31$ virus 30 days later. Both the $\mathrm{D}^{\mathrm{b}}-\mathrm{NP}_{366-}$ $374^{+}$and $\mathrm{D}^{\mathrm{b}}-\mathrm{PA}_{224-233}{ }^{+}$epitopes were investigated. It was found that both in C57BL/6 and PKO mice there was an increased percentage of $\mathrm{CD}^{+} \mathrm{D}^{\mathrm{b}}-\mathrm{NP}_{366-374}{ }^{+} \mathrm{T}$ cells compared to $\mathrm{CD}^{+} \mathrm{D}^{\mathrm{b}}-\mathrm{PA}_{224-233}{ }^{+} \mathrm{T}$ cells during the secondary response (Figure 3.5, middle panels and Figure 3.6 A). The frequency of antigen-specific T cells was highest 10 days after the secondary challenge in both the C57BL/6 and PKO mice (Figure $3.7 \mathrm{~A}$ ). In addition, over the course of the secondary infection, C57BL/6 mice maintained a statistically significant 3-fold higher percentage of $\mathrm{CD} 8^{+} \mathrm{D}^{\mathrm{b}}-\mathrm{NP}_{366-374}{ }^{+} \mathrm{T}$ cells in the blood compared to PKO mice (Figure $3.7 \mathrm{~A}$ ). In fact the percentage of $\mathrm{CD}^{+} \mathrm{D}^{\mathrm{b}}-\mathrm{NP}_{366-374}{ }^{+} \mathrm{T}$ cells in $\mathrm{PKO}$ mice during a secondary infection did not significantly increase compared to the primary response (Figure $3.7 \mathrm{~A}$ ). No difference was observed in the percentage of $\mathrm{CD} 8^{+} \mathrm{D}^{\mathrm{b}}-\mathrm{PA}_{224-233}{ }^{+} \mathrm{T}$ cells between the two mouse strains (Figure 3.7 B). Furthermore, at the height of the observed response, day 10 , the percentage of $\mathrm{CD} 8^{+} \mathrm{D}^{\mathrm{b}}-\mathrm{PA}_{224-233}{ }^{+} \mathrm{T}$ cells did not differ between the primary and secondary influenza infections (Figure 3.7 B). Overall, these data indicate that during a secondary influenza infection the frequency of $\mathrm{CD} 8^{+} \mathrm{D}^{\mathrm{b}}-\mathrm{PA}_{224-233}{ }^{+} \mathrm{T}$ cells was similar in PKO and C57BL/6 mice, while a markedly reduced percentage of $\mathrm{CD}^{+} \mathrm{D}^{\mathrm{b}}$ $\mathrm{NP}_{366-374}{ }^{+} \mathrm{T}$ cells was observed in PKO mice compared to C57BL/6 mice. 
The effector phenotype of $\mathrm{CD} 8^{+} \mathrm{D}^{\mathrm{b}}-\mathrm{NP}_{366-374}{ }^{+} \mathrm{T}$ cells during the secondary infection, was determined by measuring the cell surface expression of CD62L by flow cytometry. As in the primary influenza infection, 80-100\% of the $\mathrm{CD}^{+} \mathrm{D}^{\mathrm{b}}-\mathrm{NP}_{366-374}{ }^{+} \mathrm{T}$ cells expressed low levels of CD62L (Figure 3.7 C). This was observed in both C57BL/6 and PKO mice throughout the course of the secondary infection (Figure 3.7 C). So, although the percentage of $\mathrm{CD}^{+} \mathrm{D}^{\mathrm{b}}-\mathrm{NP}_{366-374}{ }^{+} \mathrm{T}$ cells was lower in PKO mice compared to C57BL/6 mice the cells that were present were activated and expressed an effector phenotype similar to that seen in $\mathrm{C} 57 \mathrm{BL} / 6$ mice.

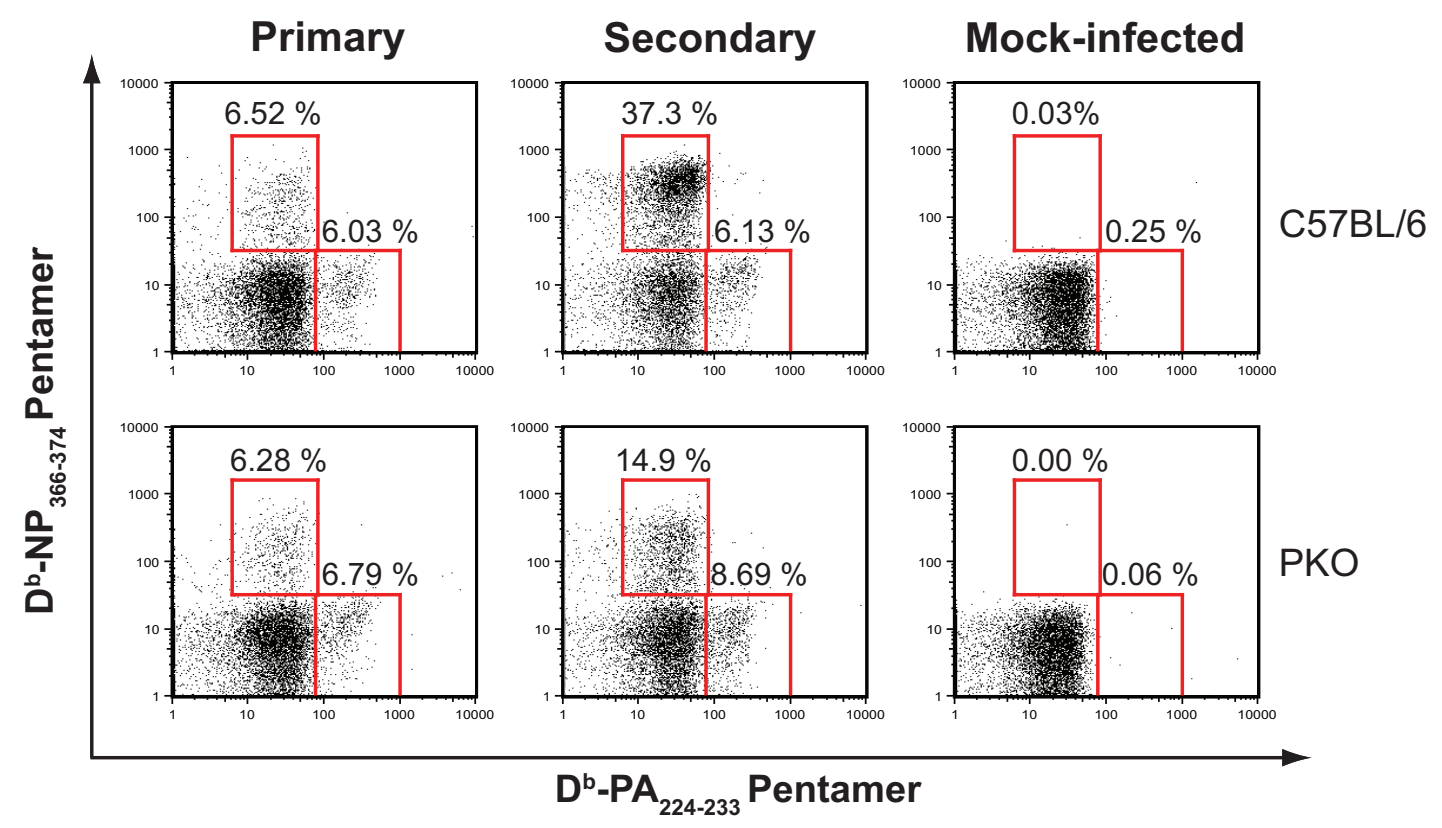

Figure 3.6 Detection of $\mathrm{D}^{\mathrm{b}}-\mathrm{NP}_{366-374}{ }^{+}$and $\mathrm{D}^{\mathrm{b}}-\mathrm{PA}_{224-233} \mathrm{CD8}^{+} \mathrm{T}$ cells during a primary and a secondary influenza infection.

C57BL/6 and PKO mice were infected i.n. with the HKx31 virus (Primary) or were infected i.p. with the PR8 virus and 30 days later infected i.n. with $\mathrm{HKx} 31$ virus (Secondary). As a negative control mice were mock-infected with PBS. Blood was collected 10 days later from the tail vein and cells were labelled with anti-CD8, $\mathrm{D}^{\mathrm{b}}-\mathrm{NP}_{366-374}$ and $\mathrm{D}^{\mathrm{b}}-\mathrm{PA}_{224-233}$ pentamers. Percentage of antigen-specific cells was determined by flow cytometry. Dead $\left(\mathrm{PI}^{+}\right)$cells were excluded and then lymphocytes were gated on the basis of their FSC vs. SSC properties. Panels show representative dot plots of blood CD $8^{+}$cells, $\mathrm{D}^{\mathrm{b}}-\mathrm{NP}_{366-374}{ }^{+}$and $\mathrm{D}^{\mathrm{b}}-\mathrm{PA}_{224-233^{+}}{ }^{+}$cells are highlighted by rectangle gates. 


\section{Figure 3.7 A reduced percentage of $\mathrm{CD8}^{+} \mathrm{D}^{\mathrm{b}}-\mathrm{NP}_{366-374}{ }^{+} \mathrm{T}$ cells is observed in $\mathrm{PKO}$ mice compared to $\mathrm{C} 57 \mathrm{BL} / 6$ mice during a secondary influenza infection.}

C57BL/6 and PKO mice were infected i.n. with the HKx31 virus (Primary) or were infected i.p. with the PR8 virus and 30 days later infected i.n. with HKx31 virus (Secondary). As a negative control mice were mock-infected with PBS. At specific time points after infection blood was collected from the tail vein. Blood was labelled with anti-CD8, $\mathrm{D}^{\mathrm{b}}-\mathrm{NP}_{366-374}$ and $\mathrm{D}^{\mathrm{b}}-\mathrm{PA}_{224-233}$ pentamers. The percentage of antigen-specific cells was determined by flow cytometry. Dead $\left(\mathrm{PI}^{+}\right)$ cells were excluded and lymphocytes were gated on the basis of their FSC vs. SSC properties. The percentage of $\mathrm{CD} 8^{+} \mathrm{D}^{\mathrm{b}}-\mathrm{NP}_{366-374}{ }^{+}$cells $(\mathrm{A}), \mathrm{CD} 8^{+} \mathrm{D}^{\mathrm{b}}-\mathrm{PA}_{224-233}{ }^{+}$cells $(\mathrm{B})$ and $\mathrm{CD} 8^{+} \mathrm{D}^{\mathrm{b}}-\mathrm{NP}_{366-374}{ }^{+}$ CD62 $\mathrm{L}^{\text {lo }}$ cells $(\mathrm{C})$ in the blood was shown at specific time points after primary and secondary influenza infection. Each data point represents mean \pm SD of 3 mice per group. The statistical analysis compares values for the C57BL/6 and PKO secondary infected mice. $* * *: p<0.001$ by a two-way ANOVA with a Bonferroni post test. Experiment A, has been repeated twice with similar results. 
A.

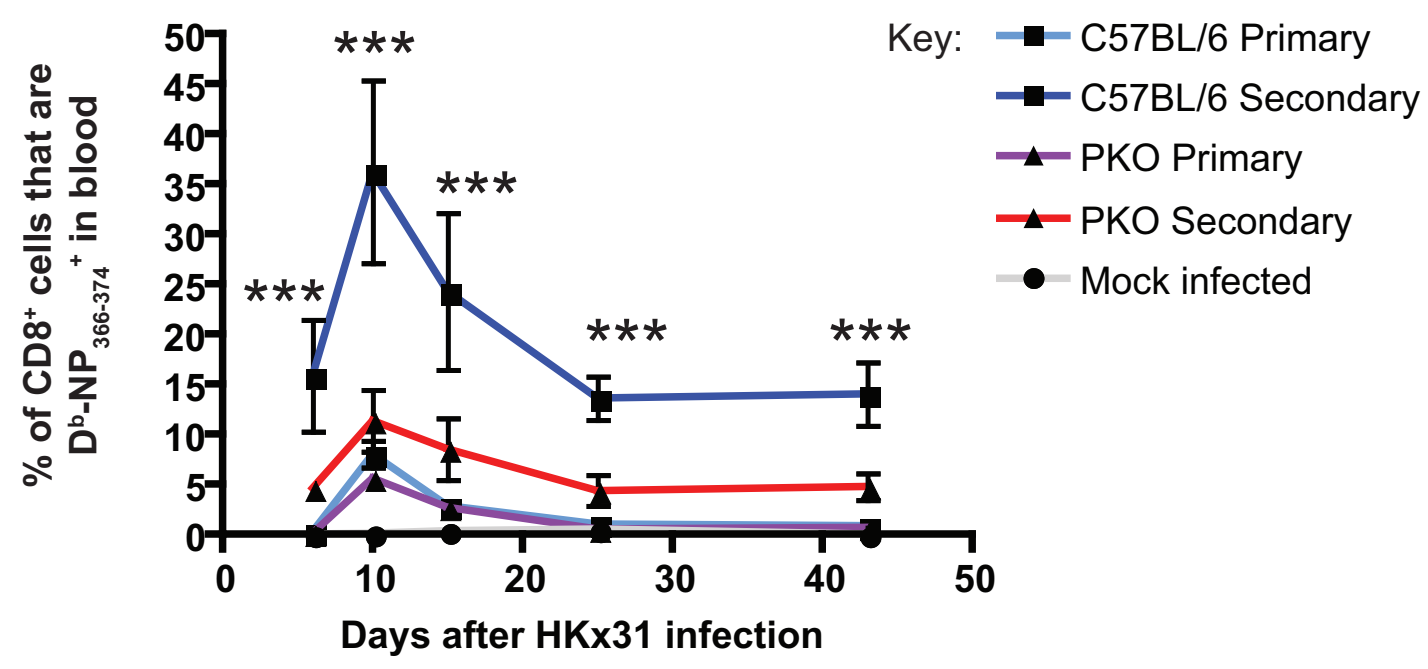

B.

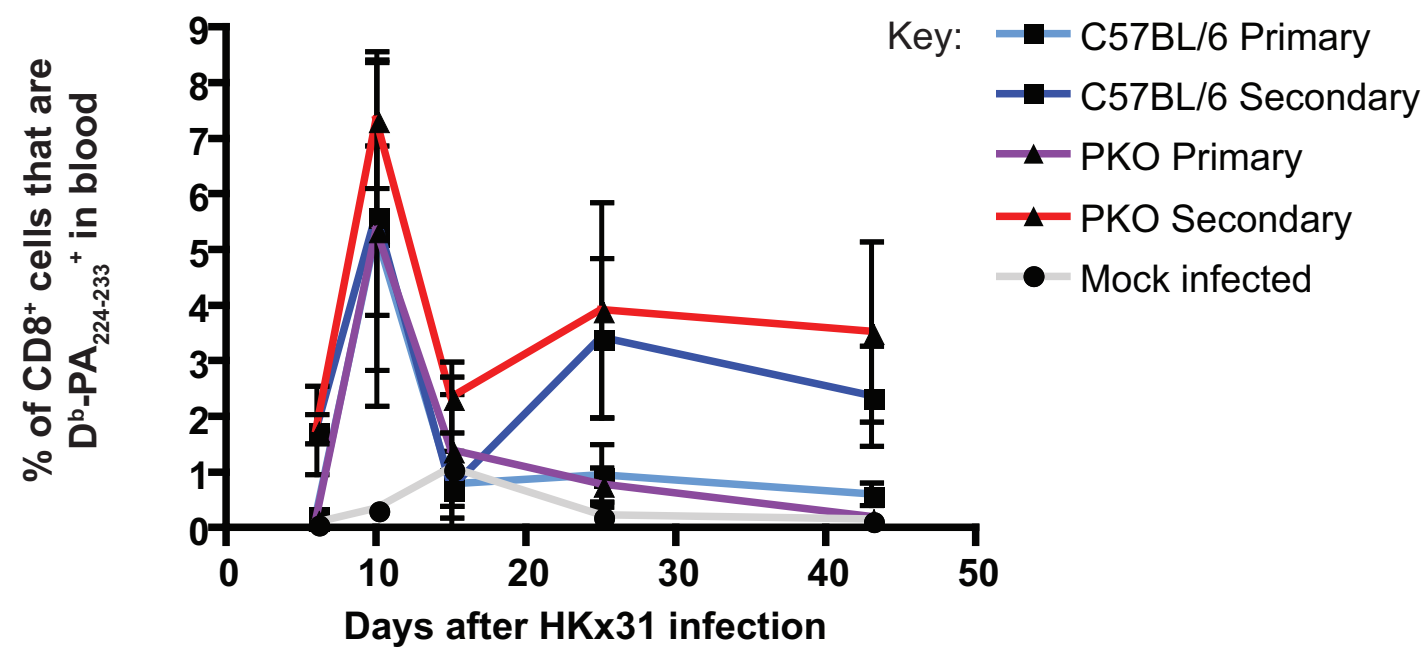

C.

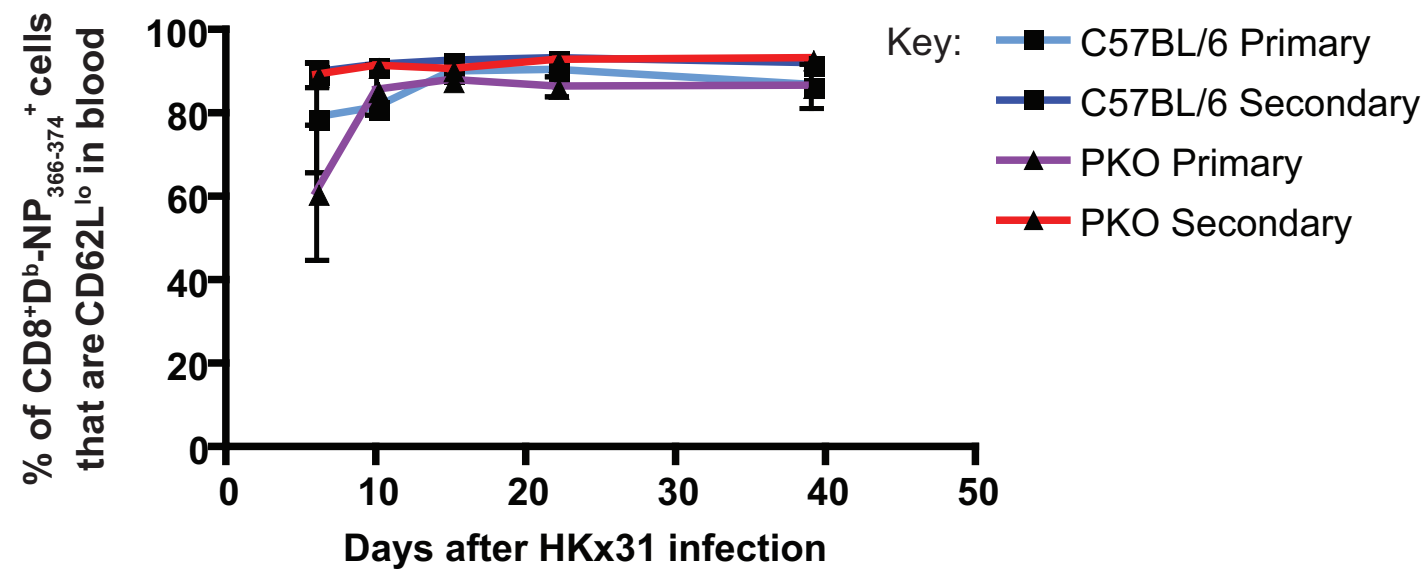




\subsubsection{Evaluation of the influenza-specific $T$ cell response by measuring the production of specific IFN- $\gamma$ in C57BL/6 and PKO mice}

To further assess the influenza-specific immune response the number of antigen-specific $\mathrm{CD}^{+} \mathrm{T}$ cells was determined by calculating the number of cells producing IFN- $\gamma$ after restimulation with a specific peptide. This method was commonly used before the availability of MHC I pentamers to evaluate the number of antigen-specific $\mathrm{CD} 8^{+} \mathrm{T}$ cells. This detection method of intracellular IFN- $\gamma$ provides information not only about the cell number but also their effector function. To determine the number of influenza-specific $\mathrm{CD}^{+} \mathrm{T}$ cells producing IFN- $\gamma$, single cell suspensions from various tissues were restimulated for 4-6 hr with either the $\mathrm{NP}_{366-374}$ or $\mathrm{PA}_{224-233}$ peptide. Cells were then labelled with anti-CD8 and anti-IFN- $\gamma$ mAbs. To control for non-specific intracellular antibody binding, control samples were labelled with an isotype control antibody.

Initially, BAL cells were collected 13 days after primary infection with the HKx31 virus and restimulated with $\mathrm{NP}_{366-374}$ peptide (Figure 3.8 A). PKO mice had a slight increase in the percentage of $\mathrm{NP}_{366-374}$ responsive cells producing IFN- $\gamma ; 4.7 \%$ compared with $3.2 \%$ in the C57BL/6 mice. However, as pooled samples were used due to the low cell count in the BAL no statistical analysis could be carried out to determine if this difference was statistically significant. Next the percentage of $\mathrm{NP}_{366-374}$ responsive T cells producing IFN$\gamma$ in the spleen and MLN 11 days after a primary infection was investigated. No statistical difference was seen in the frequency of $\mathrm{NP}_{366-374}$ responsive $\mathrm{T}$ cells producing IFN- $\gamma$ in the PKO or C57BL/6 mice in either the MLN or spleen (Figure 3.8 B and Figure 3.9 A and B). This result correlated with the data in Figure 3.3 B and C and Figure 3.4 A and B, where no difference was seen in the percentage of $\mathrm{CD} 8^{+} \mathrm{D}^{\mathrm{b}}-\mathrm{NP}_{366-374}{ }^{+} \mathrm{T}$ cells using the MHC I pentamer, and confirms previously published data showing that these two techniques produce comparable results $(177,354,372)$. Furthermore, these data indicate that the $\mathrm{CD}^{+} \mathrm{D}^{\mathrm{b}}-\mathrm{NP}_{366-374}{ }^{+} \mathrm{T}$ cells in both the $\mathrm{C} 57 \mathrm{BL} / 6$ and $\mathrm{PKO}$ mice have a similar effector function. 
Figure 3.8 Detection of intracellular IFN- $\gamma$ in $\mathrm{NP}_{366-374}{ }^{+}$responsive $\mathrm{T}$ cells in different tissues from C57BL/6 and PKO mice undergoing a primary influenza infection.

C57BL/6 and PKO mice were infected i.n. with the HKx31 virus. Thirteen (A) or 11 days (B) after influenza infection tissues were collected and processed. MLN samples were digested in a solution of collagenase II and DNase I to obtain a single cell suspension. Cell suspensions were then restimulated for $4 \mathrm{hr}$ with $1 \mu \mathrm{M} \mathrm{NP}_{366-374}$ peptide. Cells were labelled with anti-CD8 mAb, fixed and labelled intracellularly with anti-IFN- $\gamma$ mAb or with a rat IgG1 isotype matched control antibody and examined by flow cytometry. Lymphocytes were gated on the basis of their FSC vs. SSC properties. Dot plots show the percentage of BAL (A), MLN and spleen (B) CD8 ${ }^{+}$IFN- $\gamma$ producing cells in cultures stimulated with $\mathrm{NP}_{366-374}$ peptide. 
A.
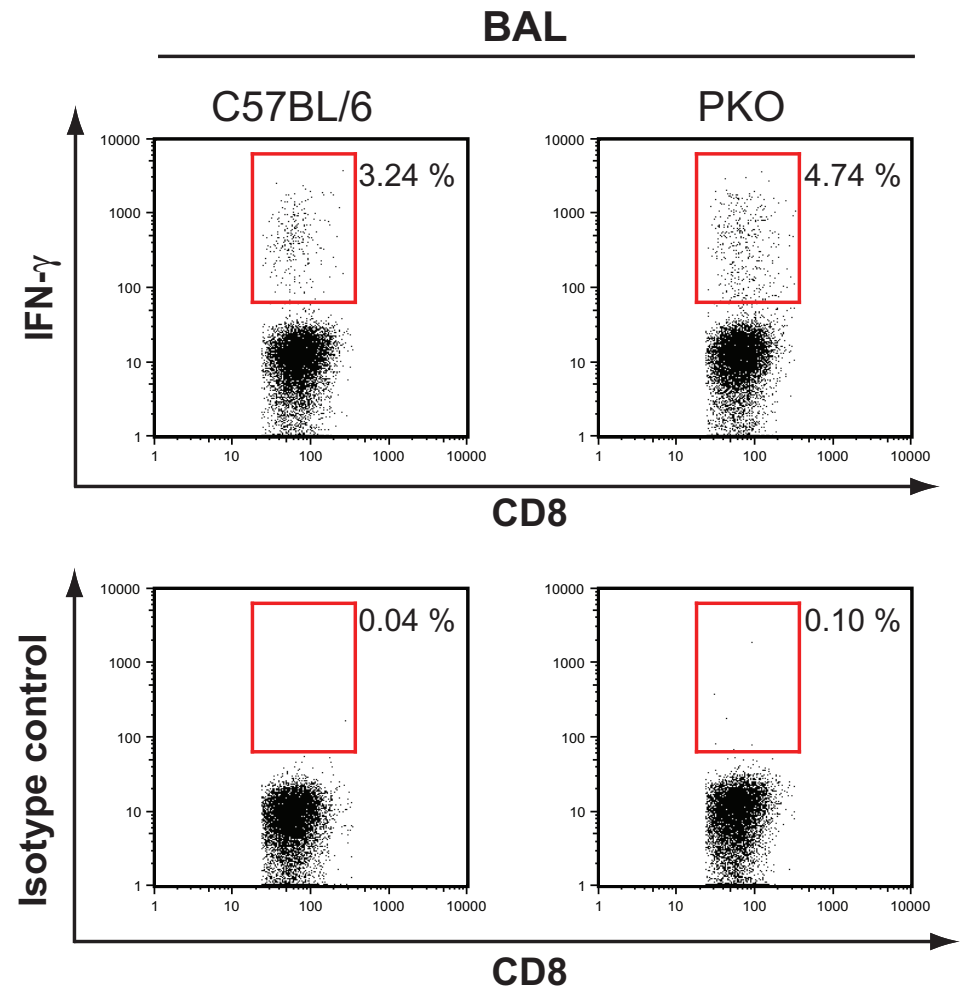

B.

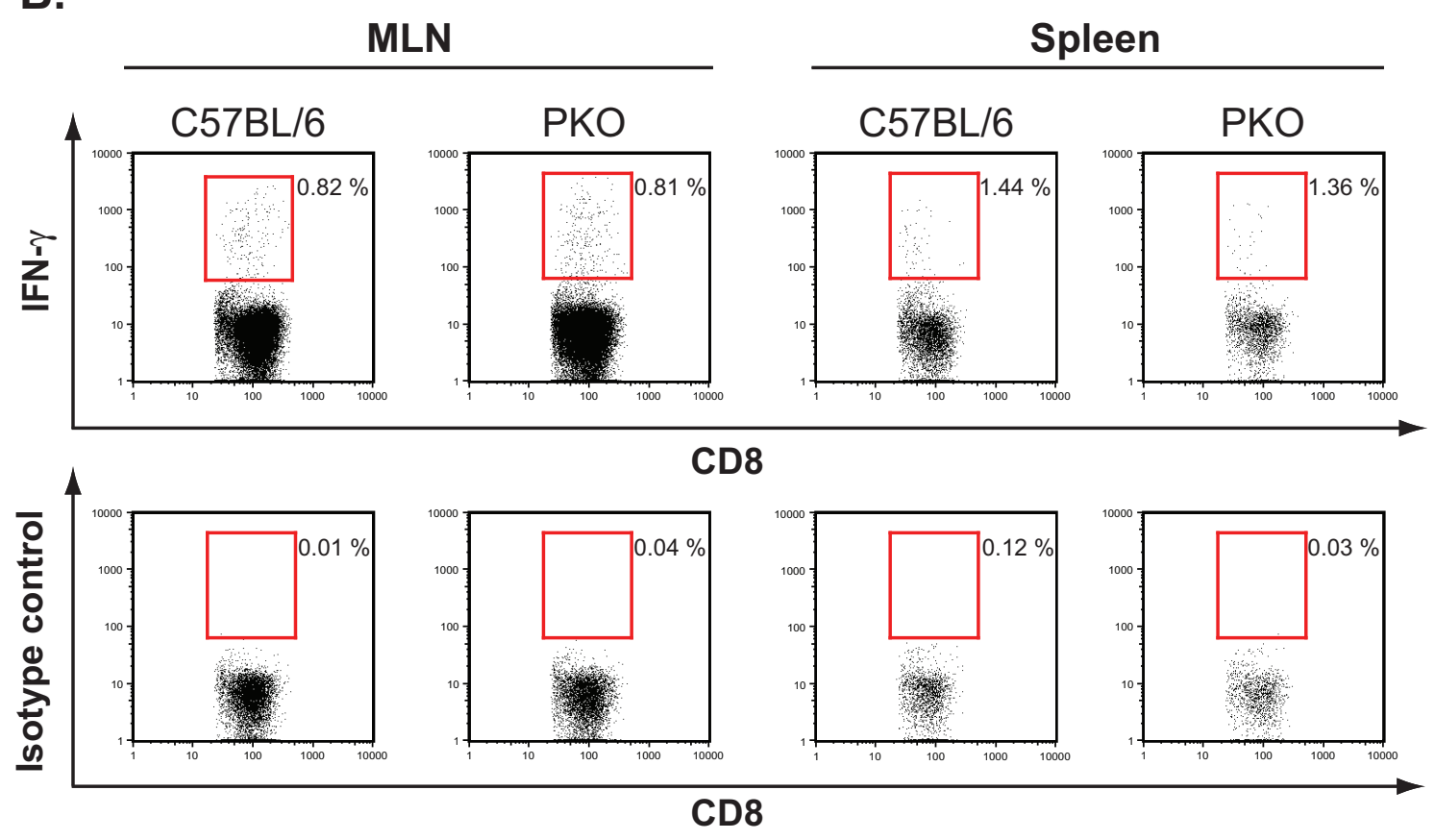


It was next asked whether the number of $\mathrm{CD}^{+} \mathrm{T}$ cells producing IFN- $\gamma$ after restimulation with the $\mathrm{NP}_{366-374}$ peptide was similar in the spleen and MLN between the two mouse strains over the course of a primary infection (Figure 3.9 A and B). Barely detectable numbers of $\mathrm{NP}_{366-374}$ responsive $\mathrm{T}$ cells were present five days after the primary infection, in the spleen and MLN of both C57BL/6 and PKO mice. Eight days after the primary infection, it was observed that the PKO mice had approximately 2-fold less $\mathrm{NP}_{366-374}$ responsive $\mathrm{T}$ cells producing IFN- $\gamma$ compared to $\mathrm{C} 57 \mathrm{BL} / 6$ mice in both the spleen and MLN. However, this difference was not found to be statistically significant (Figure 3.9 A and B). Eleven and 14 days after infection PKO and C57BL/6 mice had a similar level of $\mathrm{NP}_{366-374}$ responsive $\mathrm{T}$ cells producing IFN- $\gamma$. Taken together these data indicate that during a primary influenza infection the number of $\mathrm{NP}_{366-374}$ responsive $\mathrm{T}$ cells producing IFN- $\gamma$ in the MLN and spleen was similar in C57BL/6 and PKO mice.

It was also of interest to determine the number of $\mathrm{PA}_{224-233}$ responsive $\mathrm{T}$ cells producing IFN- $\gamma$ in the MLN and spleen at specific time points during a primary infection and verify the results found in the blood that were obtained using the MHC I pentamer. The number of $\mathrm{PA}_{224-233}$ responsive T cells producing IFN- $\gamma$ in the MLN or spleen did not statistically differ between C57BL/6 and PKO mice at either 8 or 15 days after a primary influenza infection (Figure 3.9 C-F). This correlates with the data obtained using the MHC I pentamer (Figure 3.6). Overall, during a primary influenza infection there was no difference in the number or effector phenotype of the influenza-specific $\mathrm{CD} 8^{+} \mathrm{T}$ cells in C57BL/6 and PKO mice. 
Figure 3.9 A similar number of influenza-specific $\mathrm{T}$ cells produce IFN- $\gamma$ over the course of a primary infection in C57BL/6 and PKO mice

C57BL/6 and PKO mice were infected i.n. with the HKx31 virus. At specific time points post infection (p.i.) the MLN (A, C and D) and spleen (B, E and F) were collected and processed. MLN samples were digested in a solution of collagenase II and DNase I to obtain a single cell suspension. Cell suspensions were restimulated for $4 \mathrm{hr}$ with $1 \mu \mathrm{M} \mathrm{NP}{ }_{366-374}\left(\mathrm{~A}\right.$ and $\mathrm{B}$ ) or $\mathrm{PA}_{224-233}$ (C - F) peptide. Cells were labelled with anti-CD8 mAb, fixed and labelled intracellularly with anti-IFN- $\gamma \mathrm{mAb}$ and examined by flow cytometry. Lymphocytes were gated on the basis of their FSC vs. SSC properties. Each data point represents mean \pm SD of 3 mice per group. (A and B) No statistical difference found between infected C57BL/6 and PKO mice using a two-way ANOVA with a Bonferroni post test. (C - F) ns: not significant by a one-way ANOVA with a Tukey post test. 
A.

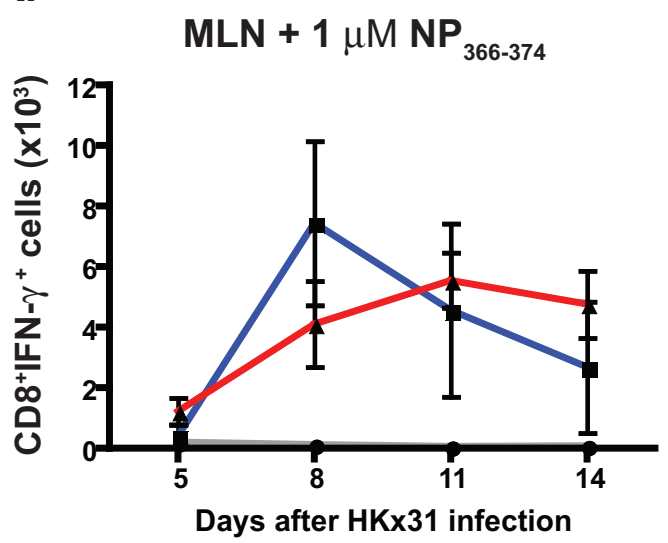

B.

Spleen $+1 \mu \mathrm{M} \mathrm{NP}_{366-374}$

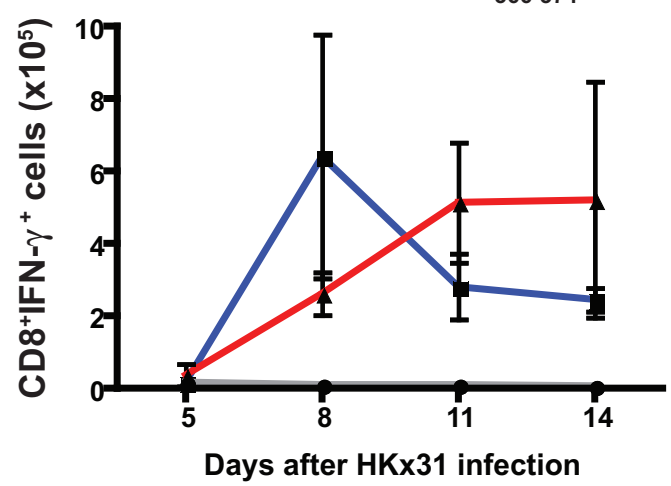

$$
\begin{aligned}
\text { Key: } & \rightarrow \text { C57BL/6 } \\
& - \text { PKO } \\
& \bullet-\text { Mock-infected }
\end{aligned}
$$

C.

MLN Day 8 p.i. +

$1 \mu \mathrm{M} \mathrm{PA}_{224-233}$

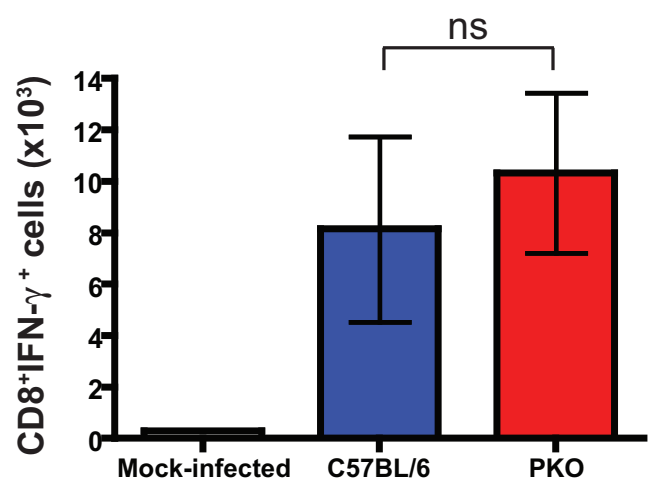

E.

Spleen Day 8 p.i. +

$1 \mu \mathrm{M} \mathrm{PA}_{224-233}$

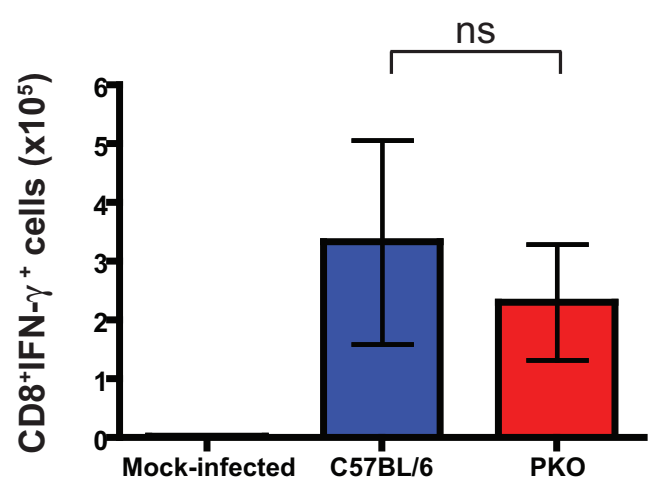

D.

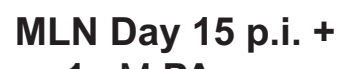

$1 \mu \mathrm{M} \mathrm{PA}_{224-233}$

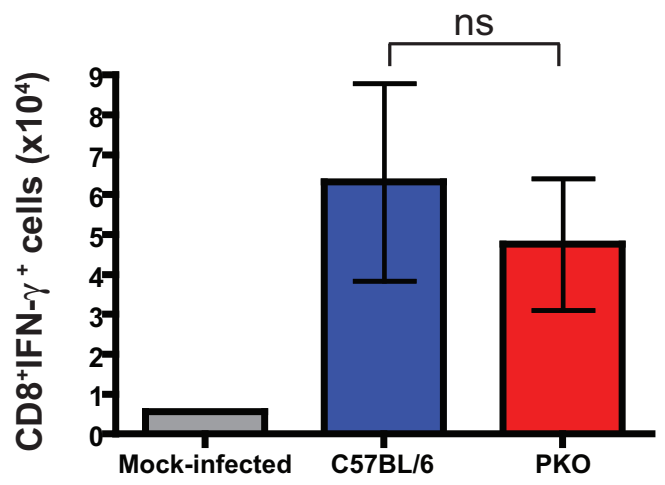

F.

Spleen Day 15 p.i. +

$1 \mu \mathrm{M} \mathrm{PA}_{224-233}$

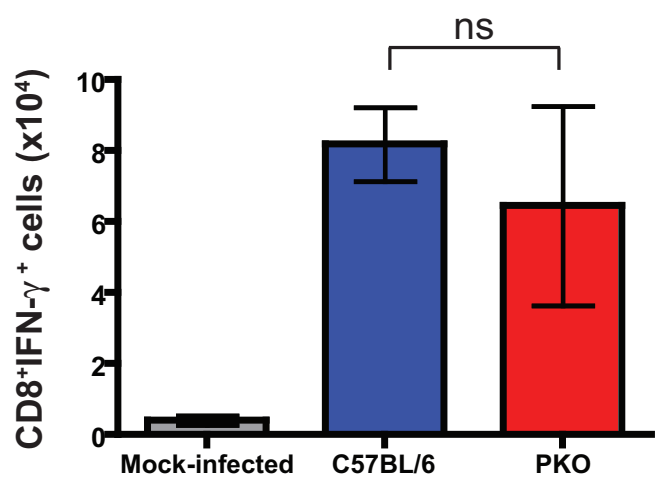


The number of antigen specific $\mathrm{CD} 8^{+} \mathrm{T}$ cells producing IFN- $\gamma$ after restimulation with the $\mathrm{NP}_{366-374}$ and $\mathrm{PA}_{224-233}$ peptides was examined during a secondary influenza infection. Six days after the secondary challenge the number of $\mathrm{NP}_{366-374}$ responsive $\mathrm{T}$ cells was statistically greater in the MLN but statistically lower in the spleen of C57BL/6 mice compared to PKO mice (Figure 3.10 A and Figure 3.11 A). However, these statistical differences in the number of $\mathrm{NP}_{366-374}$ responsive $\mathrm{T}$ cells between the two mouse strains in the MLN and spleen were no longer observed 10 days after secondary challenge (Figure $3.10 \mathrm{~B}$ and Figure $3.11 \mathrm{~B}$ ). These results differ from the data obtained from the blood using the MHC I pentamer, where C57BL/6 mice had a statistically significant increase in the percentage of $\mathrm{CD}^{+} \mathrm{D}^{\mathrm{b}}-\mathrm{NP}_{366-374}{ }^{+} \mathrm{T}$ cells during the course of a secondary influenza infection (Figure $3.7 \mathrm{~A}$ ). Thus it appears that during a secondary influenza infection the number of $\mathrm{NP}_{366-374}$ responsive $\mathrm{T}$ cells varied between $\mathrm{C} 57 \mathrm{BL} / 6$ and $\mathrm{PKO}$ mice, but this was not consistent between different tissues.

The $\mathrm{D}^{\mathrm{b}}-\mathrm{PA}_{224-233}$ epitope is subdominant to the $\mathrm{D}^{\mathrm{b}}-\mathrm{NP}_{366-374}$ epitope during a secondary infection and unlike the $\mathrm{D}^{\mathrm{b}}-\mathrm{NP}_{366-374}$ epitope a similar number of $\mathrm{CD} 8^{+} \mathrm{D}^{\mathrm{b}}-\mathrm{PA}_{224-233}{ }^{+} \mathrm{T}$ cells are seen during both a primary and secondary infection $(354,355,357)$. The data obtained using the MHC I pentamer (Figure 3.7), confirmed these previously published data. Therefore it was next asked whether the technique of detecting antigen-specific $\mathrm{CD} 8^{+}$ T cells by intracellular IFN- $\gamma$ production would also reveal results similar to those seen in Figure 3.7, where the MHC I pentamer was used to detect the antigen-specific T cells during a secondary influenza infection. No statistical difference in the number of $\mathrm{PA}_{224-233}$ responsive T cells in either the spleen or MLN was found between C57BL/6 and PKO mice during a secondary influenza infection (Figure 3.10 and Figure 3.11). These results obtained using the IFN- $\gamma$ assay were consistent with the data acquired using the MHC I pentamer (Figure 3.7). Thus it appears that during a secondary influenza infection the number of $\mathrm{PA}_{224-233}$ responsive $\mathrm{T}$ cells was comparable between different tissues and did not differ between C57BL/6 and PKO mice. 
A.
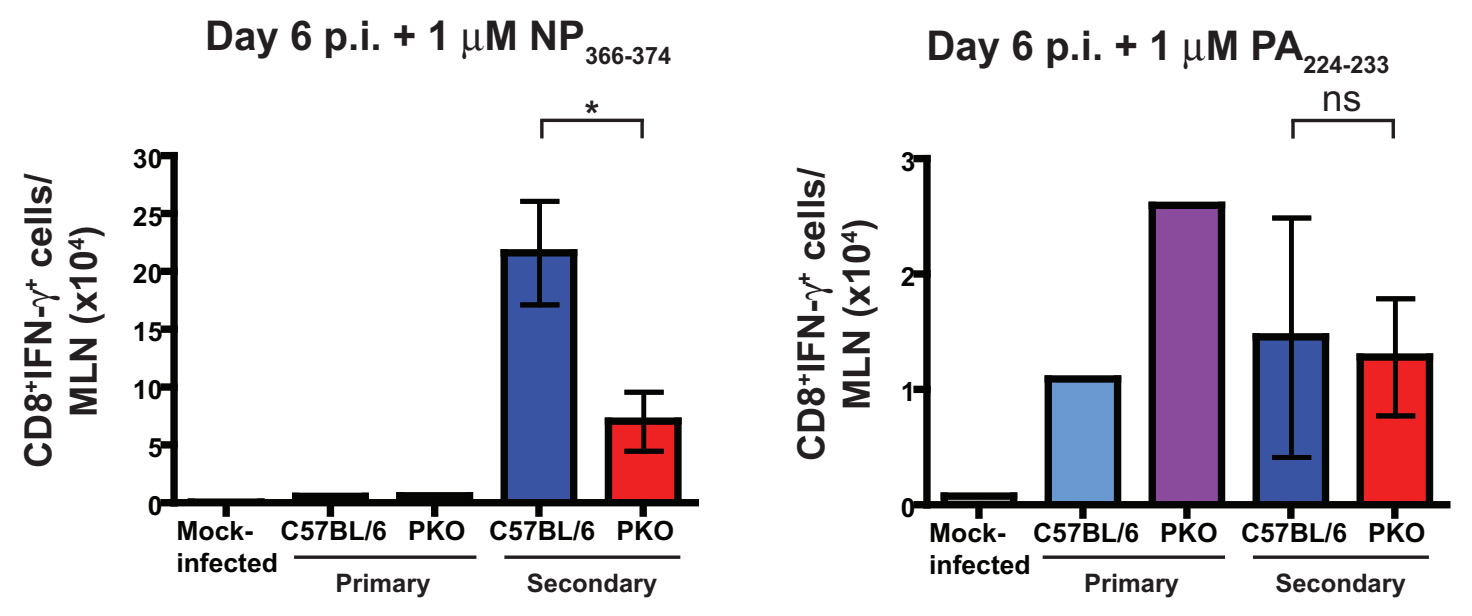

B.
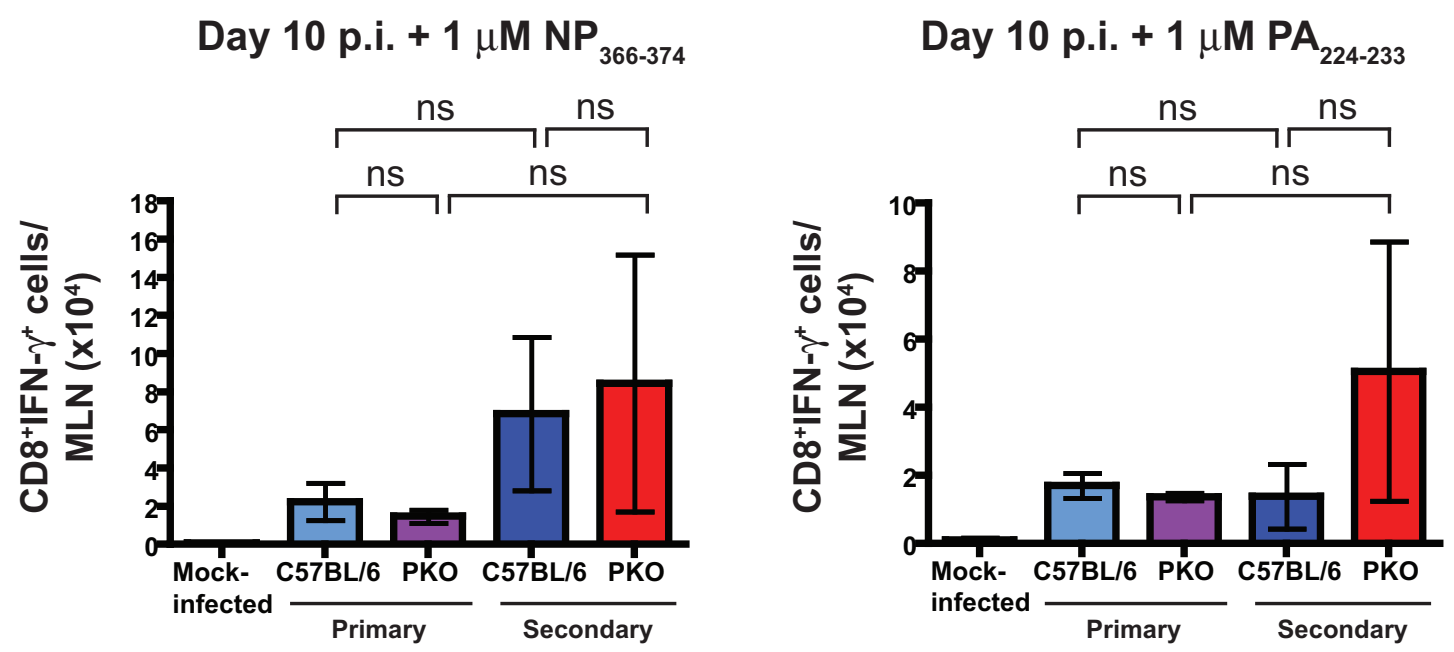

Figure 3.10 The number of antigen-specific $\mathrm{CD8}^{+} \mathrm{T}$ cells producing IFN- $\gamma$ in the MLN after a secondary influenza challenge.

C57BL/6 and PKO mice were infected i.n. with the HKx31 virus (Primary) or were infected i.p. with the PR8 virus and 30 days later infected i.n. with HKx31 virus (Secondary). As a negative control mice were mock-infected with PBS. At day 6 (A) and day 10 (B) after HKx31 virus infection the MLN were collected and processed. MLN samples were digested in a solution of collagenase II and DNase I to obtain a single cell suspension. Cell suspensions were restimulated for $4 \mathrm{hr}$ with $1 \mu \mathrm{M} \mathrm{NP}{ }_{366-374}$ or $\mathrm{PA}_{224-233}$ peptide as indicated. Cells were labelled with anti-CD8 $\mathrm{mAb}$, fixed and labelled intracellularly with anti-IFN- $\gamma \mathrm{mAb}$ and examined by flow cytometry. Lymphocytes were gated on the basis of their FSC vs. SSC properties. (A) The primary and mockinfected mice are pooled samples and each bar represents the data point, whereas in the secondary infected groups each bar represents mean \pm SD or range of 2- 3 mice per group. (B) Each bar represents mean \pm SD of 3 mice per group. (A) $*: p<0.05$ and ns: not significant by a Student's $t$ test. (B) ns: not significant by two-way ANOVA with a Tukey post test. 
A.
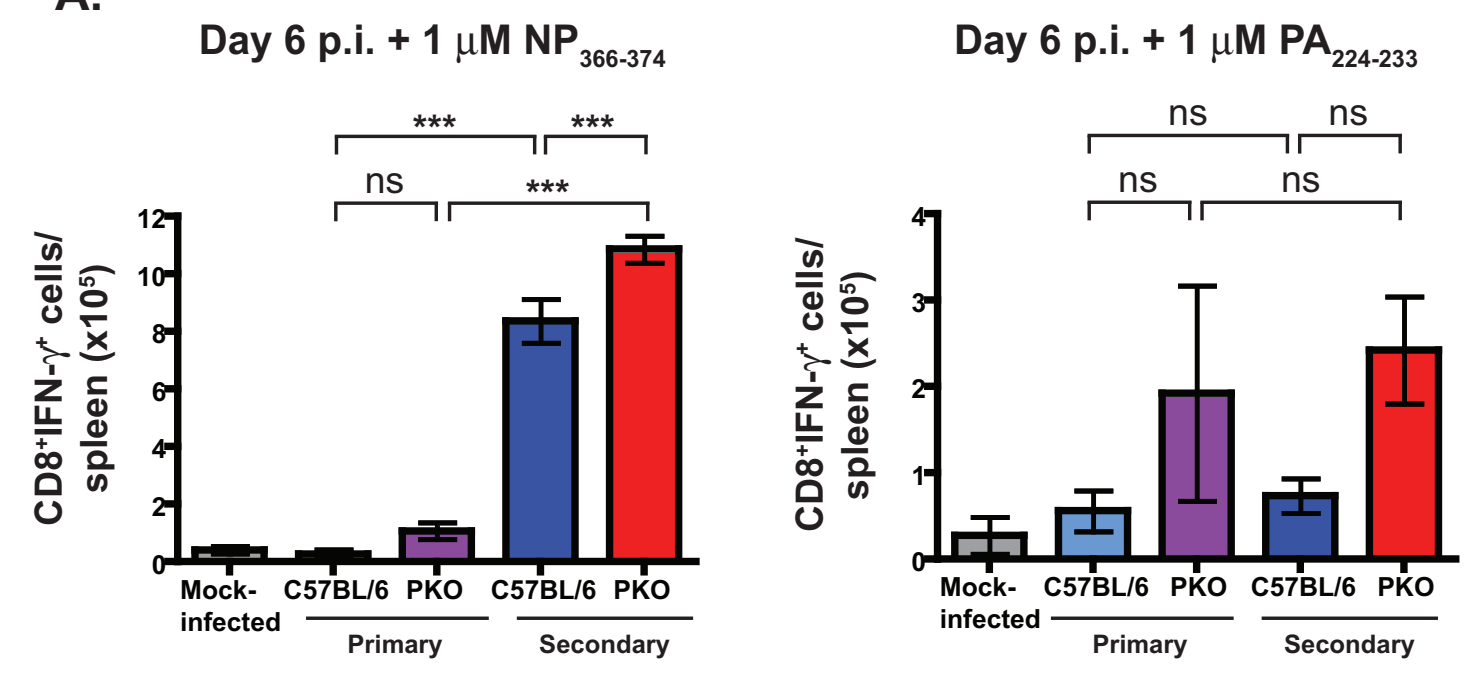

B.
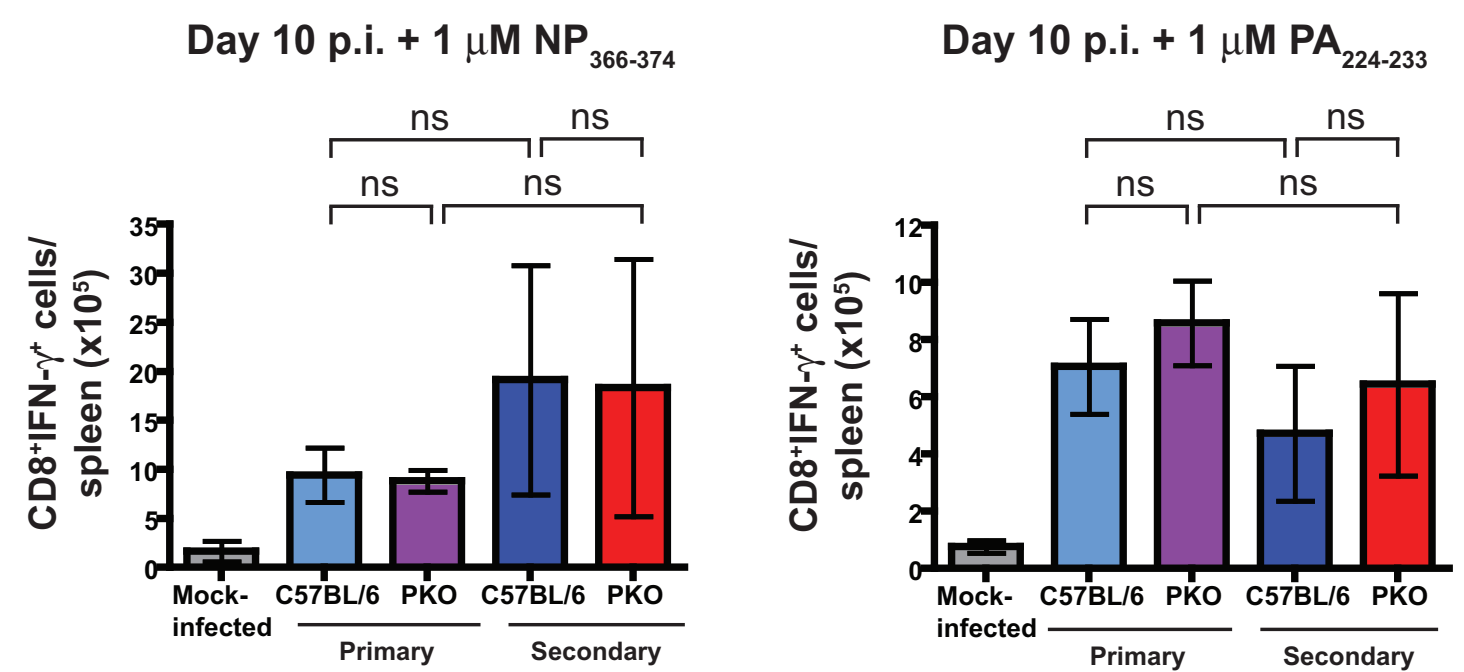

Figure 3.11 The number of antigen-specific $\mathrm{CD8}^{+} \mathrm{T}$ cells producing IFN- $\gamma$ in the spleen after a secondary influenza challenge.

C57BL/6 and PKO mice were infected i.n. with the HKx31 virus (Primary) or were infected i.p. with the PR8 virus and 30 days later infected i.n. with HKx31 virus (Secondary). As a negative control mice were mock-infected with PBS. At day 6 (A) and day 10 (B) after HKx31 virus infection the spleens were collected and processed. Cell suspensions were restimulated for $4 \mathrm{hr}$ with $1 \mu \mathrm{M} \mathrm{NP}{ }_{366-374}$ or $\mathrm{PA}_{224-233}$ peptide as indicated. Cells were labelled with anti-CD8 mAb, fixed and labelled intracellularly with anti-IFN- $\gamma \mathrm{mAb}$ and examined by flow cytometry. Lymphocytes were gated on the basis of their FSC vs. SSC properties. Each bar represents mean \pm SD or range of 2-3 mice per group. $* * *: p<0.001$, and ns: not significant by two-way ANOVA with a Tukey post test. 


\subsubsection{Expression of KLRG1 by influenza-specific $\mathrm{CD8}^{+} \mathrm{T}$ cells}

After prolonged or repetitive antigenic stimulation $\mathrm{CD}^{+} \mathrm{T}$ cells can progress into a state of exhaustion where they lose their effector function and fail to develop into memory cells (373). Therefore, it was of interest to determine whether the decreased $C D 8^{+} \mathrm{D}^{\mathrm{b}}-\mathrm{NP}_{366-374}{ }^{+}$ $\mathrm{T}$ cell response observed in the PKO mice during a secondary immune response was due to T cell exhaustion. KLRG1 has been found to be expressed on terminally differentiated $\mathrm{CD}^{+} \mathrm{T}$ cells but not on long lived memory cells (368-370). In addition, expression of KLRG1 has been associated with the presence of persistent antigen (371). This suggests a correlation between the expression of KLRG1 and T cell exhaustion. Therefore, the expression of KLRG1 on the influenza-specific $\mathrm{CD}^{+} \mathrm{T}$ cells in $\mathrm{PKO}$ and C57BL/6 mice was assessed to determine if the $\mathrm{CD}^{+} \mathrm{D}^{\mathrm{b}}-\mathrm{NP}_{366-374}{ }^{+} \mathrm{T}$ cells from the PKO mice had a greater expression of KLRG1. The percentage of $\mathrm{CD}^{+} \mathrm{D}^{\mathrm{b}}-\mathrm{NP}_{366-374}{ }^{+} \mathrm{T}$ cells that expressed KLRG1 was investigated in the blood 28 days after the secondary influenza challenge. After a viral infection the percentage of $\mathrm{CD} 8^{+} \mathrm{T}$ cells expressing KLRG1 increased in both the PKO and C57BL/6 mice (Figure 3.12 A). In addition, the PKO mice had a greater percentage of $\mathrm{CD}^{+} \mathrm{D}^{\mathrm{b}}-\mathrm{NP}_{366-374}{ }^{+} \mathrm{T}$ cells expressing KLRG1, compared to the C57BL/6 mice (29\% vs $19 \%$ ) (Figure 3.11 B). Thus this initial data would suggest that the reduced number of $\mathrm{CD}^{+} \mathrm{D}^{\mathrm{b}}-\mathrm{NP}_{366-374}{ }^{+} \mathrm{T}$ cells in $\mathrm{PKO}$ mice during a secondary infection could be due to T cell exhaustion.

Next it was of interest to determine the KLGR1 expression on $\mathrm{CD}^{+} \mathrm{D}^{\mathrm{b}}-\mathrm{PA}_{224-233}{ }^{+} \mathrm{T}$ cells as the percentage and number of these antigen-specific T cells did not differ between the two mouse strains (Figures 3.7, 3.8 and 3.11). The expression level of KLRG1 on NP 366 $374{ }^{+}$and $\mathrm{PA}_{224-233}{ }^{+}$responsive $\mathrm{T}$ cells was investigated 10 days after either a primary or secondary infection. There were no significant differences in the expression of KLRG1 on either the $\mathrm{NP}_{366-374}{ }^{+}$or $\mathrm{PA}_{224-233}{ }^{+}$responsive T cells from the MLN or spleen of both PKO and C57BL/6 mice 10 days after either a primary or secondary influenza infection (Figure 3.13). These data suggest that the inability of PKO mice to generate an increased $C D 8^{+} D^{b}$ $\mathrm{NP}_{366-374}{ }^{+} \mathrm{T}$ cell response compared to $\mathrm{C} 57 \mathrm{BL} / 6$ mice was not due to an increased expression of KLRG1 on these T cells. 


\section{Figure 3.12 Expression of KLRG1 on $\mathrm{CD8}^{+} \mathrm{D}^{\mathrm{b}}-\mathrm{NP}_{366-374}{ }^{+}$blood $\mathrm{T}$ cells after a secondary influenza infection.}

C57BL/6 and PKO mice were infected i.p. with the PR8 virus and 30 days later infected i.n. with HKx31 virus. As a negative control mice were mock-infected with PBS. Twenty eight days after HKx31infection, blood was collected from the tail vein and labelled with anti-CD8, $D^{\mathrm{b}}-\mathrm{NP}_{366-374}$ pentamer and anti-KLRG1. Dead $\left(\mathrm{PI}^{+}\right)$cells were excluded and lymphocytes were gated on the basis of their FSC vs. SSC properties. (A) Panels show representative dot plots of blood CD8 ${ }^{+}$ cells, $\mathrm{CD} 8^{+} \mathrm{KLRG} 1^{+}$cells are highlighted by an oval gate. (B) Panels show representative dot plots from 1 out of 2 mice, $\mathrm{CD} 8^{+} \mathrm{D}^{\mathrm{b}}-\mathrm{NP}_{366-374}{ }^{+}$cells (top panel) and $\mathrm{CD} 8^{+} \mathrm{D}^{\mathrm{b}}-\mathrm{NP}_{366-374}{ }^{+} \mathrm{KLRG}^{+}$(bottom panel) are highlighted by an oval gate. The specificity of anti-KLRG1 labelling was verified by comparing to a rat IgG1 isotype matched control antibody. 
A.
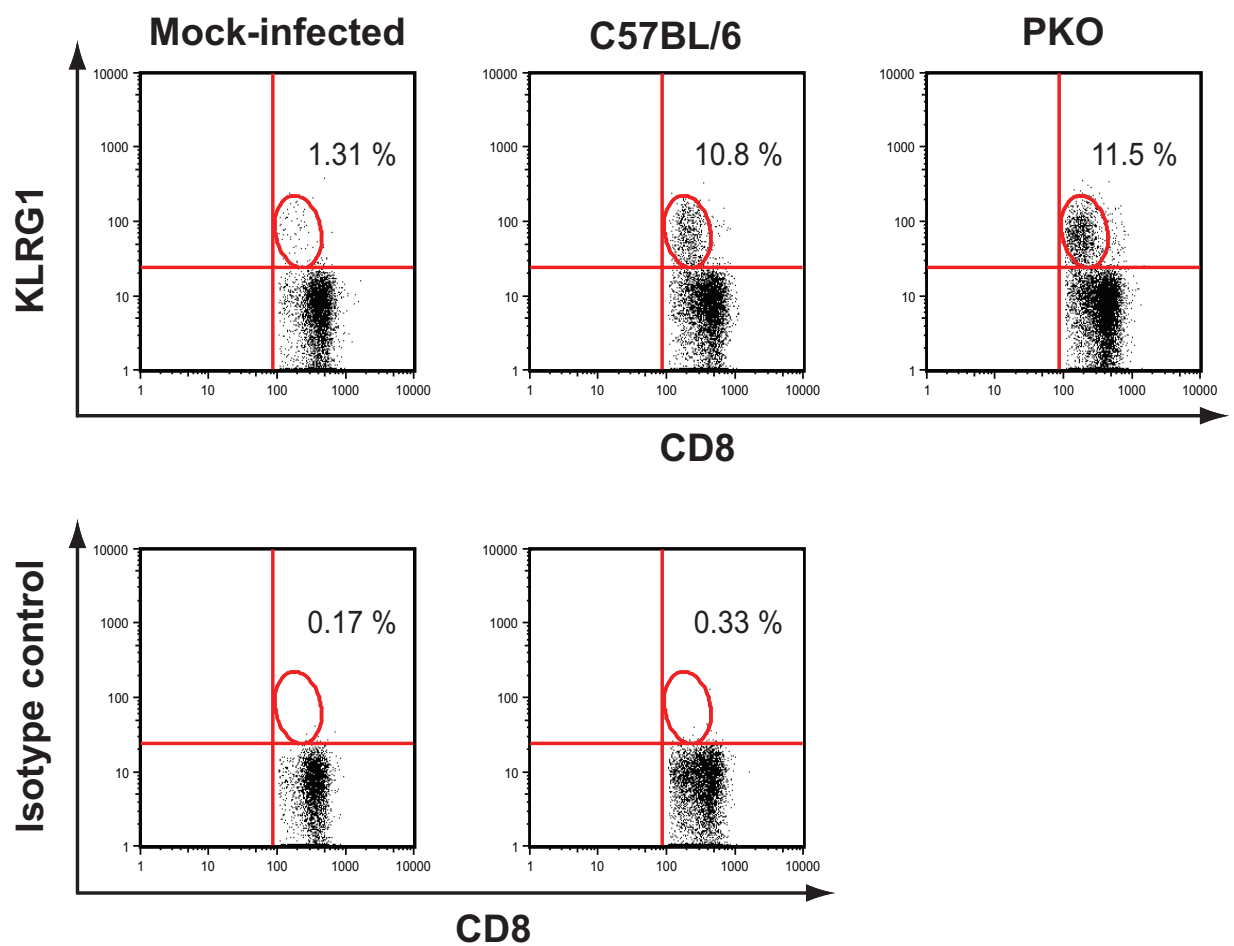

B.
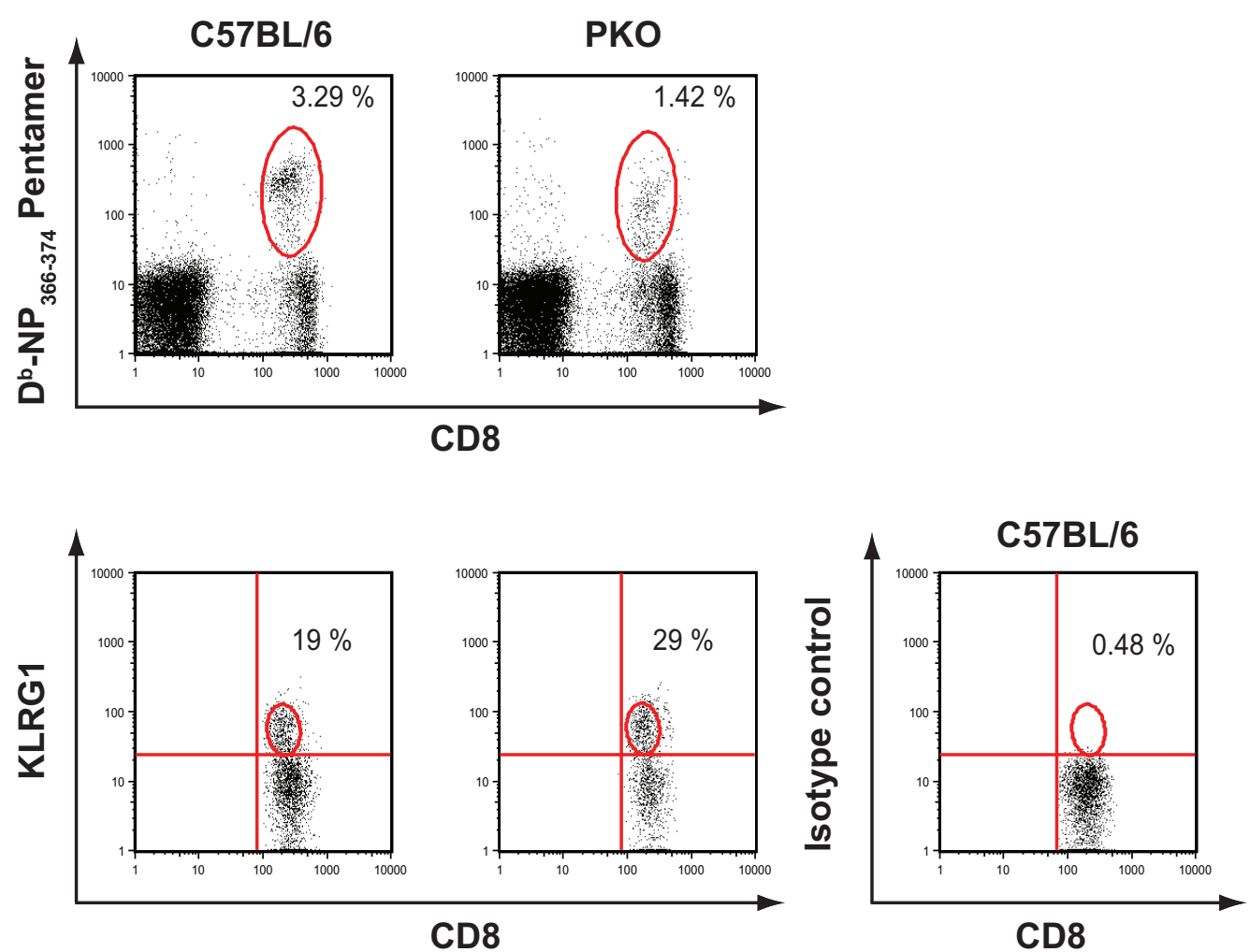


\section{Figure 3.13 The percentages of influenza-specific $\mathrm{CD8}^{+} \mathrm{T}$ cells expressing KLRG1 in} C57BL/6 and PKO mice are similar.

C57BL/6 and PKO mice were infected i.n. with the HKx31 virus (Primary) or were infected i.p. with the PR8 virus and 30 days later infected i.n. with HKx31 virus (Secondary). As a negative control mice were mock-infected with PBS. Ten days after HKx31 virus infection the MLN (A and B) and spleen (C and D) were collected. Tissues were processed and single cell suspensions made. MLN samples were digested in a solution of collagenase II and DNase I to obtain a single cell suspension. Cell suspensions were restimulated for $4 \mathrm{hr}$ with $1 \mu \mathrm{M} \mathrm{NP}{ }_{366-374}$ (A and C) or $\mathrm{PA}_{224-233}$ (B and D) peptide. Cells were labelled with anti-CD8 and anti-KLRG1 mAbs, fixed and labelled intracellularly with anti-IFN- $\gamma \mathrm{mAb}$ and examined by flow cytometry. Lymphocytes were gated on the basis of their FSC vs. SSC properties. Graphs show the percentage of $\mathrm{CD} 8^{+} \mathrm{IFN}-\gamma^{+}$cells that express KLRG1 on the cell surface in the MLN (A and B) and spleen (C and D) after restimulation with either $\mathrm{NP}_{366-374}\left(\mathrm{~A}\right.$ and $\mathrm{C}$ ) or $\mathrm{PA}_{224-233}$ peptide (B and D). Each data point represents an individual mouse and the line represents the mean. ns: not significant by a one-way ANOVA with a Tukey post test. 
A.

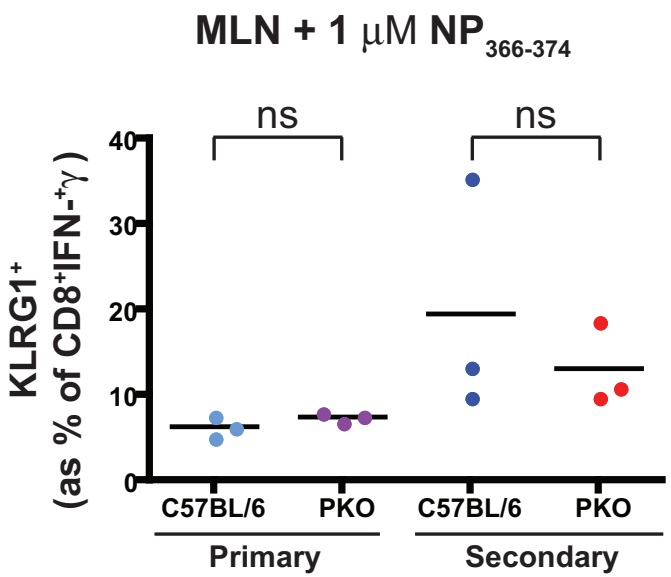

C.

Spleen $+1 \mu \mathrm{M} \mathrm{NP}_{366-374}$

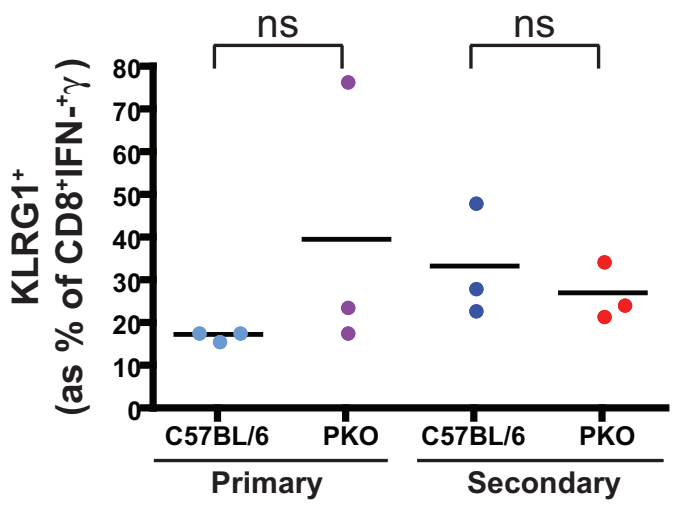

B.

$\mathrm{MLN}+1 \mu \mathrm{MPA}_{224-233}$

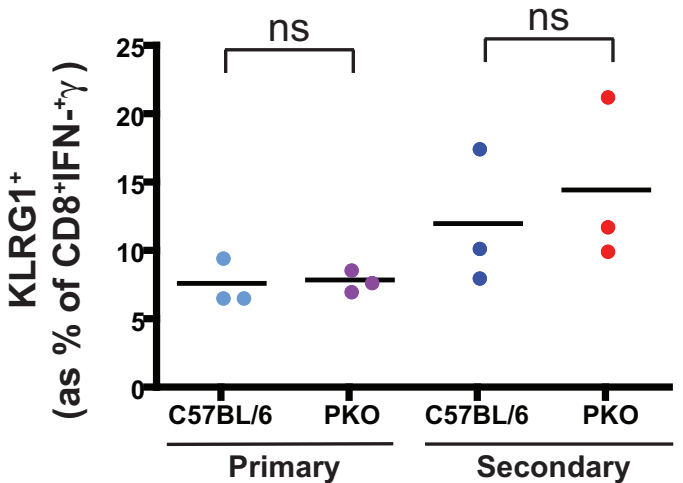

D.

Spleen + $1 \mu \mathrm{M} \mathrm{PA}_{224-233}$

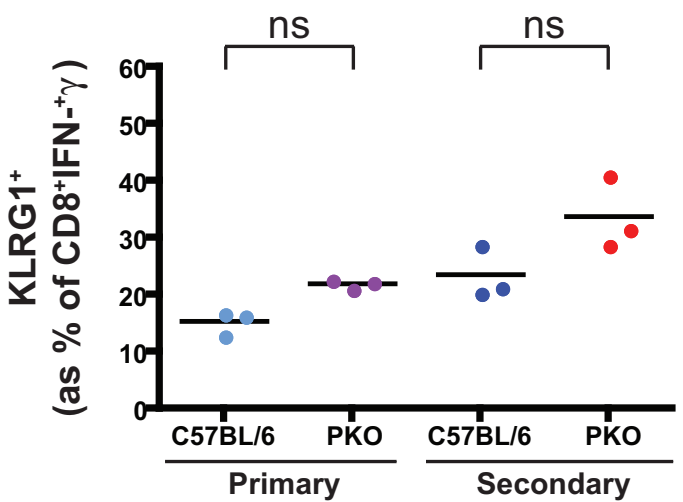




\subsection{Discussion}

The aim of the experiments described in this chapter was to characterise and compare the influenza-induced immune response in both C57BL/6 and PKO mice. It was hypothesised that PKO mice would have an increased number of antigen-specific $\mathrm{CD}^{+} \mathrm{T}$ cells compared to the C57BL/6 mice, due to a possible increased number of DC surviving in the PKO mice. The percentage and phenotype of antigen-specific $\mathrm{CD} 8^{+} \mathrm{T}$ cells were examined in multiple tissues throughout primary and secondary influenza infections. The frequency of influenza-specific $\mathrm{CD}^{+} \mathrm{T}$ cells during a primary influenza infection was similar in C57BL/6 and PKO mice. However, during a secondary influenza infection PKO mice had a markedly reduced frequency of $\mathrm{CD} 8^{+} \mathrm{D}^{\mathrm{b}}-\mathrm{NP}_{366-374}{ }^{+} \mathrm{T}$ cells compared to $\mathrm{C} 57 \mathrm{BL} / 6$ mice, while the frequency of $\mathrm{CD} 8{ }^{+} \mathrm{D}^{\mathrm{b}}-\mathrm{PA}_{224-233}{ }^{+} \mathrm{T}$ cells was similar between the two mouse strains. Additionally, the influenza-specific $\mathrm{CD}^{+} \mathrm{T}$ cells from both mouse strains similarly expressed the markers CD62L and KLRG1.

Similar to the findings of Liu et al and Topham et al, a slight delay in viral clearance in the PKO mice was found compared to C57BL/6 mice $(366,374)$. The delay in viral clearance correlated with a prolonged weight loss between 8 and 12 days after a primary infection in the PKO mice. This was in contrast with a more recent report where a similar rate of viral clearance was found in both the C57BL/6 and PKO mice (372). This difference from the earlier reports is not likely to be due to a variation in the detection system. Although, Price et al used an hemagglutination assay (372), this has been shown to have a similar sensitivity and specificity to the viral detection method used in this thesis (358). In contrast, the virus strain used in this study was the same used by Topham et al, whereas in the study by Price et al a different influenza virus strain of low virulence was used $(366,372)$. Consequently, subtle differences in the experimental system may account for the different results observed.

Studies using either an influenza vaccine (375) or influenza A virus (374) have shown that PKO mice generated an increased antigen-specific immune response compared to C57BL/6 mice. In addition, studies that used infection with LCMV or L. monocytogenes also reported that PKO mice had an increased antigen-specific $\mathrm{CD} 8^{+} \mathrm{T}$ cell response compared to WT mice $(338,339,376-378)$. These reports are in contrast to the results presented in this thesis, where 
no increase in percentage of antigen-specific $\mathrm{CD}^{+} \mathrm{T}$ cells was observed in $\mathrm{PKO}$ mice. Throughout a primary influenza infection the percentage of antigen-specific $\mathrm{CD} 8^{+} \mathrm{T}$ cells in the MLN, spleen and blood was similar between C57BL/6 and PKO mice. However, the majority of reports that demonstrated an enhanced immune response in the PKO mice used viral infections that required the presence of perforin for their clearance $(338,339,376-378)$. When PKO mice were infected with vesicular stomatitis virus (VSV), which does not require perforin for viral clearance, no enhanced immune response was seen in the PKO mice (379). Therefore it is possible that the enhanced $\mathrm{T}$ cell response observed by others in PKO mice is due to increased viral load and an inability to clear the virus. However, in the absence of a viral infection an enhanced antigen-specific $\mathrm{CD}^{+} \mathrm{T}$ cell response could still be induced in PKO mice compared to C57BL/6 mice (155). In that study, mice were exposed to limited antigen presented by adoptively transferred antigen loaded BMDC. Thus an increased viral load and inability to clear the virus does not completely explain the enhanced $\mathrm{T}$ cell responses observed by others in PKO mice. This will be further discussed in the general discussion chapter.

Throughout a secondary infection, C57BL/6 mice had a significant increase in the percentage of $\mathrm{CD}^{+} \mathrm{D}^{\mathrm{b}}-\mathrm{NP}_{366-374}{ }^{+} \mathrm{T}$ cells compared to a primary infection. This was in contrast to PKO mice where the percentage of $\mathrm{CD}^{+} \mathrm{D}^{\mathrm{b}}-\mathrm{NP}_{366-374}{ }^{+} \mathrm{T}$ cells did not significantly increase during the secondary immune response. Despite the fact that there was no significant increase in the percentage of $\mathrm{CD}^{+} \mathrm{D}^{\mathrm{b}}-\mathrm{NP}_{366-374}{ }^{+} \mathrm{T}$ cells in $\mathrm{PKO}$ mice, the response elicited during a secondary immune response did differ from the primary response. Firstly, during the secondary response $\mathrm{CD} 8^{+} \mathrm{T}$ cells were initially more activated, as a greater percentage was CD62 $\mathrm{L}^{\text {lo }}$. Secondly, the $\mathrm{CD} 8^{+} \mathrm{D}^{\mathrm{b}}-\mathrm{NP}_{366-374}{ }^{+} \mathrm{T}$ cells in the secondary response did not contract as greatly as those from the primary response. Thus, even though the $\mathrm{CD} 8^{+} \mathrm{D}^{\mathrm{b}}-\mathrm{NP}_{366-374}{ }^{+} \mathrm{T}$ cells in the PKO mice did not expand significantly more in the secondary response in comparison to the primary response, they still show characteristic features of memory cells.

During secondary influenza infection the percentage of $\mathrm{CD}^{+} \mathrm{D}^{\mathrm{b}}-\mathrm{NP}_{366-374}{ }^{+} \mathrm{T}$ cells in PKO mice did not increase to the extent seen in C57BL/6 mice. This could have been due to an increased expansion of $\mathrm{CD}^{+} \mathrm{T}$ cells specific for the $\mathrm{D}^{\mathrm{b}}-\mathrm{PA}_{224-233}$ epitope. It has previously been shown that during a chronic viral infection the immunodominance hierarchy was altered, 
with the subdominant epitopes becoming more dominant (380). The increased antigen presentation by DC in the PKO mice during a secondary influenza infection (156) could mimic a situation similar to chronic viral infection. However, previously published data have shown that the immunodominance hierarchy of the $\mathrm{D}^{\mathrm{b}}-\mathrm{NP}_{366-374}$ and $\mathrm{D}^{\mathrm{b}}-\mathrm{PA}_{224-233}$ epitopes was intact in PKO mice during primary and secondary influenza infections after an i.p. influenza administration $(381,382)$. This route of administration was in contrast to the i.n. infection route used in this study. Thus, it was important to examine the immunodominance hierarchy in this study where a viral infection was established, as the presence of viral antigens may have affected the response in PKO mice. Similar to these earlier studies $(381,382)$, results in this thesis discovered no difference in the percentage of $\mathrm{CD} 8^{+} \mathrm{D}^{\mathrm{b}}-\mathrm{PA}_{224-233}{ }^{+} \mathrm{T}$ cells between the two mouse strains. Taken together, these data indicate that a difference in the dominance hierarchy of the epitopes does not account for the lack of expansion in the $\mathrm{CD} 8^{+} \mathrm{D}^{\mathrm{b}}-\mathrm{NP}_{366-374}{ }^{+}$ $\mathrm{T}$ cells observed in PKO mice.

The frequency of antigen-specific $\mathrm{T}$ cells was assessed by the production of antigen-specific IFN- $\gamma$ or by using MHC I pentamers. These two techniques have been shown to produce comparable results $(177,356,372)$. In experiments described in this thesis the IFN- $\gamma$ assay and MHC I labelling resulted in equivalent percentages of antigen-specific $\mathrm{CD} 8^{+} \mathrm{T}$ cells during a primary response, however, during a secondary response the number of IFN- $\gamma$ producing $\mathrm{CD}^{+} \mathrm{T}$ cells and percentage of MHC I pentamer positive cells did not completely correlate. Using the MHC I pentamer it was found that the percentage of $\mathrm{CD}^{+} \mathrm{D}^{\mathrm{b}}-\mathrm{NP}_{366-374}{ }^{+} \mathrm{T}$ cells was greater in C57BL/6 mice than PKO mice during a secondary response, whereas a significant difference in the number of $\mathrm{CD}^{+} \mathrm{D}^{\mathrm{b}}-\mathrm{NP}_{366-374}{ }^{+} \mathrm{T}$ cells producing IFN- $\gamma$ was detected only at an early time point. One reason for the difference in these two experimental results could be due to the different tissues examined. The MHC I pentamer data was obtained from the peripheral blood whereas the IFN- $\gamma$ data was generated from lymphoid tissue. In contrast, other studies that showed the two techniques to be comparable directly assessed the techniques in the same tissue $(177,356,372)$. Additional explanations for the lack of correlation in this experimental system could be that the $\mathrm{CD} 8^{+} \mathrm{D}^{\mathrm{b}}-\mathrm{NP}_{366-374}{ }^{+} \mathrm{T}$ cells in $\mathrm{C} 57 \mathrm{BL} / 6$ mice are not all fully functional and not producing IFN- $\gamma$ or that the PKO mice produced more IFN- $\gamma$ to compensate for the lack of perforin. However, if after the data has been repeated a similar 
result is observed, this would suggest that the frequency of antigen-specific $\mathrm{CD} 8^{+} \mathrm{T}$ cells differs between peripheral and lymphoid tissues, which could arise from altered migration. Further investigations are required to determine why the results from the IFN- $\gamma$ assay did not correlate with the data obtained using the MHC I pentamer.

Situations of increased DC survival have resulted in increased T cell responses $(144,158,160$, $161)$ and improved tumour protection (160). In addition, enhanced immune responses have been observed in PKO mice during a range of infections (338-340, 377). This enhanced immune response has been proposed to be due to the increased number of DC presenting antigen, as they are not eliminated by the CTL due to the lack of perforin (155). Thus it was hypothesised that during an influenza infection PKO mice would have an increased $\mathrm{T}$ cell response and that this would correlate with increased DC survival. However, an enhanced T cell response in PKO mice was not observed in experiments described in this thesis. In fact, during a secondary influenza infection PKO mice showed a markedly reduced $C D 8^{+} \mathrm{D}^{\mathrm{b}}-\mathrm{NP}_{366-}$ $374^{+} \mathrm{T}$ cell response compared to C57BL/6 mice. These data would suggest that if there is a difference in DC survival in PKO mice during an influenza infection it is not reflected in the influenza specific $\mathrm{T}$ cell response.

The next step in this study was to investigate the number of DC in both C57BL/6 and PKO mice, however, during the course of this study a report by Belz et al was published that also investigated whether CTL regulate antigen presentation in a perforin-dependent manner during an influenza infection (156). The experimental approach used in their study differed from the method used in this thesis. Belz et al examined the number of cells presenting $\mathrm{NP}_{366-}$ 374 and $\mathrm{PA}_{224-233}$ epitopes in the MLN by using specific T cell hybridomas expressing LacZ. Cells taken from MLN at differing time points during the course of an influenza infection were cultured with specific $\mathrm{LacZ}^{+} \mathrm{T}$ cell hybridomas, and the number of cells in MLN presenting influenza epitopes was determined by calculating the number of $\mathrm{LacZ}^{+}$hybrids. Using this approach it was demonstrated that during a secondary influenza infection there was a greater number of cells presenting influenza-specific epitopes in the MLN of PKO mice compared to C57BL/6 mice (156), suggesting that memory $\mathrm{CD} 8^{+} \mathrm{T}$ cells eliminate $\mathrm{DC}$ in a perforin-dependent manner. This result is in complete contrast to the findings presented in this 
thesis, where the absence of an enhanced T cell response in PKO mice implies that CTL do not eliminate DC in a perforin-dependent manner during an influenza infection. As the current study and the report by Belz et al (156) used alternative experimental approaches to address the same question it makes it difficult to directly compare the two studies as different parameters were measured. However, as the viral load was not examined in the study by Belz et al (156), it may have been increased in PKO mice, which could account for the opposing results between the two studies. In the steady state there is continual migration of lung DC to the draining LN and following a lung infection this migration of lung DC rapidly increases (383). It is feasible that prolonged presence of viral antigens could further increase the migration of lung DC to the draining LN, and account for the observed increase in cells presenting influenza antigens in the MLN of PKO mice (156). Since a delayed viral clearance in PKO mice has been observed during a primary influenza infection $(366,374)$, PKO mice might also have an increased viral load during a secondary influenza infection. Belz et al (156) did use the same viral strains as this thesis (PR8 and HKx31), and results described in Chapter 3 suggest that based on the similar weight loss between C57BL/6 and PKO mice that the viral loads are comparable during a secondary influenza infection. However, the viral stocks of PR 8 and HKx31 used by Belz et al may have been more virulent than those used in this current study, which could have impacted on the viral load in PKO mice. Therefore, it would have been valuable for Belz et al (156) to evaluate the viral load in the two mouse strains to confirm that viral load was not a factor in the observed findings. Nevertheless, it is possible that PKO mice do have an increased number of antigen presenting DC in the MLN, but this does not correlate with an increased $\mathrm{T}$ cell response, rather the prolonged antigen presentation is detrimental to an effective T cell response. Further investigations, such as assessing both the survival of DC presenting influenza antigens and the influenza-specific $\mathrm{T}$ cell response in the same experimental setting are necessary to ascertain whether DC survival and the magnitude of the T cell response correlate during an influenza infection.

In situations of chronic infection and antigen persistence, antigen-specific $\mathrm{CD} 8^{+} \mathrm{T}$ cells have been found to become exhausted and die (373). The prolonged antigen presentation observed in PKO mice during a secondary influenza infection might result in the influenza-specific $\mathrm{T}$ cells becoming exhausted and dying. KLRG1 has been reported to be a marker of terminal 
effector cells and of cell exhaustion (368-370). When expression of KLRG1 on the influenzaspecific $\mathrm{CD}^{+} \mathrm{T}$ cells in $\mathrm{C} 57 \mathrm{BL} / 6$ and $\mathrm{PKO}$ mice was investigated, a trend towards increased expression during the secondary response compared to the primary response was observed. This was consistent with other published data (384), which investigated the expression of KLRG1 on $\mathrm{CD}^{+} \mathrm{T}$ cells during immunisation regimens with $L$. monocytogenes and VSV. However, studies in this thesis showed there was no statistical difference in the expression of KLRG1 between the PKO and C57BL/6 mice. This lack of statistical difference between the C57BL/6 and PKO mice could have arisen from not analysing the optimal time point.

However, work by Masopust et al investigated the expression of KLRG1 on antigen-specific $\mathrm{CD}^{+} \mathrm{T}$ cells during a secondary infection and found that KLRG1 expression was maintained for the duration of the infection (384), suggesting that at the time points analysed in this thesis the influenza-specific $\mathrm{CD} 8^{+} \mathrm{T}$ cells should still be expressing KLRG1. Therefore this suggests the lack of difference in expression of KLRG1 between the C57BL/6 and PKO mice was a real effect.

The use of the KLRG1 marker may not be enough to definitively identify terminally differentiated and exhausted $\mathrm{CD} 8^{+} \mathrm{T}$ cells. During an extensive analysis of markers expressed during $\mathrm{T}$ cell exhaustion, KLRG1 was found to be expressed at low levels on $\mathrm{CD} 8^{+} \mathrm{T}$ cells from mice infected with the chronic LCMV strain compared to those infected with the acute LCMV strain (385). This was in contrast to studies that found KLRG1 associated with repetitive antigen stimulation, cellular senescence, and terminal differentiation $(369,370,384$, 386). Therefore, expression of KLRG1 by itself may not be sufficient to establish whether or not a $\mathrm{T}$ cell has an exhausted phenotype. PD-1, along with a number of other inhibitory cell markers, is also associated with T cell exhaustion $(385,387)$. Through kinetic analysis of a large array of markers it appears that $\mathrm{CD}^{+} \mathrm{T}$ cell exhaustion is a gradual process during chronic viral infection and that there are stages in the differentiation and development of exhausted $\mathrm{CD} 8^{+} \mathrm{T}$ cells (385). Thus further investigations are required to determine whether the $\mathrm{CD} 8^{+} \mathrm{T}$ cells in the PKO mice are in a state of exhaustion.

The lung epithelium could play an inhibitory role leading to the lack of expansion of the CD8 ${ }^{+}$ $\mathrm{T}$ cells in the PKO mice during a secondary influenza infection. Airway epithelial cells produce an array of regulatory mediators including a number of chemokines and cytokines 
(388) that could have an inhibitory effect on both $\mathrm{DC}$ and $\mathrm{CD} 8^{+} \mathrm{T}$ cells after an influenza infection. As PKO mice are unable to kill target cells via the perforin/granzyme pathway, more infected epithelial cells would be present, potentially leading to an increased level of inhibitory factors. Thus in PKO mice the expansion of the $\mathrm{CD} 8^{+} \mathrm{D}^{\mathrm{b}}-\mathrm{NP}_{366-374}{ }^{+} \mathrm{T}$ cells could be restricted by inhibitory mediators released from epithelial cells. Further investigation into the levels of regulatory chemokines and cytokines secreted by lung epithelial cells would be required to ascertain if this is a potential mechanism and determine why an effect is seen only during a secondary and not a primary immune response.

The PKO and C57BL/6 mice were able to generate an effective primary influenza-specific immune response but the $\mathrm{CD}^{+} \mathrm{T}$ cell response specific for the dominant epitope during a secondary response was dramatically reduced in PKO mice. These data suggest that perforin may be involved in some aspect of $\mathrm{CD}^{+} \mathrm{T}$ cell memory response against influenza virus. Over the last few years many factors have been discovered to be significant in the generation of long-lived memory $\mathrm{CD} 8^{+} \mathrm{T}$ cells, such as the transcription factors $\mathrm{T}$ bet, Eomes and BLIMP-1 along with the cytokines IL-7 and IL-15 $(389,390)$. It is likely that over the coming years more factors will be discovered that are important for the generation of an effective $\mathrm{CD}^{+} \mathrm{T}$ cell response. Thus perhaps perforin is also important for the maintenance of $\mathrm{CD} 8^{+} \mathrm{T}$ cell memory. However, it has been demonstrated that PKO mice can clear a secondary influenza viral infection at a similar rate to the C57BL/6 mice (372), although this was shown using an influenza strain of low virulence, but it would suggest that under certain conditions memory $\mathrm{CD}^{+} \mathrm{T}$ cells that lack perforin are present and able to clear the viral infection. Further investigation is needed to determine how perforin would confer this improved memory $\mathrm{CD}^{+} \mathrm{T}$ cell response.

\subsubsection{Summary}

C57BL/6 and PKO mice have a similar disease progression during both a primary and secondary influenza virus infection. Throughout a primary influenza infection there was no evidence indicating that the percentages of $\mathrm{CD}^{+} \mathrm{D}^{\mathrm{b}}-\mathrm{NP}_{366-374}{ }^{+}$or $\mathrm{CD}^{+} \mathrm{D}^{\mathrm{b}}-\mathrm{PA}_{224-233}{ }^{+} \mathrm{T}$ cells was different between the two mouse strains. This was in contrast to the secondary infection 
where C57BL/6 mice had a statistically significant 3-fold increase in the percentage of $\mathrm{CD} 8^{+} \mathrm{D}^{\mathrm{b}}-\mathrm{NP}_{366-374^{+}} \mathrm{T}$ cells compared to PKO mice. This lack of secondary $\mathrm{T}$ cell expansion in PKO mice did not appear to result from exhaustion as the expression of KLRG1 on the antigen-specific $\mathrm{CD} 8^{+} \mathrm{T}$ cells was similar in PKO mice and C57BL/6 mice. However, before $\mathrm{T}$ cell exhaustion can be ruled out completely other markers of $\mathrm{T}$ cell exhaustion need to be investigated. Furthermore, the antigen-specific $\mathrm{CD} 8^{+} \mathrm{T}$ cells in the two mouse strains had a similar expression of CD62L during both a primary and secondary infection, indicating a similar activation status. Overall, these data indicate that in this influenza model, PKO mice do not generate an enhanced influenza specific T cell response compared to C57BL/6 mice. This would suggest that the increased antigen presentation in PKO mice reported by others (156) does not correlate with an enhanced influenza-specific $\mathrm{CD} 8^{+} \mathrm{T}$ cell response. 


\section{Chapter 4:}

\section{Differential survival of splenic DC subsets after treatment with $\alpha$-GalCer}




\subsection{Introduction}

DC survival is important due to the central role DC play in the generation of effective immune responses. As discussed in section 1.4, investigations have demonstrated that CTL eliminate adoptively transferred antigen loaded BMDC (155) and endogenous DC presenting influenza antigen during a secondary influenza immune response (156) in a perforin-dependent manner. However, CTL are not the only cells that have the ability to eliminate target cells. A number of in vitro studies have demonstrated that both mouse and human NKT cells can kill tumour target cells $(313-315,318,323)$, while human NKT cells can kill DC (150-152, 310, 313). In vitro investigations into the mechanism of DC killing revealed that NKT cells can kill DC in a perforin-dependent manner (310). Consequently, NKT cells might also regulate DC survival in vivo in a perforin-dependent mechanism similar to CTL.

To assess whether NKT cells regulated DC survival in a perforin-dependent manner, an in vivo model was established that consisted of i.v. treatment of C57BL/6 and PKO mice with the glycolipid, $\alpha$-GalCer, which is a potent activator of NKT cells. This enabled the evaluation of whether NKT cells eliminate DC in an in vivo setting and to determine whether the mechanism of elimination was dependent on perforin. Furthermore, $\alpha$-GalCer administered in conjunction with the model protein antigen ovalbumin (ova) activates stronger ova-specific $\mathrm{CD}^{+}$and $\mathrm{CD}^{+} \mathrm{T}$ cell responses compared to ova protein alone $(319,320,325,326)$. Therefore, this $\alpha$-GalCer/ova protein model also allowed us to investigate whether specific CTL can mediate DC elimination during a different immune response than those previously studied.

Activation of NKT cells through the administration of $\alpha$-GalCer also induces B cell activation, proliferation, antibody secretion and expansion of the B cell memory pool (327, 330, 332, 335). Furthermore, treatment with $\alpha$-GalCer together with either ova protein or particulate antigen has led to the production of IgM and class switched specific antibodies including ova-specific $\operatorname{IgE}(329,331-333)$. Taken together, treatment with the NKT cell ligand, $\alpha$-GalCer and ova protein elicits both antigen-specific $\mathrm{T}$ and $\mathrm{B}$ cell responses. The elimination of DC by NKT cells could have an impact on both these immune responses as DC are critical to NKT cell activation $(391,392)$. 


\subsection{Aims}

The experiments described in this chapter were designed to evaluate whether DC survival was regulated in a perforin-dependent manner after treatment of $\alpha$-GalCer and ova protein.

The specific aims were:

- To determine whether the number of splenic DC decreased during treatment with $\alpha-$ GalCer and ova protein, and whether this decrease required perforin.

- To determine whether PKO mice generated an enhanced ova-specific $\mathrm{CD} 8^{+} \mathrm{T}$ cell response after treatment with $\alpha$-GalCer and ova protein compared to C57BL/6 mice.

- To determine whether DC survival is regulated in a perforin-dependent manner during an ova-specific secondary $\mathrm{T}$ cell response.

\subsection{Results}

\subsection{1 $\mathrm{CD8}^{+} \mathrm{DC}$ are depleted by treatment with $\alpha$-GalCer and ova protein}

To determine whether DC were eliminated after i.v. administration of $\alpha$-GalCer and ova protein, the number of DC in the spleens of C57BL/6 mice was examined $48 \mathrm{hr}$ after treatment. DC were identified on the basis of cell surface expression of CD11c and MHC II, and quantified by flow cytometry (Figure 4.1). No significant difference in the total number of DC was observed when mice were treated with $\alpha$-GalCer with or without ova protein compared to control groups (Figure 4.1).

DC are a heterogeneous cell population and have been divided into subsets on the basis of the expression of a number of cell surface markers. Experimental evidence suggests that these DC subsets have different roles in immune responses (46) and express different TLR, 
and thus are sensitive to different stimuli (49). The expression of the cell surface marker CD8 was used to differentiate between the $\mathrm{CD} 8^{+}$and $\mathrm{CD} 8^{-} \mathrm{DC}$ subsets. It was observed that there was a decrease in the number of $\mathrm{CD}^{+} \mathrm{DC}$ after treatment with $\alpha$-GalCer, and that this occurred in the absence or presence of ova protein (Figure $4.2 \mathrm{~A}$ and $\mathrm{B}$ ). The number of $\mathrm{CD}^{-} \mathrm{DC}$ did not change, though both $\mathrm{CD} 8^{+}$and $\mathrm{CD} 8^{-} \mathrm{DC}$ subsets showed an increased cell surface expression of CD86 (Figure 4.2). This indicated that both DC subsets were activated upon treatment with $\alpha$-GalCer and ova protein, and is consistent with previously published data (187). These results indicated that different DC subsets responded differently to treatment with $\alpha$-GalCer; the $\mathrm{CD} 8^{+} \mathrm{DC}$ subset declined, while the CD8 ${ }^{-}$DC subset did not change. The presence of ova protein along with $\alpha$-GalCer did not alter the response compared to $\alpha$-GalCer only, thus ova-specific T cells were not involved in the loss of $\mathrm{CD} 8^{+} \mathrm{DC}$ in this model. 
A.

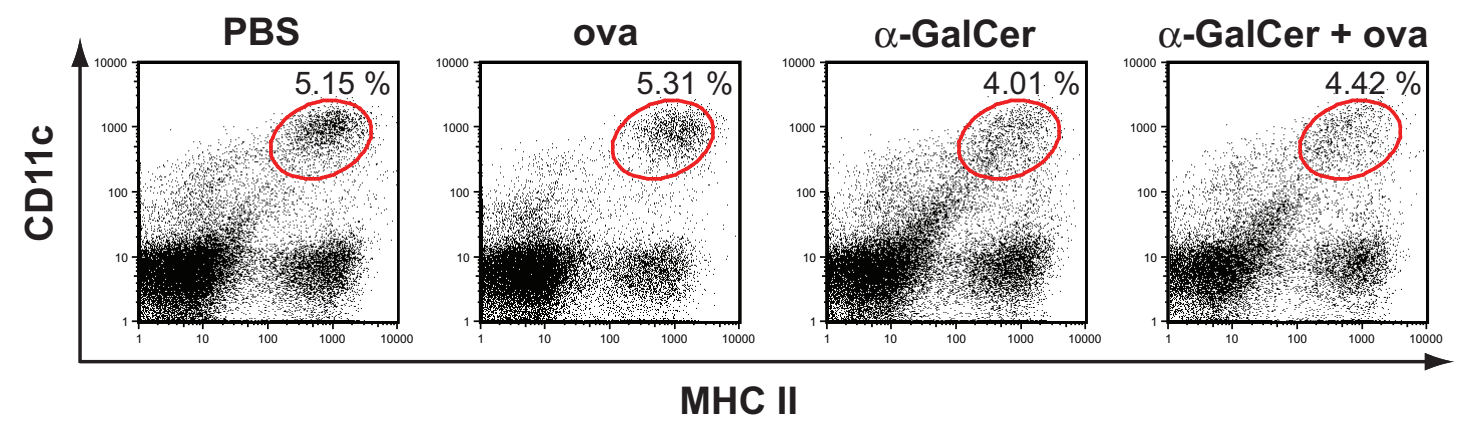

B.

CD11c ${ }^{+} \mathrm{MHC} \mathrm{II}^{+}$cells

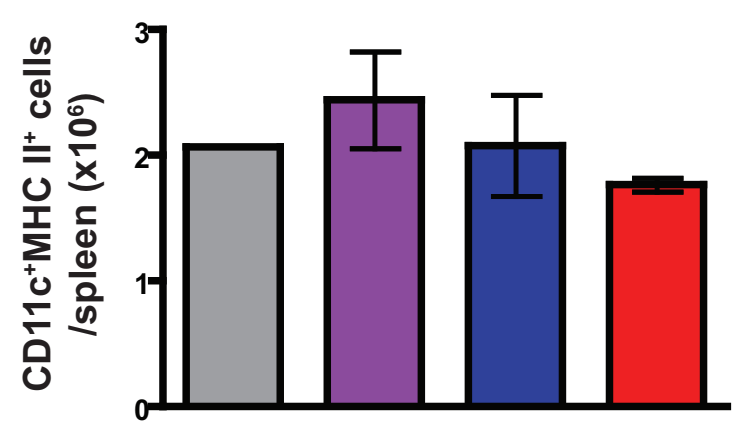

Key: $\square$ PBS

Lova

$\square \alpha-$ GalCer

$\square \alpha-$ GalCer + ova

Figure 4.1 Treatment with $\alpha$-GalCer and ova protein does not affect the total number of splenic DC.

C57BL/6 mice were injected i.v. with $\alpha-G a l C e r$ and/or ova protein as indicated. Spleens were harvested $48 \mathrm{hr}$ later and the number of DC present in the spleen was analysed by flow cytometry. Dead $\left(\mathrm{PI}^{+}\right)$and B220 cells were excluded. Lymphocytes were gated on the basis of their FSC vs. SSC properties. (A) Representative dot plots of spleen samples; the $\mathrm{CD} 11 \mathrm{c}^{+} \mathrm{MHC} \mathrm{II}^{+}$population is highlighted by an oval, the percentage of these cells in the total gated population is indicated. (B) Total number of DC in the spleen $48 \mathrm{hr}$ after the indicated treatment. Bars represent mean \pm range of 2 mice per group, except the PBS control group where only one mouse was examined. This experiment was repeated with similar results. 
Figure 4.2 Treatment with $\alpha$-GalCer induces a decrease in the number of splenic $\mathrm{CD8}^{+} \mathrm{DC}$.

C57BL/6 mice were injected i.v. with $\alpha$-GalCer and/or ova protein as indicated. Spleens were harvested 24 or $48 \mathrm{hr}$ later and analysed as described in Figure 4.1. (A) Dot plots of representative spleen samples; the CD8 ${ }^{-} \mathrm{DC}$ are indicated by a rectangle gate and the $\mathrm{CD} 8^{+} \mathrm{DC}$ are indicated by an oval, the percentage of these cells in the total gated population is indicated. (B) Total number of CD8 ${ }^{-}$DC (left panel) and CD8 ${ }^{+}$DC (right panel) present in the spleen $48 \mathrm{hr}$ after the indicated treatments. (C) The expression of CD86 on the splenic CD8 ${ }^{-}$DC (left panel) and CD8 ${ }^{+}$DC (right panel) $24 \mathrm{hr}$ after the indicated treatments. Bars represent mean \pm SD of 3-4 mice per group. $* * *: p$ $<0.001, *: p<0.05$, and ns: not significant by one-way ANOVA with a Tukey post test. These experiments were repeated with similar results. 
A.
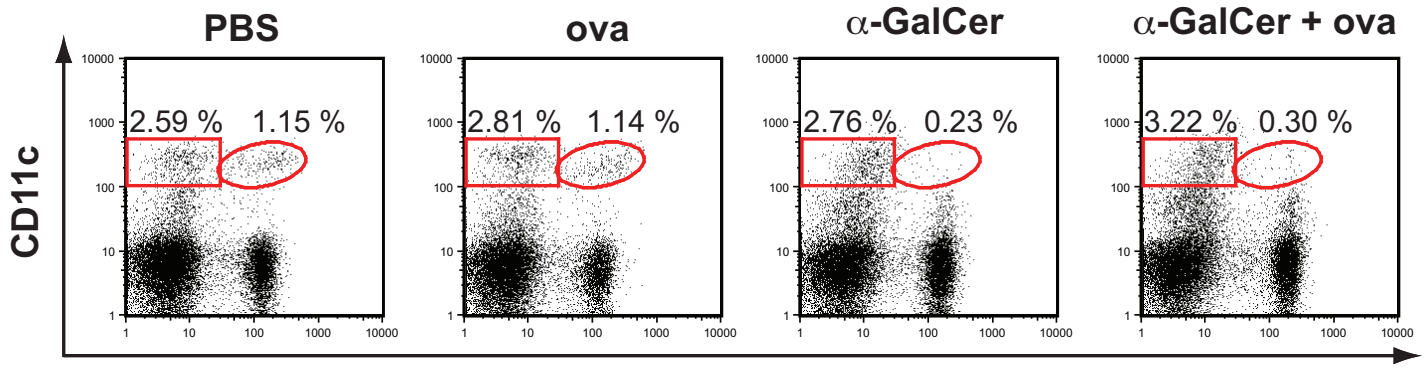

CD8

B.

CD8- DC

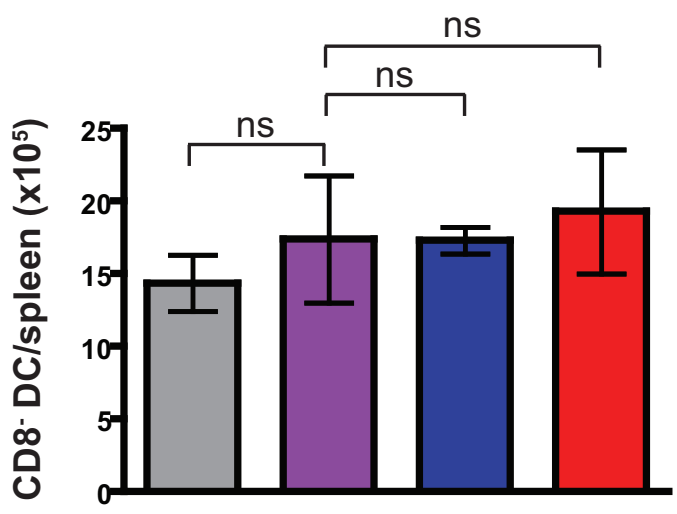

Key: $\square$ PBS $\square$ ova

C.

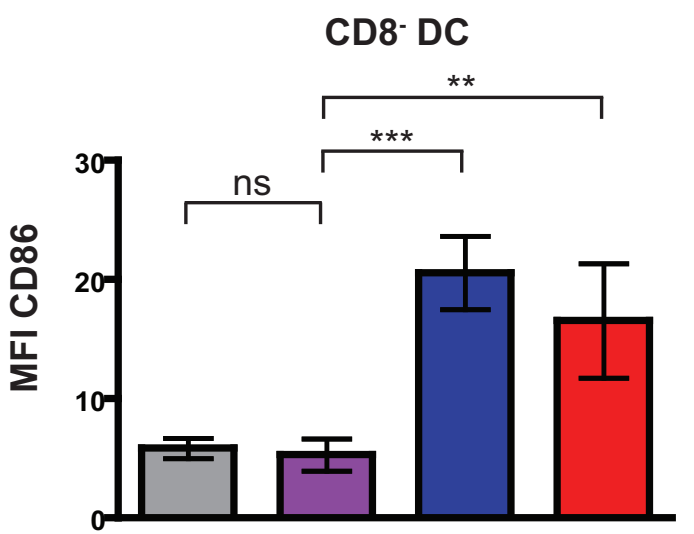

CD8+ DC

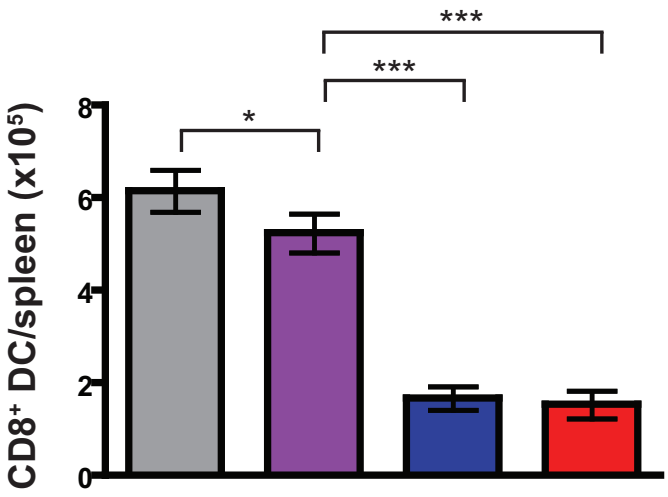

$\alpha-$ GalCer

$\alpha-$ GalCer + ova

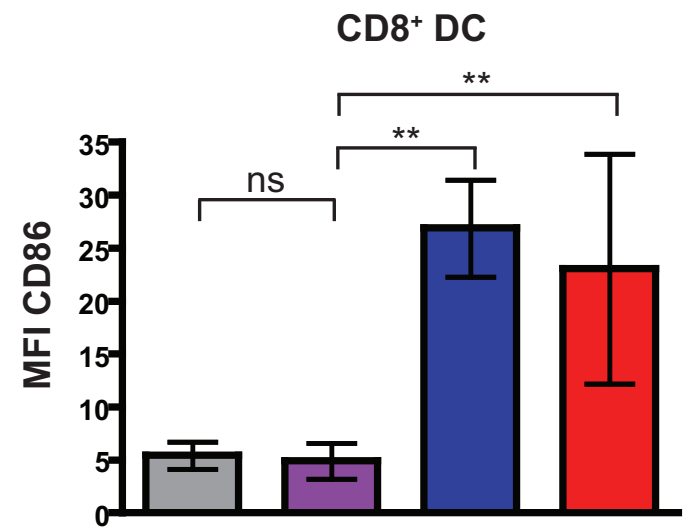




\subsubsection{The decrease in the splenic $\mathrm{CD8}^{+} \mathrm{DC}$ subset is not due to perforin- dependent killing}

The results in Figure 4.2 indicated that loss of $\mathrm{CD} 8^{+} \mathrm{DC}$ after treatment with $\alpha$-GalCer and ova protein was not due to the presence of ova-specific T cells. The loss of $\mathrm{CD} 8^{+} \mathrm{DC}$ was observed after treatment with the NKT cell ligand, thus it was considered likely that the NKT cells were mediating the loss of $\mathrm{CD} 8^{+} \mathrm{DC}$. NKT cells have cytotoxic ability and are reported to kill DC presenting $\alpha$-GalCer in vitro (150). Blocking perforin-mediated killing has been reported to significantly reduced the ability of NKT cells to kill tumour cell lines (312-315) and human DC in vitro (310). Therefore it was of interest to determine whether the observed decrease in $\mathrm{CD}^{+} \mathrm{DC}$ required the expression of perforin, especially as CTL have been shown to eliminate DC in vivo via a perforin-dependent mechanism $(155,156)$. To investigate this, PKO mice were treated with $\alpha$-GalCer i.v., and the number of splenic CD $8^{+} \mathrm{DC}$ was determined $24 \mathrm{hr}$ later. As shown in Figure 4.3, C57BL/6 and PKO mice treated with $\alpha$ GalCer had similar decreases in the number of $\mathrm{CD} 8^{+} \mathrm{DC}$, while the number of $\mathrm{CD} 8^{-} \mathrm{DC}$ was not affected in either strain of mice. Additionally, both C57BL/6 and PKO mice responded to the treatment of $\alpha$-GalCer with similar increases in the expression of CD86 on both DC subsets (Figure 4.3). These data indicated that the loss of CD8 ${ }^{+} \mathrm{DC}$ subset after $\alpha$-GalCer treatment was not due to perforin-dependent killing.

Human in vitro experiments have demonstrated that NKT cells can kill DC in a perforindependent manner (310), however, it has not been demonstrated if this occurs in vivo. Mouse NKT cells have been reported to kill $\alpha$-GalCer loaded splenocytes in an antigen-specific manner, but the mechanism of this target cell lysis was not established (360). Therefore, it was next asked if mouse NKT cells could kill target cells in vivo in a perforin-dependent manner. To assess this splenocytes were loaded with or without $\alpha$-GalCer, and labelled with the fluorescent dyes CFSE or CTO, respectively, to track the two splenocyte populations. These labelled splenocytes were injected i.v. into C57BL/6, PKO or CD1 deficient (CD1 KO) mice and MesLN were recovered $24 \mathrm{hr}$ later to quantify the percentage of the remaining labelled splenocyte populations. CD1 KO mice, which lack NKT cells, were used as a control to establish a baseline for NKT cell specific killing, as any target cell loss in these hosts could 
only be explained by non-specific cell loss. The percentage of antigen-specific killing in C57BL/6 and PKO mice was calculated by normalising the ratio of $\mathrm{CFSE}^{+}$targets vs. $\mathrm{CTO}^{+}$ control cells against CD1 KO mice. As shown in Figure 4.4, 10-25\% of splenocytes were killed in both the C57BL/6 or PKO mice. Although killing was low, it must be noted that among splenocytes B cells also express CD1d and can bind $\alpha$-GalCer, and could have been killed in an NKT cell specific manner. Therefore these results indicate that NKT cells could kill $\alpha$-GalCer loaded splenocyte targets in vivo. This is consistent with previously published data (360). However, these data also demonstrated that the mechanism of NKT cell killing was not dependent on perforin. Nonetheless, the data in Figures 4.3 and 4.4 fail to provide evidence for NKT cell-mediated killing of endogenous DC via a perforin-dependent mechanism. 
A.
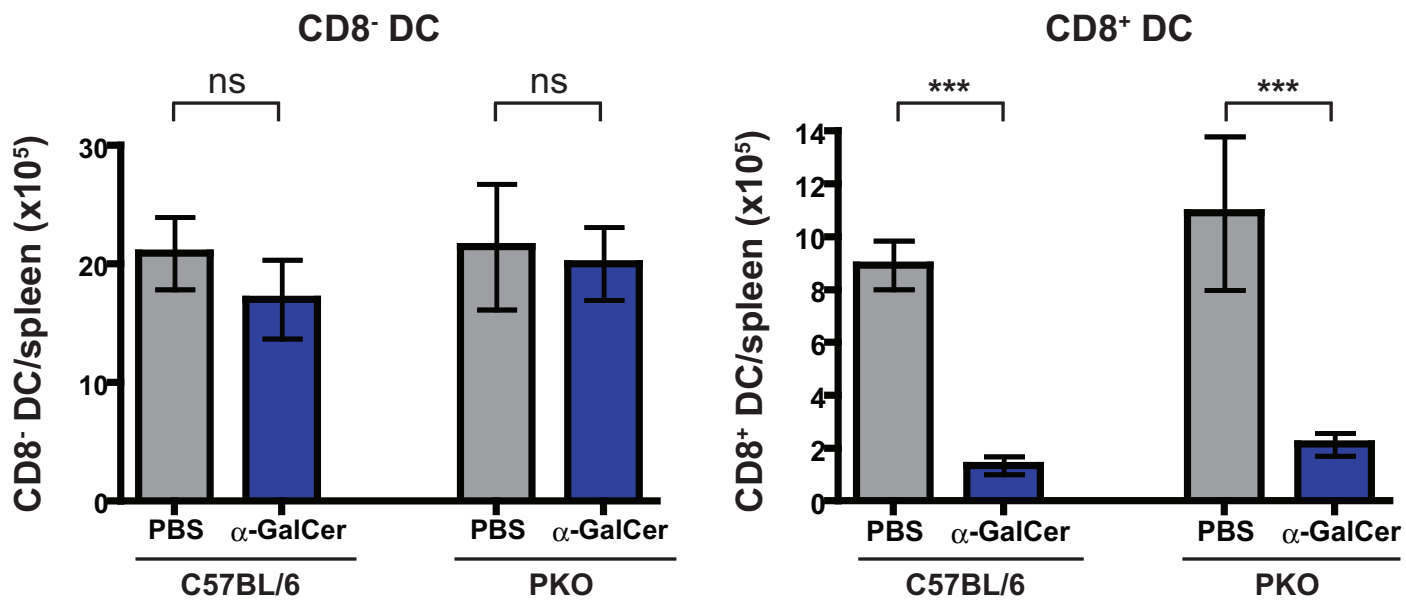

B.
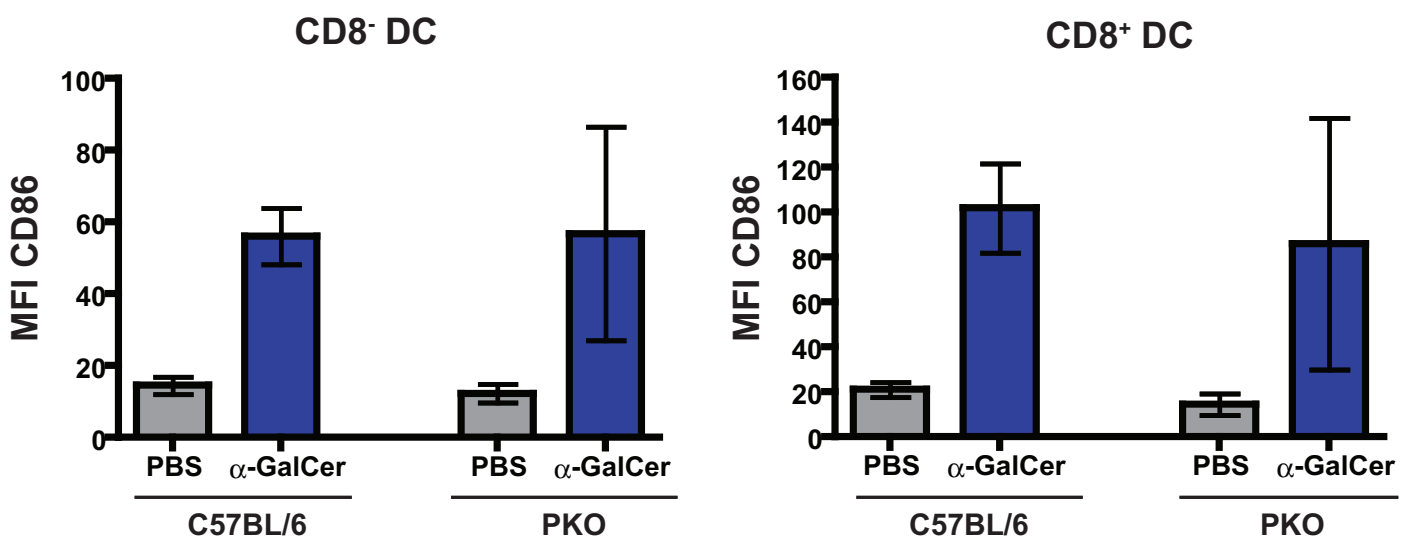

Figure 4.3 The decline in the $\mathrm{CDB}^{+} \mathrm{DC}$ subset after $\alpha$-GalCer treatment occurs in a perforinindependent manner.

C57BL/6 and PKO mice were injected i.v. with either $\alpha$-GalCer or PBS. Spleens were harvested 20 or $24 \mathrm{hr}$ later and analysed as described in Figure 4.1. (A) Total number of CD8 DC (left panel) and $\mathrm{CD} 8{ }^{+} \mathrm{DC}$ (right panel) present in the spleen $24 \mathrm{hr}$ after the indicated treatment. (B) The expression of CD86 on the CD8 ${ }^{-}$DC (left panel) and $\mathrm{CD}^{+}$DC (right panel) in the spleen $20 \mathrm{hr}$ after the indicated treatment. Each bar represents mean \pm SD of 3-4 mice per group.***: $p<0.001$, ns: not significant by one-way ANOVA with a Tukey post test. The experiment was repeated at least three times with similar results. 


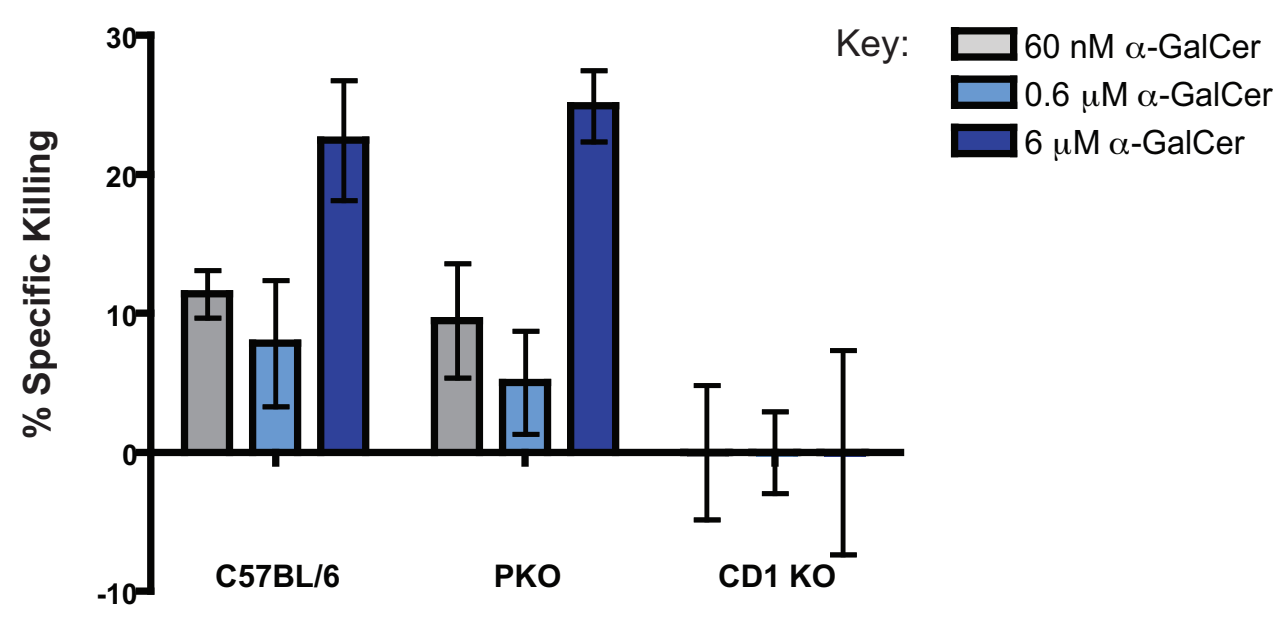

Figure 4.4 NKT cells can kill $\alpha$-GalCer pulsed targets in a perforin-independent manner.

Splenocytes from a C57BL/6 mouse were loaded with the indicated concentration of $\alpha$-GalCer or left untreated, and labelled with CFSE and CTO, respectively. A total of $2.5 \times 10^{6}$ labelled splenocytes were injected i.v. into C57BL/6, PKO and CD1 KO mice. MesLN were harvested $24 \mathrm{hr}$ after the adoptive transfer and analysed by flow cytometry for the presence of labelled target cells. The percentage of antigen-specific killing was calculated from the ratio of $\mathrm{CFSE}^{+}$targets vs. $\mathrm{CTO}^{+}$control cells and normalized against the CD1 KO mice. Bars represent mean \pm SD of 3-5 mice per group. 


\subsubsection{The decrease in $\mathrm{CD8}^{+} \mathrm{DC}$ is not due to a Fas-dependent mechanism}

As shown in Figure 4.3, the decline in $\mathrm{CD}^{+} \mathrm{DC}$ occurred in a perforin-independent manner. However, NKT cells also express FasL (152), which has been shown to be important for killing DP thymocytes (317) and certain tumour cell lines (315). To investigate if the reduction in $\mathrm{CD}^{+}$DC occurred in a Fas-FasL dependent manner, mice lacking expression of Fas (B6.lpr) were utilised. However, some reports have described that lpr mice have a deficiency in NKT cell numbers and function $(393,394)$, while another has reported no significant difference (395). Thus, before the B6.1pr mice could be used it was necessary to determine whether they had comparable numbers of NKT cells to C57BL/6 mice.

In preliminary experiments, the number of NKT cells in B6.lpr and C57BL/6 mice was assessed using a CD1d tetramer loaded with $\alpha$-GalCer in conjunction with an anti-CD3 mAb. The number of thymic NKT cells was comparable in C57BL/6 and B6.lpr mice, while a statistically significant increase in the number of spleen NKT cells was observed in B6.lpr mice compared to C57BL/6 mice (Figure 4.5). These results illustrated that the B6.lpr mice did not have a deficiency in the number of NKT cells. Thus further investigations were carried out using the B6.lpr mice. 
A.

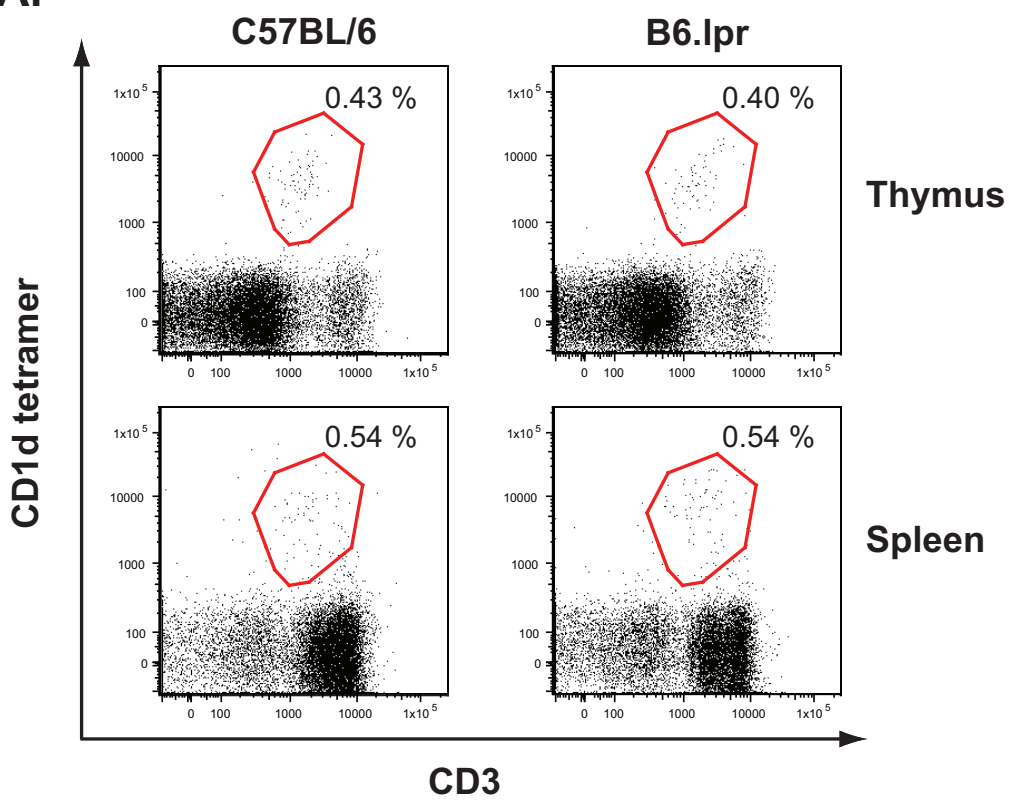

B.
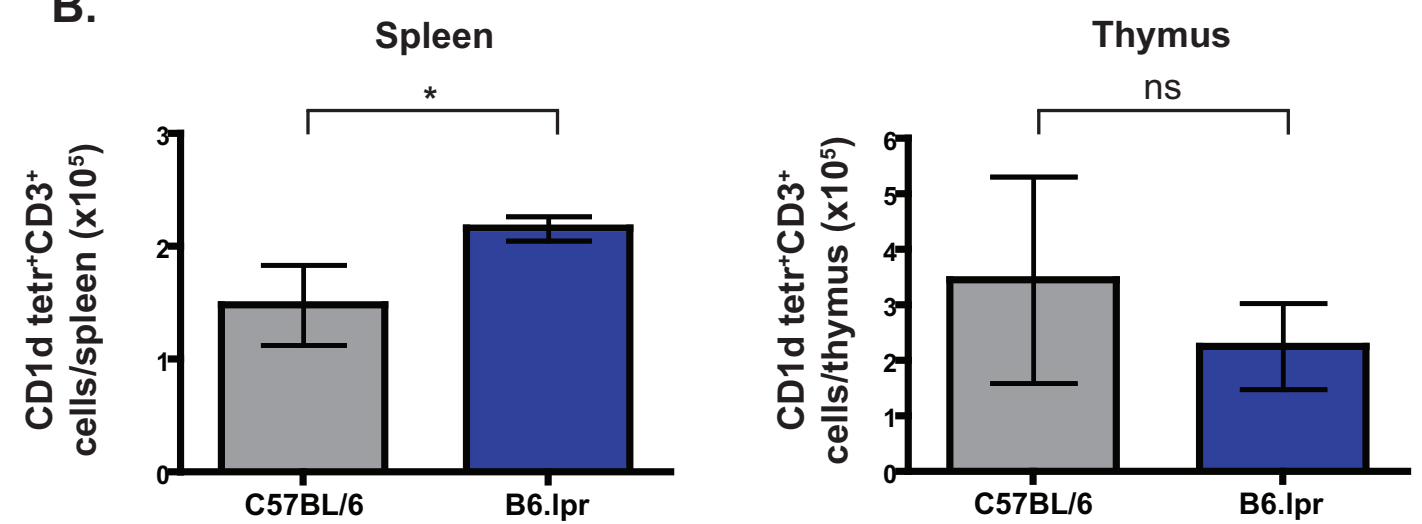

Figure 4.5 The number of NKT cells in the thymus and spleen of C57BL/6 and B6.lpr mice was comparable.

Spleens and thymi from untreated C57BL/6 and B6.lpr mice were harvested and processed into single cell suspensions. Cells were labelled with anti-CD3 mAb and CD1d tetramer loaded with $\alpha$-GalCer followed by examination of NKT cell numbers by flow cytometry. Dead $\left(\mathrm{DAPI}^{+}\right)$cells were excluded and lymphocytes were gated on the basis of their FSC vs. SSC properties. (A) Representative dot plots from 1 out of 3 mice show thymocytes (top) and splenocytes (bottom). $\mathrm{CD} 1 \mathrm{~d}$ tetramer ${ }^{+} \mathrm{CD} 3^{+}$cells are highlighted by polygonal gates, the percentage of these cells in the total gated population is indicated. (B) Total number of $\mathrm{CD} 1 \mathrm{~d}$ tetramer ${ }^{+} \mathrm{CD}^{+}$cells in the spleen (left) and thymus (right) from C57BL/6 and B6.lpr mice. Bars represent mean \pm SD of 3 mice per group. *: $p<0.05$ and ns: not significant by an unpaired Student's $t$ test. 
To establish whether the Fas-FasL pathway played a role in the decline of $C D 8^{+}$DC after treatment with $\alpha$-GalCer, C57BL/6 and B6.lpr mice were treated with $\alpha$-GalCer and $24 \mathrm{hr}$ later the spleens were harvested and the number of DC examined by flow cytometry. The number of $\mathrm{CD}^{+} \mathrm{DC}$ in B6.lpr mice treated with $\alpha$-GalCer was significantly decreased compared to the control group, though not as profoundly as in C57BL/6 mice (Figure 4.6 A). This would suggest that Fas played a small role in the decrease of the $\mathrm{CD} 8^{+} \mathrm{DC}$. The activation status of DC from C57BL/6 and B6.lpr mice was also assessed by the expression of cell surface marker CD86 (Figure 4.6 B). After $\alpha$-GalCer treatment DC from B6.lpr mice did not significantly upregulate expression of CD86 compared to the PBS treated group. This was in contrast to the C57BL/6 mice, in which a significant increase in the expression of CD86 was observed after $\alpha$-GalCer treatment (Figure 4.6 B). Thus it would appear that Fas signalling is important for the activation of $\mathrm{CD}^{+} \mathrm{DC}$, but has only a small effect on the loss of $\mathrm{CD} 8^{+} \mathrm{DC}$ in the spleen after $\alpha-\mathrm{GalCer}$ treatment. 
A.

\section{CD8- DC}

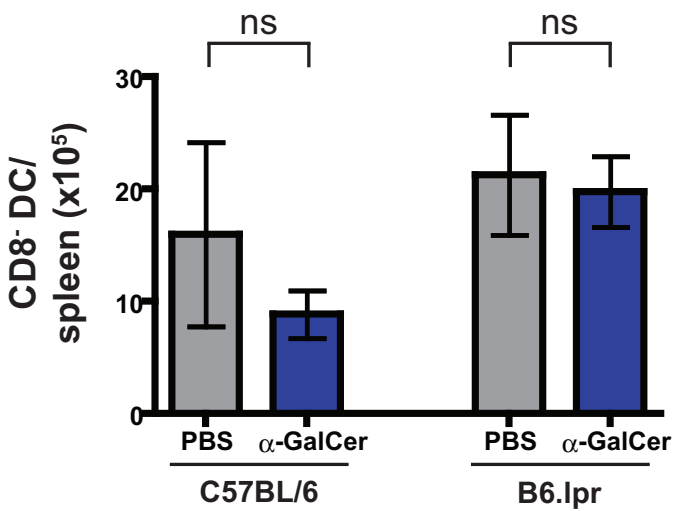

B.

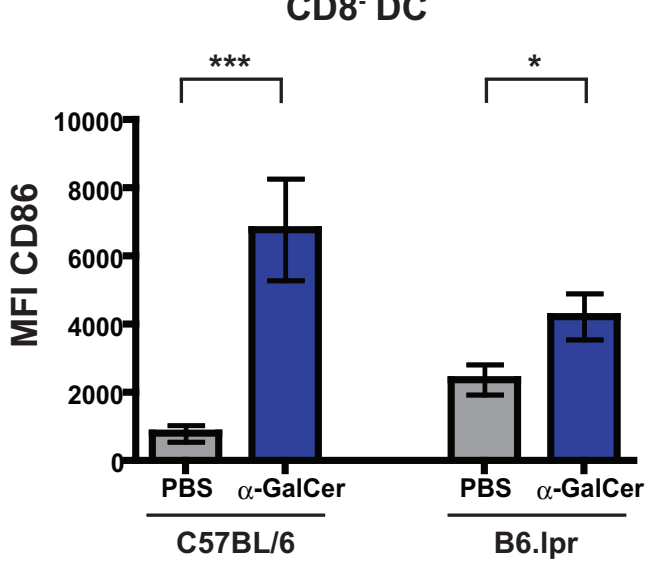

CD8+ DC

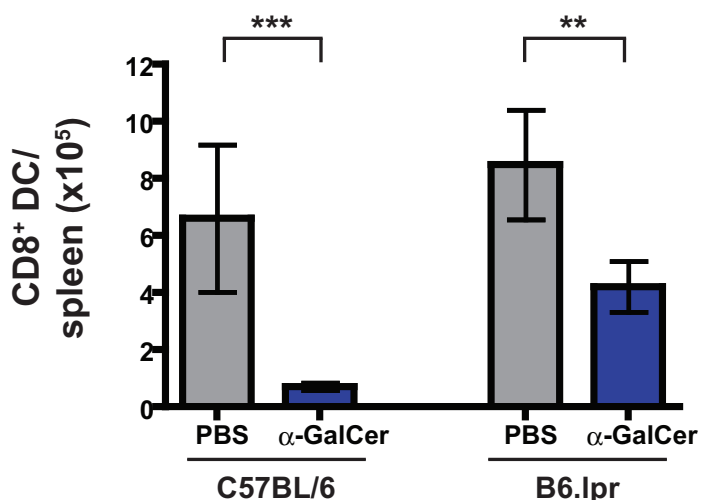

CD8 ${ }^{+} \mathrm{DC}$

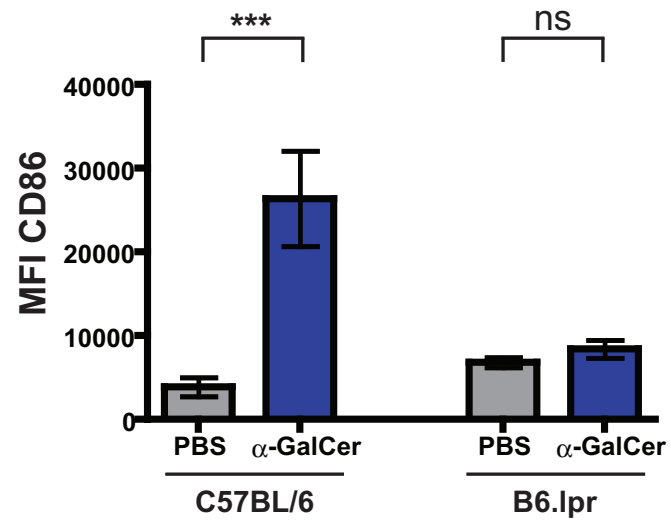

Figure 4.6 Fas deficiency has a minor effect on the loss of $\mathrm{CD8}^{+} \mathrm{DC}$ after $\alpha$-GalCer treatment.

C57BL/6 and B6.lpr mice were injected i.v. with either PBS or $\alpha$-GalCer. Spleens were harvested 24 hr later and analysed as described in Figure 4.1. (A) Total number of CD8 ${ }^{-}$DC (left panel) and CD8 ${ }^{+}$ DC (right panel) present in the spleen $24 \mathrm{hr}$ after the indicated treatment. (B) The expression of CD86 on the CD8 ${ }^{-}$DC (left panel) and CD8 ${ }^{+}$DC (right panel) in the spleen $24 \mathrm{hr}$ after the indicated treatment. Bars represent mean \pm SD of 5 mice per group. $* * *: p<0.001, * *: 0.001<p<0.01, *: p<$ 0.05 , and ns: not significant by one-way ANOVA with a Tukey post test. 


\subsubsection{Assessing the ova-specific $\mathrm{CD8}^{+} \mathrm{T}$ cell response after treatment with $\alpha$-GalCer and ova protein}

In some infection models PKO mice have been reported to generate an enhanced immune response $(338,339,374-376,378)$. Therefore, it was of interest to investigate whether there was a difference in the percentage of antigen-specific $\mathrm{CD}^{+} \mathrm{T}$ cells after treatment with $\alpha$ GalCer and ova protein. Seven days after $\alpha$-GalCer and ova protein administration, CD ${ }^{+} \mathrm{T}$ cells specific for the dominant CD8 epitope, ova $257-264$ were detected in the blood using an MHC I pentamer by flow cytometry (Figure 4.7). This day 7 time point was chosen based on previous studies using the same $\alpha-G a l C e r$ and ova model (320). No statistical difference in the percentage of $\mathrm{CD}^{+} \mathrm{K}^{\mathrm{b}}$-ova $257-264{ }^{+} \mathrm{T}$ cells was found between C57BL/6 and PKO mice (Figure 4.7 B). These data suggest that in this model the PKO mice do not generate an enhanced CD ${ }^{+}$ $\mathrm{T}$ cell response in response to ova administered with $\alpha$-GalCer. 
Figure 4.7 C57BL/6 and PKO mice treated with $\alpha$-GalCer and ova protein generate comparable ova-specific $\mathrm{CDB}^{+} \mathrm{T}$ cell responses.

C57BL/6 and PKO mice were injected i.v. with $\alpha$-GalCer and ova protein and seven days later blood was collected from the tail vein and examined by flow cytometry for the presence of $\mathrm{CD} 8^{+} \mathrm{K}^{\mathrm{b}}$-ova 257 ${ }_{264}{ }^{+} \mathrm{T}$ cells. Dead $\left(\mathrm{PI}^{+}\right)$cells were excluded and lymphocytes were gated on the basis of their FSC vs. SSC properties. (A) Panels show dot plots of $\mathrm{CD} 8^{+}$cells, the oval highlights the $\mathrm{CD} 8^{+} \mathrm{K}^{\mathrm{b}}$-ova $257-264^{+}$ cells. The percentage of these cells in the total gated population is indicated. (B) Percentages of $\mathrm{CD}^{+} \mathrm{K}^{\mathrm{b}}$-ova $257-26{ }^{+} \mathrm{T}$ cells seven days after administration of $\alpha$-GalCer and ova protein. Each point represents an individual mouse and the lines represent the mean. $* *: 0.001<p<0.01$ and ns: not significant by one-way ANOVA with a Tukey post test. 
A.

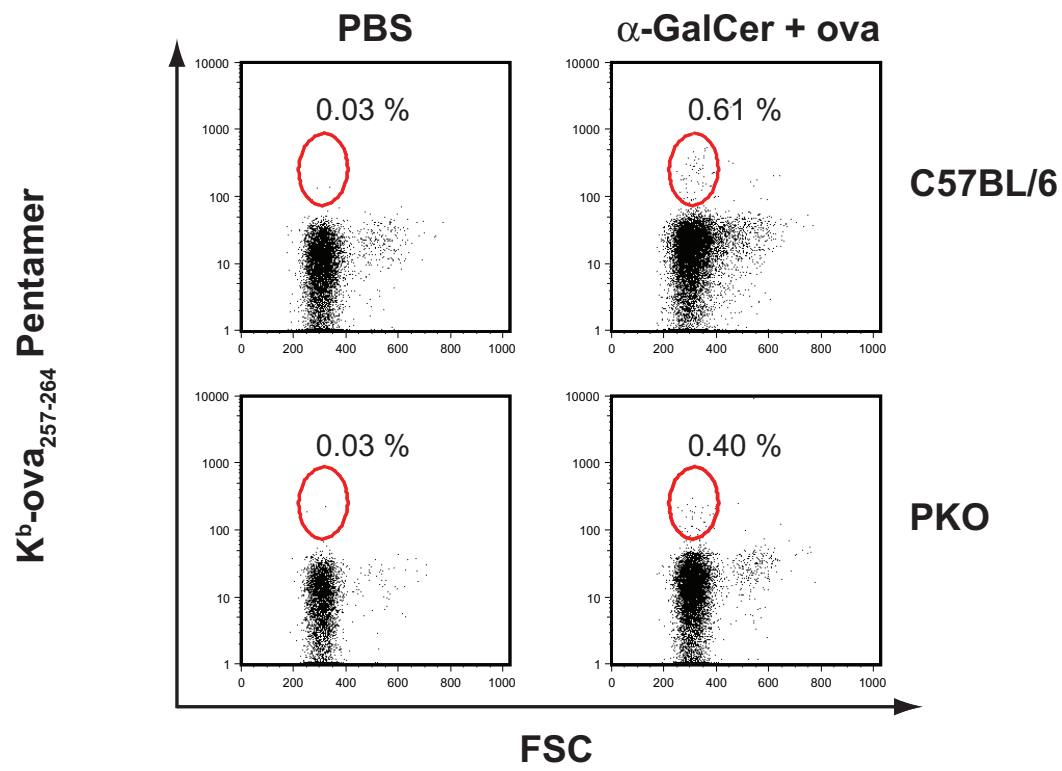

B.

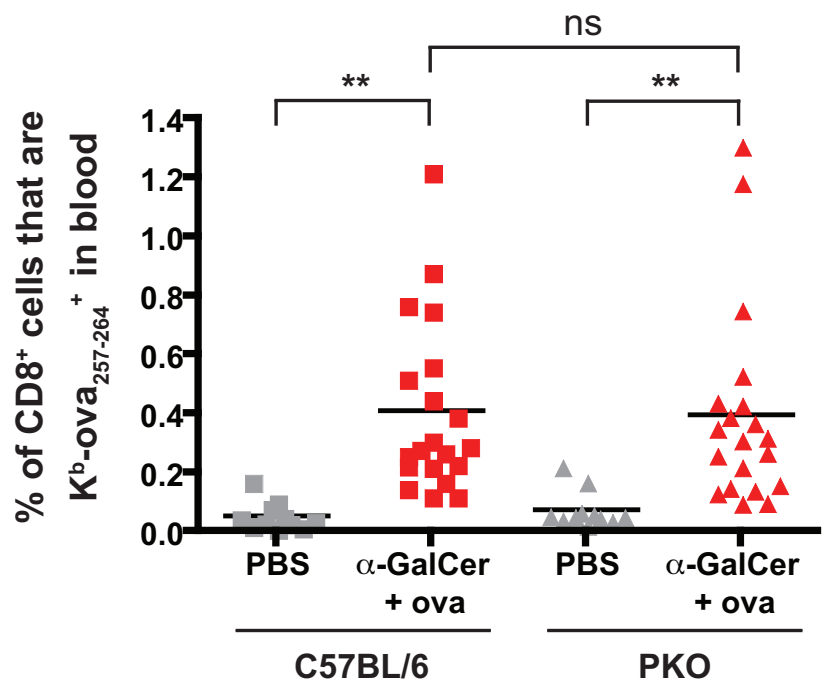




\subsubsection{Assessing DC survival after a boost of ova protein}

So far experiments did not reveal perforin-mediated DC elimination during a primary ovaspecific $\mathrm{CD}^{+} \mathrm{T}$ cell response. However, as discussed in chapter 1, there is evidence indicating that memory $\mathrm{CD}^{+} \mathrm{T}$ cells can eliminate DC. To determine whether DC might be eliminated during a secondary ova-specific $\mathrm{CD}^{+} \mathrm{T}$ cell response, mice were primed with $\alpha$-GalCer and ova protein as described previously, followed by an i.v. injection of ova protein 30 days after the original treatment. Initially the ova-specific $\mathrm{CD} 8^{+} \mathrm{T}$ cell response was assessed to determine whether a secondary $\mathrm{T}$ cell response was being generated using this boosting method. There was no clear increase in the percentage of $\mathrm{CD}^{+} \mathrm{K}^{\mathrm{b}}$-ova $257-264{ }^{+} \mathrm{T}$ cells in mice that received the secondary ova injection compared to those that only received the primary treatment (Figure 4.8), indicating that a secondary immune response was not induced.

Although no secondary ova-specific T cell response was detected, two and seven days after the boost of ova protein the total number of $\mathrm{DC}\left(\mathrm{CD} 11 \mathrm{c}^{+} \mathrm{MHC} \mathrm{II}^{+}\right)$and $\mathrm{CD} 8^{+} \mathrm{DC}$ was assessed in the spleen (Figure 4.9). At both time points no significant difference was observed in total number of DC or $\mathrm{CD} 8^{+} \mathrm{DC}$ in C57BL/6 mice across all groups (Figure 4.9). Similar results were also observed in PKO mice (Figure 4.9). These data indicate that in the absence of a secondary ova-specific T cell response DC are not eliminated. 


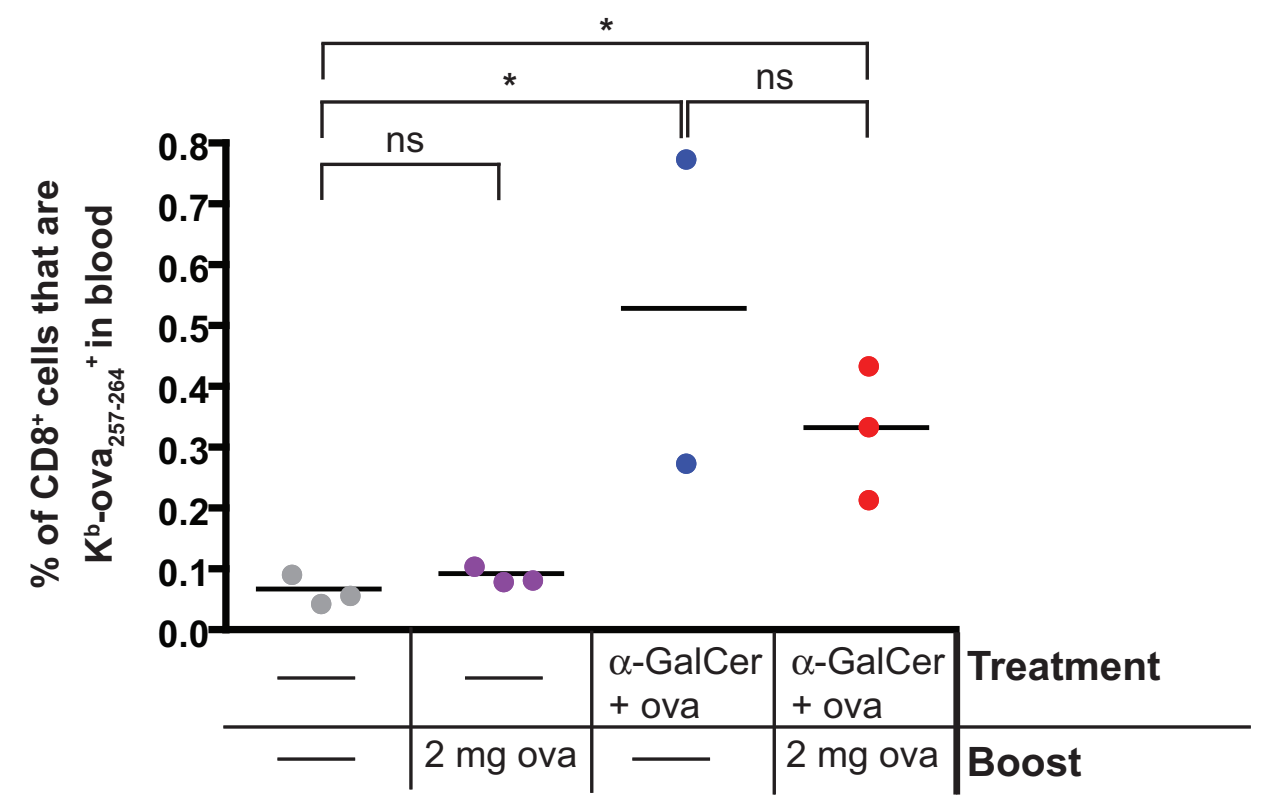

Figure 4.8 A boost of ova protein did not induce an increased percentage of $\mathrm{CDB}^{+} \mathrm{K}^{\mathrm{b}}-\mathrm{ova}_{257-264}{ }^{+} \mathrm{T}$ cells.

C57BL/6 mice were injected i.v. with $\alpha$-GalCer and ova protein or left untreated. Mice were then treated 30 days later with $2 \mathrm{mg}$ ova protein i.v. or left untreated. Blood was collected five days after boosting and analysed as described in Figure 4.7. The percentages of $\mathrm{CD} 8{ }^{+} \mathrm{K}^{\mathrm{b}}$-ova $257-264{ }^{+}$cells are shown. Each point represents one individual mouse and lines represent the mean. $*: p<0.05$, and ns: not significant by one-way ANOVA with a Tukey post test. 
A.

Day 2 after Boost

CD11C+MHC II+ DC

CD8 ${ }^{+} \mathrm{DC}$
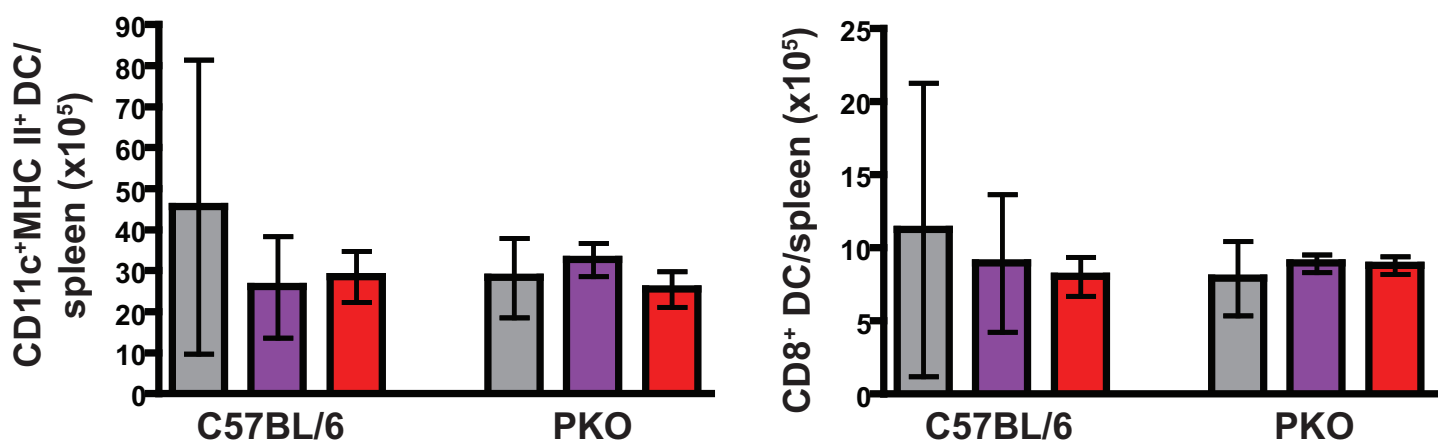

B.

Day 7 after Boost

CD11 + MHC II+ DC
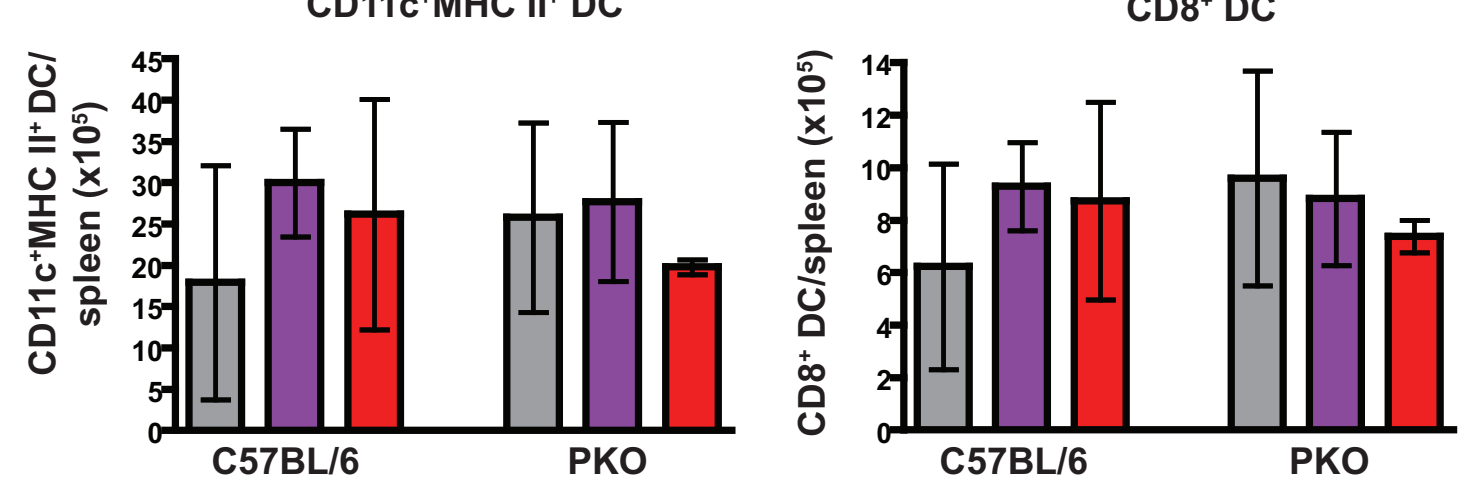

Key: \begin{tabular}{l|l} 
Treatment & Boost \\
\hline & $\begin{array}{l}\text { PBS } \\
\text { PBS }\end{array}$ \\
\hline a-GalCer + ova & ova \\
\hline
\end{tabular}

Figure 4.9 Boosting with ova protein does not lead to a change in the number of spleen DC.

C57BL/6 and PKO mice were injected i.v. with either PBS or $\alpha$-GalCer and ova protein. Mice were boosted 30 days later with either an i.v. injection of PBS or $200 \mu \mathrm{g}$ ova protein. Then two (A) or seven days (B) after the boost of ova protein the spleens were harvested and the number of CD11 $\mathrm{c}^{+} \mathrm{MHC} \mathrm{II}^{+}$ and $\mathrm{CD}^{+} \mathrm{DC}$ were analysed as described in Figure 4.1. Bars represent mean \pm SD of 3-4 mice per group. No statistical differences between groups were found using a one-way ANOVA. 


\subsubsection{IgG1 levels, and number of basophils are similar in PKO and C57BL/6 mice}

While endeavouring to induce a secondary ova-specific $\mathrm{T}$ cell response it was observed that after the boost of ova protein all the mice became unresponsive within seconds. These symptoms are suggestive of systemic anaphylactic shock, which is a rapid-onset, life threatening allergic reaction $(396,397)$. Of interest PKO mice were more severely affected by the boost of ova protein than C57BL/6 mice, as PKO mice never recovered from the state of unresponsiveness and had to be culled for ethical reasons. Thus, it was of interest to investigate why PKO mice reacted more severely than $\mathrm{t}$ C57BL/6 mice to the second dose of ova protein.

It is well known that IgE is crucially involved in systemic anaphylaxis (398) and more recently IgG1 has been shown to also play an important role (399-401). The more severe anaphylactic-like symptoms observed in PKO mice might have been due to an increased amount of circulating ova-specific antibodies. To assess the level ova-specific IgG1 and IgE, ELISAs were performed using serum samples collected 30 days after the $\alpha$-GalCer and ova protein treatment. This time point was chosen because this was when the boost of ova protein had been administered. No statistically significant difference in the serum levels of ovaspecific IgG1 was found between the PKO mice and C57BL/6 mice (Figure 4.10). The levels of ova-specific IgE in both C57BL/6 and PKO mice treated with $\alpha$-GalCer and ova protein were greater than in the PBS treated mice but still very low and below the level of sensitivity of the assay. Thus these data suggest that the observed increased unresponsiveness in the PKO mice was not directly related to an increase in ova-specific antibodies. 


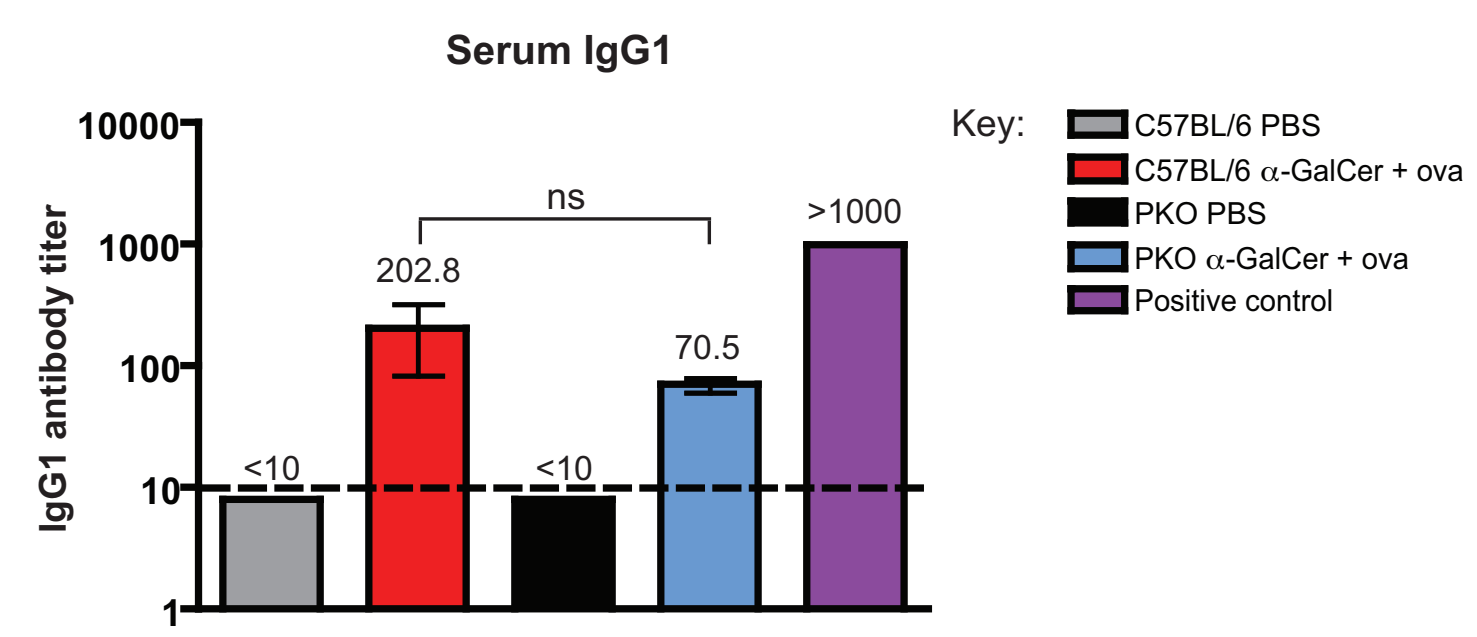

Figure 4.10 The levels of ova-specific IgG1 in the serum of C57BL/6 and PKO mice were similar. C57BL/6 and PKO mice were injected i.v. with $\alpha$-GalCer and ova protein or PBS. Serum was collected 30 days later via cardiac puncture. To generate a positive control mice were treated twice i.p. with alum and ova protein (see methods). ELISA specific for ova IgG1 was performed. Each bar represents mean \pm SEM of 4-5 mice per group. Numbers represent the mean antibody titer. The PBS groups are marked $<10$ as the antibody titer was below the detection limit of the assay, whereas the positive control is marked as $>1000$ as the antibody titer was greater than the highest serum dilution. The dotted line indicates the detection limit. ns: not significant by an unpaired Student's $t$ test. The experiment was repeated three times with similar results. 
The severity of systemic anaphylactic shock is also related to the number of cells present that mediate the symptoms. Recently, it was discovered that basophils are important in IgGmediated anaphylaxis (402). Unlike mast cells that reside in tissue, basophils are found in the bloodstream (403). Since the ova protein was administered i.v., it was more likely that the boost of ova protein would be more accessible to basophils than mast cells. Consequently, the number of basophils was investigated to verify if this accounted for the increased unresponsiveness observed in the PKO mice. Mice were bled 30 days after $\alpha$-GalCer and ova protein treatment, and basophils were identified as $\mathrm{CD} 45^{\text {int }} \mathrm{Fc \varepsilon R} 1^{+}$by flow cytometry (Figure 4.11 A). The percentage of basophils in the blood of PBS treated mice was approximately 0.3 $\%$ and this corresponded to previously published work (403). However, the percentage of basophils did not change after treatment with $\alpha$-GalCer and ova protein (Figure $4.11 \mathrm{~B}$ ). Furthermore, PKO mice did not have an increased number of basophils compared to C57BL/6 mice (Figure $4.11 \mathrm{~B}$ ). This result indicated that an increased percentage of basophils was not the explanation for the increased mortality observed in PKO mice. 
A.
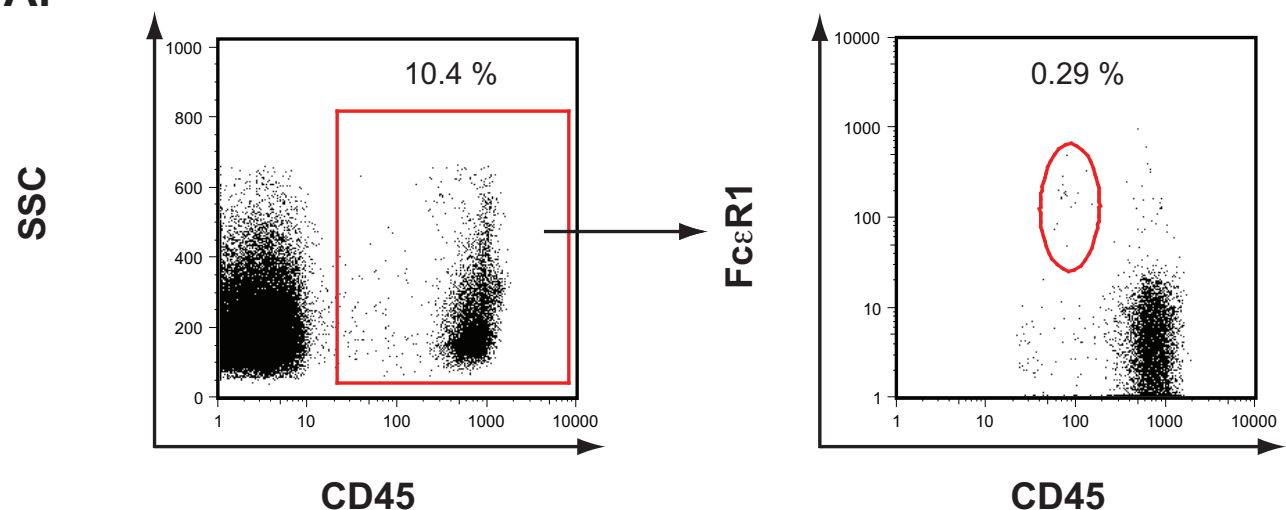

B.

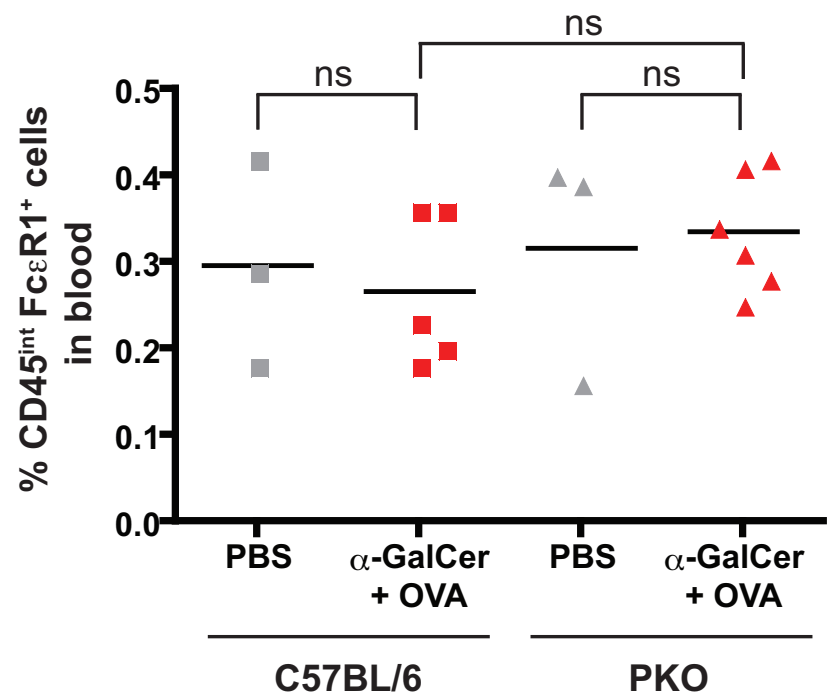

Figure 4.11 C57BL/6 and PKO mice show a similar percentage of basophils present in the peripheral blood after treatment with $\alpha$-GalCer and ova protein.

C57BL/6 and PKO mice were injected i.v. with $\alpha-G a l C e r$ and ova protein or PBS. Blood was collected 30 days later and analysed by flow cytometry for the percentage of basophils. Dead ( $\left.\mathrm{PI}^{+}\right)$cells were excluded and lymphocytes were gated on the basis of their FSC vs. SSC properties. (A) Representative dot plots show $\mathrm{CD} 45^{+}$cells in the rectangle gate and $\mathrm{CD} 45^{\mathrm{int}} \mathrm{Fc \varepsilon RI}{ }^{+}$basophils are indicated by an oval, the percentage of these cells in the total gated population is indicated. (B) Percentage of basophils in peripheral blood after the indicated treatment. Each point represents an individual mouse and lines represent the mean. ns: not significant by one-way ANOVA. 


\subsection{Discussion}

The aim of this chapter was to determine whether DC survival was regulated in a perforindependent manner after treatment with $\alpha$-GalCer and ova protein. The use of $\alpha$-GalCer and ova protein enabled the assessment of both NKT cells and $\mathrm{CD} 8^{+} \mathrm{T}$ cells in the role of regulating DC survival. Treatment with $\alpha$-GalCer and ova protein led to a decrease only in the $\mathrm{CD} 8^{+} \mathrm{DC}$ subset, which did not require the presence of ova-specific $\mathrm{CD} 8^{+} \mathrm{T}$ cells, and thus is likely to be mediated exclusively by NKT cells. The disappearance of $\mathrm{CD} 8^{+} \mathrm{DC}$ occurred in a perforin- and Fas-independent manner.

Similar to CTL, NKT cells are capable of cytotoxic function. NKT cells express perforin and the ligands for a number of cell death receptors such as FasL and TRAIL (152, 235, 311-313, 318). Perforin-, FasL- and TRAIL-mediated cytotoxicity by human and mouse NKT cells have been demonstrated to be important for killing DC and tumour cell lines (152, 310, 312$315,318)$. However, during this study it was found that after $\alpha$-GalCer treatment the decline in $\mathrm{CD}^{+} \mathrm{DC}$ occurred in a perforin-independent manner. Furthermore, experimental evidence suggests that the loss of splenic $\mathrm{CD}^{+} \mathrm{DC}$ was independent of Fas expression. An earlier report showed that mice lacking Fas expression had reduced NKT cells numbers and function (394). Contrary to this, results in this chapter confirmed that the B6.lpr used in this thesis had comparable numbers of $\alpha$-GalCer reactive NKT cells to C57BL/6 mice, which was also supported by another published study that assessed the percentage of NK1.1 ${ }^{+} \mathrm{TCR} \alpha \beta^{+} \mathrm{NKT}$ cells in the spleen (395). However, functional capacity of the NKT cells from B6.lpr mice used in this thesis was not assessed. Since the study which found reduced functional capacity correlated this with a reduced number of NKT cells (394), it is likely that the NKT cells from the B6.lpr mice are functionally equivalent to those from C57BL/6 mice. As it would appear that neither perforin nor FasL directly mediated the decrease of the $\mathrm{CD} 8^{+} \mathrm{DC}$ it would suggest that the decrease was not mediated by direct interaction with the NKT cell. However, other death receptors such as TRAIL would need to be investigated before this can be formally concluded. 
It has been reported that murine DC are resistant to Fas induced apoptosis (404). This resistance is due to an increased expression of the molecule FLIP, which inhibits apoptosis induced by both Fas and TNFR ligation (404). However, in an earlier study, blood derived human DC were sensitive to Fas-induced apoptosis unless they received signalling through CD40 (146). CD40 ligation of DC has been shown to enhance DC survival $(123,128,147)$. It is thought that this enhanced survival is partly due to the DC upregulating anti-apoptotic proteins such as Bcl-xL (132). NKT cells express CD40L upon activation (122) and interact with CD40 on the DC after $\alpha$-GalCer treatment $(122,187,320,405)$. Therefore it would be expected that CD40 ligation would be occurring in the model presented in this thesis and that the DC would upregulate anti-apoptotic proteins leading to enhanced cell survival. This is in contrast to the observed decline in $\mathrm{CD}^{+} \mathrm{DC}$ after $\alpha$-GalCer treatment. However, it is possible that some protection is being mediated by CD40 ligation as well as resistance to Fas mediated cell death by the upregulation of FLIP. Nevertheless, this protection is not sufficient to prevent a significant decrease in the $\mathrm{CD} 8^{+} \mathrm{DC}$ subset of the spleen from occurring.

The results described in this chapter suggest that presence of CTL did not cause the observed loss of DC that occurred after treatment with $\alpha$-GalCer and ova protein. However, memory $\mathrm{CD}^{+} \mathrm{T}$ cells have also been shown to eliminate DC $(156,169,344)$; thus a secondary ova response was also studied. To assess this, mice were treated with $\alpha$-GalCer and ova protein followed by administration of a second dose of ova protein 30 days later. $\alpha$-GalCer was not administered during the boost as it has been reported that NKT cells become hyporesponsive after an initial treatment of $\alpha$-GalCer $(406,407)$. However, boosting with ova only did not successfully generate a secondary ova-specific $\mathrm{T}$ cell response. This could be due to the presence of ova-specific antibodies clearing the antigen. Consequently, a different system is required to create secondary ova-specific $\mathrm{T}$ cell response to evaluate whether ova-specific memory CTL regulate DC survival. The use of $\alpha$-GalCer and ova protein followed by treatment with recombinant vaccinia virus expressing full length ova protein is an effective method to prime and boost ova-specific $\mathrm{CD} 8^{+} \mathrm{T}$ cell response (320) and would be a potential model system to evaluate DC survival in the presence of ova-specific memory CD8 ${ }^{+} \mathrm{T}$ cells. 
After the second dose of ova protein $(2 \mathrm{mg})$ the mice became unresponsive within minutes and did not recover for a long time. The rapidity of this response suggested an antibody-mediated response. An IgE-mediated type I immediate hypersensitivity reaction can occur within minutes of allergen exposure (408). Systemic anaphylaxis is a type I immediate hypersensitivity where IgE bound to FceRI on mast cells and basophils is cross-linked by allergen, resulting in the release of chemical mediators such as histamine and release platelet activating factor (PAF). Release of these chemical mediators ultimately leads to the characteristic symptoms of anaphylaxis (408), such as hypotension, hypothermia, decreased mobility, and scratching (400). An assessment criteria has been described to quantitatively assess the anaphylactic symptoms with a score 0 indicating no symptoms and a score 5 indicating death. According to this assessment criteria, mice injected with the second dose of ova protein immediately went to a score 4 which is classified as no activity after prodding or tremor and convulsion (409). Therefore, rather than the intended secondary ova-specific CD8 ${ }^{+}$ $\mathrm{T}$ cell response, the second injection of ova protein resulted in symptoms with characteristics similar to systemic anaphylaxis.

Administration of $\alpha$-GalCer leads to B cell activation and antibody secretion (327-331, 333335). Mice treated with particulate $\alpha$-GalCer produced IgM and early class switched specific antibodies (333). Additionally, systemic treatment with $\alpha$-GalCer or ova in Complete Freund's Adjuvant has previously been found to lead to high levels of total and ova-specific IgE compared to control (332). Furthermore, a report that gave repeated low doses of $\alpha$ GalCer and protein followed by a recall treatment of protein alone weeks later showed that the specific Ig titer was enhanced in the recall response compared to protein alone (335). In this thesis antibody titers were also measured in mice treated with $\alpha$-GalCer and ova protein and found the presence of ova-specific IgG1 and low levels of IgE after treatment with $\alpha$-GalCer and ova protein. Taken together these results might account for why the mice pre-treated with $\alpha$-GalCer and ova protein became unresponsive upon administration of a second dose of ova protein.

It was observed that PKO mice reacted more severely to the second dose of ova protein compared to C57BL/6 mice, as none of the PKO mice recovered. This suggests that perforin 
might play a role in the development of the observed anaphylactic shock-like symptoms, which is a novel finding. As the level of $\operatorname{IgE}$ and $\operatorname{IgG} 1$ has been directly correlated with the severity of the anaphylactic shock (410), it was asked if PKO mice had higher serum antibody titers. PKO mice have already been reported to generate a stronger antibody response compared to C57BL/6 mice (375). Investigations into the levels of ova-specific $\operatorname{IgE}$ and $\operatorname{IgG} 1$ found no evidence that PKO mice had an enhanced level of ova-specific antibodies present in the serum compared to C57BL/6 mice. However, the ELISA technique used to determine the level of ova-specific antibodies, only measures free circulating antibodies present in the serum and not cell bound antibodies. Though, 30 years ago a correlation between serum IgE levels and the amount of $\operatorname{IgE}$ bound to basophils was observed (411). More recently, in vivo studies have shown that IgE increases the cell surface expression of FceRI on basophils (412), indicating that $\operatorname{IgE}$ itself is a major regulator of the amount of cell surface bound $\operatorname{IgE}$. Thus it is unlikely that PKO mice would have an increased level of cell surface bound IgE if they did not have a significant increase in serum IgE. Therefore, increased levels of ova-specific antibodies in PKO mice do not appear to account for the increased reaction to the second dose of ova protein observed in these mice.

Severity of anaphylactic shock is also influenced by the number of mast cells, macrophages and basophils present (400). Basophils have recently been shown to be important in IgG mediated anaphylaxis (402). IgG mediated anaphylaxis closely resembles the classical pathway that involves mast cells and IgE antibodies (400). In allergen-sensitised mice, antigen-specific IgG antibodies form antigen-IgG immune complexes that are immediately captured by circulating basophils through $\mathrm{Fc}$ receptors for $\operatorname{IgG}(\mathrm{Fc} \gamma \mathrm{Rs})$. Basophils become activated by the immune complexes and PAF, which in turn increases vascular permeability by acting on endothelial cells, thereby leading to systemic anaphylaxis (413). Thus an increased number of basophils in PKO mice could have explained the enhanced severity in anaphylaxis due to increased degranulation and presence of chemical mediators. However, a similar percentage of basophils was observed in both C57BL/6 and PKO mice. Further investigations into the effector functions of the basophils from the two mouse strains would need to be performed to establish whether increased basophil reactivity might be a mechanism for the enhanced anaphylaxis in PKO mice. One approach to examine this would be to use a 
PAF antagonist to assess the role of PAF released by basophils and whether this differs between WT and PKO mice. Additionally, a mAb has recently been described that binds to CD200R3 and upon in vivo injection it causes the depletion of basophils from the peripheral blood and spleen of mice $(414,415)$. Thus, this depleting antibody could be used in combination with PKO mice to assess whether basophils play a role in the generation of the observed severe anaphylactic reaction.

It was not determined which pathway of anaphylaxis was occurring, either $\operatorname{IgE}$ or $\operatorname{IgG}$ mediated or a combination of the two, therefore investigations into other cell types such as mast cells may provide evidence and clarify why PKO mice presented with increased anaphylactic shock-like symptoms. In addition, although this study observed that PKO mice had a more severe reaction to the second dose of ova protein this was not assessed in a quantitative manner. Thus before further analysis is performed to evaluate whether PKO mice respond differently to the second dose of ova protein compared to C57BL/6 mice a quantitative method needs to be established, such as measuring changes in body temperature or measuring the levels of serum derived factors.

\subsubsection{Summary}

This chapter reports that treatment with $\alpha$-GalCer and ova protein induced a decrease in the number of splenic $\mathrm{CD} 8^{+} \mathrm{DC}$. Through the use of knockout mice it was shown that the mechanism of this decline was independent of both perforin and Fas. NKT cells, which become activated in the presence of $\alpha$-GalCer, are the likely mediators of this decrease in $\mathrm{CD}^{+}$DC. Responses induced with $\alpha$-GalCer and ova protein could not be boosted in vivo using a second dose of ova protein, thus preventing the study of DC elimination during a secondary ova-specific $\mathrm{CD}^{+} \mathrm{T}$ cell response. Instead, the second dose of ova protein elicited a response that shared some characteristics of anaphylaxis that could be due to the presence of ova-specific antibodies generated after the initial treatment with $\alpha$-GalCer and ova protein. Upon treatment with the second dose of ova protein it was also observed that PKO mice 
reacted more severely than C57BL/6 mice. This would be an interesting area for future studies as a role for perforin in anaphylaxis has not been previously demonstrated. 


\section{Chapter 5:}

\section{Study of the mechanism of $\mathrm{CD8}^{+} \mathrm{DC}$ loss after treatment with $\alpha$-GalCer}




\subsection{Introduction}

It has been proposed that DC killing is one of the immune regulatory functions of NKT cells, to prevent excessive antigen stimulation $(151,152)$ and to regulate the Th1/Th2 balance by removing DC that are a major source of IL-12 (310). However, it is yet to be determined whether NKT cells mediate DC killing in vivo, and how.

Results described in Chapter 4, demonstrated that after $\alpha$-GalCer treatment, loss of DC occurred in a perforin- and FasL-independent manner, suggesting that NKT cells must use a different mechanism to mediate loss of DC. NKT cells express a wide variety of cell death-inducing effector molecules that could contribute to DC killing; as well as perforin $(235,311,313)$ and FasL $(152,317)$ they express TRAIL (318). Furthermore, NKT cells also secrete the cytokines, IFN- $\gamma$ and TNF- $\alpha$ that could contribute to cell lysis $(266,270)$. TNF- $\alpha$ binds its receptor to activate the classical caspase dependent apoptosis pathway, whereas IFN- $\gamma$ can promote $\mathrm{NK}$ and $\mathrm{CD}^{+} \mathrm{T}$ cell activation resulting in cell lysis. This chapter assessed whether IFN- $\gamma$ and TNF- $\alpha$ are involved in NKT cell-mediated killing of DC.

Experiments described in Chapter 4 showed that 24-48 hr after i.v. injection with $\alpha$-GalCer the splenic $\mathrm{CD}^{+} \mathrm{DC}$ subset was reduced, whereas no change in number of the $\mathrm{CD}^{-} \mathrm{DC}$ was observed. The splenic $\mathrm{CD} 8^{+}$and $\mathrm{CD} 8^{-} \mathrm{DC}$ are distinct DC subsets as they have differential gene expression (64), are found in different anatomical locations $(44,61,62)$ and the $\mathrm{CD}^{+}$DC preferentially secrete more $\mathrm{IL}-12(44,63)$. The $\mathrm{CD} 8^{+} \mathrm{DC}$ subset has been found to be important for $\mathrm{CD}^{+} \mathrm{T}$ cell mediated immunity. $\mathrm{CD} 8^{+} \mathrm{DC}$ are more efficient at cross presenting antigen than the CD8 ${ }^{-}$DC subset $(100,101)$ and they are the principle DC in priming naïve $\mathrm{CD}^{+} \mathrm{T}$ cell during viral infections (416). Recently, selective depletion of the $\mathrm{CD} 8^{+} \mathrm{DC}$ was reported to profoundly abrogate $\mathrm{CD} 8^{+} \mathrm{T}$ cell proliferation and reduced subsequent tumour challenge (417). Additionally, a role in tolerance induction has been proposed, as $\mathrm{CD}^{+} \mathrm{DC}$ selectively phagocytose apoptotic cells $(102,418,419)$ and decreased numbers of $\mathrm{CD}^{+} \mathrm{DC}$ led to reduced tolerance induction resulting in increased onset of experimental autoimmune encephalomyelitis (420). Therefore, the lack of this specific DC subset after $\alpha$-GalCer treatment could impact on subsequent immune responses. Thus it was of particular interest to determine the mechanism of DC loss after $\alpha$-GalCer treatment. 


\subsection{Aims}

The experiments described in this chapter were designed to identify the mechanism of the loss of $\mathrm{CD}^{+} \mathrm{DC}$ after treatment with $\alpha$-GalCer, and to assess whether the decrease in $\mathrm{CD} 8^{+} \mathrm{DC}$ affected immune responses against protein antigen.

The specific aims were:

- To characterise the kinetics of the decrease in the $\mathrm{CD} 8^{+} \mathrm{DC}$ subset.

- To determine if the loss of $\mathrm{CD}^{+} \mathrm{DC}$ required cognate interaction with NKT cells.

- To determine whether the decline in $\mathrm{CD}^{+} \mathrm{DC}$ after $\alpha$-GalCer and ova protein treatment affected ova-specific $\mathrm{CD} 8^{+} \mathrm{T}$ cell responses.

\subsection{Results}

\subsubsection{The decrease in $\mathrm{CD8}^{+} \mathrm{DC}$ is not due to the down regulation of $\mathrm{CD8}$ expression}

As described in Chapter 4, the number of splenic CD8 ${ }^{+}$DC was decreased 24-48 hr after treatment with $\alpha$-GalCer (Figure 4.2). Firstly, it was important to establish whether the $\mathrm{CD}^{+} \mathrm{DC}$ subset was decreased because of cell loss or a loss of the CD8 molecule on the DC surface. To assess this the Langerin-EGFP mice were utilised. Langerin-EGFP mice express enhanced green fluorescent protein (EGFP) under the control of the Langerin promoter (57). Langerin is expressed on splenic $\mathrm{CD}^{+} \mathrm{DC}$, whilst $\mathrm{CD} 8^{-} \mathrm{DC}$ have little to no expression (421-423). As reported in the literature (57) the CD11 $\mathrm{c}^{+} \mathrm{EGFP}^{+} \mathrm{DC}$ expressed CD8 and DEC 205, while CD11 $\mathrm{c}^{+}$EGFP $^{-}$DC had low expression of these markers (Figure 5.1 A and B). Furthermore, both CD11 ${ }^{+} \mathrm{EGFP}^{+} \mathrm{DC}$ and $\mathrm{CD} 8^{+} \mathrm{DC}$ from C57BL/6 mice expressed DEC 205 (Figure 5.1 B). To determine whether the $\mathrm{CD}^{+} \mathrm{DC}$ subset was decreased after $\alpha$-GalCer treatment the Langerin-EGFP mice were treated i.v. with $\alpha$-GalCer, then $24 \mathrm{hr}$ later the spleens were harvested and the number of $\mathrm{CD} 8^{+} \mathrm{DC}$ 
examined by flow cytometry. Langerin-EGFP mice treated with $\alpha$-GalCer showed a significant decrease in the number of $\mathrm{CD} 11 \mathrm{c}^{+} \mathrm{EGFP}^{+}$cells compared to the control mice (Figure 5.2). This indicated that the splenic $\mathrm{CD}^{+} \mathrm{DC}$ subset was lost after $\alpha$-GalCer treatment rather than down regulating the CD8 molecule.

A.

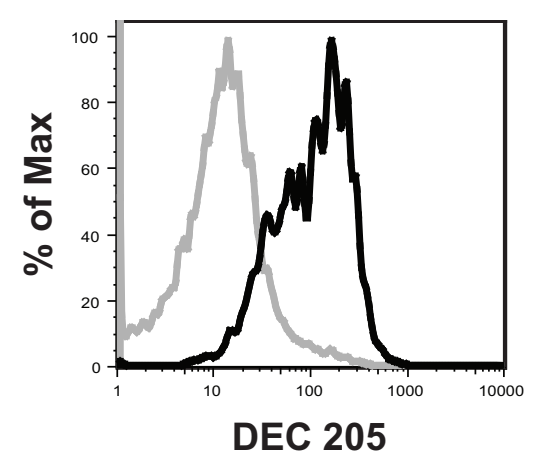

Key:

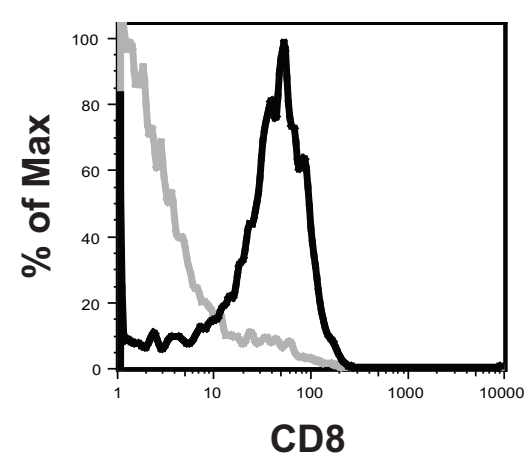

CD11 $\mathrm{c}^{+}$EGFP-

B.
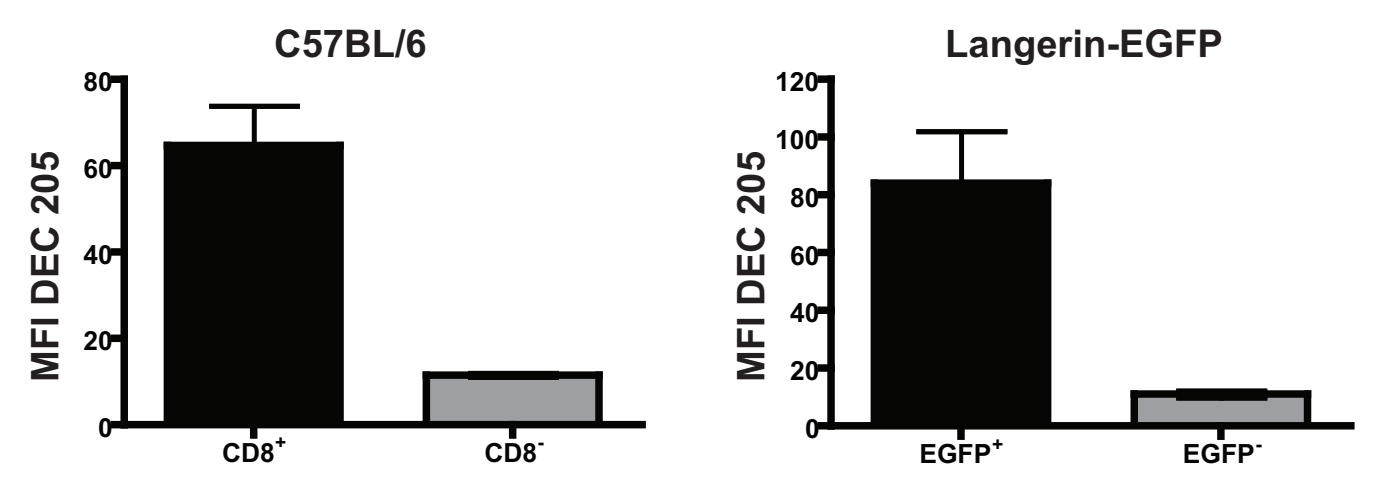

Figure 5.1 $\mathrm{EGFP}^{+}$splenic DC from the Langerin-EGFP mice are phenotypically similar to splenic $\mathrm{CD8}^{+}$DC from C57BL/6 mice.

Spleens were harvested from naïve C57BL/6 and Langerin-EGFP mice, digested and processed into single cell suspensions. The number of DC present was then determined by flow cytometry. Dead $\left(\mathrm{PI}^{+}\right)$and $\mathrm{B} 220^{+}$cells were excluded and lymphocytes were gated on the basis of their FSC vs. SSC properties. (A) Histograms show the expression of DEC 205 (left panel) and CD8 (right panel) from representative Langerin-EGFP mice. (B) Expression of DEC 205 from C57BL/6 (left panel) and Langerin-EFP mice (right panel). Bars represent mean \pm SD of 3 mice per group. 
Figure 5.2 The number of splenic DC expressing Langerin is reduced after treatment with $\alpha$ GalCer.

C57BL/6 and Langerin-EGFP mice were injected i.v. with PBS or $\alpha$-GalCer. Spleens were harvested $24 \mathrm{hr}$ later and analysed as described in Figure 5.1. (A) Dot plots show expression of EGFP on spleen cells from C57BL/6 and Langerin-EGFP mice treated with $\alpha$-GalCer or left untreated. The $\mathrm{EGFP}^{+}$population is indicated by an oval gate and the $\mathrm{EGFP}^{-}$population is indicated by a rectangular gate. (B) Total number of CD11 ${ }^{+}$EGFP $^{-}$cells (left panel) and

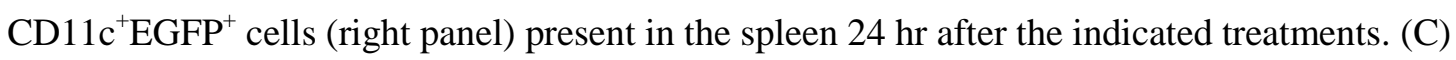
Expression of CD86 on CD11 ${ }^{+} \mathrm{EGFP}^{-}$cells (left panel) and CD11 $\mathrm{c}^{+} \mathrm{EGFP}^{+}$cells in the spleen 24 $\mathrm{hr}$ after the indicated treatment. Bars represent mean \pm SD or range of 2-3 mice per group. $* * *: p<$ 0.001 , and ns: not significant by an unpaired Student's $t$ test. These experiments were repeated with similar results. 
A.
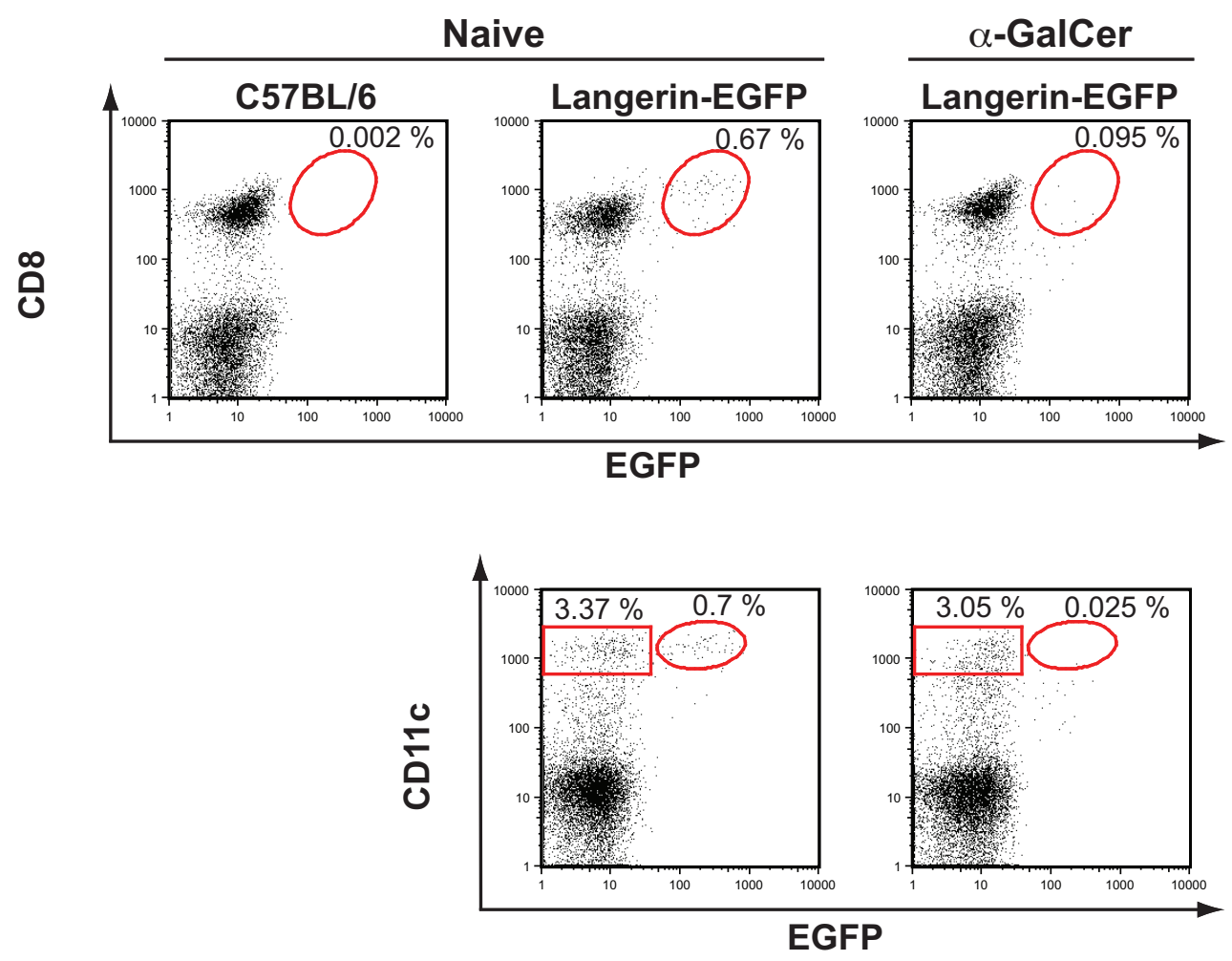

B.
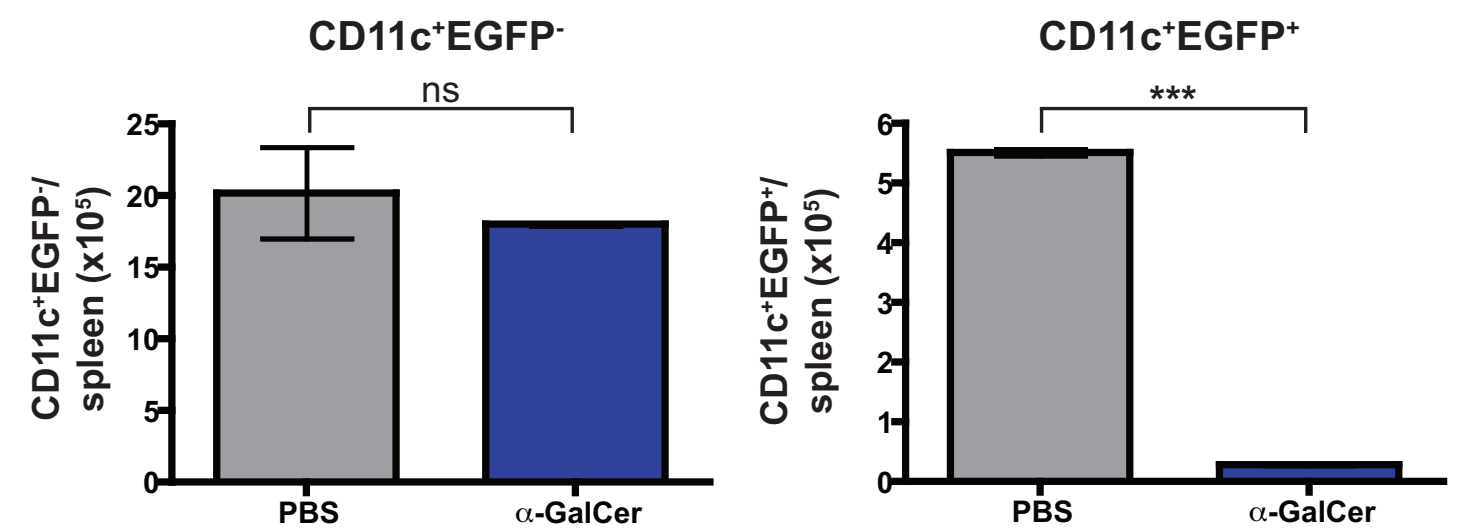

C.
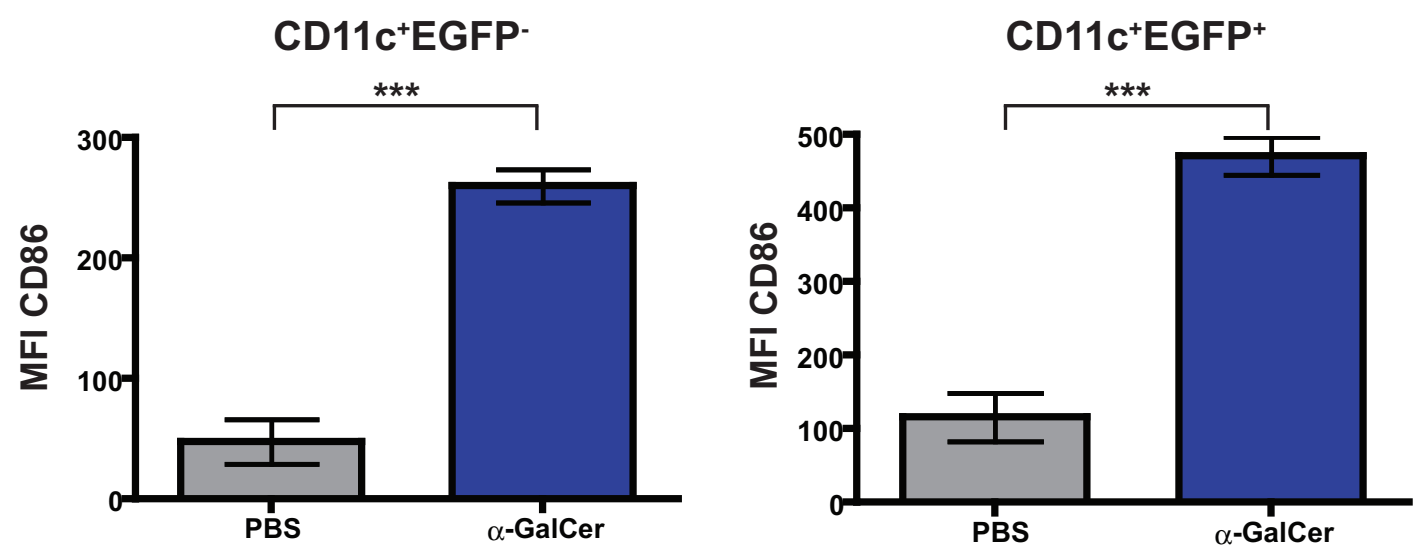
To obtain additional evidence on whether the $\mathrm{CD} 8^{+} \mathrm{DC}$ were lost after $\alpha$-GalCer treatment rather than downregulating expression of the CD8 molecule, the marker CD24 was also assessed, as splenic CD8 ${ }^{+}$DC express high levels of CD24 $(58,424)$. Indeed, $24 \mathrm{hr}$ after $\alpha-$ GalCer treatment the number of $\mathrm{CD} 11 \mathrm{c}^{+} \mathrm{CD} 24^{\mathrm{hi}} \mathrm{DC}$ was decreased in $\mathrm{C} 57 \mathrm{BL} / 6$ mice, while $\mathrm{CD} 11 \mathrm{c}^{+} \mathrm{CD} 24^{\text {lo }}$ cells remained stable (Figure 5.3). Together, these data strongly suggest that the $C D 8^{+}$DC subset in the spleen decreases after $\alpha$-GalCer treatment.
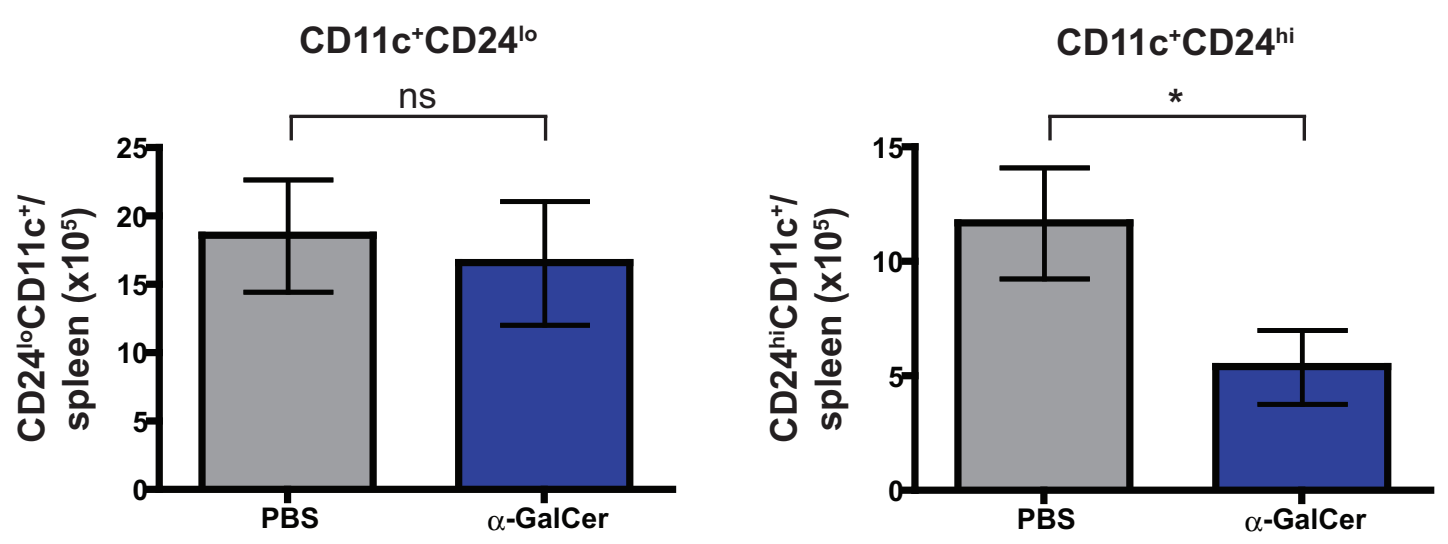

Figure 5.3 The number of ${\mathrm{CD} 11 \mathrm{c}^{+} \mathrm{CD24}}^{\text {hi }}$ cells declines after $\alpha$-GalCer treatment.

C57BL/6 mice were injected i.v. with either PBS or $\alpha$-GalCer. Spleens were harvested $24 \mathrm{hr}$ later and analysed as described in Figure 5.1. Total number of $\mathrm{CD} 11 \mathrm{c}^{+} \mathrm{CD} 24^{\text {lo }}$ cells (left panel) and $\mathrm{CD} 11 \mathrm{c}^{+} \mathrm{CD} 24^{\mathrm{hi}}$ cells (right panel) present in the spleen $24 \mathrm{hr}$ after the indicated treatments. Bars represent mean $\pm \mathrm{SD}$ or range of 2-3 mice per group. $*: p<0.05$, and ns: not significant by an unpaired Student's $t$ test.

There are additional cell surface markers that are routinely used to distinguish the $\mathrm{CD} 8^{+}$ DC from the CD8 ${ }^{-}$DC subset. DEC 205 is highly expressed on steady state CD8 ${ }^{+}$DC, but not on CD8 ${ }^{-}$DC (59). However, it was found that CD8 ${ }^{-}$DC upregulate expression of DEC 205 upon treatment with $\alpha$-GalCer (Figure 5.4 B). This is consistent with previously published data (319). Thus, DEC 205 was not a suitable marker to identify CD8 ${ }^{+}$DC after $\alpha$-GalCer treatment. 
$\mathrm{CD} 11 \mathrm{~b}$ is an additional cell surface marker that is highly expressed on CD8- DC (59). A marginal increase in the cell surface expression of CD11b was found on the CD8 ${ }^{+} \mathrm{DC}$ subset after $\alpha$-GalCer treatment (Figure 5.4 C). Increased expression of CD11b on the $\mathrm{CD}^{+} \mathrm{DC}$ has also been observed after overnight culture (59). Therefore it was decided not to use $\mathrm{CD} 1 \mathrm{~b}$ as an additional marker to establish whether the $\mathrm{CD} 8^{+} \mathrm{DC}$ subset was decreased. Altogether, the data from the Langerin-EGFP mice and the use of the cell surface marker $\mathrm{CD} 24$ provided convincing evidence that the splenic $\mathrm{CD} 8^{+} \mathrm{DC}$ subset was decreased after $\alpha$-GalCer treatment rather than downregulating the expression of CD8. 


\section{Figure 5.4 DEC 205 is a marker of steady state but not activated splenic $\mathrm{CD8}^{+}$DC.}

C57BL/6 mice were injected i.v. with either PBS or $\alpha$-GalCer. Spleens were harvested $24 \mathrm{hr}$ later and analysed as described in Figure 5.1. (A) Histograms show the expression of DEC 205 (left panel) and $\mathrm{CD} 11 \mathrm{~b}$ (right panel) on $\mathrm{CD}^{-} \mathrm{DC}$ and $\mathrm{CD} 8^{+} \mathrm{DC}$ from representative mice treated with PBS. (B) Dot plot show total CD11 $\mathrm{c}^{+}$cells and percentage of cells expressing DEC 205 and CD8 are indicated from representative mice treated with either PBS (left panel) or $\alpha$-GalCer (right panel). (C) Dot plot show total CD11 ${ }^{+}$cells and percentage of cells expressing CD11b and CD8 are indicated from representative mice treated with either PBS (left panel) or $\alpha$-GalCer (right panel). These experiments were repeated with similar results. 
A.
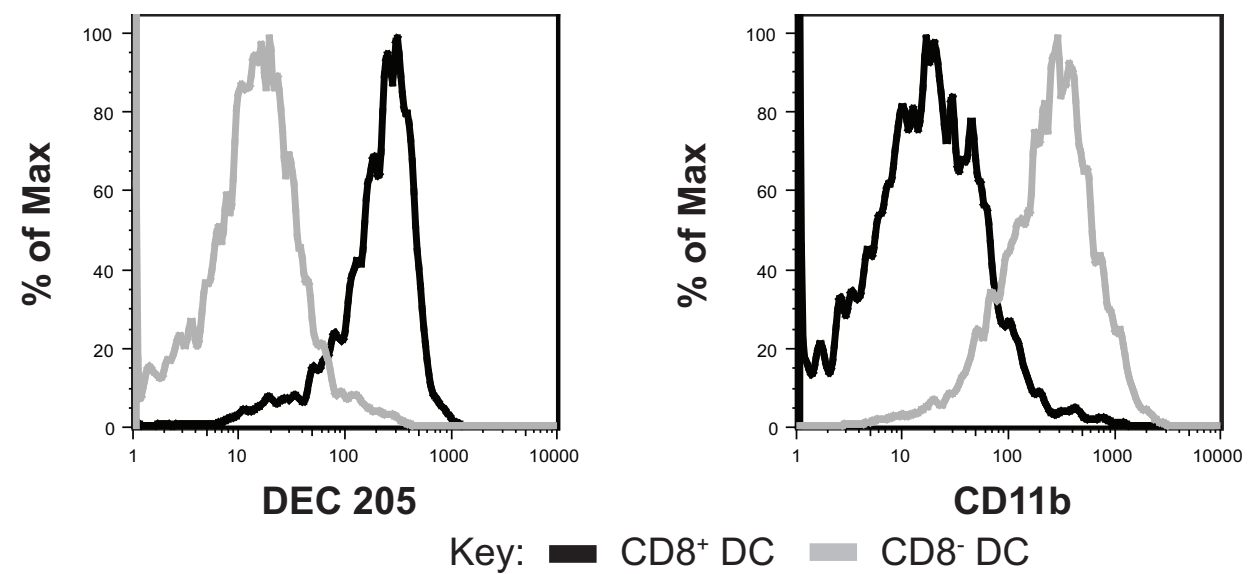

B.
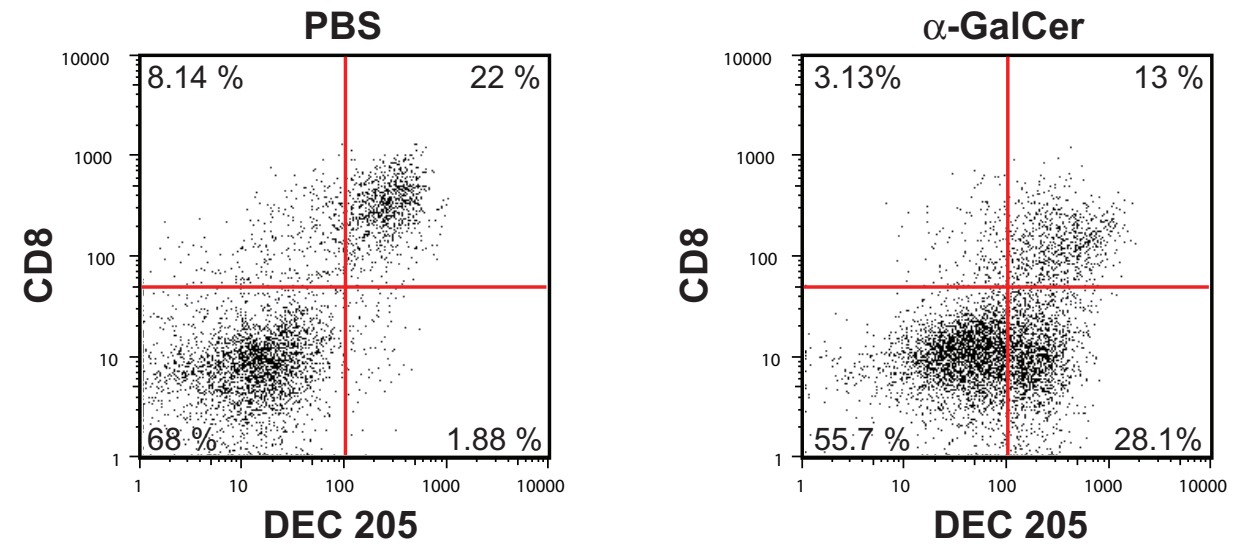

C.
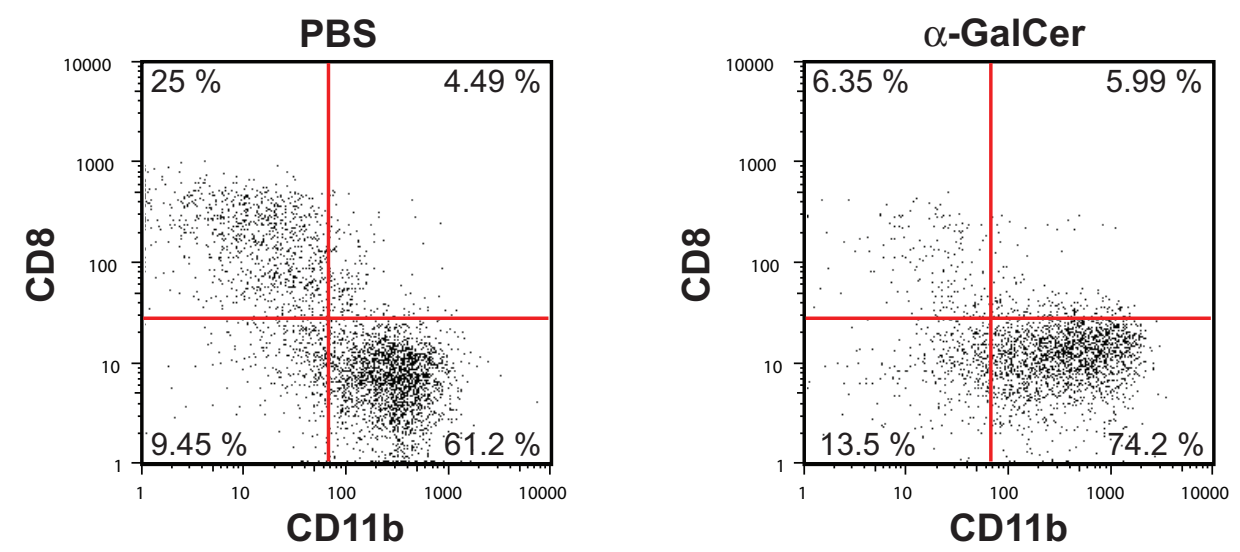


\subsubsection{The decrease in $\mathrm{CD8}^{+} \mathrm{DC}$ requires the presence of $\mathrm{CD1d}$ and/or NKT cells}

Since evidence presented in Figures 5.2 and 5.3 supports the possibility that $\mathrm{CD} 8^{+} \mathrm{DC}$ are lost after $\alpha$-GalCer treatment, it was of interest to determine the mechanism of this loss. To establish whether NKT cells were mediating CD8 ${ }^{+}$DC loss, it was next assessed whether the decrease in $\mathrm{CD}^{+} \mathrm{DC}$ was dependent on the presence of NKT cells. To evaluate this CD1 KO mice, which lack NKT cells, were treated with $\alpha$-GalCer and $24 \mathrm{hr}$ later the number of splenic $\mathrm{CD}^{+}$DC was examined by flow cytometry. As seen in Figure 5.5 A, no change in the number of either $\mathrm{CD}^{+}$or $\mathrm{CD} 8^{-} \mathrm{DC}$ was observed in $\mathrm{CD} 1 \mathrm{KO}$ mice. In addition, treatment of CD1 KO mice with $\alpha$-GalCer resulted in no increase in the expression of $\mathrm{CD} 86$ on either the $\mathrm{CD}^{+}$or $\mathrm{CD}^{-} \mathrm{DC}$ compared to the PBS control group (Figure 5.5 B). This was in contrast to C57BL/6 mice where an increase in the expression of CD86 was seen after $\alpha$-GalCer treatment (Figure 4.3 B). These data illustrate that the decline in $\mathrm{CD}^{+}$DC required the presence of NKT cells, which is a novel finding, and it confirmed, as reported by others $(319,320,425)$ that CD1d is necessary for the activation of DC after $\alpha$-GalCer treatment. 
A.

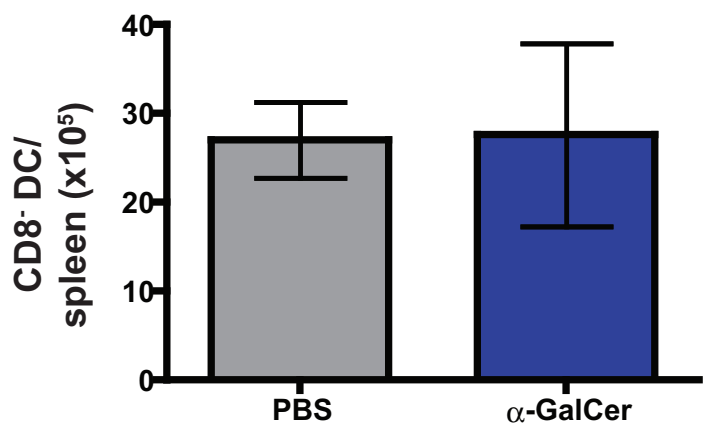

B.

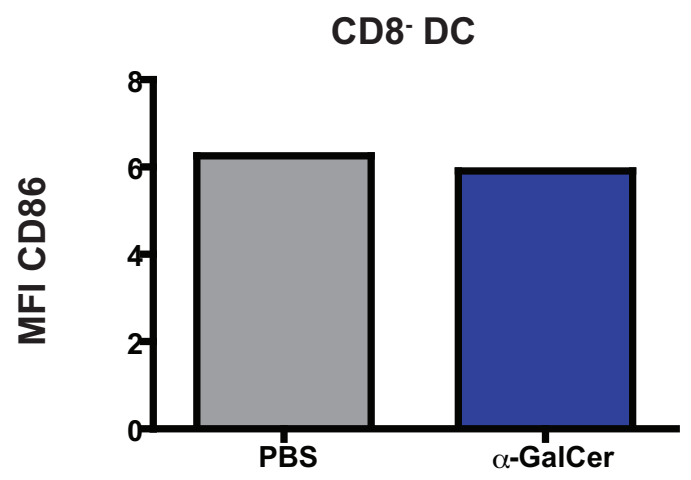

CD8 + DC
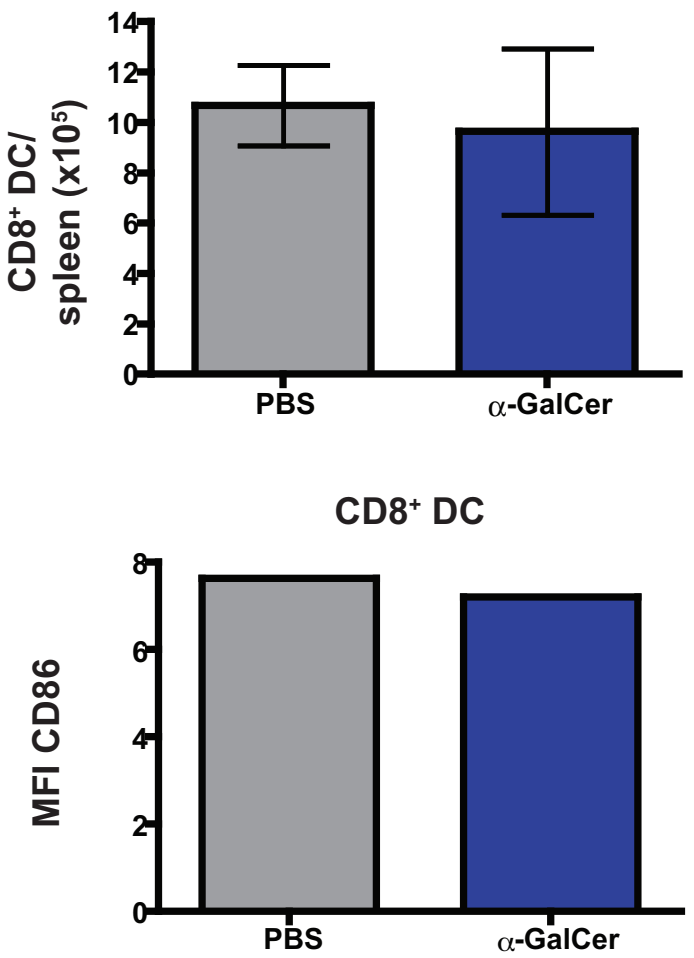

Figure 5.5 The decrease in $\mathrm{CD8}^{+} \mathrm{DC}$ after $\alpha$-GalCer treatment requires NKT cells.

CD1 KO mice were injected i.v. with PBS or $\alpha$-GalCer. Spleens were harvested $24 \mathrm{hr}$ later and analysed as described in Figure 5.1. (A) Total number of CD8 ${ }^{-}$DC (left panel) and CD8 ${ }^{+}$DC (right panel) present in the spleen $24 \mathrm{hr}$ after the indicated treatment. Bars represent mean \pm SD of 4 mice per group. (B) Expression of CD86 on the CD8 ${ }^{-}$DC (left panel) and CD8 ${ }^{+}$DC (right panel) in the spleen $24 \mathrm{hr}$ after the indicated treatment. Bars indicate CD86 MFI in individual mice. This experiment was repeated with similar results. 


\subsubsection{The reduction in $\mathrm{CD8}^{+} \mathrm{DC}$ after $\alpha$-GalCer treatment occurs in a dose dependent manner}

To further characterise the effect of $\alpha$-GalCer, the dose required to induce loss of CD $8^{+}$ DC was determined. Administration of $1 \mathrm{ng}$ of $\alpha$-GalCer caused no significant decrease in the number of $\mathrm{CD}^{+} \mathrm{DC}$, while an intermediate dose $(8 \mathrm{ng})$ resulted in a significant decline but not as dramatic as the high dose (200 ng) of $\alpha$-GalCer (Figure 5.6 A). None of these doses of $\alpha$-GalCer affected the number of splenic CD8 ${ }^{-}$DC. However, at low doses of $\alpha$ GalCer, the expression of CD86 on $\mathrm{CD}^{+}$and $\mathrm{CD}^{-} \mathrm{DC}$ was lower than in mice treated with $200 \mathrm{ng} \alpha$-GalCer (Figure 5.6 B). The decrease in $\mathrm{CD}^{+} \mathrm{DC}$ numbers and the increased cell surface expression of CD86 were both observed at the same dose of $\alpha$ GalCer (Figure 5.6), suggesting that DC activation and loss of the $\mathrm{CD} 8^{+} \mathrm{DC}$ subset may be triggered in the same conditions, and might be related events. 
A.

CD8- DC

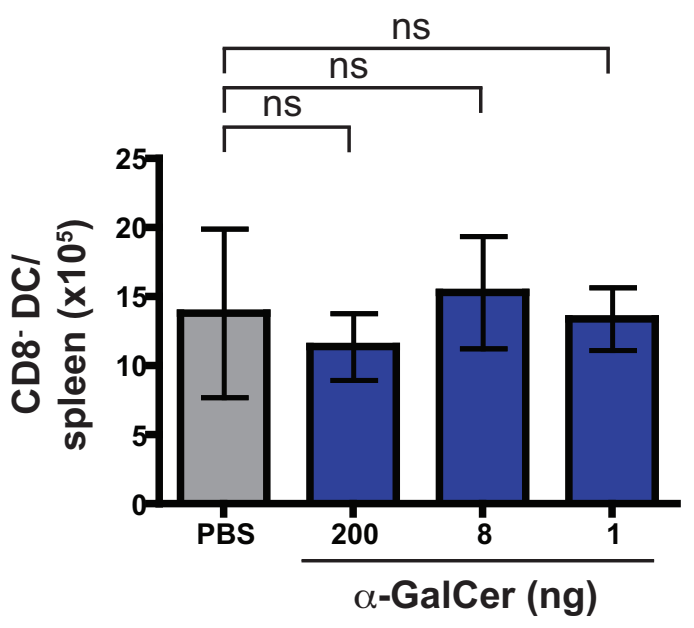

B.

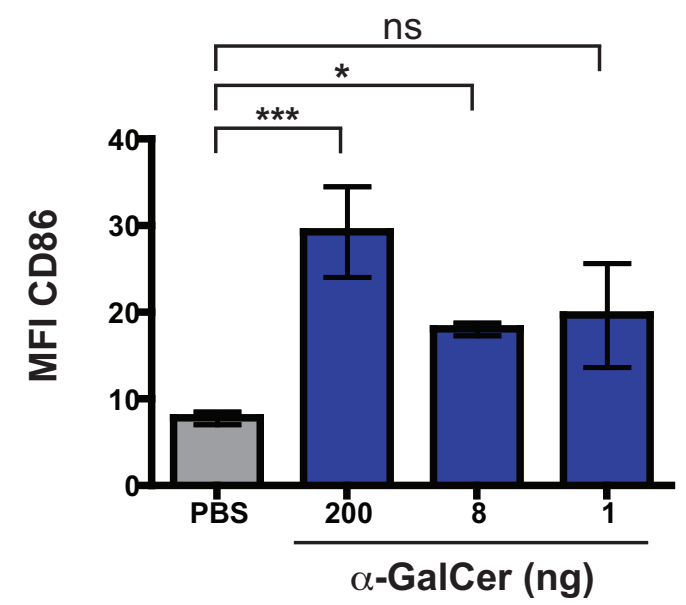

CD8+ DC
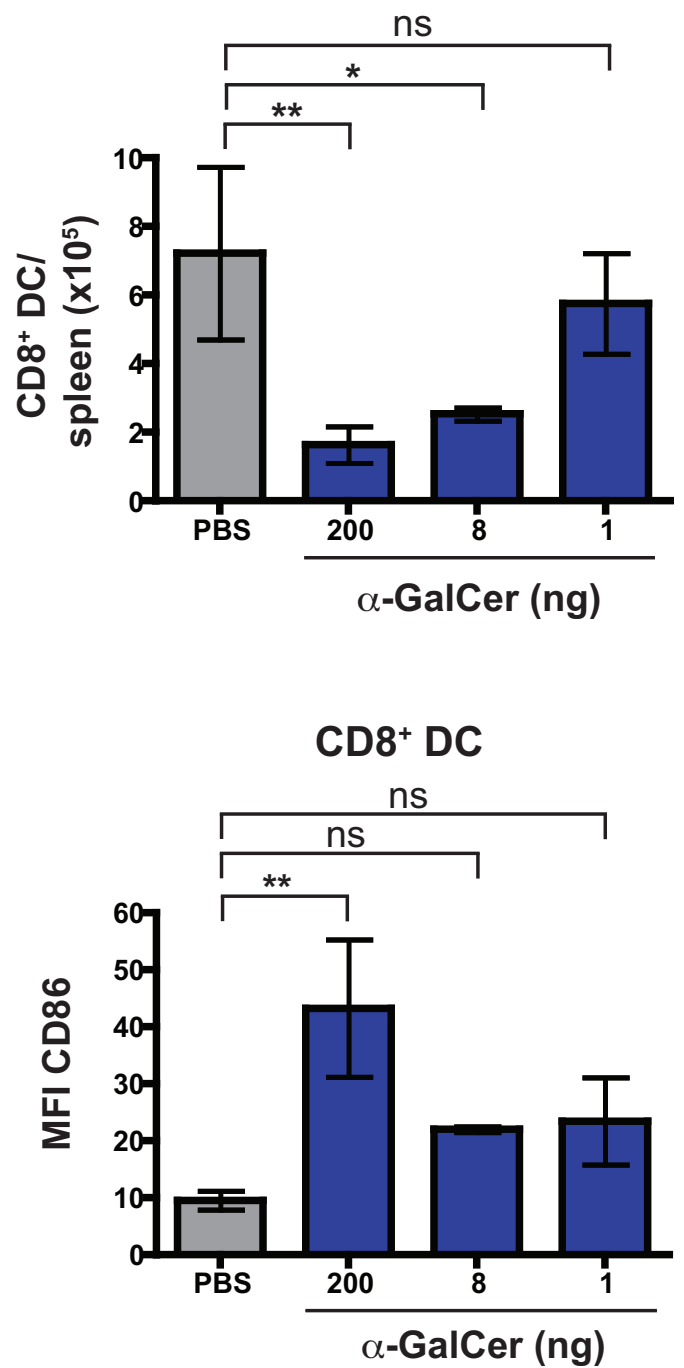

Figure 5.6 The decrease in the number of $\mathrm{CD8}^{+} \mathrm{DC}$ occurs in a dose dependent manner.

C57BL/6 mice were injected i.v. with PBS or the indicated amount of $\alpha$-GalCer. Spleens were harvested $24 \mathrm{hr}$ later and analysed as described in Figure 5.1. (A) Total number of CD8- DC (left panel) and CD8 ${ }^{+} \mathrm{DC}$ (right panel) present in the spleen $24 \mathrm{hr}$ after the indicated dose of $\alpha$-GalCer or PBS. (B) Expression of CD86 on the CD8 ${ }^{-}$DC (left panel) and CD8 ${ }^{+}$DC (right panel) in the spleen $24 \mathrm{hr}$ after the administration of $\alpha-G a l C e r$ or PBS. Bars represent mean \pm SD of 3 mice per group. $* * *: p<0.001, * *: 0.001<p<0.01, *: p<0.05$, and ns: not significant by one-way ANOVA with a Tukey post test. This experiment was repeated with similar results. 


\subsubsection{The decline in $\mathrm{CD8}^{+} \mathrm{DC}$ after $\alpha$-GalCer treatment is transient}

The results presented so far demonstrated that the decrease in $\mathrm{CD}^{+} \mathrm{DC} 24 \mathrm{hr}$ after $\alpha$ GalCer treatment required NKT cells and occurred in a dose dependent manner. Next it was sought to characterise the kinetics of the decrease in the $\mathrm{CD} 8^{+} \mathrm{DC}$ subset. A time course of the loss of $\mathrm{CD}^{+} \mathrm{DC}$ was determined by treating mice with $\alpha$-GalCer and at specific time points after treatment harvesting the spleens and determining the number of DC by flow cytometry. There was a modest but not statistically significant decrease in the number of $\mathrm{CD}^{+}$DC $10 \mathrm{hr}$ after $\alpha$-GalCer treatment (Figure 5.7 A), however, a significant decline in $\mathrm{CD}^{+} \mathrm{DC}$ was apparent as early as $15 \mathrm{hr}$ after $\alpha$-GalCer treatment with the greatest reduction occurring $24 \mathrm{hr}$ after treatment (Figure 5.7 B and C). Seven days after $\alpha$ GalCer treatment the number of $\mathrm{CD}^{+} \mathrm{DC}$ was similar to the control group (Figure 5.7 B). After $\alpha$-GalCer treatment the activation status of the DC was assessed by investigating the cell surface expression of CD86 (Figure 5.7 D). Similar to what was observed previously in Figure 5.6 B, the expression of CD86 was increased in both $\mathrm{CD}^{+}$and CD8- DC subsets after $\alpha$-GalCer treatment (Figure 5.7 D). These results indicate that the loss of $\mathrm{CD}^{+} \mathrm{DC}$ occurred early after $\alpha$-GalCer treatment but that this loss was only transient. 


\section{Figure 5.7 The loss of $\mathrm{CD8}^{+} \mathrm{DC}$ after $\alpha$-GalCer treatment is transient.}

C57BL/6 mice were injected i.v. with either PBS or $200 \mathrm{ng} \alpha$-GalCer. At 10 hours (A) or the indicated time point after treatment the spleens were harvested and analysed as described in Figure 5.1. (A-C) Total numbers of $\mathrm{CD} 8^{+} \mathrm{DC}$ present in the spleen at the indicated times after treatment with $\alpha$-GalCer. (D) Expression of CD86 on CD8 ${ }^{-}$DC (left panel) and CD8 ${ }^{+}$DC (right panel) in the spleen at the indicated time points after treatment with $\alpha$-GalCer. Bars represent mean \pm SD of 3-4 mice per group. (A) ns: not significant by an unpaired Student's $t$ test. (B-D) $* * *: p<0.001$, **: $0.001<p<0.01$, and $*: p<0.05$ by one-way ANOVA with a Tukey post test. These experiments were repeated with a similar result. 
A.

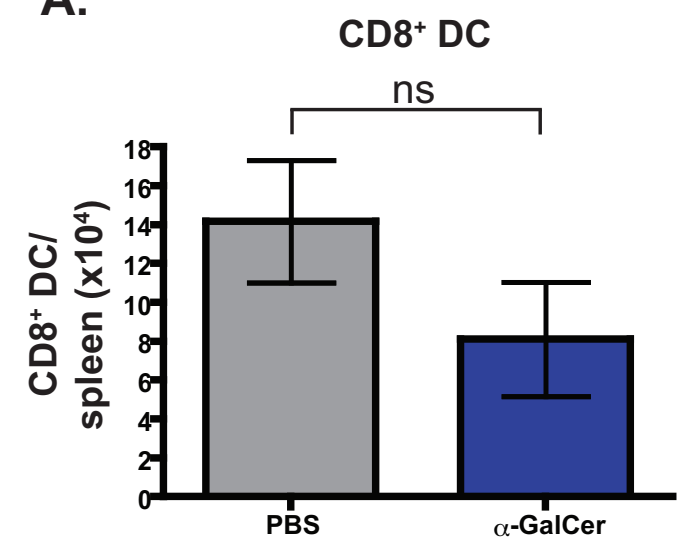

B.
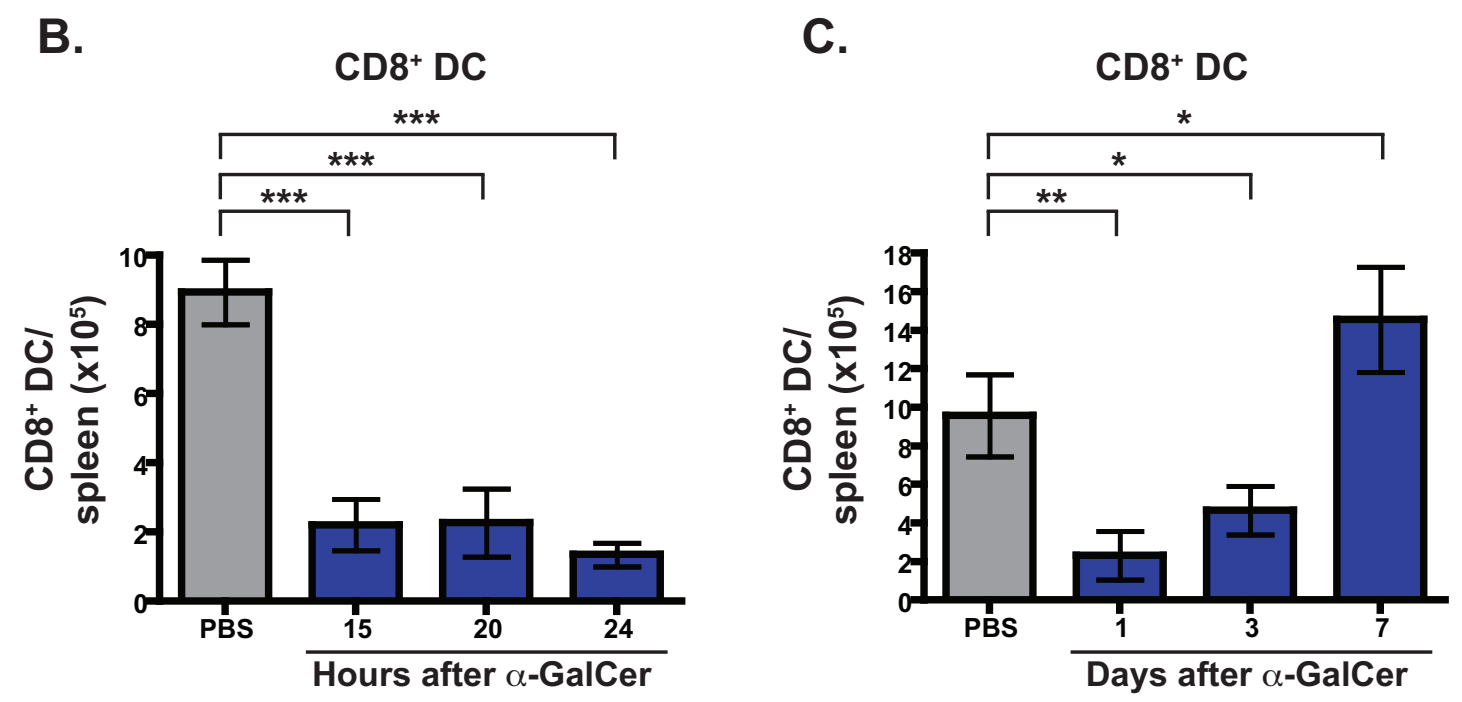

D.
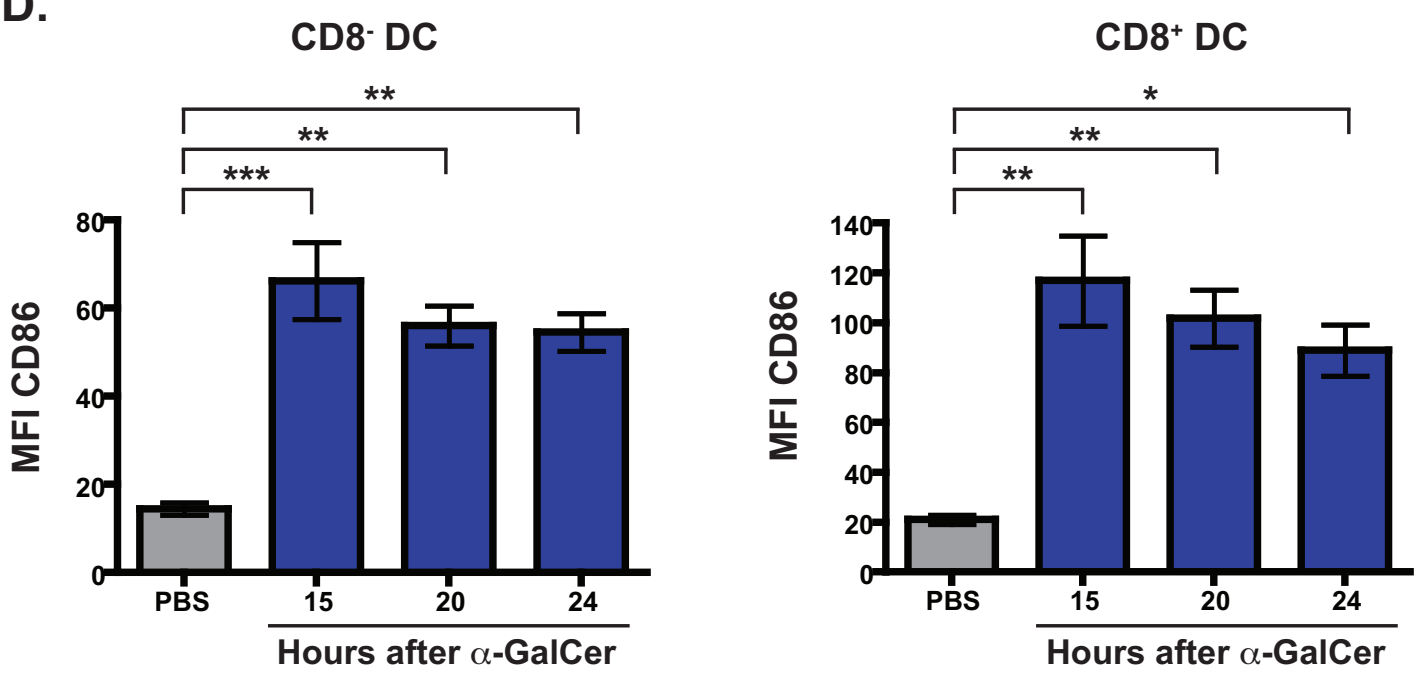


\subsection{5 $\mathrm{CD8}^{+} \mathrm{DC}$ express higher levels of CD1d}

These data presented so far show that the $\mathrm{CD} 8^{+} \mathrm{DC}$ subset was preferentially decreased by $\alpha$-GalCer treatment. It is not clear why only the $\mathrm{CD} 8^{+} \mathrm{DC}$ subset was lost after $\alpha$-GalCer treatment as both DC subsets were activated by the treatment. One possible explanation is that the $\mathrm{CD}^{+} \mathrm{DC}$ express a higher amount of CD1d on their cell surface, which might promote greater interaction with NKT cells. Expression of CD1d was examined by flow cytometry. Steady state $\mathrm{CD}^{+} \mathrm{DC}$ were found to express 2-fold higher levels of CD1d compared to $\mathrm{CD}^{-} \mathrm{DC}$ (Figure 5.8). A similar result was also reported by others using a global gene analysis of the DC subsets using Affymetrix microarrays (64). Thus it is possible that the $\mathrm{CD} 8^{+} \mathrm{DC}$ are preferentially involved in the presentation of glycolipids to NKT cells.

A.

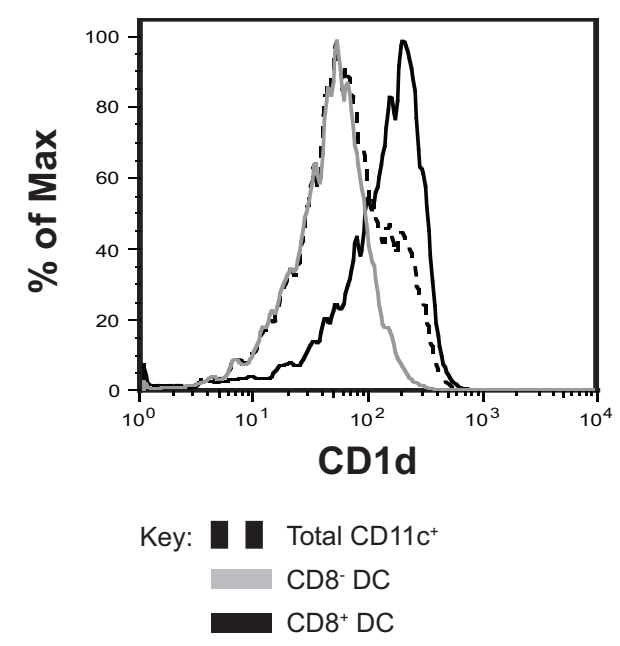

B.

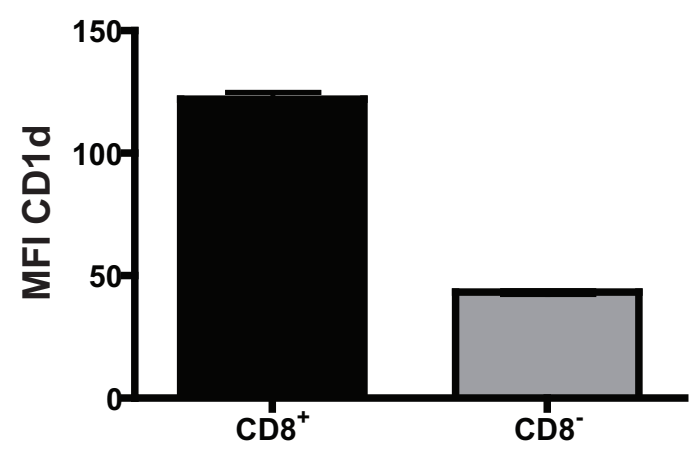

Figure 5.8 $\mathrm{CD8}^{+}$DC express higher levels of surface CD1d than $\mathrm{CD8}^{-} \mathrm{DC}$.

Spleen DC were obtained from untreated C57BL/6 mice. (A) Histograms show the expression of CD1d on total CD11 ${ }^{+}$cells (dashed line), CD8 ${ }^{-}$DC (grey line) and $\mathrm{CD}^{+}$DC (black line) from the spleen of untreated C57BL/6 mice. (B) Expression of CD1d on CD8 ${ }^{+}$DC and CD8- DC. Bars represent mean \pm range of 2 mice per group. This experiment was repeated with a similar result. 


\subsubsection{Treatment with the $\alpha$-GalCer analogue $\mathrm{OCH}$ induces a decrease in the number of spleen $\mathrm{CD8}^{+} \mathrm{DC}$}

A number of synthetic analogues of $\alpha-G a l C e r$ have been produced, which induce distinct functional responses from NKT cells. The analogue OCH has a substantially shorter sphingosine chain compared to $\alpha-G a l C e r$, and when injected into mice it induces the preferential secretion of IL-4 (305-307) with kinetics similar to $\alpha$-GalCer $(305,307)$. In addition, $\mathrm{OCH}$ has a reduced binding affinity to $\mathrm{CD} 1 \mathrm{~d}$ compared to $\alpha$-GalCer $(306,426)$ and is less potent at activating NKT cells $(305,307)$. Thus it was of interest to determine whether treatment with $\mathrm{OCH}$ also resulted in a decrease in the splenic $\mathrm{CD} 8^{+} \mathrm{DC}$. Mice were treated i.v. with $\mathrm{OCH}$ and $24 \mathrm{hr}$ later the number of splenic $\mathrm{CD} 8^{+} \mathrm{DC}$ was assessed. As shown in Figure 5.9 A, after treatment of C57BL/6 mice with $\mathrm{OCH}$ the number of $\mathrm{CD}^{+} \mathrm{DC}$ was significantly decreased. Comparable doses of $\alpha-\mathrm{GalCer}$ and $\mathrm{OCH}$ were required for this effect (Figure 5.9). Similar results were also observed in PKO mice and no significant change in the number of $\mathrm{CD}^{-}{ }^{-} \mathrm{DC}$ was observed in either C57BL/6 or PKO mice (Figure 5.9 A). A titration of $\mathrm{OCH}$ was carried out and the decrease in $\mathrm{CD}^{+} \mathrm{DC}$ was found to occur in a dose dependent manner (Figure 5.9 B) and require doses similar to $\alpha$ GalCer treatment (Figure 5.6 A). These data would indicate that in this mouse system the loss of $\mathrm{CD}^{+} \mathrm{DC}$ was not affected by the different NKT cell ligands and their different binding affinity for CD1d. 
A.

CD8- DC

CD8 + DC
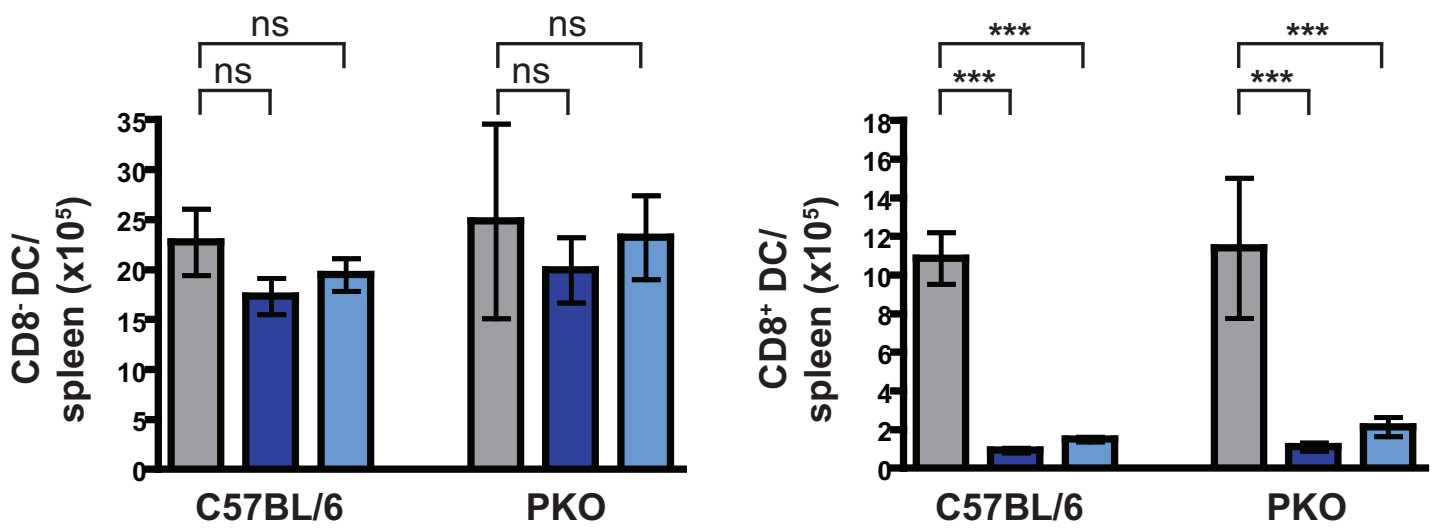

Key:

PBS

$\alpha-$ GalCer (200 ng)

$\square \mathrm{OCH}(200 \mathrm{ng})$

B.
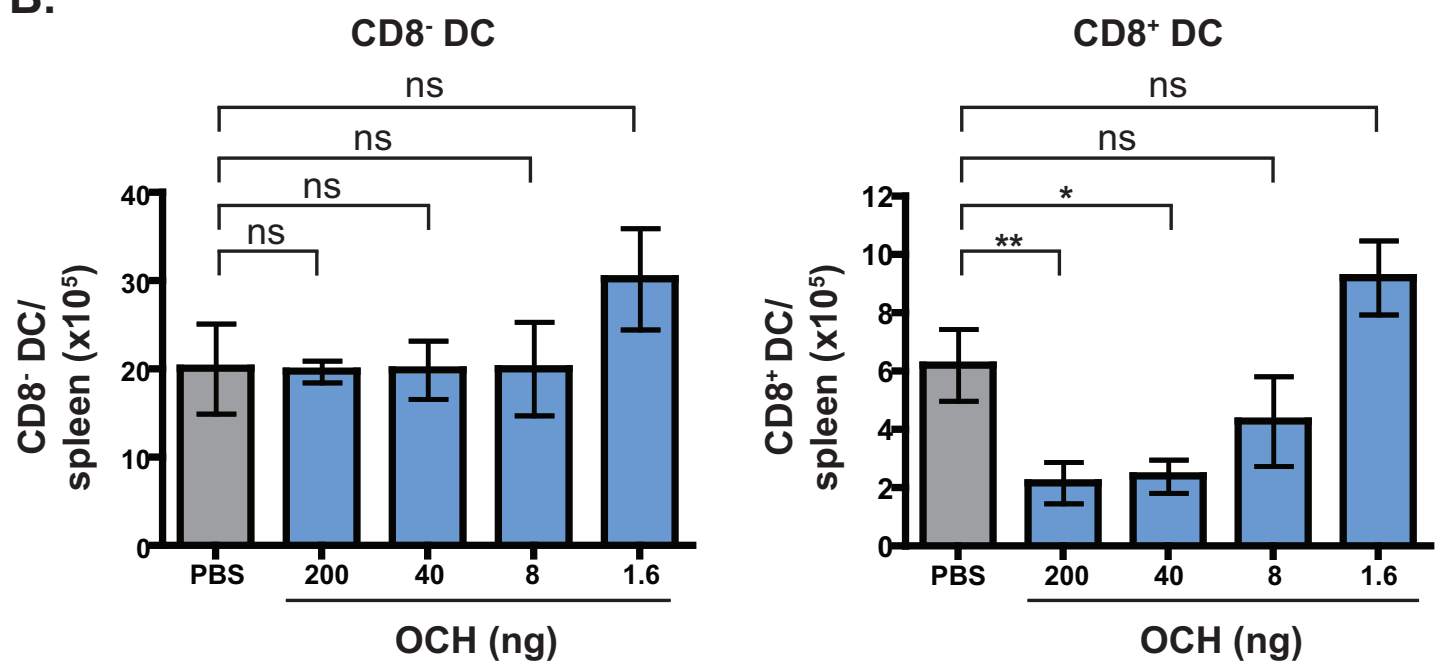

Figure 5.9 Treatment with the $\alpha$-GalCer analogue $\mathrm{OCH}$ induces a decline in the number of CD8 $^{+}$DC.

C57BL/6 and PKO mice were injected i.v. with either PBS, $\alpha$-GalCer or OCH as indicated.

Spleens were harvested $48 \mathrm{hr}$ later and analysed as described in Figure 5.1. (A) Total number of CD8 ${ }^{-}$DC (left panel) and CD8 ${ }^{+}$DC (right panel) present in the spleen after the indicated treatments. (B) Total number CD8 ${ }^{-}$DC (left panel) and CD8 ${ }^{+}$DC (right panel) present in the spleen after treatment with the indicated dose of $\mathrm{OCH}$. Bars represent mean \pm SD of 3-5 mice per group. $* * *: p<0.001, * *: 0.001<p<0.01, *: p<0.05$ and ns: not significant by one-way ANOVA with a Tukey post test. These experiments were repeated with similar results. 


\subsubsection{The decline in $\mathrm{CD8}^{+} \mathrm{DC}$ may not be mediated through a direct interaction with NKT cells}

To determine whether a direct cell-cell contact mechanism was required for the decline in $\mathrm{CD}^{+} \mathrm{DC}$ after $\alpha$-GalCer treatment, an adoptive transfer method was used. BMDC were loaded with $\alpha$-GalCer in vitro and adoptively transferred into naïve mice. Since it was assumed that in this experimental set up only the adoptively transferred BMDC were presenting $\alpha$-GalCer on their cell surface, no direct interaction would be required between NKT cells and $\mathrm{CD}^{+} \mathrm{DC}$.

The number of resident splenic $\mathrm{CD} 8^{+} \mathrm{DC}$ was similarly reduced in mice treated with BMDC loaded with $\alpha$-GalCer and mice treated i.v. with $\alpha$-GalCer (Figure 5.10 A), suggesting that the loss of $\mathrm{CD} 8^{+} \mathrm{DC}$ was not mediated by a direct interaction with the NKT cells. However, when CD1 KO BMDC, which are able to take up $\alpha$-GalCer but not present it on their cell surface, were loaded with $\alpha$-GalCer and injected into mice, a decrease in the splenic $\mathrm{CD} 8^{+} \mathrm{DC}$ was also observed (Figure 5.10 B). This result suggested that host cells were taking up the adoptively transferred CD1 KO BMDC and presenting the $\alpha$-GalCer on their cell surface. Again it was possible that this host population was the $\mathrm{CD}^{+} \mathrm{DC}$, as these cells are known to be able to efficiently take up and cross present soluble antigen and apoptotic cells in blood.

The activation status of the host splenic DC was also assessed by determining the expression level of CD86 (Figure 5.10 C and D). Interestingly, DC from mice that received either C57BL/6 or CD1 KO BMDC loaded with $\alpha$-GalCer expressed lower CD86 compared to DC from mice treated i.v. with $\alpha$-GalCer, but greater than mice that had received unloaded BMDC (Figure 5.10 C and D). Since CD86 expression on DC from mice injected with either C57BL/6 and CD1 KO BMDC loaded with $\alpha$-GalCer was similar, it is likely that the same mechanism was leading to DC activation. Since the number of splenic $\mathrm{CD}^{+} \mathrm{DC}$ was reduced after adoptive transfer of either C57BL/6 or CD1 KO BMDC, no conclusion could be made as to whether a direct interaction between the NKT cell and DC was required for the decrease in splenic $\mathrm{CD} 8^{+} \mathrm{DC}$. 


\section{Figure 5.10 The decrease in the number of $\mathrm{CD8}^{+} \mathrm{DC}$ may not require a direct interaction} with NKT cells.

C57BL/6 mice were injected i.v. with 3 x $10^{6} \mathrm{BMDC}$ from either C57BL/6 (A, B, left panel and C) or CD1 KO (B, right panel and C) mice, which were untreated or loaded with $\alpha$-GalCer. As a positive control, mice were treated with $\alpha$-GalCer alone. Spleens were harvested $24 \mathrm{hr}$ later and analysed as described in Figure 5.1. (A) Total number of CD8 ${ }^{-}$DC (left panel) and CD8 ${ }^{+}$DC (right panel) present in the spleen after the indicated treatments. (B) Total number of $\mathrm{CD} 8^{+} \mathrm{DC}$ in the spleen $24 \mathrm{hr}$ after adoptive transfer of either C57BL/6 BMDC (left panel) or CD1 KO BMDC (right panel). (C) Representative histograms from 1 out of 3 mice show the expression of CD86 on $\mathrm{CD}^{+}$DC $24 \mathrm{hr}$ after the indicated treatment. (D) The expression of CD86 on CD8 ${ }^{+}$DC $24 \mathrm{hr}$ after the indicated treatment. Bars represent mean \pm SD or range of 2-3 mice per group. $* * *: p<0.001$ and ns: not significant by one-way ANOVA with a Tukey post test. These experiments were repeated with similar results. 
A.
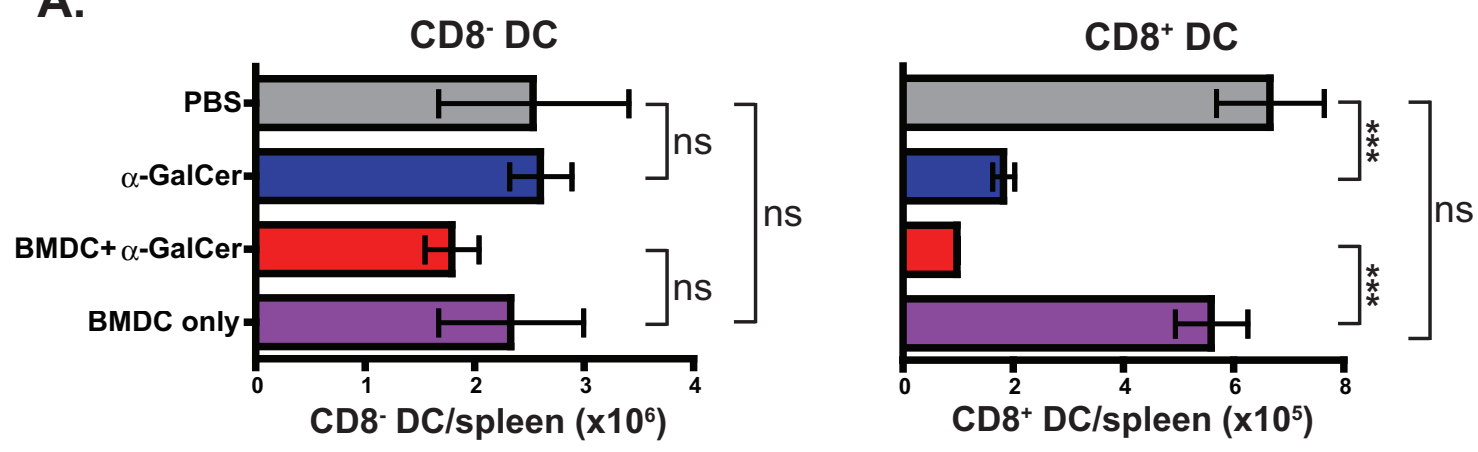

B.
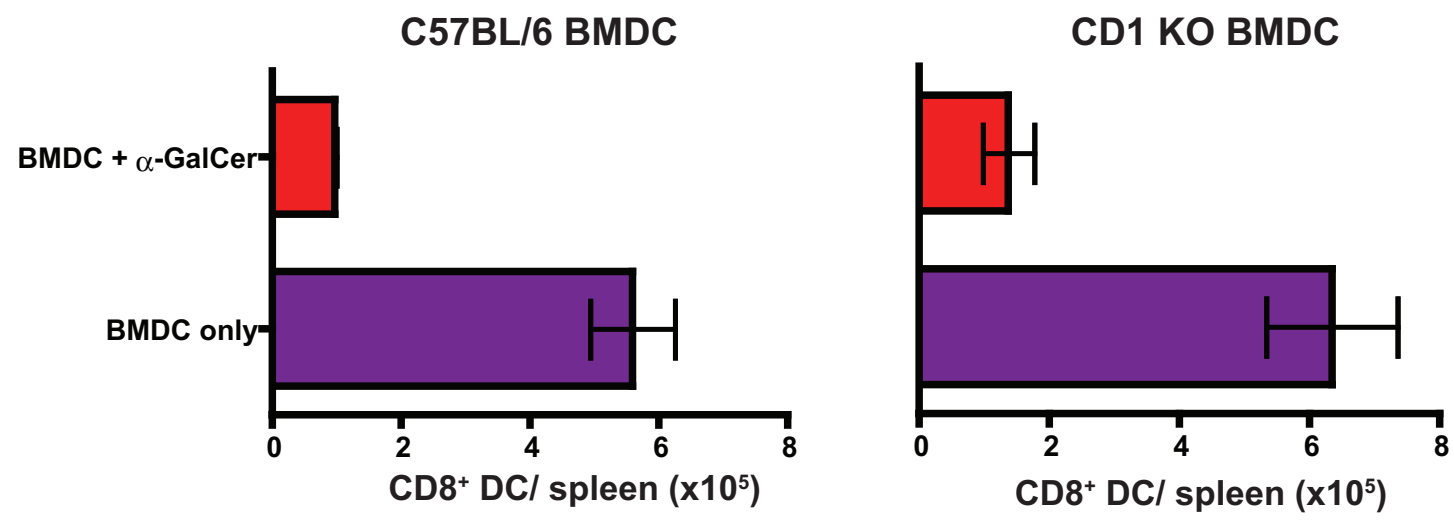

C.

D.

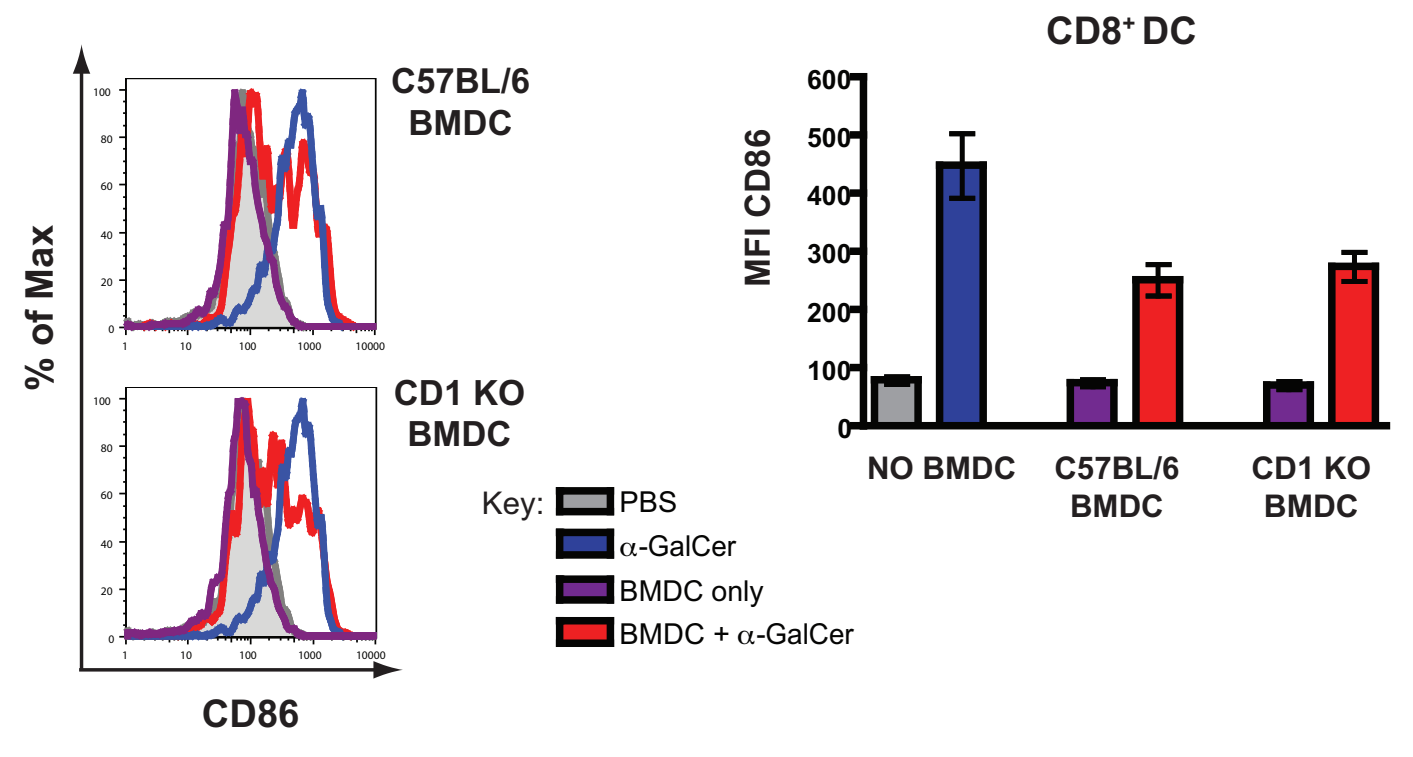




\subsubsection{IFN- $\gamma$ does not mediate the decrease in $\mathrm{CD8}^{+} \mathrm{DC}$ after treatment with $\alpha$-GalCer}

Adoptive transfer of BMDC did not provide direct evidence as to whether the decrease in $\mathrm{CD}^{+} \mathrm{DC}$ required a direct cell-cell contact between NKT cells and the $\mathrm{CD} 8^{+} \mathrm{DC}$ (Figure 5.10). Additionally, as described in Chapter 4, neither perforin nor FasL are required for the observed decrease in $\mathrm{CD}^{+} \mathrm{DC}$, further supporting the possibility that the decrease in $\mathrm{CD}^{+} \mathrm{DC}$ does not require a cell contact dependent mechanism (Figure 4.3 A and 4.6 A). Therefore the loss of $\mathrm{CD}^{+} \mathrm{DC}$ after treatment with NKT cell ligands could be cytokine dependent. A possible candidate was IFN- $\gamma$, which is rapidly produced by NKT cells after treatment of $\alpha$-GalCer. To determine if IFN- $\gamma$ played a role in the decrease of $\mathrm{CD}^{+} \mathrm{DC}$, mice lacking the ability to produce IFN- $\gamma(\mathrm{IFN}-\gamma \mathrm{KO})$ were utilised. As in C57BL/6 mice, a significant decrease in the number of $\mathrm{CD}^{+} \mathrm{DC}$ was observed in IFN- $\gamma$ KO mice $24 \mathrm{hr}$ after $\alpha$-GalCer treatment. Furthermore, no significant change in the number of CD8 ${ }^{-}$DC was observed in the IFN- $\gamma$ KO mice (Figure 5.11 A). Interestingly, DC from $\alpha$-GalCer treated IFN- $\gamma$ KO mice did not upregulate the expression of CD86 as profoundly as DC in C57BL/6 mice (Figure 5.11 C). This result is consistent with previously published work reporting that IFN- $\gamma$ was required for the increase in CD86 after $\alpha$-GalCer treatment (187). In addition, CD86 upregulation was especially weak on the CD8- DC subset suggesting that $\mathrm{CD}^{+}$and $\mathrm{CD}^{-}$DC subsets may respond differentially to $\alpha$-GalCer dependent stimulation. These data indicate that IFN- $\gamma$ does not mediate the loss of CD $8^{+}$DC after $\alpha-$ GalCer treatment. 
Figure 5.11 IFN- $\gamma$ does not mediate the loss of $\mathrm{CD8}^{+} \mathrm{DC}$ after $\alpha$-GalCer treatment.

C57BL/6 and IFN- $\gamma$ KO mice were injected i.v. with PBS or $\alpha$-GalCer. Spleens were harvested 24 hr later and analysed as described in Figure 5.1. (A) Dot plots show flow cytometric analysis of spleen cells, CD8 ${ }^{-} \mathrm{DC}$ are indicated by a rectangle gate, and $\mathrm{CD} 8^{+} \mathrm{DC}$ are indicated by an oval gate. (B) Total number of CD8 ${ }^{-}$DC (left panel) and CD8 ${ }^{+}$DC (right panel) present in the spleen 24 hr after the indicated treatment. (C) Expression of CD86 on the CD8 ${ }^{-}$DC (left panel) and CD8 ${ }^{+}$DC (right panel) in the spleen $24 \mathrm{hr}$ after the indicated treatment. Bars represent mean $\pm \mathrm{SD}$ of 3 mice per group. $* * *: p<0.001, * *: 0.001<p<0.01, *: p<0.05$, and ns: not significant by one-way ANOVA with a Tukey post test. These experiments were repeated three times with similar results. 
A.

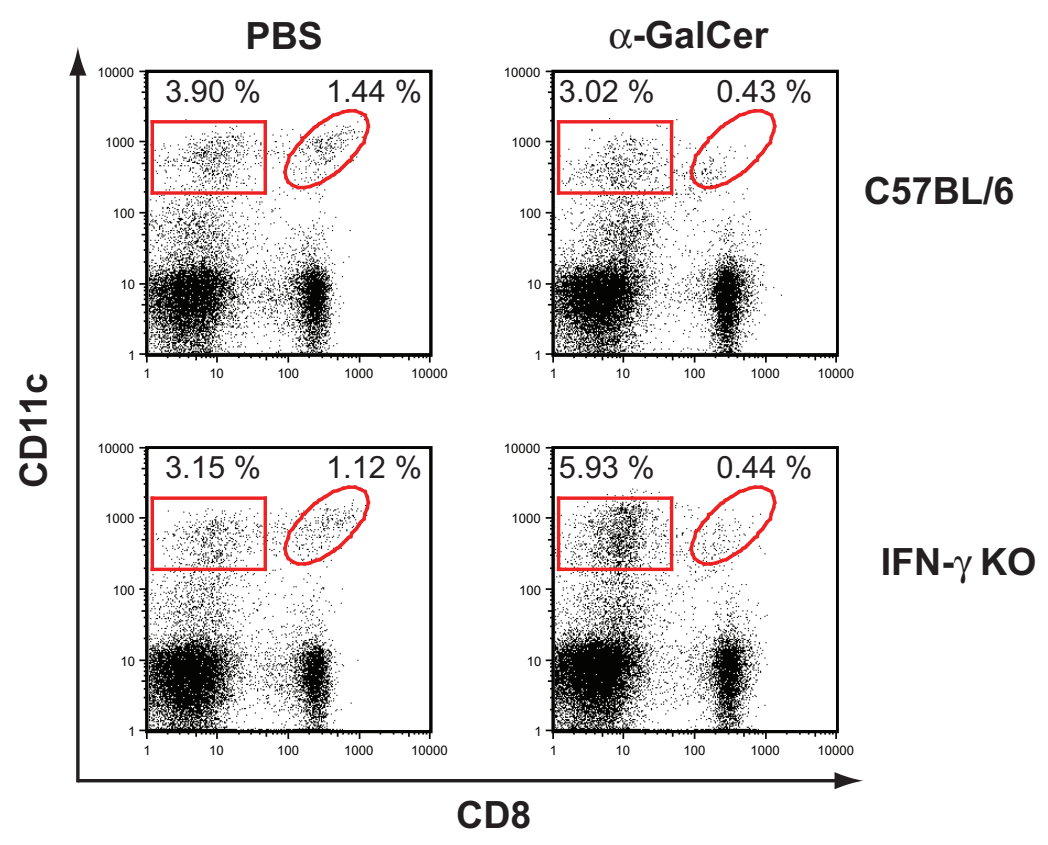

B.
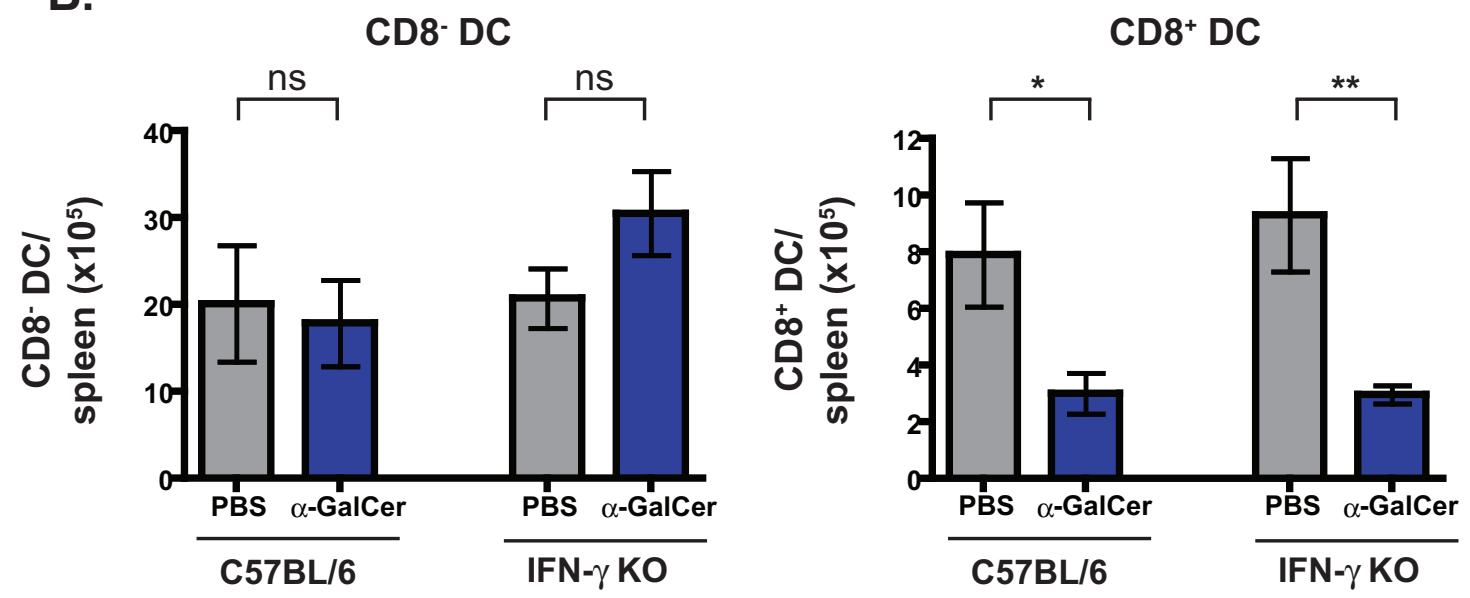

C.

CD8- DC
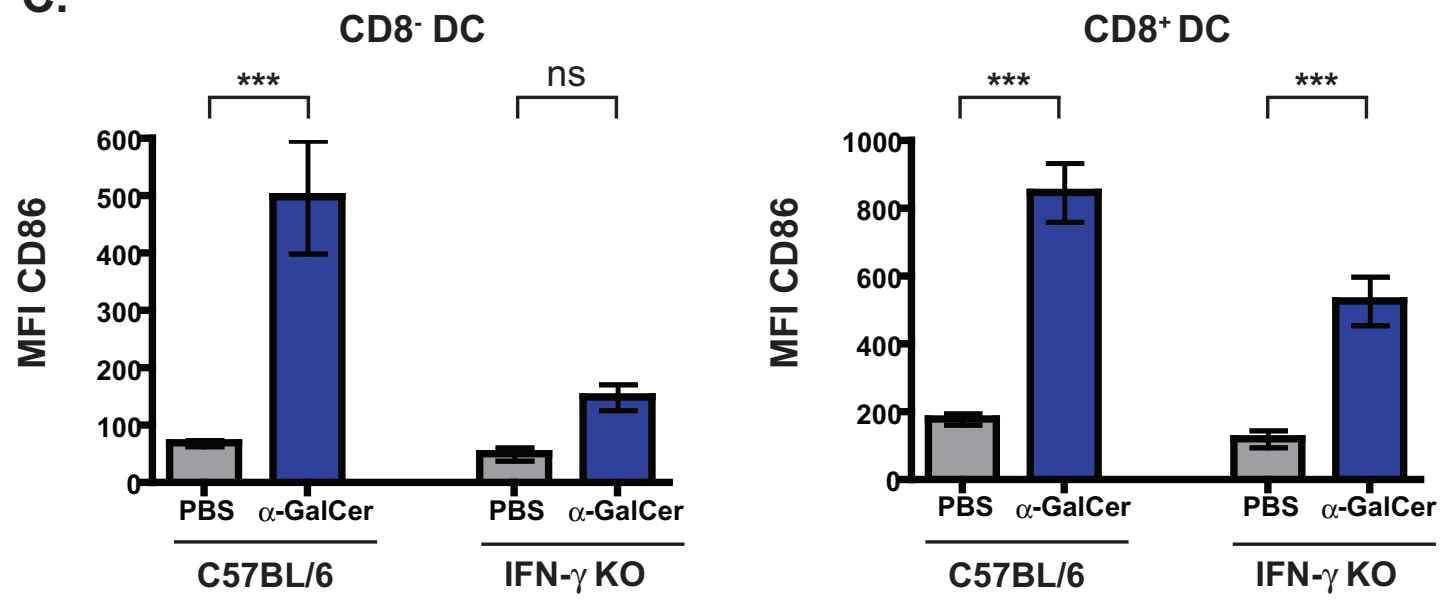


\subsubsection{TNF- $\alpha$ plays a role in the reduction of $\mathrm{CD8}^{+} \mathrm{DC}$ after $\alpha$-GalCer treatment}

Another possible candidate for cytokine mediated loss of the CD8 ${ }^{+}$DC was TNF- $\alpha$, which is produced by NKT cells (263) and DC (187) early after injection of $\alpha$-GalCer. In addition, TNF- $\alpha$ is also known to induce cell death (427). To investigate whether TNF- $\alpha$ was mediating the decrease in $\mathrm{CD}^{+} \mathrm{DC}$ a TNF- $\alpha$ neutralising antibody was used.

Firstly, it was important to confirm that the dose of TNF- $\alpha$ neutralising antibody used successfully blocked TNF- $\alpha$. To evaluate this C57BL/6 mice were given TNF- $\alpha$ neutralising antibody $20 \mathrm{hr}$ before $\alpha$-GalCer treatment, then $3 \mathrm{hr}$ after $\alpha$-GalCer treatment blood was collected and the level of serum TNF- $\alpha$ determined using the Bio-Plex ${ }^{\mathrm{TM}}$ system. Undetectable serum levels of TNF- $\alpha$ were found in mice treated with the TNF- $\alpha$ neutralising antibody (Figure 5.12 A). These data indicated that this method successfully blocked TNF- $\alpha$, at least transiently.

To determine if blocking TNF- $\alpha$ affected the loss of $\mathrm{CD} 8^{+} \mathrm{DC}$, mice were treated with anti-TNF- $\alpha$ followed by $\alpha$-GalCer treatment $20 \mathrm{hr}$ later. Forty-eight hr after $\alpha$-GalCer treatment splenocytes were assessed for the number $\mathrm{CD}^{+} \mathrm{DC}$ by flow cytometry. A greater number of splenic $\mathrm{CD} 8{ }^{+} \mathrm{DC}$ were recovered in $\mathrm{C} 57 \mathrm{BL} / 6$ mice treated with the TNF- $\alpha$ neutralising antibody compared to the control treated mice (Figure 5.12 B and C). No change in the number of CD8 ${ }^{-}$DC was seen after treatment with the TNF- $\alpha$ neutralising antibody (Figure 5.12 B and C). The cell surface expression of CD86 was also assessed to determine the activation status of the DC (Figure 5.13). In mice treated with the TNF- $\alpha$ neutralising antibody expression of CD86 on both DC subsets was markedly reduced compared to the controls (Figure 5.13). This is consistent with previously published data (187), where treatment of TNF KO mice resulted in reduced expression of CD86 on both $\mathrm{CD}^{+}$and $\mathrm{CD}^{-}$DC subsets. These results suggested that TNF- $\alpha$ played a role in the activation and the decrease of the splenic $\mathrm{CD}^{+} \mathrm{DC}$ subset after $\alpha$-GalCer treatment. 


\section{Figure 5.12 TNF- $\alpha$ plays a role in the decrease of $\mathrm{CD8}^{+} \mathrm{DC}$ after $\alpha$-GalCer treatment.}

C57BL $/ 6$ mice were injected i.p. with PBS or $10 \mathrm{mg} / \mathrm{kg}$ of anti-TNF- $\alpha$, treated i.v. with $\alpha$-GalCer $20 \mathrm{hr}$ later, and $48 \mathrm{hr}$ after $\alpha$-GalCer treatment spleens were harvested and analysed as described in Figure 5.1. (A) Levels of serum TNF- $\alpha 3$ hr after $\alpha$-GalCer treatment. TNF- $\alpha$ concentrations were determined using the Bio-Plex ${ }^{\mathrm{TM}}$ system. ND: not detectable. (B) Dot plots show flow cytometric analysis of spleen cells $48 \mathrm{hr}$ after $\alpha$-GalCer treatment, CD8- DC are indicated by a rectangle gate and the $\mathrm{CD}^{+}$DC are indicated by an oval gate. (C) Total number of CD8 ${ }^{-}$DC (left panel) and $\mathrm{CD}^{+} \mathrm{DC}$ (right panel) in the spleen $48 \mathrm{hr}$ after $\alpha$-GalCer treatment. Bars represent mean \pm range of 2 mice per group. These experiments were repeated with similar results. 
A.

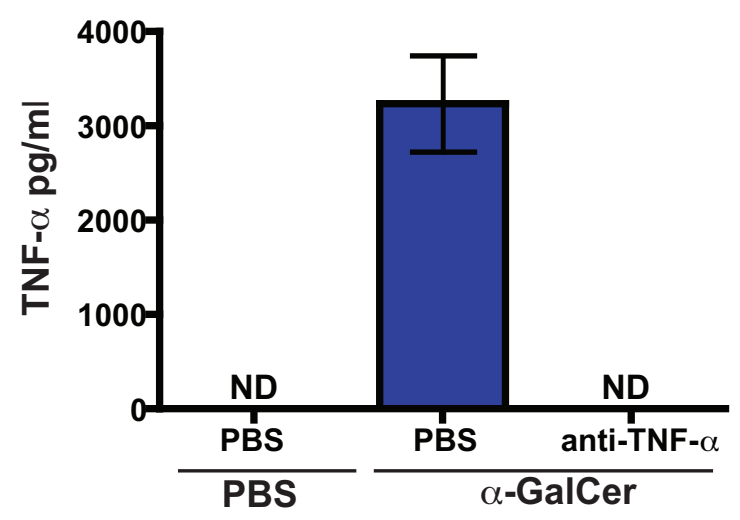

B.

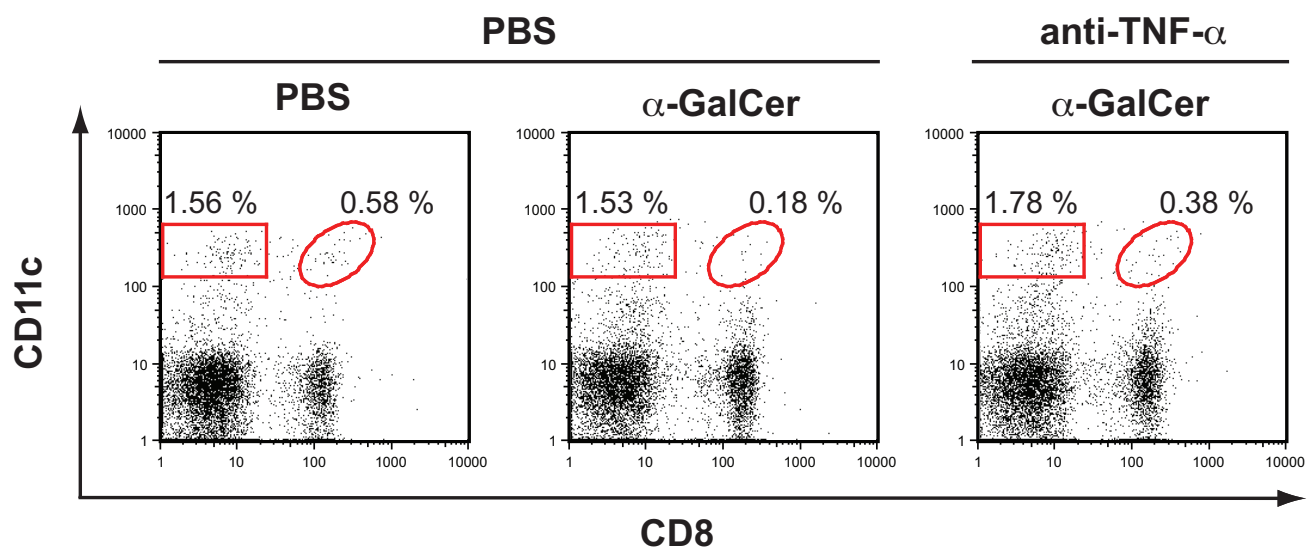

C.
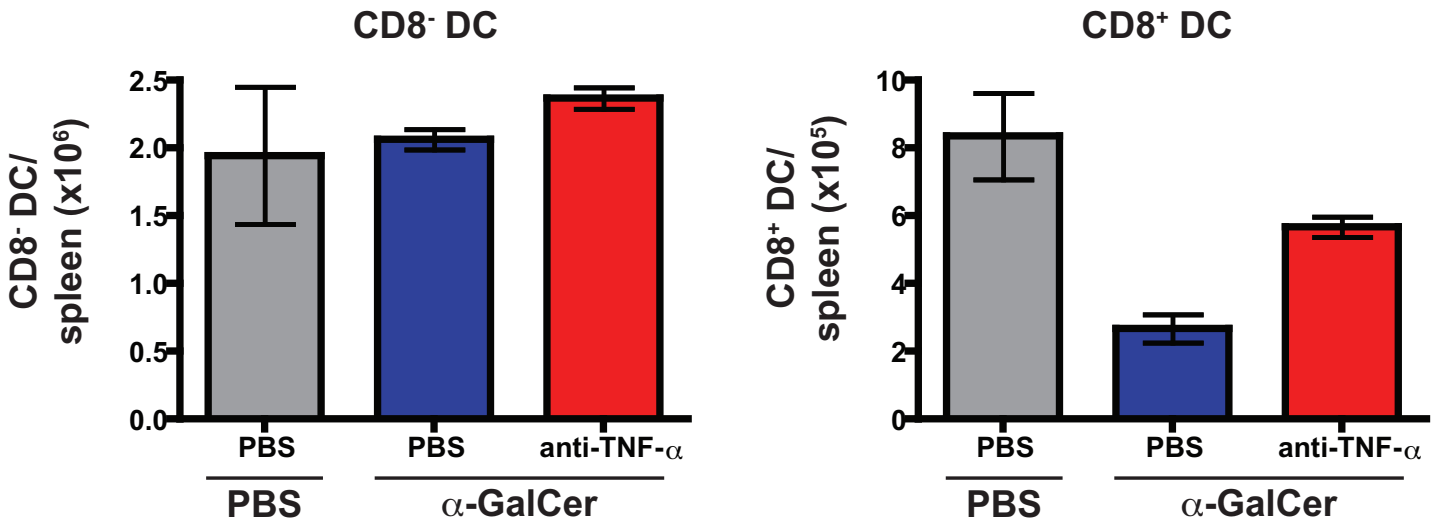

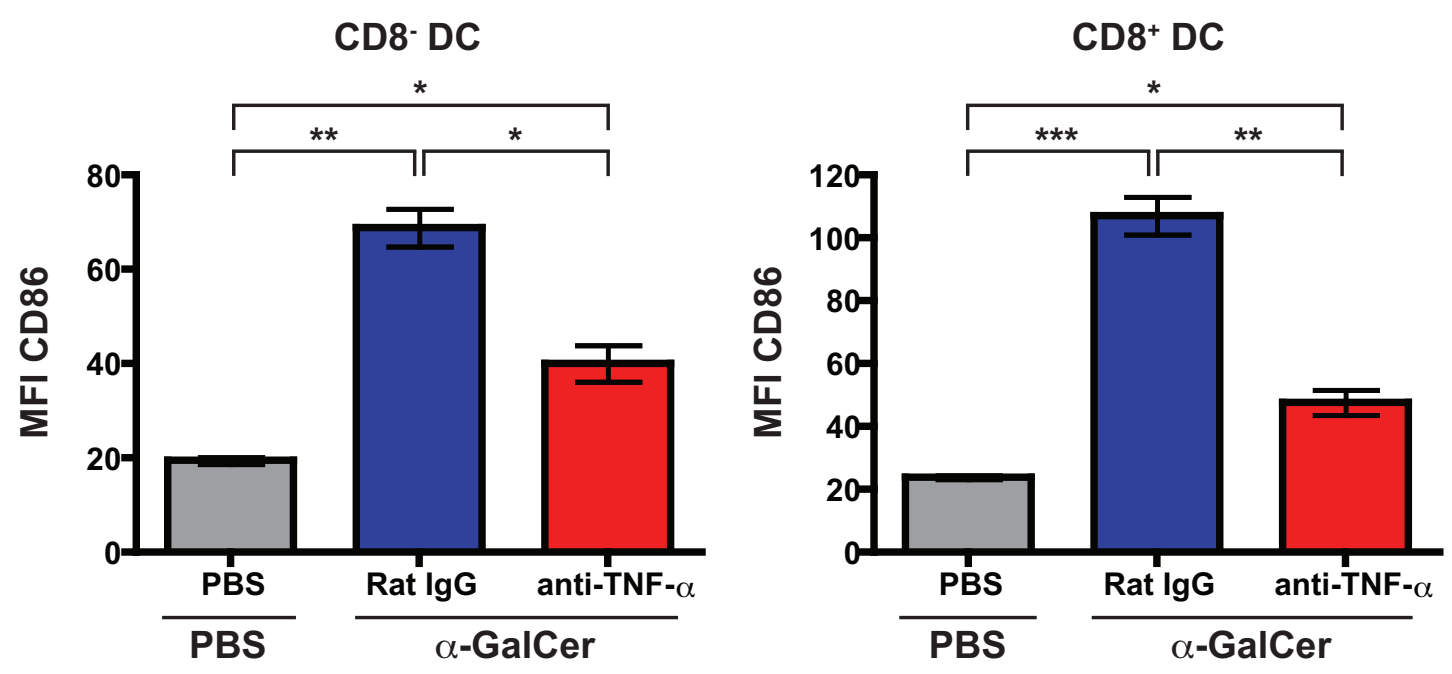

Figure 5.13 Treatment with a TNF- $\alpha$ neutralising antibody reduced the upregulation of CD86 on DC after $\alpha$-GalCer treatment.

C57BL/6 mice were injected i.p. with anti-TNF- $\alpha$ and treated i.v. with $\alpha$-GalCer as described in Figure 5.12. Expression of CD86 on CD8 ${ }^{-}$DC (left panel) and CD8 ${ }^{+}$DC (right panel) was examined in the spleen $24 \mathrm{hr}$ after $\alpha$-GalCer treatment. Bars represent mean \pm range of 2 mice per group. ***: $p<0.001, * *: 0.001<p<0.01$, and $*: p<0.05$ by one-way ANOVA with a Tukey post test. This experiment was repeated with similar results.

\subsubsection{Reduced numbers of $\mathrm{CD8}^{+} \mathrm{DC}$ after $\alpha$-GalCer treatment do not affect ova-specific immune responses}

Studies have reported that $\alpha$-GalCer acts as an adjuvant for $\mathrm{T}$ cell responses, as treatment with $\alpha$-GalCer and ova protein activated larger numbers of ova-specific $\mathrm{CD} 8^{+}$and $\mathrm{CD} 4^{+} \mathrm{T}$ cells compared to ova protein only $(319,320,325,326)$. DC are important for the adjuvant effect of $\alpha$-GalCer (187), thus a reduced number of $\mathrm{CD}^{+} \mathrm{DC}$ could affect the generation of $\mathrm{CD}^{+} \mathrm{T}$ cell responses. To assess whether the decrease in the $\mathrm{CD}^{+} \mathrm{DC}$ subset after $\alpha-$ GalCer treatment impacted on the $\mathrm{CD}^{+} \mathrm{T}$ cell response to protein antigen, C57BL/6 mice were treated with either $200 \mathrm{ng}$ or $1 \mathrm{ng}$ of $\alpha$-GalCer in conjunction with ova protein. The low $\alpha$-GalCer dose of $1 \mathrm{ng}$ was chosen because it caused no significant decrease in the number of $\mathrm{CD}^{+}$DC compared to $200 \mathrm{ng} \alpha$-GalCer but still induced some DC activation (Figure 5.6 A). To evaluate the ova-specific $\mathrm{CD}^{+} \mathrm{T}$ cell response after treatment with the 
different doses of $\alpha$-GalCer, the percentage of $\mathrm{CD} 8^{+} \mathrm{K}^{\mathrm{b}}$-ova $257-264{ }^{+} \mathrm{T}$ cells in the blood was examined 7 and 12 days after $\alpha$-GalCer treatment using a MHC I pentamer. The results showed that on day 7 the percentage of $\mathrm{CD}^{+} \mathrm{K}^{\mathrm{b}}-\mathrm{Ova}_{257-264}{ }^{+} \mathrm{T}$ cells was greater in mice treated with $200 \mathrm{ng} \alpha$-GalCer than in those treated with only $1 \mathrm{ng} \alpha$-GalCer and ova protein (Figure 5.14). However, 12 days after treatment the percentage of $\mathrm{CD} 8^{+} \mathrm{K}^{\mathrm{b}}$-ova 257 ${ }_{264}{ }^{+} \mathrm{T}$ cells was similar in mice treated with $200 \mathrm{ng}$ or $1 \mathrm{ng} \alpha$-GalCer (Figure 5.14). These data indicated that the activation status of DC during the initial priming of $\mathrm{CD} 8^{+} \mathrm{T}$ cells is more important than their numbers. Later responses did not appear to be as sensitive to the activation status of the DC compared to the requirement for the earlier responses. 


\section{Figure 5.14 The lower dose of $\alpha$-GalCer only affects the early ova-specific $\mathrm{CDB}^{+} \mathrm{T}$ cell}

\section{response.}

C57BL/6 mice were injected i.v. with either $1 \mathrm{ng}$ or $200 \mathrm{ng}$ of $\alpha$-GalCer and $200 \mu \mathrm{g}$ ova protein. Blood was collected from the tail vein 7 and 12 days later and labelled with anti-CD8 and $\mathrm{K}^{\mathrm{b}}$ ova $_{257-264}$ pentamer. Blood from mice injected with PBS was used as a negative control. The percentage of $\mathrm{CD}^{+} \mathrm{K}^{\mathrm{b}}$-ova $257-64{ }^{+}$cells was examined by flow cytometry. Dead $\left(\mathrm{PI}^{+}\right)$cells were excluded and lymphocytes were gated on the basis of their FSC vs. SSC properties. (A) Dot plots show total $\mathrm{CD}^{+}$cells, $\mathrm{CD} 8^{+} \mathrm{K}^{\mathrm{b}}$-ova $257-64^{+}$cells are highlighted by an oval gate and their percentage is shown. (B) Percentages of $\mathrm{CD} 8{ }^{+} \mathrm{K}^{\mathrm{b}}$-ova $257-64{ }^{+}$cells on day 7 (left panel) and 12 (right panel) after administration of $\alpha$-GalCer and ova protein. Each point represents an individual mouse and lines represent the mean. $* *: 0.001<p<0.01$ and ns: not significant by one-way ANOVA with a Tukey post test. 
A.

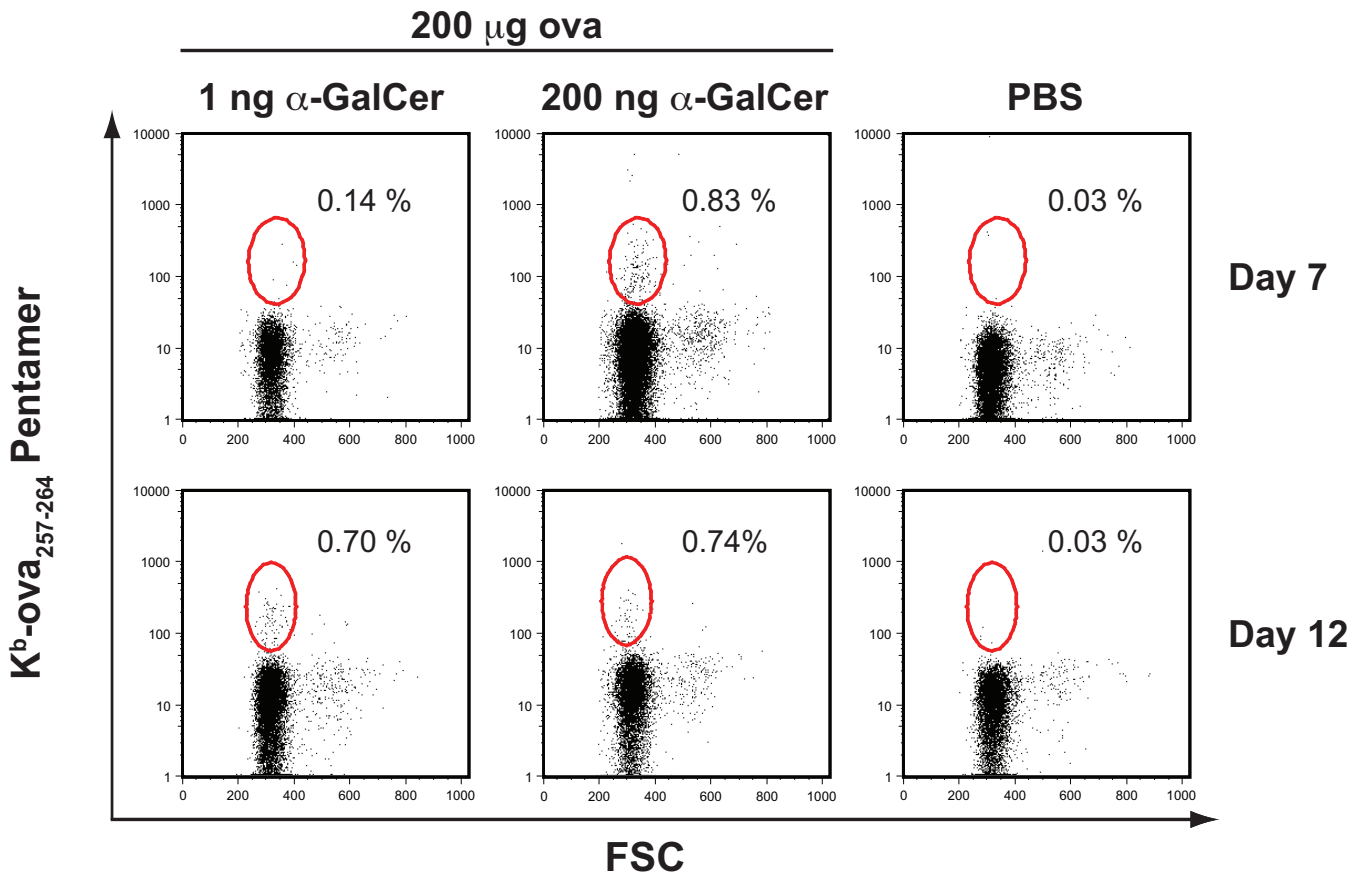

B.
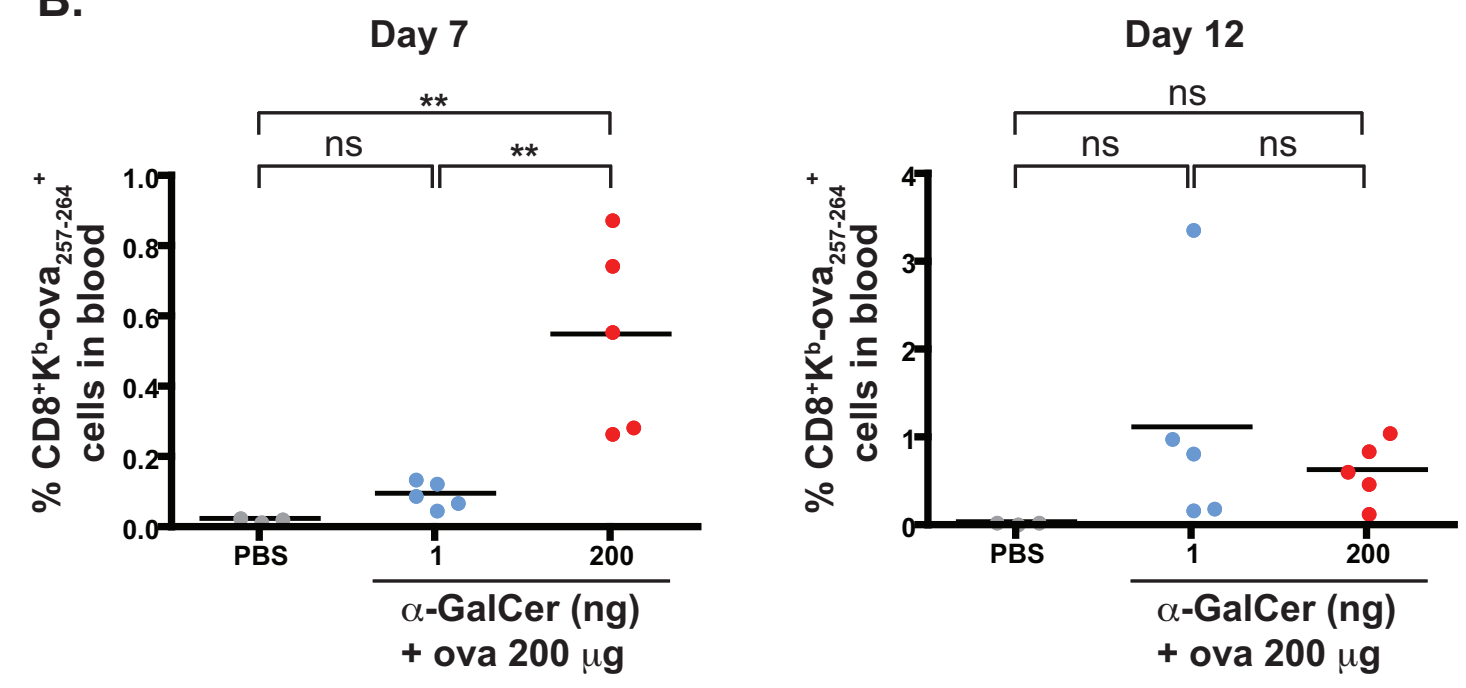
The use of $\alpha$-GalCer in conjunction with soluble ova has been shown to improve tumour immunity compared to ova alone $(187,319,320,325)$. To investigate whether the use of different doses of $\alpha$-GalCer impacted on tumour immunity, C57BL/6 mice were treated s.c. with the B16.ova melanoma tumour 30 days after treatment with either $1 \mathrm{ng}$ or $200 \mathrm{ng}$ $\alpha$-GalCer and ova protein. Tumour growth was then monitored over time. Mice treated with either $1 \mathrm{ng}$ or $200 \mathrm{ng} \alpha$-GalCer along with ova protein developed tumours approximately 25 days later compared to the control group (Figure 5.15). This reflects the results reported in Figure 5.14, where the percentages of ova-specific T cells were similar in mice treated with $1 \mathrm{ng}$ or $200 \mathrm{ng} \alpha$-GalCer on day 12 . Thus in this prophylactic setting the different doses of $\alpha$-GalCer did not affect tumour protection.

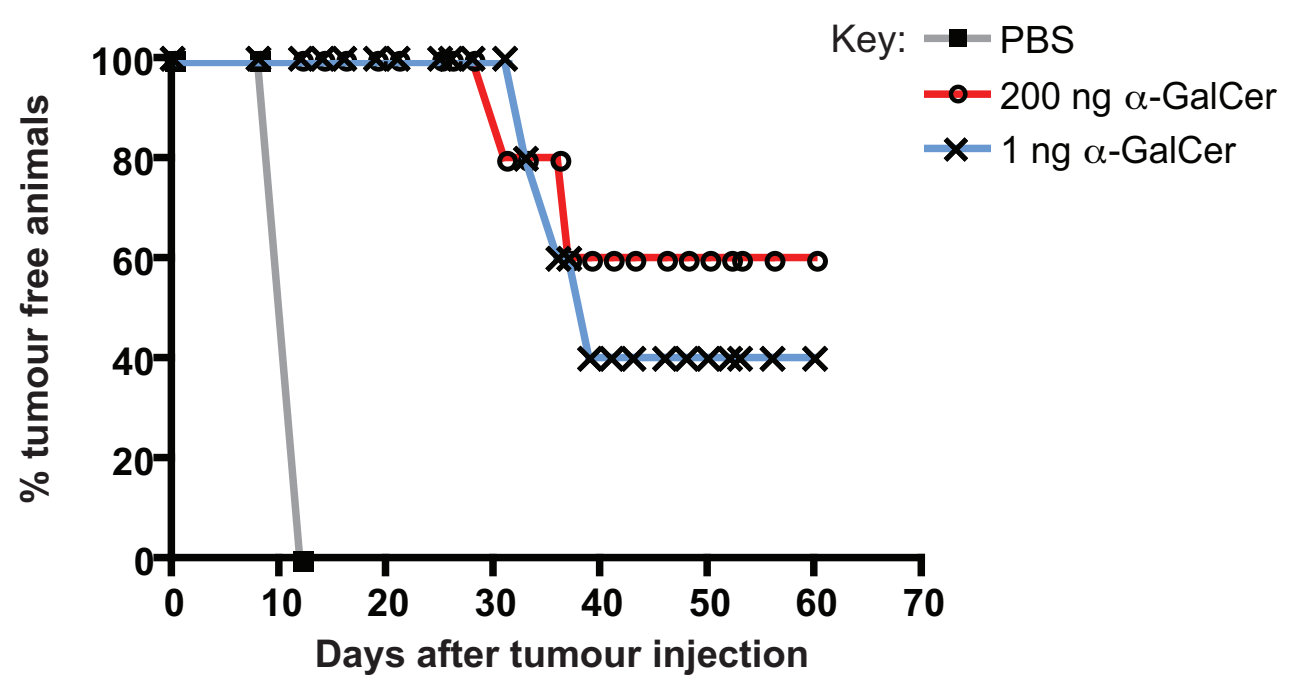

Figure 5.15 Administration of ova together with low or high doses of $\alpha$-GalCer elicits similar tumour protection.

C57BL/6 mice were injected i.v. with either 1 or $200 \mathrm{ng} \alpha$-GalCer and $200 \mu \mathrm{g}$ ova protein. As a negative control mice were injected i.v. with PBS. Thirty days later mice were injected s.c. with $1 \times 10^{5}$ B16.ova tumour cells in the left flank and tumour growth was monitored. A tumour was scored as positive when the product of bisecting diameters exceeded $4 \mathrm{~mm}^{2}$. Each point represents the percentage of tumour free animals with 4-5 mice per group. 
Administration of a TNF- $\alpha$ neutralising antibody maintained a higher number of $\mathrm{CD}^{+} \mathrm{DC}$ after $\alpha$-GalCer treatment. To determine if administration of a TNF- $\alpha$ neutralising antibody impacted on the ova-specific $\mathrm{CD} 8^{+} \mathrm{T}$ cell response, $\mathrm{C} 57 \mathrm{BL} / 6$ mice were adoptively transferred with purified OT-IxB6 congenic $\mathrm{CD} 8^{+} \mathrm{LN}$ cells, treated with anti-TNF- $\alpha$ antibody $2 \mathrm{hr}$ later, and treated with $\alpha$-GalCer and ova after a further $20 \mathrm{hr}$. At specific time points after treatment the number of transferred cells in the blood was determined using a MHC I pentamer. Seven days after treatment, mice that were treated with the TNF$\alpha$ neutralising antibody before $\alpha$-GalCer treatment had a statistically greater number of transferred cells present in the blood (Figure 5.16). However, this increase in transferred cells was not maintained over time. At the last time point measured mice treated with the TNF- $\alpha$ neutralising antibody showed a significant decrease in the number of transferred cells compared to the control treated group (Figure 5.16 B). These data indicated that treatment with the TNF- $\alpha$ neutralising antibody improved the early but not the late $\mathrm{CD}^{+} \mathrm{T}$ cell response. Furthermore, these results suggest that low doses of $\alpha$-GalCer or anti-TNF- $\alpha$ treatment, which maintained a higher number of $\mathrm{CD} 8^{+} \mathrm{DC}$, and resulted in less activated $\mathrm{DC}$, did not lead to improved $\mathrm{CD}^{+} \mathrm{T}$ cell responses. 
Figure 5.16 Blocking TNF- $\alpha$ during treatment with $\alpha$-GalCer and ova improves the early but not the late $\mathrm{CDB}^{+} \mathrm{T}$ cell response.

$\mathrm{CD}^{+}$cells were purified from the LN of a OT-IxB6 congenic mouse using magnetic separation and $5 \times 10^{3}$ cells were adoptively transferred into C57BL/6 mice. Two hr later mice were injected i.p. with $10 \mathrm{mg} / \mathrm{kg}$ Rat IgG or anti-TNF- $\alpha$, then treated i.v. with $\alpha$-GalCer $20 \mathrm{hr}$ later. At the specific time points indicated blood was collected from the tail vein and examined by flow cytometry for the presence of transferred cells $\left(\mathrm{CD}^{+} \mathrm{CD} 45.1^{+}\right)$. Dead $\left(\mathrm{PI}^{+}\right)$cells were excluded and lymphocytes were gated on the basis of their FSC vs. SSC properties. (A) The percentage of $\mathrm{CD} 8{ }^{+} \mathrm{CD} 45.1^{+}$cells in the blood 7 days after treatment with $\alpha$-GalCer and ova protein. Each point represents an individual mouse and lines represent the mean. (B) Time course of the percentages of $\mathrm{CD} 8{ }^{+} \mathrm{CD} 45.1^{+}$cells in blood after treatment with $\alpha$-GalCer and ova protein. Each point represents mean \pm SD of 4-5 mice per group. The statistical analyses in (B) refer to the $\alpha$-GalCer and ova protein treated groups $* *: 0.001<p<0.01, *: p<0.05$ and ns: not significant by two way ANOVA with a Tukey post test. 
A.

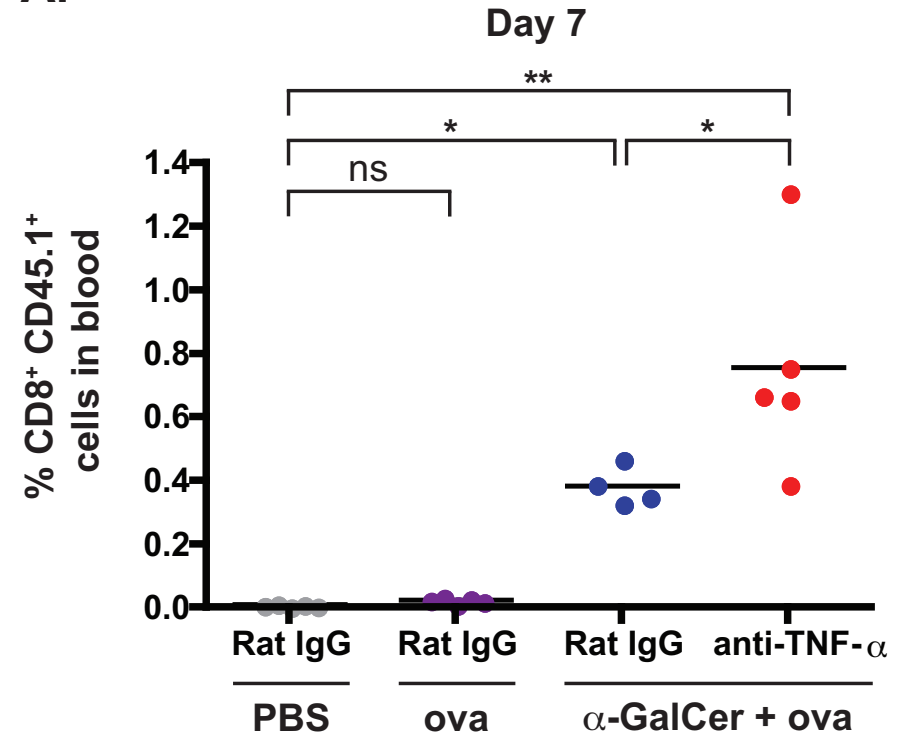

B.

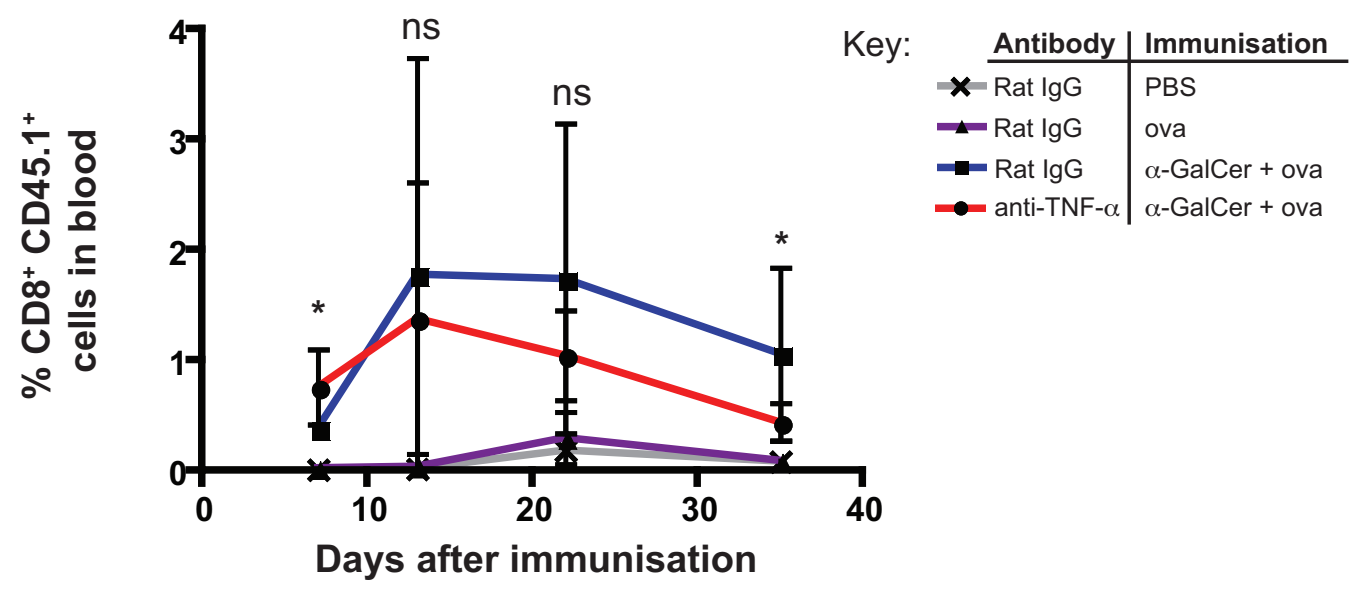




\subsubsection{Neutralisation of TNF- $\alpha$ leads to reduced NKT cell activation}

In mice treated with the TNF- $\alpha$ neutralising antibody, DC were less activated and expressed lower levels of CD86 (Figure 5.13). To determine whether NKT cell activation was affected by TNF- $\alpha$ neutralisation, the serum level of cytokines associated with NKT cell activation were measured. Treatment with the TNF- $\alpha$ neutralising antibody led to significantly reduced serum levels of IL-4, IFN- $\gamma$ and IL-12p70 (Figure 5.17 A and C-D). These cytokines are produced by different cell populations, with IL-4 being produced by NKT cells, IL-12p70 by DC, while IFN $\gamma$ is produced by both NKT cells and NK cells. Thus the presence of TNF- $\alpha$ seems to be critical in the activation of NKT cells, DC and NK cells. 
A.

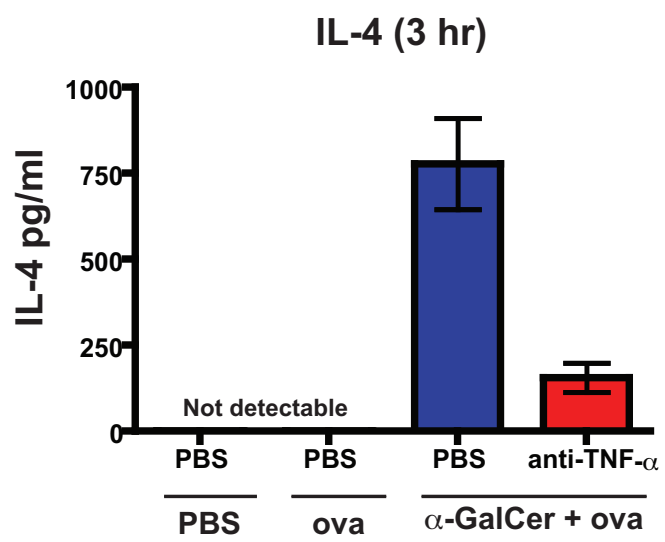

C.

IFN- $\gamma(6 \mathrm{hr})$

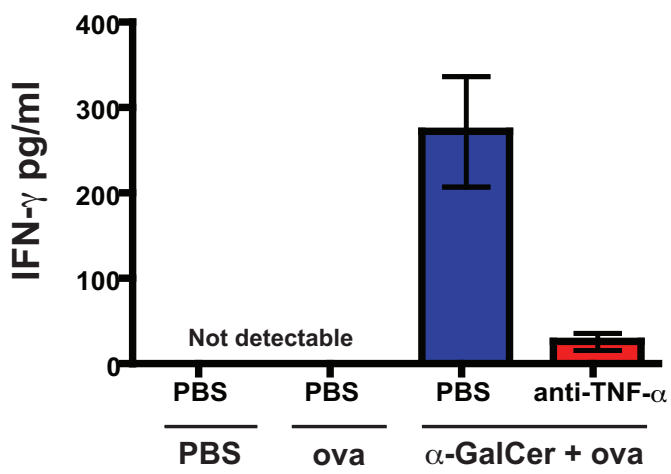

B. TNF- $\alpha(3 \mathrm{hr})$

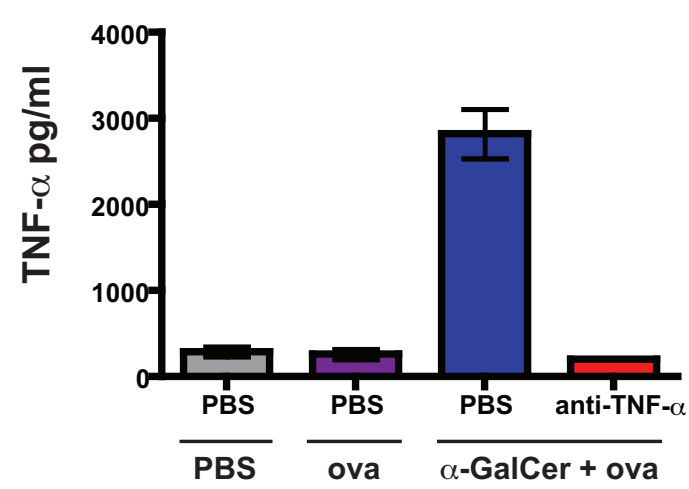

D.

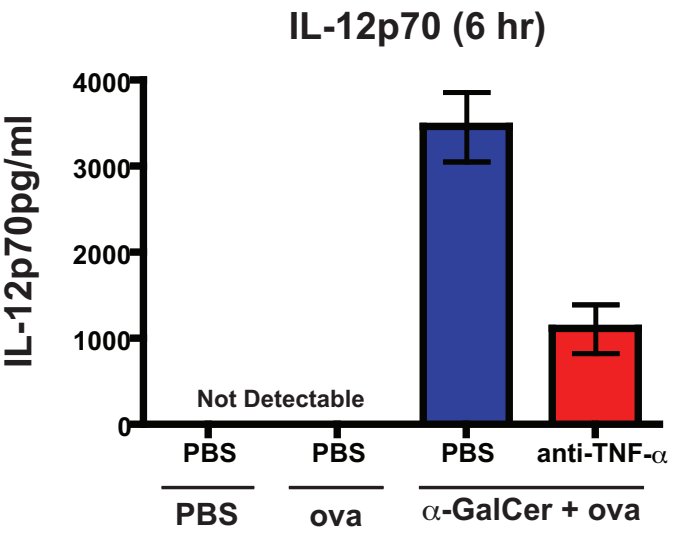

Figure 5.17 Treatment with anti-TNF- $\alpha$ leads to reduced serum cytokine levels after $\alpha$ -

\section{GalCer treatment.}

C57BL/6 mice were injected i.p. with anti-TNF- $\alpha$ and treated i.v. with $\alpha$-GalCer and ova protein as described in Figure 5.12. Three and six hr after $\alpha$-GalCer and ova protein treatment the levels of serum TNF- $\alpha$, IL-4, IL-12p70 and IFN- $\gamma$ were determined using the Bio-Plex ${ }^{\mathrm{TM}}$ system. Bars represent mean \pm SD of 5 mice per group. This experiment was repeated with similar results. 


\subsection{Discussion}

The aim of this chapter was to determine the mechanism of the loss of CD8 $8^{+}$DC after $\alpha-$ GalCer treatment, and its potential effects on immune responses. It was discovered that the decrease in $\mathrm{CD}^{+} \mathrm{DC}$ was transiently induced by both $\alpha$-GalCer and its analogue $\mathrm{OCH}$, and required the presence of NKT cells. Neutralisation of TNF- $\alpha$ provided evidence that TNF- $\alpha$ was a key mediator in the reduction of $C D 8^{+}$DC after $\alpha$-GalCer treatment. DC depletion was intimately linked to activation, and preventing the loss of $\mathrm{CD} 8^{+} \mathrm{DC}$ also decreased DC activation, and did not improve the generation of $\mathrm{CD}^{+} \mathrm{T}$ cell response to a co-administered protein antigen.

Firstly, it was established that loss of splenic $\mathrm{CD} 8^{+} \mathrm{DC}$ after $\alpha$-GalCer treatment was due to cell loss, and not to down regulation of the CD8 molecule. This was determined by using cell markers other than CD8 to indirectly establish the presence of $\mathrm{CD} 8^{+} \mathrm{DC}$ in $\alpha$ GalCer treated mice. In the steady state, $\mathrm{CD}^{+}$DC express DEC 205 and lack expression of CD11b (59). Unfortunately, DEC 205 was found not to be suitable to the study of $\alpha$ GalCer treatment. Upon treatment with $\alpha$-GalCer CD8- DC appeared to upregulate expression of DEC 205, a finding also reported by other authors (187). Similarly, expression of DEC 205 and CD11b on $\mathrm{CD}^{-}$and $\mathrm{CD}^{+} \mathrm{DC}$, respectively, are increased after overnight culture, indicating that DEC 205 and CD11b are not stable DC lineage markers (59). Other markers that gave more reliable results were Langerin (421, 423), and CD24, which are both reported to be expressed on $\mathrm{CD}^{+} \mathrm{DC}(58,424)$. The results described in Figures 5.2 and 5.3, where the expression of Langerin and CD24 was investigated after $\alpha$-GalCer treatment, showed a decrease in the $\mathrm{CD}^{+} \mathrm{DC}$ subset after $\alpha$ GalCer treatment, indicating that the DC subset was depleted after $\alpha$-GalCer treatment.

It has been recently recognised that the $\mathrm{CD} 8^{+} \mathrm{DC}$ are a heterogenous cell subset. They have been divided based on the expression of Langerin (CD207) and integrin $\alpha_{\mathrm{E}}$ (Itgae) also known as CD103, into two groups, either CD207 ${ }^{+} \mathrm{CD} 103^{+}$or $\mathrm{CD} 207^{-} \mathrm{CD} 103^{-}$. It was observed that the $\mathrm{CD} 207^{+} \mathrm{CD} 103^{+} \mathrm{DC}$ are superior in their ability to cross present soluble antigen and phagocytose apoptotic bodies (420). These data are consistent with an earlier report that demonstrates that not all the $\mathrm{CD} 8^{+} \mathrm{DC}$ can cross present cellular antigen indicating heterogeneity within the population (417). Furthermore, TLR ligand stimulation 
caused disappearance of only the $\mathrm{CD}_{103^{+}} \mathrm{DC}$ in vivo, which resulted in reduced tolerance induction (420). Additionally, another recent paper showed that the $\mathrm{CD} 8^{+} \mathrm{CD} 207^{+}$ population disappeared after in vivo TLR ligand stimulation (428). Similar results were observed when Langerin-EGFP mice were treated with $\alpha$-GalCer, as only the CD8 ${ }^{+} \mathrm{EGFP}^{+}$ DC declined in cell number compared to the $\mathrm{CD} 8^{+} \mathrm{EGFP}^{-} \mathrm{DC}$ (data not shown). Thus it would be of interest to further characterise the $\mathrm{CD} 8^{+} \mathrm{DC}$ that are lost after $\alpha$-GalCer treatment by investigating the expression of CD103.

As discussed in section 1.1.3, there is evidence indicating that the different murine splenic cDC subsets are functionally distinct. In particular, $\mathrm{CD} 8^{+} \mathrm{DC}$ express a different TLR repertoire from all other murine spleen DC subsets (65), and reduced levels of the antiapoptotic molecule Bcl-2 (429), suggesting that after recognition of certain stimuli CD8 ${ }^{+}$ DC may be more sensitive to apoptosis than $\mathrm{CD}^{-}$DC. In support of this, data presented in this thesis demonstrated that $\mathrm{CD} 8^{+} \mathrm{DC}$ were selectively decreased after treatment with $\alpha$ GalCer. Loss of $\mathrm{CD}^{+} \mathrm{DC}$ has also been reported during a range of different infections (430-433). Taken together these data suggest that $\mathrm{CD} 8^{+} \mathrm{DC}$ are more sensitive and possibly more prone to apoptosis than $\mathrm{CD}^{-} \mathrm{DC}$.

It is possible that the differential response of $\mathrm{CD}^{+}$and $\mathrm{CD}^{-} \mathrm{DC}$ to $\alpha$-GalCer treatment is due to DC receiving multiple signals, some of which lead to activation of all DC, while others lead to the survival or death of specific DC subsets. Experiments attempted to separate the activation and survival/death signals by injecting decreasing doses of $\alpha$ GalCer, and by using the $\alpha$-GalCer analogue $\mathrm{OCH}$, which has a lower binding affinity for mouse NKT cells (434) and induces a differential cytokine response compared to $\alpha$-GalCer (305). Conditions where DC activation and depletion were separated could not be identified in C57BL/6 mice, suggesting that $\mathrm{CD} 8^{+}$DC loss was most consistent with activation-induced cell death, as already described by others in DC responding to LPS (61, 420). Against this hypothesis, a-GalCer injection induced weak upregulation of CD86 in B6.lpr mice, but some CD8+ DC loss was still observed. This suggests that DC activation and CD8+ DC loss can be dissociated at least in some cases. No further studies were carried out to characterize the response of B6.lpr mice to a-GalCer, and determine whether additional aspects of this response might also have been defective. 
$\mathrm{CD}^{+}$DC express more CD1d and could preferentially interaction with NKT cells. To establish if direct contact between $\mathrm{CD}^{+} \mathrm{DC}$ and NKT cell was required for the decline in $\mathrm{CD}^{+}$DC, C57BL/6 mice were injected with BMDC loaded with $\alpha-G a l C e r$. It was assumed that in this set-up the only DC able to present $\alpha$-GalCer to the NKT cells were the adoptively transferred BMDC. Thus if a reduction in the number of resident splenic $\mathrm{CD} 8^{+}$ DC was observed this would indicate that the loss of $\mathrm{CD}^{+} \mathrm{DC}$ was not mediated by direct NKT cell contact. However, a decrease in resident splenic CD ${ }^{+} \mathrm{DC}$ was also observed when CD1 KO BMDC were loaded with $\alpha$-GalCer and adoptively transferred into C57BL/6 mice. Since CD1 KO BMDC cannot directly present $\alpha-G a l C e r$, this result must be due to cross presentation of the injected BMDC, or of released $\alpha-G a l C e r$, by resident splenic DC (Figure 5.18), presumably the $\mathrm{CD} 8^{+}$DC. Other reports have found that lipids can be cross-presented. Tumour cells loaded with $\alpha$-GalCer and injected into mice can be taken up by host DC to induce activation of NKT cells (435). Similarly, when human melanoma cells that lack expression of any isoform of human CD1 and express the ganglioside, GD3, were injected into mice, activated GD3 reactive NKT cells were found, indicating cross-presentation of GD3 by murine APCs in vivo (436). In conclusion, the use of $\alpha$-GalCer on BMDC did not provide any firm evidence on whether the decrease in $\mathrm{CD}^{+} \mathrm{DC}$ involved the formation of direct contacts between NKT cells and DC. 


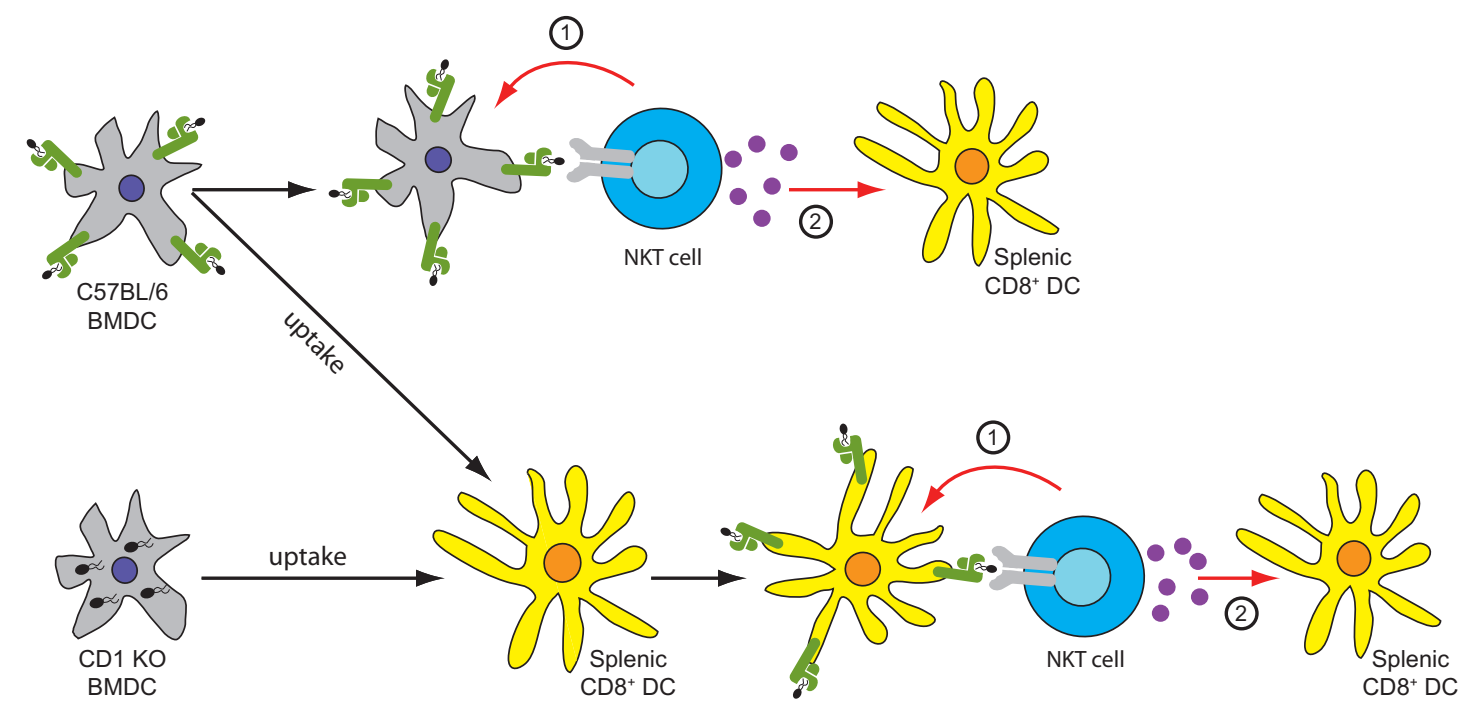

Figure 5.18 A schematic diagram of the mechanisms of $\alpha$-GalCer presentation after adoptive transfer of BMDC loaded with $\alpha$-GalCer.

A schematic diagram of the possible mechanisms that lead to decrease in splenic $\mathrm{CD} 8^{+} \mathrm{DC}$ after adoptive transfer of BMDC. BMDC can directly present $\alpha$-GalCer to NKT cells, or alternatively resident splenic DC can take up the BMDC and present $\alpha$-GalCer presented on their CD1d molecules. The uptake of BMDC by splenic DC allows NKT cells to directly interact with them. NKT cells can then directly target the DC through cell mediated contact pathways (1), or alternatively, the NKT cells can release cytokines that then act directly on the DC or through other cell types (2).

Since no strong experimental evidence was generated to suggest that the decline in $\mathrm{CD} 8^{+} \mathrm{DC}$ was mediated by a contact dependent mechanism, the possibility that a soluble mediator was causing the loss of $\mathrm{CD} 8^{+} \mathrm{DC}$ was considered. One possible candidate was the cytokine IFN- $\gamma$. NKT cells rapidly produce high levels of IFN- $\gamma$ upon stimulation with $\alpha$-GalCer (263). However, a decrease in $\mathrm{CD}^{+} \mathrm{DC}$ was also seen after treatment with the glycolipid $\mathrm{OCH}$, which preferentially induces IL-4 production by NKT cells and little IFN- $\gamma(305-307)$, suggesting that IFN- $\gamma$ was not involved in the loss of the $\mathrm{CD} 8^{+} \mathrm{DC}$. This conclusion was also supported by experiments where IFN- $\gamma$ KO mice treated with $\alpha$-GalCer showed a decline in the number of $\mathrm{CD} 8^{+} \mathrm{DC}$ in spleen, as observed in $\mathrm{C} 57 \mathrm{BL} / 6$ mice. Thus these data 
demonstrated that IFN- $\gamma$ was not the soluble mediator involved in the decrease of the CD $8^{+}$ DC after $\alpha$-GalCer treatment.

Another possible candidate for mediating the decline in $\mathrm{CD}^{+}$DC was TNF- $\alpha$. TNF- $\alpha$ is a highly pleiotropic, pro-inflammatory cytokine that affects cellular proliferation and differentiation, as well as activation of apoptosis $(220,437)$. A study by Smyth and Sedgwick showed that TNF- $\alpha$ released from antigen activated $C D 8^{+} \mathrm{T}$ cells was an important soluble mediator of bystander lysis (438). Furthermore, both NKT cells (263) and DC (187) have been shown to produce TNF- $\alpha$ within a few hours of $\alpha$-GalCer stimulation. Consequently, high levels of TNF- $\alpha$ after $\alpha$-GalCer treatment could lead to cell death. When TNF- $\alpha$ was blocked using a neutralising antibody the number of $\mathrm{CD} 8^{+} \mathrm{DC}$ did not decline as dramatically as in the control group. This is consistent with previously published data where $\mathrm{CD} 8^{+} \mathrm{DC}$ were selectively lost after an oral Salmonella infection in WT mice, but were resistant to cell death in TNFR1 deficient mice (433). Furthermore, TNF- $\alpha$ can only induce apoptosis in cells with low levels of anti-apoptotic proteins (439), which is consistent with the lower mRNA levels of Bcl-2 present in $\mathrm{CD}^{+}$DC (429). Taken together these data suggest that TNF- $\alpha$ selectively mediates the disappearance of $\mathrm{CD} 8^{+} \mathrm{DC}$ during different immune responses.

The loss of CD $8^{+}$DC after $\alpha$-GalCer treatment involved the cytokine TNF- $\alpha$. TNF- $\alpha$ is primarily produced as a type II transmembrane protein arranged in stable homotrimers. This membrane intregrated form (mTNF) can then be proteolytic cleaved to release the soluble homotrimeric cytokine (sTNF) (220). There is evidence to suggest that mTNF has a role in cytotoxicity $(440,441)$. In the experiments described in this Chapter, it is not possible to distinguish whether it is mTNF or STNF that contributes most significantly to the loss of CD8 ${ }^{+}$ DC. Thus until this can be accurately assessed it is possible that the loss of CD $8^{+} \mathrm{DC}$ is at least partly mediated by direct cell contact with the NKT cells. However, as the administration of a TNF- $\alpha$ neutralising antibody inhibited the activation of both $\mathrm{CD}^{+}$and CD8 $8^{-} \mathrm{DC}$, it would point towards sTNF, rather than mTNF being involved in the loss of $\mathrm{CD}^{+} \mathrm{DC}$. Though, if the depletion of $\mathrm{CD}^{+}$DC observed after $\alpha$-GalCer treatment was due to the direct effects of sTNF, it is somewhat surprising that other subsets of DC, such as the CD8- DC subset, were not similarly depleted. 
The involvement of TNF- $\alpha$ in the loss of the $\mathrm{CD} 8^{+} \mathrm{DC}$ would suggest that the $\mathrm{DC}$ are dying by apoptosis induced by TNF signalling, however, no DC death could be directly demonstrated in our studies. Studies of LPS-treated mice observed upregulation of CD80/CD86 and MHC II on splenic DC (61), followed by their death by apoptosis (145). Ex vivo labelling of DC with the viability dyes Annexin V and PI was conducted to verify whether apoptosis of $\mathrm{CD}^{+} \mathrm{DC}$ was occurring, however, the data was not informative due to high background labelling in the control group (data not shown). New technology now exists where dyes can be injected in vivo followed by flow cytometric analysis to evaluate the level of early stage apoptotic cell death. These experiments would clarify whether $\mathrm{CD} 8^{+} \mathrm{DC}$ are dying by apoptosis after $\alpha$-GalCer treatment.

Since no direct experimental evidence was available to show that the $\mathrm{CD} 8^{+} \mathrm{DC}$ were undergoing cell death after $\alpha$-GalCer treatment, it was possible that the observed loss was due to DC migration out of the spleen into other tissues. However, an extensive literature search did not find any reports of DC migrating out of the spleen. In fact, it has been reported that after LPS treatment CD11 ${ }^{+}$DC migrated from the marginal zone to the $\mathrm{T}$ cell zone within the spleen and then rapidly died by apoptosis within the T cell zone $(61,145)$. More recently, it has been reported that after treatment with poly(I:C) or LPS Langerin expressing $\mathrm{CD} 8^{+} \mathrm{DC}$ redistribute from the marginal zone to the $\mathrm{T}$ cell zone. This was followed by the disappearance of Langerin staining, which was presumed to be cell death of the Langerin expressing DC (428). Thus these data would provide support that perhaps the $\mathrm{CD} 8^{+} \mathrm{DC}$ are not migrating out of the spleen after $\alpha$-GalCer treatment but instead dying by apoptosis.

As well as TNF- $\alpha$ playing a role in the decrease of $\mathrm{CD}^{+} \mathrm{DC}$ after $\alpha$-GalCer treatment, it appears that TNF- $\alpha$ was also important for the interaction between DC and NKT cell. Administration of a TNF- $\alpha$ neutralising antibody resulted in reduced expression of CD86 and IL-12p70 production by DC, while reduced serum levels of IFN- $\gamma$ and IL-4 indicated reduced NKT cell activation. This reduced activation of both DC and NKT cell confirms that the activation of NKT cells leads to reciprocal activation of DC $(319,320,425)$. There is evidence to suggest that TNF- $\alpha$ can help enhance IL-12p70 production by DC, which is important for the generation of effective $\mathrm{CD}^{+} \mathrm{T}$ cell responses (179) and is required for the production of 
IFN- $\gamma$ by NKT cells (405). Treatment with TNF- $\alpha$ has shown to induce a small antigen specific $\mathrm{CD}^{+} \mathrm{T}$ cell response in the presence of CD40 signalling (187) and TNF signalling was required for the production of IL-12p70 during DC-NK cell interactions (442). Thus these data illustrate that TNF- $\alpha$ plays a role in enhancing IL-12p70 production by DC and is important for the interaction between DC and NKT cell.

DC are critical to the adjuvant effect of $\alpha$-GalCer (187). Thus a reduced number of DC could reduce the adjuvant effect of $\alpha$-GalCer. Recently, others have shown that the loss of $C D 8^{+} \mathrm{DC}$ affected tolerance induction and $\mathrm{T}$ cell immunity $(417,420)$. In contrast, methods used in this study to retain a greater number of $\mathrm{CD} 8^{+} \mathrm{DC}$ did not improve the $\mathrm{CD} 8^{+} \mathrm{T}$ cell response after treatment with $\alpha$-GalCer and ova protein, suggesting that the loss of the $\mathrm{CD} 8^{+} \mathrm{DC}$ does not profoundly affect the $\mathrm{CD} 8^{+} \mathrm{T}$ cell response. This result is consistent with the requirement for only a brief period of antigenic stimulation for the generation of strong $\mathrm{CD} 8^{+} \mathrm{T}$ cell responses. However, the methods employed in this study to retain a greater number of $\mathrm{CD} 8^{+} \mathrm{DC}$ after $\alpha$ GalCer treatment also led to a reduced activation status of the DC. Co-stimulation through CD28:CD86/CD80 signalling has been shown to be important for $\mathrm{T}$ cell activation and differentiation (110), thus reduced expression of CD86 on the DC would result in reduced costimulation provided to the $\mathrm{CD} 8^{+} \mathrm{T}$ cells. This is likely to explain why a greater number of $\mathrm{CD}^{+} \mathrm{DC}$ did not improve the $\mathrm{CD} 8^{+} \mathrm{T}$ cell response and illustrates that the $\mathrm{DC}$ activation status is at least as important as absolute number. Thus perhaps if a greater number of $\mathrm{CD} 8^{+}$ DC could be retained after $\alpha$-GalCer treatment along with an activated phenotype then an improved $\mathrm{CD}^{+} \mathrm{T}$ cell response might be generated.

The powerful adjuvant effect of $\alpha$-GalCer comes from the activation of NKT cells rapidly producing a large array of cytokines leading to the trans-activation of many cell types, including DC and B cells. Studies in this Chapter focused on the activation of DC after $\alpha-$ GalCer treatment, but as discussed in Chapter 1, B cells also express CD1d and can present $\alpha$ GalCer on their cell surface (91-93). Reports have demonstrated that NKT cell activation results in B cells proliferation and antibody secretion (327-333). However, others discovered that B cells poorly activate NKT cells to produce only Th2 cytokines (391) and were shown to be responsible for the induction of anergic NKT cells (406). Additionally, B cells appeared to 
have a suppressive role in DC mediated NKT cell activation (391). Though, more recently it has been suggested that marginal zone B cells play a role in NKT cell activation $(333,334$, 443 ) and were found to amplify the DC mediated activation of NKT cells (443). Thus, perhaps B cells affect the decline of $\mathrm{CD}^{+} \mathrm{DC}$ after $\alpha$-GalCer treatment. Therefore, it would be of interest to carry out further investigations using a mouse model deficient in B cells to assess whether B cells have a role in the loss of this DC subset after $\alpha$-GalCer treatment.

\subsubsection{Summary}

Treatment $\alpha$-GalCer results in the NKT cell-dependent depletion of splenic CD8 ${ }^{+} \mathrm{DC}$. The precise mechanism leading to the decline of $\mathrm{CD}^{+} \mathrm{DC}$ was not definitively established, but appeared to involve the cytokine TNF- $\alpha$. The loss of CD8 ${ }^{+} \mathrm{DC}$ was transient and administration of the NKT cell ligand $\mathrm{OCH}$ also resulted in a decrease of $\mathrm{CD} 8^{+} \mathrm{DC}$, which occurred with comparable kinetics to $\alpha$-GalCer. DC activation and depletion were tightly linked, with DC activation almost always correlating with a decline in the $\mathrm{CD} 8^{+} \mathrm{DC}$ subset. This loss has similar characteristics to activation induced cell death.

Treatment with low doses or $\alpha$-GalCer or anti-TNF- $\alpha$ reduced the loss of CD8 ${ }^{+} \mathrm{DC}$, but did not lead to significant improvements in $\mathrm{CD}^{+} \mathrm{T}$ cell responses to ova protein. This result is likely to be due to a reduced activation status of the DC that occurred during the process of retaining the number of $\mathrm{CD}^{+} \mathrm{DC}$. Improving responses would require that $\mathrm{DC}$ activation and loss be separated.

Taken together, these data provides further insights into the physiology of DC during the immune response to $\alpha$-GalCer and will hopefully help to improve future $\alpha$-GalCer based cancer immunotherapies. 
Chapter 6:

General Discussion 
The main aim of this thesis was to establish whether $\mathrm{CD}^{+} \mathrm{T}$ cells and NKT cells eliminate DC in vivo in a perforin-dependent manner, and whether this elimination results in reduced $\mathrm{T}$ cell responses due to limited antigen presentation.

At the time this study was initiated there was evidence indicating that CTL mediate DC killing in a perforin-dependent manner. However, this evidence arose from experiments that were conducted using immunised mice and BMDC loaded with peptide antigen (155). There are a number of limitations to this approach, such as the mice were injected with TCR transgenic T cells and the BMDC were loaded with a high concentration of a specific peptide, which is an artificial mode of antigen loading and presentation that does not reflect the physiological situation. Furthermore, the BMDC used in that study were an in vitro generated population that lacks an in vivo equivalent (155). Additionally, at the commencement of this study there was no in vivo data demonstrating whether NKT cells can eliminate DC. It was of interest to assess whether NKT cells have a role in DC killing as they express perforin and, like other perforin-expressing innate cells such as NK cells, may regulate DC survival and the outcome of immune responses (148). Therefore, two different experimental models were established to assess whether $\mathrm{CD}^{+} \mathrm{T}$ cell and NKT cells eliminate DC in vivo.

The first in vivo model established was a murine influenza A viral infection. As discussed in Chapter 3, this model was chosen because influenza infection elicits a robust specific $\mathrm{CD}^{+} \mathrm{T}$ cell response. Furthermore, as clearance of the influenza A virus does not require perforin (366), antigenic viral load in PKO mice is not expected to differ significantly from C57BL/6 mice. Among the drawbacks of this model are that, the antigen load is difficult to control experimentally, and that many CTL specific for numerous influenza antigens are present. To address some of these limitations, a second in vivo model was established which used immunisations with controlled amounts of antigen. The NKT cell ligand $\alpha$-GalCer and the protein antigen ova were administered to elicit NKT cell activation and an ova-specific CD8 ${ }^{+}$ $\mathrm{T}$ cell response, respectively, allowing the assessment of whether $\mathrm{CD} 8^{+} \mathrm{T}$ cells and NKT cells eliminated DC in a perforin-dependent manner. In both the influenza and the $\alpha$-GalCer model, DC are loaded with antigen in vivo, and endogenous $\mathrm{T}$ cell responses are assessed without the requirement for TCR transgenic CTL. The results of these studies indicated that during an 
influenza infection PKO mice did not generate an enhanced $\mathrm{CD}^{+} \mathrm{T}$ cell response compared to C57BL/6 mice. Furthermore, investigations in this thesis did not provide evidence for perforin-dependent elimination of DC by NKT cells, but instead revealed a loss of splenic $\mathrm{CD}^{+}$DC partly mediated by the cytokine TNF- $\alpha$.

Others have found that PKO mice generate enhanced immune responses after a range of infections $(338,339,374-378)$ leading to the proposal that increased DC survival was driving increased $\mathrm{T}$ cell expansion in these mice (155). Furthermore, increased $\mathrm{T}$ cell responses were observed in other systems where DC were genetically manipulated to prolong their survival (144, 157-162), providing additional support that increased DC survival correlates with an improved $\mathrm{T}$ cell response. The studies described in this thesis demonstrated that the primary $\mathrm{CD}^{+} \mathrm{T}$ cell response to influenza was not enhanced in PKO mice compared to C57BL/6 mice. These results are consistent with a published study showing that during a primary influenza infection the numbers of DC presenting influenza antigens were similar in the two mouse strains (156). Investigations into primary $\mathrm{CD}^{+} \mathrm{T}$ cell responses after treatment with $\alpha-$ GalCer and ova protein revealed similar T cell expansion in C57BL/6 and PKO mice, but were inconclusive as to whether ova-specific $\mathrm{CD}^{+} \mathrm{T}$ cells could mediate killing of splenic DC. Loss of splenic $\mathrm{CD} 8^{+} \mathrm{DC}$ was observed but appeared to be due to the administration of $\alpha-$ GalCer on NKT cells rather than the presence of ova-specific CTL, suggesting that the $\alpha$ GalCer model was not suitable for the study of CTL mediated DC elimination. Altogether, on the basis of these results it was not possible to conclude on a role of $\mathrm{T}$ cell derived perforin in regulating DC survival in vivo. It is likely that other experimental systems may be better suited to addressing this question.

As discussed in Chapter 1 , there is evidence suggesting that memory $\mathrm{CD} 8^{+} \mathrm{T}$ cells can kill DC, while naïve T cells are unable to do so. Thus secondary immune responses were induced to determine whether memory $\mathrm{CD} 8^{+} \mathrm{T}$ cells killed $\mathrm{DC}$, using the two model systems studied in this thesis. The advantage of the influenza A virus infection is that there is a well established method for eliciting a secondary influenza-specific immune response $(354,355,357)$. The results from these secondary influenza infection experiments revealed that, compared to C57BL/6 mice, $\mathrm{PKO}$ mice generated specific $\mathrm{CD} 8^{+} \mathrm{T}$ cell response that were not greater, or 
were even smaller, than the responses in C57BL/6 mice. This was surprising considering that data published during the course of this study showed that, during a secondary influenza infection, the numbers of DC presenting influenza antigen in the MLN are much larger in PKO mice than C57BL/6 mice (156). These data are at odds with the observation reported in this thesis that the $\mathrm{D}^{\mathrm{b}}-\mathrm{NP}_{366-374}{ }^{+} \mathrm{CD} 8^{+} \mathrm{T}$ cell response in peripheral blood was in fact reduced in PKO mice. Recent publications have reported that release of granzyme A by CTL induces the production of pro-inflammatory cytokines (444), which could lead to immunopathology. Granzyme A would be expected to be released in greater amounts in PKO mice, as a consequence of increased antigen presentation. This pro-inflammatory response could result in poor T cell proliferation and survival, and reduced expansion. Additionally, as a result of increased antigen presentation in PKO mice there is likely to be increased IFN- $\gamma$ production, which is important for antigen-specific $\mathrm{CD}^{+} \mathrm{T}$ cells homeostasis (338) and could also play a part in the decreased $\mathrm{T}$ cell response observed in PKO mice during a secondary influenza infection. To assess whether enhanced immunopathology or increased IFN- $\gamma$ plays a role in the reduced $\mathrm{T}$ cell response observed in PKO mice immunohistological staining of the lung tissue could be carried out and serum levels of IFN- $\gamma$ could be examined. Taken together, these data indicate that, during an influenza virus infection, increased antigen presentation by DC does not correlate with an enhanced influenza-specific $\mathrm{CD} 8^{+} \mathrm{T}$ cell response, and may even result in reduced responses possibly through increased inflammation and immunopathology.

Unfortunately, efforts to generate a secondary ova-specific $\mathrm{CD}^{+} \mathrm{T}$ cell response using the $\alpha$ GalCer and ova model were unsuccessful, and no information could be obtained on whether perforin regulates DC survival and T cell immune responses in this system.

Studies in PKO mice demonstrate that the perforin pathway is involved in defence against viral pathogens and transformed cells (236). Perforin was also shown to be important in controlling immune responses in several experimental systems $(339,375,378,445-447)$. In support of these studies, the human disorder Familial Hemophagocytic Lymphohistiocytosis (FHL), which is caused by mutations in perforin and the perforin/granule pathway $(341,448)$, is characterised by uncontrolled expansion of antigen-specific $\mathrm{CD} 8^{+} \mathrm{T}$ cells and macrophages, 
and a fatal outcome (341). An FHL-like syndrome is observed in PKO mice infected with LCMV (339). The lymphoproliferation observed in FHL patients and PKO mice infected with LCMV may be due to increased DC survival, resulting in continuous antigen presentation to T cells $(153,155,336)$. Hemophagocytic syndromes are associated with a viral trigger (449), and the requirement of perforin for clearance of LCMV raises the question of whether the observed lymphocyte expansion in FHL patients and PKO mice is due to an inability to clear the infectious agent itself. Support for this view comes from a study that used VSV, which does not require perforin for viral clearance, and found that C57BL/6 and PKO mice generated similar T cell responses (379), suggesting that enhanced immune responses observed by others (339) is due to the requirement for perforin in viral clearance. Similarly, the findings in this thesis and by others (372), demonstrated that PKO mice infected with influenza virus did not have enhanced $\mathrm{CD}^{+} \mathrm{T}$ cell responses compared to WT. Nevertheless, it seems that the lymphoproliferation observed in the absence of a functional perforin/granule pathway cannot simply be a consequence of increased viral load, as PKO mice injected with peptide antigen under limiting conditions also showed enhanced $\mathrm{T}$ cell responses $(155,378)$. These discrepancies in $\mathrm{T}$ cell expansion between model systems might be linked to defects in perforin expression in other cell populations such as T regulatory ( $\mathrm{T}$ reg) cells and NK cells. However, there is no evidence to indicate that defects in $\mathrm{T}$ reg cells affect $\mathrm{T}$ cell expansion in PKO mice injected with peptide antigen under limiting conditions (155), and it is not likely based on the similar rate of viral clearance between PKO and C57BL/6 mice that deficiency in perforin expression in either $\mathrm{T}$ reg or NK cells would affect immune responses during an influenza infection. Taken together there is evidence to suggest that perforin has an important role as an effector molecule in the clearance of certain viral infections, but as well there is data to suggest that perforin also has an immunoregulatory function. Thus, it is not yet fully understood the extent of the role perforin plays in immune responses. However, the ability of perforin to elicit its immunoregulatory function seems to depend on the nature of the stimulus, including pathogen virulence or strength of stimulation, and these requirements appear to differ between FHL patients and PKO mice. Though, the variation between FHL patients and PKO mice might reflect the clean environmental conditions the mice live in or be due to the immunologic differences between mouse and human. 
In this thesis, the loss of splenic $\mathrm{CD} 8^{+} \mathrm{DC}$ that was observed in the presence of activated NKT cells did not depend on perforin expression. This was in contrast to the in vitro assays that have demonstrated that human NKT cells can kill DC $(150,151,296,310)$ and tumour cell lines $(152,313-315,323)$ in a perforin-dependent manner $(310,312-315)$. However, perforin is differentially expressed in human NKT cell subsets with CD4 ${ }^{-}$NKT cells expressing higher levels of perforin than $\mathrm{CD}^{+}$NKT cells (235). CD4 ${ }^{-}$NKT cells are also less activated based on cell surface expression of CD69 and CD25 compared with CD4 ${ }^{+}$NKT cells (Nina Dickgreber, personal communication). These results are consistent with recent human studies demonstrating that the CD4 co-receptor is important for NKT cell activation and cytokine production $(450,451)$. Thus, these results suggest that NKT cells affect DC survival in vivo in a perforin-independent manner, which could be due to the differential expression of perforin in the NKT cell subsets.

Published evidence suggests that CTL can regulate DC survival by perforin $(155,156)$. However, CTL and NKT cells also express molecules such as FasL and TRAIL that are also involved in mediating death of target cells $(152,225,317,318,452)$. Recently, studies using mice that specifically lacked Fas expression on DC showed that Fas-FasL mediated killing of DC is an important regulatory mechanism to prevent systemic autoimmunity (453). This was consistent with a previous report showing that systemic autoimmunity develops in transgenic mice where DC accumulated due to a defect in apoptosis (164). In addition, it has been demonstrated that influenza-specific $\mathrm{CD} 8^{+} \mathrm{T}$ cells utilise TRAIL to mediate the death of influenza infected cells (454). DC killing was not directly assessed but it is possible that CTL could kill DC in a TRAIL specific manner. As well as other receptor-mediated cell death mechanisms, CTL and NKT cells also secrete the cytokine TNF- $\alpha$, which is known to induce cell death (427). Consistent with this, studies in this thesis revealed that in the presence of NKT cell activation, TNF- $\alpha$ mediated the selective loss of the CD ${ }^{+}$DC. However, although other mechanisms could be used to regulate DC survival, there is clear evidence that perforin plays an important role in DC survival during an influenza infection (156). Thus, DC can potentially be eliminated through multiple pathways but it appears that the predominant pathway used to eliminate DC varies between different immune responses. 
DC killing may be one active means of controlling and regulating the magnitude of immune responses, however, alternative mechanisms exist. T cells express CTLA-4 upon activation, which binds to CD80/CD86 on DC. Signalling through CTLA-4 blocks T cell proliferation and cytokine production $(109,112,117)$. Activation of T cells also leads to co-expression of the death receptor Fas and its ligand, FasL, resulting in activation induced cell death, via neighbouring T cells or DC (455). T reg cells may act directly on DC to prevent antigen presentation and cause incomplete activation of $\mathrm{T}$ cells. $\mathrm{T}$ reg cells may also be able to directly suppress T cell activity (456). Multiple T cell subsets can exert negative immunoregulatory functions by producing immunomodulatory cytokines such as IL-10 and TGF- $\beta$. In support of this, $\mathrm{CD}^{+}$and $\mathrm{CD} 8^{+} \mathrm{T}$ cells were recently shown to produce IL-10 during an influenza infection. IL-10 is thought to act directly on $\mathrm{CD}^{+}$and $\mathrm{CD} 8^{+} \mathrm{T}$ cells to dampen the immune response and reduce lung inflammation (457). Any of these mechanisms might be acting to prevent increased $\mathrm{T}$ cell proliferation in influenza infected PKO mice.

DC are being used as the basis of anti-cancer vaccines, however, after multiple clinical trials only a small number of positive clinical outcomes have been observed $(167,168,170)$. The aim of DC vaccine strategies is to improve anti-cancer $\mathrm{T}$ cell responses. As discussed in section 1.1.5.2, prolonging the survival of DC has been demonstrated to increase $\mathrm{T}$ cell responses $(144,157-162)$. However, extended DC survival has also been correlated with systemic autoimmunity $(163,164)$. Data in Chapter 3 also revealed that in situations where increased DC survival was reported (156) an enhanced T cell response does not always occur. This would suggest that ensuring the survival of the DC vaccine is important (170) but not enough to guarantee that the corresponding $\mathrm{T}$ cell response will be improved. Therefore, understanding which other mechanisms may be acting to limit the size of the $\mathrm{T}$ cell response will be an important goal of future studies. Together with an understanding of the mechanisms that regulate the size of the T cell response, other aspects of DC biology need to be the focus of DC vaccine research to enable the next generation of DC vaccines to be more effective. Results in chapter 4 and 5 indicate that DC subsets behave differently to certain stimuli, consequently it is likely that the DC subset used for anti-cancer vaccines is important. Furthermore, as mentioned in chapter 1, migration to the $\mathrm{LN}$ and the activation status of injected DC is critical to the generation of an effector $\mathrm{CD} 8^{+} \mathrm{T}$ cell response. Thus, with further 
research into these areas of DC biology it is hopeful that a successful DC vaccine will be produced in the near future.

In recent years the NKT cell ligand $\alpha$-GalCer has been used as an adjuvant in clinical trials in conjunction with DC vaccines as an anti-cancer treatment (458-462). Similar to DC vaccines without an adjuvant, the addition of $\alpha-\mathrm{GalCer}$ in the vaccine regime has generated few positive clinical outcomes (463). The DC-NKT cell interaction is central to the adjuvant effects of $\alpha$-GalCer. Studies in this thesis provide further insights into the physiology of DC during the immune response to $\alpha$-GalCer. It was found that after $\alpha$-GalCer treatment CD $8^{+}$ $\mathrm{DC}$, which are important for $\mathrm{CD} 8^{+} \mathrm{T}$ cell responses, were selectively lost. Nevertheless, loss of $\mathrm{CD}^{+} \mathrm{DC}$ did not prevent an antigen-specific $\mathrm{CD} 8^{+} \mathrm{T}$ cell response being generated. Thus, while the precise consequence of the loss of $\mathrm{CD}^{+} \mathrm{DC}$ requires further characterisation, this should not prevent the use of $\alpha$-GalCer as a powerful adjuvant for cancer immunotherapies.

Overall, it appears that multiple cell types, such as CTL and NKT cells, regulate the duration of antigen presentation, through several mechanisms including perforin- and cytokinedependent pathways. Depending on the model studied, the duration of antigen presentation may have a profound effect on the T cell response elicited. Furthermore, the studies conducted in this thesis highlight the need to use the appropriate in vivo experimental models to gain a greater understanding of the cell types that regulate DC survival and antigen presentation.

\subsection{Summary}

The major findings reported in this thesis can be summarised as follows:

- $\mathrm{C} 57 \mathrm{BL} / 6$ and PKO mice generated similar frequencies of antigen-specific CD8 ${ }^{+} \mathrm{T}$ cells during a primary influenza infection. 
- $\quad$ C57BL/6 and PKO mice generated different frequencies of antigen-specific CD8 ${ }^{+} \mathrm{T}$ cell during a secondary influenza infection.

- T cell exhaustion, evaluated as expression of the marker KLRG1, did not explain the diminished antigen-specific $\mathrm{CD} 8^{+} \mathrm{T}$ cell response in $\mathrm{PKO}$ mice during a secondary influenza infection.

- Treatment with the NKT cell ligands $\alpha-G a l C e r$ and OCH induced a transient loss of splenic $\mathrm{CD}^{+} \mathrm{DC}$, which was dose dependent and correlated with the induction of DC activation.

- The loss of $\mathrm{CD}^{+} \mathrm{DC}$ in $\alpha$-GalCer treated mice did not require expression of perforin, Fas or IFN- $\gamma$, but was prevented by treatment with an anti-TNF- $\alpha$ neutralising antibody, indicating that TNF- $\alpha$ played a role in $\mathrm{CD} 8^{+} \mathrm{DC}$ loss.

Taken together, these results suggest that increased DC survival does not necessarily correlate with an increased $\mathrm{T}$ cell response. Thus there appears to be a fine balance between DC survival, increased $\mathrm{T}$ cell responses and immunopathology with many factors influencing the ultimate outcome of the host immune response.

In addition, this thesis also showed that NKT cells play a role in the regulation of DC survival in vivo, which is at least in part mediated by the production of the cytokine TNF- $\alpha$. Furthermore, the different splenic DC subsets are differentially regulated with the CD8 ${ }^{+} \mathrm{DC}$ being more susceptible to NKT cell mediated reduction than the CD8 ${ }^{-} \mathrm{DC}$ subset. These findings provide additional knowledge about the regulation of DC survival and will hopefully assist in improving DC vaccines in the future. 


\subsection{Future Directions}

The work described in this thesis has raised a number of questions that will require detailed experimentation to be resolved.

The experiments in Chapter 3 revealed that during a secondary influenza infection PKO mice had a significantly reduced $\mathrm{CD} 8^{+} \mathrm{D}^{\mathrm{b}}-\mathrm{NP}_{366-374}{ }^{+} \mathrm{T}$ cell response compared to $\mathrm{C} 57 \mathrm{BL} / 6$ mice. This result was inconsistent with the increased DC survival and hence antigen presentation observed by others (156). Expression of KLRG1 was investigated to assess whether the T cells in the PKO mice were exhausted and terminally differentiated, but no significant difference was found between PKO and C57BL/6 mice. As well as KLRG1 there are many other markers that have been associated with $\mathrm{T}$ cell exhaustion and a terminally differentiated phenotype, such as PD1 (385). Therefore investigation into the expression level of other markers on influenza-specific $\mathrm{CD}^{+}{ }^{+} \mathrm{T}$ cells in C57BL/6 and PKO mice may reveal phenotypic differences that affect the functional capacity of the cells, possibly accounting for the observed difference in the expansion of influenza-specific $\mathrm{CD}^{+} \mathrm{T}$ cells in $\mathrm{C} 57 \mathrm{BL} / 6$ and $\mathrm{PKO}$ mice during a secondary influenza infection.

$\mathrm{CD}^{+} \mathrm{DC}$ appear to have a specialised role in viral immunity (416) and are the only DC subset that can induce the proliferation of memory $\mathrm{CD}^{+} \mathrm{T}$ cells during an influenza infection (464). Consequently, selective elimination of $\mathrm{CD}^{+} \mathrm{DC}$ during infections could be a mechanism to regulate memory $\mathrm{T}$ cells expansion thereby reducing immunopathology and limiting $\mathrm{T}$ cell expansion. It would therefore be of interest to determine if memory influenza-specific $\mathrm{CD} 8^{+} \mathrm{T}$ cells, which have been shown to eliminate DC (156), selectively eliminate a specific DC subset in the influenza model. These experiments would provide additional insights into DC elimination by memory $\mathrm{CD}^{+} \mathrm{T}$ cells as a mechanism of immune regulation.

During this study the generation of a secondary ova-specific $\mathrm{CD} 8^{+} \mathrm{T}$ cell response was attempted, to address the question of whether CTL kill DC in a non-infectious setting. Unfortunately a secondary ova-specific $\mathrm{CD} 8^{+} \mathrm{T}$ cell could not be successfully generated using the experimental model chosen. It would be of interest to investigate this question using an different method. Another model system shown to generate an effective secondary ova- 
specific $\mathrm{CD}^{+} \mathrm{T}$ cell response, is the use of ova protein followed by recombinant vaccinia virus expressing full-length ova protein. Experiments using this model to assess a secondary ova-specific $\mathrm{CD}^{+} \mathrm{T}$ cell response could provide information on the role of CTL elimination of DC other than in the murine influenza A virus model used here.

A possible avenue for future research is to elucidate the role membrane bound TNF in the decline of $\mathrm{CD}^{+} \mathrm{DC}$ after treatment with $\alpha$-GalCer. As discussed in Chapter 5, mTNF has cytotoxic effects and could also be involved in the loss of the CD8 ${ }^{+} \mathrm{DC}$ after $\alpha$-GalCer treatment. To evaluate the contribution of mTNF and sTNF in the loss of CD8 ${ }^{+}$DC after $\alpha-$ GalCer treatment, knockout mice of each form of TNF could be used. Furthermore, it would be of interest to determine the cellular source of the TNF that mediates the loss of $\mathrm{CD} 8^{+} \mathrm{DC}$, and to assess if $\mathrm{CD}^{+} \mathrm{DC}$ express higher levels of TNFR compared to CD8 ${ }^{-} \mathrm{DC}$, resulting in increased sensitivity to TNF- $\alpha$ after $\alpha$-GalCer treatment. These results would provide additional information about the mechanism of the loss of $\mathrm{CD} 8^{+} \mathrm{DC}$ after $\alpha$-GalCer treatment. 


\section{References}

1. Banchereau, J., and R. M. Steinman. 1998. Dendritic cells and the control of immunity. Nature 392:245-252.

2. Steinman, R. M., D. S. Lustig, and Z. A. Cohn. 1974. Identification of a novel cell type in peripheral lymphoid organs of mice. 3. Functional properties in vivo. $J$ Exp Med 139:1431-1445.

3. Akashi, K., D. Traver, T. Miyamoto, and I. L. Weissman. 2000. A clonogenic common myeloid progenitor that gives rise to all myeloid lineages. Nature 404:193-197.

4. Kondo, M., I. L. Weissman, and K. Akashi. 1997. Identification of clonogenic common lymphoid progenitors in mouse bone marrow. Cell 91:661-672.

5. Manz, M. G., D. Traver, T. Miyamoto, I. L. Weissman, and K. Akashi. 2001. Dendritic cell potentials of early lymphoid and myeloid progenitors. Blood 97:3333-3341.

6. Traver, D., K. Akashi, M. G. Manz, M. Merad, T. Miyamoto, E. G. Engleman, and I. L. Weissman. 2000. Development of CD8alpha-positive dendritic cells from a common myeloid progenitor. Science 290:2152-2154.

7. Wu, L., A. D'Amico, H. Hochrein, M. O'Keeffe, K. Shortman, and K. Lucas. 2001. Development of thymic and splenic dendritic cell populations from different hemopoietic precursors. Blood 98:3376-3382.

8. Gilliland, D. G., and J. D. Griffin. 2002. The roles of FLT3 in hematopoiesis and leukemia. Blood 100:1532-1542.

9. D'Amico, A., and L. Wu. 2003. The early progenitors of mouse dendritic cells and plasmacytoid predendritic cells are within the bone marrow hemopoietic precursors expressing Flt3. J Exp Med 198:293-303.

10. Karsunky, H., M. Merad, A. Cozzio, I. L. Weissman, and M. G. Manz. 2003. Flt3 ligand regulates dendritic cell development from Flt3+ lymphoid and myeloidcommitted progenitors to Flt3+ dendritic cells in vivo. J Exp Med 198:305-313.

11. Hannum, C., J. Culpepper, D. Campbell, T. McClanahan, S. Zurawski, J. F. Bazan, R. Kastelein, S. Hudak, J. Wagner, and J. Mattson. 1994. Ligand for FLT3/FLK2 receptor tyrosine kinase regulates growth of haematopoietic stem cells and is encoded by variant RNAs. Nature 368:643-648.

12. Lyman, S. D., L. James, T. Vanden Bos, P. de Vries, K. Brasel, B. Gliniak, L. T. Hollingsworth, K. S. Picha, H. J. McKenna, and R. R. Splett. 1993. Molecular cloning of a ligand for the flt3/flk-2 tyrosine kinase receptor: a proliferative factor for primitive hematopoietic cells. Cell 75:1157-1167.

13. Shortman, K., and S. Naik. 2007. Steady-state and inflammatory dendritic-cell development. Nat Rev Immunol 7:19-30.

14. Inaba, K., M. Inaba, M. Deguchi, K. Hagi, R. Yasumizu, S. Ikehara, S. Muramatsu, and R. M. Steinman. 1993. Granulocytes, macrophages, and dendritic cells arise from a common major histocompatibility complex class II-negative progenitor in mouse bone marrow. Proc Natl Acad Sci USA 90:3038-3042.

15. Caux, C., B. Vanbervliet, C. Massacrier, C. Dezutter-Dambuyant, B. de Saint-Vis, C. Jacquet, K. Yoneda, S. Imamura, D. Schmitt, and J. Banchereau. 1996. CD34+ hematopoietic progenitors from human cord blood differentiate along two independent dendritic cell pathways in response to GM-CSF+TNF alpha. J Exp Med 184:695-706. 
16. Reid, C. D., A. Stackpoole, A. Meager, and J. Tikerpae. 1992. Interactions of tumor necrosis factor with granulocyte-macrophage colony-stimulating factor and other cytokines in the regulation of dendritic cell growth in vitro from early bipotent CD34+ progenitors in human bone marrow. J Immunol 149:2681-2688.

17. Szabolcs, P., D. Avigan, S. Gezelter, D. H. Ciocon, M. A. Moore, R. M. Steinman, and J. W. Young. 1996. Dendritic cells and macrophages can mature independently from a human bone marrow-derived, post-colony-forming unit intermediate. Blood 87:45204530.

18. Manz, M. G., D. Traver, K. Akashi, M. Merad, T. Miyamoto, E. G. Engleman, and I. L. Weissman. 2001. Dendritic cell development from common myeloid progenitors. Ann N Y Acad Sci 938:167-173.

19. Vremec, D., M. Zorbas, R. Scollay, D. J. Saunders, C. F. Ardavin, L. Wu, and K. Shortman. 1992. The surface phenotype of dendritic cells purified from mouse thymus and spleen: investigation of the CD8 expression by a subpopulation of dendritic cells. $J$ Exp Med 176:47-58.

20. Ardavin, C. F., L. Wu, C. L. Li, and K. Shortman. 1993. Thymic dendritic cells and T cells develop simultaneously in the thymus from a common precursor population. Nature 362:761-763.

21. Bendriss-Vermare, N., C. Barthélémy, I. Durand, C. Bruand, C. Dezutter-Dambuyant, N. Moulian, S. Berrih-Aknin, C. Caux, G. Trinchieri, and F. Brière. 2001. Human thymus contains IFN-alpha-producing CD11c(-), myeloid CD11c(+), and mature interdigitating dendritic cells. J Clin Invest 107:835-844.

22. Res, P. C., F. Couwenberg, F. A. Vyth-Dreese, and H. Spits. 1999. Expression of pTalpha mRNA in a committed dendritic cell precursor in the human thymus. Blood 94:2647-2657.

23. Corcoran, L., I. Ferrero, D. Vremec, K. Lucas, J. Waithman, M. A. O'Keeffe, L. Wu, A. Wilson, and K. Shortman. 2003. The lymphoid past of mouse plasmacytoid cells and thymic dendritic cells. J Immunol 170:4926-4932.

24. Shigematsu, H., B. Reizis, H. Iwasaki, S. Mizuno, D. Hu, D. Traver, P. Leder, N. Sakaguchi, and K. Akashi. 2004. Plasmacytoid dendritic cells activate lymphoidspecific genetic programs irrespective of their cellular origin. Immunity 21:43-53.

25. Spits, H., F. Couwenberg, A. Q. Bakker, K. Weijer, and C. H. Uittenbogaart. 2000. Id2 and Id3 inhibit development of CD34(+) stem cells into predendritic cell (pre-DC)2 but not into pre-DC1. Evidence for a lymphoid origin of pre-DC2. J Exp Med 192:1775-1784.

26. Chicha, L., D. Jarrossay, and M. G. Manz. 2004. Clonal type I interferon-producing and dendritic cell precursors are contained in both human lymphoid and myeloid progenitor populations. J Exp Med 200:1519-1524.

27. Jiang, W., W. J. Swiggard, C. Heufler, M. Peng, A. Mirza, R. M. Steinman, and M. C. Nussenzweig. 1995. The receptor DEC-205 expressed by dendritic cells and thymic epithelial cells is involved in antigen processing. Nature 375:151-155.

28. Sallusto, F., M. Cella, C. Danieli, and A. Lanzavecchia. 1995. Dendritic cells use macropinocytosis and the mannose receptor to concentrate macromolecules in the major histocompatibility complex class II compartment: downregulation by cytokines and bacterial products. J Exp Med 182:389-400.

29. Reis e Sousa, C., P. D. Stahl, and J. M. Austyn. 1993. Phagocytosis of antigens by Langerhans cells in vitro. J Exp Med 178:509-519. 
30. Regnault, A., D. Lankar, V. Lacabanne, A. Rodriguez, C. Théry, M. Rescigno, T. Saito, S. Verbeek, C. Bonnerot, P. Ricciardi-Castagnoli, and S. Amigorena. 1999. Fcgamma receptor-mediated induction of dendritic cell maturation and major histocompatibility complex class I-restricted antigen presentation after immune complex internalization. J Exp Med 189:371-380.

31. Reis E Sousa, C. 2006. Dendritic cells in a mature age. Nat Rev Immunol 6:476-483.

32. Liu, K., T. Iyoda, M. Saternus, Y. Kimura, K. Inaba, and R. M. Steinman. 2002. Immune tolerance after delivery of dying cells to dendritic cells in situ. $J$ Exp Med 196:1091-1097.

33. Hernandez, J., S. Aung, W. L. Redmond, and L. A. Sherman. 2001. Phenotypic and functional analysis of $\mathrm{CD} 8(+) \mathrm{T}$ cells undergoing peripheral deletion in response to cross-presentation of self-antigen. J Exp Med 194:707-717.

34. Belz, G. T., G. M. Behrens, C. M. Smith, J. F. Miller, C. Jones, K. Lejon, C. G. Fathman, S. N. Mueller, K. Shortman, F. R. Carbone, and W. R. Heath. 2002. The CD8alpha(+) dendritic cell is responsible for inducing peripheral self-tolerance to tissue-associated antigens. J Exp Med 196:1099-1104.

35. Hawiger, D., K. Inaba, Y. Dorsett, M. Guo, K. Mahnke, M. Rivera, J. V. Ravetch, R. M. Steinman, and M. C. Nussenzweig. 2001. Dendritic cells induce peripheral T cell unresponsiveness under steady state conditions in vivo. J Exp Med 194:769-779.

36. Bonifaz, L., D. Bonnyay, K. Mahnke, M. Rivera, M. C. Nussenzweig, and R. M. Steinman. 2002. Efficient targeting of protein antigen to the dendritic cell receptor DEC-205 in the steady state leads to antigen presentation on major histocompatibility complex class I products and peripheral CD8+ T cell tolerance. J Exp Med 196:16271638.

37. Steinman, R. M., and M. C. Nussenzweig. 2002. Avoiding horror autotoxicus: the importance of dendritic cells in peripheral T cell tolerance. Proc Natl Acad Sci USA 99:351-358.

38. Akira, S., K. Takeda, and T. Kaisho. 2001. Toll-like receptors: critical proteins linking innate and acquired immunity. Nat Immunol 2:675-680.

39. Kaisho, T., and S. Akira. 2001. Dendritic-cell function in Toll-like receptor- and MyD88-knockout mice. Trends Immunol 22:78-83.

40. Cella, M., A. Engering, V. Pinet, J. Pieters, and A. Lanzavecchia. 1997. Inflammatory stimuli induce accumulation of MHC class II complexes on dendritic cells. Nature 388:782-787.

41. Sallusto, F., P. Schaerli, P. Loetscher, C. Schaniel, D. Lenig, C. R. Mackay, S. Qin, and A. Lanzavecchia. 1998. Rapid and coordinated switch in chemokine receptor expression during dendritic cell maturation. Eur J Immunol 28:2760-2769.

42. Cella, M., D. Scheidegger, K. Palmer-Lehmann, P. Lane, A. Lanzavecchia, and G. Alber. 1996. Ligation of CD40 on dendritic cells triggers production of high levels of interleukin-12 and enhances T cell stimulatory capacity: T-T help via APC activation. J Exp Med 184:747-752.

43. Koch, F., U. Stanzl, P. Jennewein, K. Janke, C. Heufler, E. Kämpgen, N. Romani, and G. Schuler. 1996. High level IL-12 production by murine dendritic cells: upregulation via MHC class II and CD40 molecules and downregulation by IL-4 and IL-10. J Exp Med 184:741-746.

44. Reis e Sousa, C., S. Hieny, T. Scharton-Kersten, D. Jankovic, H. Charest, R. N. Germain, and A. Sher. 1997. In vivo microbial stimulation induces rapid CD40 ligand- 
independent production of interleukin 12 by dendritic cells and their redistribution to $\mathrm{T}$ cell areas. J Exp Med 186:1819-1829.

45. Guermonprez, P., J. Valladeau, L. Zitvogel, C. Théry, and S. Amigorena. 2002. Antigen presentation and T cell stimulation by dendritic cells. Annu Rev Immunol 20:621-667.

46. Shortman, K., and Y. J. Liu. 2002. Mouse and human dendritic cell subtypes. Nat Rev Immunol 2:151-161.

47. Colonna, M., G. Trinchieri, and Y. J. Liu. 2004. Plasmacytoid dendritic cells in immunity. Nat Immunol 5:1219-1226.

48. Liu, Y. J. 2005. IPC: professional type 1 interferon-producing cells and plasmacytoid dendritic cell precursors. Annu Rev Immunol 23:275-306.

49. Iwasaki, A., and R. Medzhitov. 2004. Toll-like receptor control of the adaptive immune responses. Nat Immunol 5:987-995.

50. Grouard, G., M. C. Rissoan, L. Filgueira, I. Durand, J. Banchereau, and Y. J. Liu. 1997. The enigmatic plasmacytoid T cells develop into dendritic cells with interleukin (IL)-3 and CD40-ligand. J Exp Med 185:1101-1111.

51. Henri, S., D. Vremec, A. T. Kamath, J. Waithman, S. Williams, C. Benoist, K. Burnham, S. Saeland, E. Handman, and K. Shortman. 2001. The dendritic cell populations of mouse lymph nodes. J Immunol 167:741-748.

52. Valladeau, J., O. Ravel, C. Dezutter-Dambuyant, K. Moore, M. Kleijmeer, Y. Liu, V. Duvert-Frances, C. Vincent, D. Schmitt, J. Davoust, C. Caux, S. Lebecque, and S. Saeland. 2000. Langerin, a novel C-type lectin specific to Langerhans cells, is an endocytic receptor that induces the formation of Birbeck granules. Immunity 12:71-81.

53. Bursch, L. S., L. Wang, B. Igyarto, A. Kissenpfennig, B. Malissen, D. H. Kaplan, and K. A. Hogquist. 2007. Identification of a novel population of Langerin+ dendritic cells. J Exp Med 204:3147-3156.

54. Ginhoux, F., M. P. Collin, M. Bogunovic, M. Abel, M. Leboeuf, J. Helft, J. Ochando, A. Kissenpfennig, B. Malissen, M. Grisotto, H. Snoeck, G. Randolph, and M. Merad. 2007. Blood-derived dermal langerin+ dendritic cells survey the skin in the steady state. J Exp Med 204:3133-3146.

55. Poulin, L. F., S. Henri, B. de Bovis, E. Devilard, A. Kissenpfennig, and B. Malissen. 2007. The dermis contains langerin+ dendritic cells that develop and function independently of epidermal Langerhans cells. J Exp Med 204:3119-3131.

56. Merad, M., F. Ginhoux, and M. Collin. 2008. Origin, homeostasis and function of Langerhans cells and other langerin-expressing dendritic cells. Nat Rev Immunol 8:935-947.

57. Kissenpfennig, A., S. Henri, B. Dubois, C. Laplace-Builhé, P. Perrin, N. Romani, C. H. Tripp, P. Douillard, L. Leserman, D. Kaiserlian, S. Saeland, J. Davoust, and B. Malissen. 2005. Dynamics and function of Langerhans cells in vivo: dermal dendritic cells colonize lymph node areas distinct from slower migrating Langerhans cells. Immunity 22:643-654.

58. Vremec, D., J. Pooley, H. Hochrein, L. Wu, and K. Shortman. 2000. CD4 and CD8 expression by dendritic cell subtypes in mouse thymus and spleen. $J$ Immunol 164:2978-2986.

59. Vremec, D., and K. Shortman. 1997. Dendritic cell subtypes in mouse lymphoid organs: cross-correlation of surface markers, changes with incubation, and differences among thymus, spleen, and lymph nodes. J Immunol 159:565-573. 
60. Villadangos, J. A., and P. Schnorrer. 2007. Intrinsic and cooperative antigenpresenting functions of dendritic-cell subsets in vivo. Nat Rev Immunol 7:543-555.

61. De Smedt, T., B. Pajak, E. Muraille, L. Lespagnard, E. Heinen, P. De Baetselier, J. Urbain, O. Leo, and M. Moser. 1996. Regulation of dendritic cell numbers and maturation by lipopolysaccharide in vivo. J Exp Med 184:1413-1424.

62. Pulendran, B., J. Lingappa, M. K. Kennedy, J. Smith, M. Teepe, A. Rudensky, C. R. Maliszewski, and E. Maraskovsky. 1997. Developmental pathways of dendritic cells in vivo: distinct function, phenotype, and localization of dendritic cell subsets in FLT3 ligand-treated mice. J Immunol 159:2222-2231.

63. Hochrein, H., K. Shortman, D. Vremec, B. Scott, P. Hertzog, and M. O'Keeffe. 2001. Differential production of IL-12, IFN-alpha, and IFN-gamma by mouse dendritic cell subsets. J Immunol 166:5448-5455.

64. Edwards, A. D., D. Chaussabel, S. Tomlinson, O. Schulz, A. Sher, and C. Reis E Sousa. 2003. Relationships among murine CD11c(high) dendritic cell subsets as revealed by baseline gene expression patterns. J Immunol 171:47-60.

65. Edwards, A. D., S. Diebold, E. M. Slack, H. Tomizawa, H. Hemmi, T. Kaisho, S. Akira, and C. Reis E Sousa. 2003. Toll-like receptor expression in murine DC subsets: lack of TLR7 expression by CD8 alpha+ DC correlates with unresponsiveness to imidazoquinolines. Eur J Immunol 33:827-833.

66. Schulz, O., S. Diebold, M. Chen, T. I. Näslund, M. A. Nolte, L. Alexopoulou, Y. T. Azuma, R. Flavell, P. Liljeström, and C. Reis E Sousa. 2005. Toll-like receptor 3 promotes cross-priming to virus-infected cells. Nature 433:887-892.

67. Doxsee, C. L., T. R. Riter, M. J. Reiter, S. J. Gibson, J. P. Vasilakos, and R. M. Kedl. 2003. The immune response modifier and Toll-like receptor 7 agonist S-27609 selectively induces IL-12 and TNF-alpha production in CD11c+CD11b+CD8dendritic cells. J Immunol 171:1156-1163.

68. Dieu, M. C., B. Vanbervliet, A. Vicari, J. M. Bridon, E. Oldham, S. Aït-Yahia, F. Brière, A. Zlotnik, S. Lebecque, and C. Caux. 1998. Selective recruitment of immature and mature dendritic cells by distinct chemokines expressed in different anatomic sites. J Exp Med 188:373-386.

69. Sozzani, S., P. Allavena, G. D'Amico, W. Luini, G. Bianchi, M. Kataura, T. Imai, O. Yoshie, R. Bonecchi, and A. Mantovani. 1998. Differential regulation of chemokine receptors during dendritic cell maturation: a model for their trafficking properties. $J$ Immunol 161:1083-1086.

70. Yanagihara, S., E. Komura, J. Nagafune, H. Watarai, and Y. Yamaguchi. 1998. EBI1/CCR7 is a new member of dendritic cell chemokine receptor that is up-regulated upon maturation. J Immunol 161:3096-3102.

71. Verbovetski, I., H. Bychkov, U. Trahtemberg, I. Shapira, M. Hareuveni, O. Ben-Tal, I. Kutikov, O. Gill, and D. Mevorach. 2002. Opsonization of apoptotic cells by autologous iC3b facilitates clearance by immature dendritic cells, down-regulates DR and CD86, and up-regulates CC chemokine receptor 7. J Exp Med 196:1553-1561.

72. Ohl, L., M. Mohaupt, N. Czeloth, G. Hintzen, Z. Kiafard, J. Zwirner, T. Blankenstein, G. Henning, and R. Förster. 2004. CCR7 governs skin dendritic cell migration under inflammatory and steady-state conditions. Immunity 21:279-288.

73. Martín-Fontecha, A., S. Sebastiani, U. E. Höpken, M. Uguccioni, M. Lipp, A. Lanzavecchia, and F. Sallusto. 2003. Regulation of dendritic cell migration to the 
draining lymph node: impact on T lymphocyte traffic and priming. $J$ Exp Med 198:615-621.

74. Randolph, G., V. Angeli, and M. Swartz. 2005. Dendritic-cell trafficking to lymph nodes through lymphatic vessels. Nat Rev Immunol 5:617-628.

75. Sánchez-Sánchez, N., L. Riol-Blanco, and J. L. Rodríguez-Fernández. 2006. The multiple personalities of the chemokine receptor CCR7 in dendritic cells. J Immunol 176:5153-5159.

76. Kabashima, K., N. Shiraishi, K. Sugita, T. Mori, A. Onoue, M. Kobayashi, J. Sakabe, R. Yoshiki, H. Tamamura, N. Fujii, K. Inaba, and Y. Tokura. 2007. CXCL12-CXCR4 engagement is required for migration of cutaneous dendritic cells. Am J Pathol 171:1249-1257.

77. Willimann, K., D. F. Legler, M. Loetscher, R. S. Roos, M. B. Delgado, I. Clark-Lewis, M. Baggiolini, and B. Moser. 1998. The chemokine SLC is expressed in T cell areas of lymph nodes and mucosal lymphoid tissues and attracts activated T cells via CCR7. Eur J Immunol 28:2025-2034.

78. Ngo, V. N., H. L. Tang, and J. G. Cyster. 1998. Epstein-Barr virus-induced molecule 1 ligand chemokine is expressed by dendritic cells in lymphoid tissues and strongly attracts naive T cells and activated B cells. J Exp Med 188:181-191.

79. Kriehuber, E., S. Breiteneder-Geleff, M. Groeger, A. Soleiman, S. F. Schoppmann, G. Stingl, D. Kerjaschki, and D. Maurer. 2001. Isolation and characterization of dermal lymphatic and blood endothelial cells reveal stable and functionally specialized cell lineages. J Exp Med 194:797-808.

80. Gunn, M. D., K. Tangemann, C. Tam, J. G. Cyster, S. D. Rosen, and L. T. Williams. 1998. A chemokine expressed in lymphoid high endothelial venules promotes the adhesion and chemotaxis of naive T lymphocytes. Proc Natl Acad Sci USA 95:258263.

81. Kamath, A. T., S. Henri, F. Battye, D. F. Tough, and K. Shortman. 2002. Developmental kinetics and lifespan of dendritic cells in mouse lymphoid organs. Blood 100:1734-1741.

82. Randolph, G., J. Ochando, and S. Partida-Sánchez. 2008. Migration of dendritic cell subsets and their precursors. Annu Rev Immunol 26:293-316.

83. Liu, K., G. D. Victora, T. A. Schwickert, P. Guermonprez, M. M. Meredith, K. Yao, F. F. Chu, G. Randolph, A. Y. Rudensky, and M. Nussenzweig. 2009. In vivo analysis of dendritic cell development and homeostasis. Science 324:392-397.

84. Cella, M., D. Jarrossay, F. Facchetti, O. Alebardi, H. Nakajima, A. Lanzavecchia, and M. Colonna. 1999. Plasmacytoid monocytes migrate to inflamed lymph nodes and produce large amounts of type I interferon. Nat Med 5:919-923.

85. Diacovo, T. G., A. L. Blasius, T. W. Mak, M. Cella, and M. Colonna. 2005. Adhesive mechanisms governing interferon-producing cell recruitment into lymph nodes. $J$ Exp Med 202:687-696.

86. Mach, B., V. Steimle, E. Martinez-Soria, and W. Reith. 1996. Regulation of MHC class II genes: lessons from a disease. Annu Rev Immunol 14:301-331.

87. Chtanova, T., S. G. Tangye, R. Newton, N. Frank, M. R. Hodge, M. S. Rolph, and C. R. Mackay. 2004. T follicular helper cells express a distinctive transcriptional profile, reflecting their role as non-Th1/Th2 effector cells that provide help for B cells. $J$ Immunol 173:68-78. 
88. Nurieva, R. I., Y. Chung, D. Hwang, X. O. Yang, H. S. Kang, L. Ma, Y.-h. Wang, S. S. Watowich, A. M. Jetten, Q. Tian, and C. Dong. 2008. Generation of T follicular helper cells is mediated by interleukin-21 but independent of T helper 1,2, or 17 cell lineages. Immunity 29:138-149.

89. Zhu, J., and W. E. Paul. 2008. CD4 T cells: fates, functions, and faults. Blood 112:1557-1569.

90. Banchereau, J., F. Briere, C. Caux, J. Davoust, S. Lebecque, Y. J. Liu, B. Pulendran, and K. A. Palucka. 2000. Immunobiology of dendritic cells. Annu Rev Immunol 18:767-811.

91. Brigl, M., and M. B. Brenner. 2004. CD1: antigen presentation and T cell function. Annu Rev Immunol 22:817-890.

92. Brossay, L., D. Jullien, S. Cardell, B. C. Sydora, N. Burdin, R. L. Modlin, and M. Kronenberg. 1997. Mouse CD1 is mainly expressed on hemopoietic-derived cells. $J$ Immunol 159:1216-1224.

93. Roark, J. H., S. H. Park, J. Jayawardena, U. Kavita, M. Shannon, and A. Bendelac. 1998. CD1.1 expression by mouse antigen-presenting cells and marginal zone B cells. J Immunol 160:3121-3127.

94. Zeng, Z., A. R. Castaño, B. W. Segelke, E. A. Stura, P. A. Peterson, and I. A. Wilson. 1997. Crystal structure of mouse CD1: An MHC-like fold with a large hydrophobic binding groove. Science 277:339-345.

95. Beckman, E. M., S. A. Porcelli, C. T. Morita, S. M. Behar, S. T. Furlong, and M. B. Brenner. 1994. Recognition of a lipid antigen by CD1-restricted alpha beta+ T cells. Nature 372:691-694.

96. Bevan, M. J. 1976. Cross-priming for a secondary cytotoxic response to minor $\mathrm{H}$ antigens with $\mathrm{H}-2$ congenic cells which do not cross-react in the cytotoxic assay. $J$ Exp Med 143:1283-1288.

97. Lin, M. L., Y. Zhan, J. A. Villadangos, and A. M. Lew. 2008. The cell biology of cross-presentation and the role of dendritic cell subsets. Immunol Cell Biol 86:353-362.

98. Heath, W. R., G. T. Belz, G. M. Behrens, C. M. Smith, S. P. Forehan, I. A. Parish, G. M. Davey, N. Wilson, F. R. Carbone, and J. A. Villadangos. 2004. Cross-presentation, dendritic cell subsets, and the generation of immunity to cellular antigens. Immunol Rev 199:9-26.

99. Jung, S., D. Unutmaz, P. Wong, G. Sano, K. De los Santos, T. Sparwasser, S. Wu, S. Vuthoori, K. Ko, F. Zavala, E. G. Pamer, D. R. Littman, and R. A. Lang. 2002. In vivo depletion of CD11c(+) dendritic cells abrogates priming of CD8(+) T cells by exogenous cell-associated antigens. Immunity 17:211-220.

100. den Haan, J. M., S. M. Lehar, and M. J. Bevan. 2000. CD8(+) but not CD8(-) dendritic cells cross-prime cytotoxic T cells in vivo. J Exp Med 192:1685-1696.

101. Pooley, J. L., W. R. Heath, and K. Shortman. 2001. Cutting edge: intravenous soluble antigen is presented to CD4 T cells by CD8- dendritic cells, but cross-presented to CD8 T cells by CD8+ dendritic cells. J Immunol 166:5327-5330.

102. Schnorrer, P., G. M. Behrens, N. Wilson, J. L. Pooley, C. M. Smith, D. El-Sukkari, G. Davey, F. Kupresanin, M. Li, E. Maraskovsky, G. T. Belz, F. R. Carbone, K. Shortman, W. R. Heath, and J. A. Villadangos. 2006. The dominant role of CD8+ dendritic cells in cross-presentation is not dictated by antigen capture. Proc Natl Acad Sci USA 103:10729-10734. 
103. Carter, R. W., C. Thompson, D. M. Reid, S. Y. Wong, and D. F. Tough. 2006. Preferential induction of CD4+ T cell responses through in vivo targeting of antigen to dendritic cell-associated C-type lectin-1. J Immunol 177:2276-2284.

104. Dudziak, D., A. O. Kamphorst, G. F. Heidkamp, V. R. Buchholz, C. Trumpfheller, S. Yamazaki, C. Cheong, K. Liu, H. W. Lee, C. G. Park, R. M. Steinman, and M. C. Nussenzweig. 2007. Differential antigen processing by dendritic cell subsets in vivo. Science 315:107-111.

105. Burgdorf, S., A. Kautz, V. Böhnert, P. A. Knolle, and C. Kurts. 2007. Distinct pathways of antigen uptake and intracellular routing in CD4 and CD8 T cell activation. Science 316:612-616.

106. Acuto, O., and F. Michel. 2003. CD28-mediated co-stimulation: a quantitative support for TCR signalling. Nat Rev Immunol 3:939-951.

107. Gross, J. A., E. Callas, and J. P. Allison. 1992. Identification and distribution of the costimulatory receptor CD28 in the mouse. J Immunol 149:380-388.

108. Boise, L. H., A. J. Minn, P. J. Noel, C. H. June, M. A. Accavitti, T. Lindsten, and C. B. Thompson. 1995. CD28 costimulation can promote $\mathrm{T}$ cell survival by enhancing the expression of Bcl-XL. Immunity 3:87-98.

109. Lenschow, D. J., T. L. Walunas, and J. A. Bluestone. 1996. CD28/B7 system of T cell costimulation. Annu Rev Immunol 14:233-258.

110. McAdam, A. J., A. N. Schweitzer, and A. H. Sharpe. 1998. The role of B7 costimulation in activation and differentiation of CD4+ and CD8+ T cells. Immunol Rev 165:231-247.

111. Viola, A., S. Schroeder, Y. Sakakibara, and A. Lanzavecchia. 1999. T lymphocyte costimulation mediated by reorganization of membrane microdomains. Science 283:680-682.

112. Linsley, P. S., W. Brady, M. Urnes, L. S. Grosmaire, N. K. Damle, and J. A. Ledbetter. 1991. CTLA-4 is a second receptor for the B cell activation antigen B7. J Exp Med 174:561-569.

113. Linsley, P. S., J. Bradshaw, J. Greene, R. Peach, K. L. Bennett, and R. S. Mittler. 1996. Intracellular trafficking of CTLA-4 and focal localization towards sites of TCR engagement. Immunity 4:535-543.

114. Linsley, P. S., J. L. Greene, P. Tan, J. Bradshaw, J. A. Ledbetter, C. Anasetti, and N. K. Damle. 1992. Coexpression and functional cooperation of CTLA-4 and CD28 on activated T lymphocytes. J Exp Med 176:1595-1604.

115. Linsley, P. S., J. L. Greene, W. Brady, J. Bajorath, J. A. Ledbetter, and R. Peach. 1994. Human B7-1 (CD80) and B7-2 (CD86) bind with similar avidities but distinct kinetics to CD28 and CTLA-4 receptors. Immunity 1:793-801.

116. Brunet, J. F., F. Denizot, M. F. Luciani, M. Roux-Dosseto, M. Suzan, M. G. Mattei, and P. Golstein. 1987. A new member of the immunoglobulin superfamily--CTLA-4. Nature 328:267-270.

117. Chambers, C. A., M. S. Kuhns, J. G. Egen, and J. P. Allison. 2001. CTLA-4-mediated inhibition in regulation of $\mathrm{T}$ cell responses: mechanisms and manipulation in tumor immunotherapy. Annu Rev Immunol 19:565-594.

118. Waterhouse, P., J. M. Penninger, E. Timms, A. Wakeham, A. Shahinian, K. P. Lee, C. B. Thompson, H. Griesser, and T. W. Mak. 1995. Lymphoproliferative disorders with early lethality in mice deficient in Ctla-4. Science 270:985-988. 
119. Tivol, E. A., F. Borriello, A. N. Schweitzer, W. P. Lynch, J. A. Bluestone, and A. H. Sharpe. 1995. Loss of CTLA-4 leads to massive lymphoproliferation and fatal multiorgan tissue destruction, revealing a critical negative regulatory role of CTLA-4. Immunity 3:541-547.

120. van Kooten, C., and J. Banchereau. 1997. Functions of CD40 on B cells, dendritic cells and other cells. Curr Opin Immunol 9:330-337.

121. Bourgeois, C., B. Rocha, and C. Tanchot. 2002. A role for CD40 expression on CD8+ T cells in the generation of CD8+ T cell memory. Science 297:2060-2063.

122. Tomura, M., W. G. Yu, H. J. Ahn, M. Yamashita, Y. F. Yang, S. Ono, T. Hamaoka, T. Kawano, M. Taniguchi, Y. Koezuka, and H. Fujiwara. 1999. A novel function of Valpha14+CD4+NKT cells: stimulation of IL-12 production by antigen-presenting cells in the innate immune system. J Immunol 163:93-101.

123. van Kooten, C., and J. Banchereau. 2000. CD40-CD40 ligand. J Leukoc Biol 67:2-17.

124. Quezada, S. A., L. Z. Jarvinen, E. F. Lind, and R. J. Noelle. 2004. CD40/CD154 interactions at the interface of tolerance and immunity. Annu Rev Immunol 22:307-328.

125. Ridge, J. P., F. Di Rosa, and P. Matzinger. 1998. A conditioned dendritic cell can be a temporal bridge between a CD4+ T-helper and a T-killer cell. Nature 393:474-478.

126. Bennett, S. R., F. R. Carbone, F. Karamalis, R. A. Flavell, J. F. Miller, and W. R. Heath. 1998. Help for cytotoxic-T-cell responses is mediated by CD40 signalling. Nature 393:478-480.

127. Schoenberger, S. P., R. E. Toes, E. I. van der Voort, R. Offringa, and C. J. Melief. 1998. T-cell help for cytotoxic T lymphocytes is mediated by CD40-CD40L interactions. Nature 393:480-483.

128. Björck, P., J. Banchereau, and L. Flores-Romo. 1997. CD40 ligation counteracts Fasinduced apoptosis of human dendritic cells. Int Immunol 9:365-372.

129. Medema, J. P., D. H. Schuurhuis, D. Rea, J. van Tongeren, J. de Jong, S. A. Bres, S. Laban, R. E. Toes, M. Toebes, T. N. Schumacher, B. A. Bladergroen, F. Ossendorp, J. A. Kummer, C. J. Melief, and R. Offringa. 2001. Expression of the serpin serine protease inhibitor 6 protects dendritic cells from cytotoxic T lymphocyte-induced apoptosis: differential modulation by T helper type 1 and type 2 cells. $J$ Exp Med 194:657-667.

130. Anderson, D. M., E. Maraskovsky, W. L. Billingsley, W. C. Dougall, M. E. Tometsko, E. R. Roux, M. C. Teepe, R. F. DuBose, D. Cosman, and L. Galibert. 1997. A homologue of the TNF receptor and its ligand enhance T-cell growth and dendritic-cell function. Nature 390:175-179.

131. Josien, R., B. R. Wong, H. L. Li, R. M. Steinman, and Y. Choi. 1999. TRANCE, a TNF family member, is differentially expressed on $\mathrm{T}$ cell subsets and induces cytokine production in dendritic cells. J Immunol 162:2562-2568.

132. Wong, B. R., R. Josien, S. Y. Lee, B. Sauter, H. L. Li, R. M. Steinman, and Y. Choi. 1997. TRANCE (tumor necrosis factor [TNF]-related activation-induced cytokine), a new TNF family member predominantly expressed in T cells, is a dendritic cellspecific survival factor. J Exp Med 186:2075-2080.

133. Bertram, E. M., P. Lau, and T. H. Watts. 2002. Temporal segregation of 4-1BB versus CD28-mediated costimulation: 4-1BB ligand influences T cell numbers late in the primary response and regulates the size of the $\mathrm{T}$ cell memory response following influenza infection. J Immunol 168:3777-3785. 
134. Carreno, B. M., and M. Collins. 2002. The B7 family of ligands and its receptors: new pathways for costimulation and inhibition of immune responses. Annu Rev Immunol 20:29-53.

135. Cannons, J. L., P. Lau, B. Ghumman, M. A. DeBenedette, H. Yagita, K. Okumura, and T. H. Watts. 2001. 4-1BB ligand induces cell division, sustains survival, and enhances effector function of CD4 and CD8 T cells with similar efficacy. J Immunol 167:13131324.

136. Chen, A. I., A. J. McAdam, J. E. Buhlmann, S. Scott, M. L. Lupher, E. A. Greenfield, P. R. Baum, W. C. Fanslow, D. M. Calderhead, G. J. Freeman, and A. H. Sharpe. 1999. Ox40-ligand has a critical costimulatory role in dendritic cell:T cell interactions. Immunity 11:689-698.

137. Dawicki, W., and T. H. Watts. 2004. Expression and function of 4-1BB during CD4 versus CD8 T cell responses in vivo. Eur J Immunol 34:743-751.

138. Gramaglia, I., A. D. Weinberg, M. Lemon, and M. Croft. 1998. Ox-40 ligand: a potent costimulatory molecule for sustaining primary CD4 T cell responses. J Immunol 161:6510-6517.

139. Ohshima, Y., Y. Tanaka, H. Tozawa, Y. Takahashi, C. Maliszewski, and G. Delespesse. 1997. Expression and function of OX40 ligand on human dendritic cells. $J$ Immunol 159:3838-3848.

140. Rogers, P. R., J. Song, I. Gramaglia, N. Killeen, and M. Croft. 2001. OX40 promotes $\mathrm{Bcl}-\mathrm{xL}$ and Bcl-2 expression and is essential for long-term survival of CD4 T cells. Immunity 15:445-455.

141. Shuford, W. W., K. Klussman, D. D. Tritchler, D. T. Loo, J. Chalupny, A. W. Siadak, T. J. Brown, J. Emswiler, H. Raecho, C. P. Larsen, T. C. Pearson, J. A. Ledbetter, A. Aruffo, and R. S. Mittler. 1997. 4-1BB costimulatory signals preferentially induce $\mathrm{CD} 8+\mathrm{T}$ cell proliferation and lead to the amplification in vivo of cytotoxic $\mathrm{T}$ cell responses. J Exp Med 186:47-55.

142. Kamath, A. T., J. Pooley, M. A. O'Keeffe, D. Vremec, Y. Zhan, A. M. Lew, A. D'Amico, L. Wu, D. F. Tough, and K. Shortman. 2000. The development, maturation, and turnover rate of mouse spleen dendritic cell populations. J Immunol 165:67626770.

143. Ruedl, C., P. Koebel, M. Bachmann, M. Hess, and K. Karjalainen. 2000. Anatomical origin of dendritic cells determines their life span in peripheral lymph nodes. $J$ Immunol 165:4910-4916.

144. Hou, W. S., and L. Van Parijs. 2004. A Bcl-2-dependent molecular timer regulates the lifespan and immunogenicity of dendritic cells. Nat Immunol 5:583-589.

145. De Smedt, T., B. Pajak, G. G. Klaus, R. J. Noelle, J. Urbain, O. Leo, and M. Moser. 1998. Antigen-specific T lymphocytes regulate lipopolysaccharide-induced apoptosis of dendritic cells in vivo. $J$ Immunol 161:4476-4479.

146. Koppi, T. A., T. Tough-Bement, D. M. Lewinsohn, D. H. Lynch, and M. R. Alderson. 1997. CD40 ligand inhibits Fas/CD95-mediated apoptosis of human blood-derived dendritic cells. Eur J Immunol 27:3161-3165.

147. Miga, A. J., S. R. Masters, B. G. Durell, M. Gonzalez, M. K. Jenkins, C. Maliszewski, H. Kikutani, W. F. Wade, and R. J. Noelle. 2001. Dendritic cell longevity and T cell persistence is controlled by CD154-CD40 interactions. Eur J Immunol 31:959-965.

148. Degli-Esposti, M. A., and M. J. Smyth. 2005. Close encounters of different kinds: dendritic cells and NK cells take centre stage. Nat Rev Immunol 5:112-124. 
149. Hayakawa, Y., V. Screpanti, H. Yagita, A. Grandien, H. G. Ljunggren, M. J. Smyth, and B. J. Chambers. 2004. NK cell TRAIL eliminates immature dendritic cells in vivo and limits dendritic cell vaccination efficacy. J Immunol 172:123-129.

150. McCarthy, C., D. Shepherd, S. Fleire, V. S. Stronge, M. Koch, P. A. Illarionov, G. Bossi, M. Salio, G. Denkberg, F. Reddington, A. Tarlton, B. G. Reddy, R. R. Schmidt, Y. Reiter, G. M. Griffiths, P. A. van der Merwe, G. S. Besra, E. Y. Jones, F. D. Batista, and V. Cerundolo. 2007. The length of lipids bound to human CD1d molecules modulates the affinity of NKT cell TCR and the threshold of NKT cell activation. $J$ Exp Med 204:1131-1144.

151. Nicol, A., M. Nieda, Y. Koezuka, S. Porcelli, K. Suzuki, K. Tadokoro, S. Durrant, and T. Juji. 2000. Dendritic cells are targets for human invariant Valpha24+ natural killer T-cell cytotoxic activity: an important immune regulatory function. Exp Hematol 28:276-282.

152. Nieda, M., A. Kikuchi, A. Nicol, Y. Koezuka, Y. Ando, S. Ishihara, N. Lapteva, T. Yabe, K. Tokunaga, K. Tadokoro, and T. Juji. 2001. Dendritic cells rapidly undergo apoptosis in vitro following culture with activated CD4+ Valpha24 natural killer T cells expressing CD40L. Immunology 102:137-145.

153. Palmowski, M. J., E. M. Choi, I. F. Hermans, S. C. Gilbert, J. L. Chen, U. Gileadi, M. Salio, A. Van Pel, S. Man, E. Bonin, P. Liljestrom, P. R. Dunbar, and V. Cerundolo. 2002. Competition between CTL narrows the immune response induced by primeboost vaccination protocols. J Immunol 168:4391-4398.

154. Wong, P., and E. G. Pamer. 2003. CD8 T cell responses to infectious pathogens. Annu Rev Immunol 21:29-70.

155. Yang, J., S. P. Huck, R. S. McHugh, I. F. Hermans, and F. Ronchese. 2006. Perforindependent elimination of dendritic cells regulates the expansion of antigen-specific CD8+ T cells in vivo. Proc Natl Acad Sci USA 103:147-152.

156. Belz, G. T., L. Zhang, M. D. Lay, F. Kupresanin, and M. P. Davenport. 2007. Killer T cells regulate antigen presentation for early expansion of memory, but not naive, CD8+ T cell. Proc Natl Acad Sci USA 104:6341-6346.

157. Chen, M., L. Huang, Z. Shabier, and J. Wang. 2007. Regulation of the lifespan in dendritic cell subsets. Mol Immunol 44:2558-2565.

158. Chen, M., L. Huang, and J. Wang. 2007. Deficiency of Bim in dendritic cells contributes to overactivation of lymphocytes and autoimmunity. Blood 109:4360-4367.

159. Hon, H., E. B. Rucker, L. Hennighausen, and J. Jacob. 2004. bcl-xL is critical for dendritic cell survival in vivo. J Immunol 173:4425-4432.

160. Kim, T. W., C. F. Hung, D. Boyd, J. Juang, L. He, J. W. Kim, J. M. Hardwick, and T. C. Wu. 2003. Enhancing DNA vaccine potency by combining a strategy to prolong dendritic cell life with intracellular targeting strategies. J Immunol 171:2970-2976.

161. Kim, T. W., C. F. Hung, M. Ling, J. Juang, L. He, J. M. Hardwick, S. Kumar, and T. C. Wu. 2003. Enhancing DNA vaccine potency by coadministration of DNA encoding antiapoptotic proteins. J Clin Invest 112:109-117.

162. Kim, T. W., J. H. Lee, L. He, D. A. Boyd, J. M. Hardwick, C. F. Hung, and T. C. Wu. 2005. Modification of professional antigen-presenting cells with small interfering RNA in vivo to enhance cancer vaccine potency. Cancer Res 65:309-316.

163. Wang, J., L. Zheng, A. Lobito, F. K. Chan, J. Dale, M. Sneller, X. Yao, J. M. Puck, S. E. Straus, and M. J. Lenardo. 1999. Inherited human Caspase 10 mutations underlie 
defective lymphocyte and dendritic cell apoptosis in autoimmune lymphoproliferative syndrome type II. Cell 98:47-58.

164. Chen, M., Y. H. Wang, Y. Wang, L. Huang, H. Sandoval, Y. J. Liu, and J. Wang. 2006. Dendritic cell apoptosis in the maintenance of immune tolerance. Science 311:1160-1164.

165. Inaba, K., M. Inaba, N. Romani, H. Aya, M. Deguchi, S. Ikehara, S. Muramatsu, and R. M. Steinman. 1992. Generation of large numbers of dendritic cells from mouse bone marrow cultures supplemented with granulocyte/macrophage colony-stimulating factor. J Exp Med 176:1693-1702.

166. Markowicz, S., and E. G. Engleman. 1990. Granulocyte-macrophage colonystimulating factor promotes differentiation and survival of human peripheral blood dendritic cells in vitro. $J$ Clin Invest 85:955-961.

167. Banchereau, J., and K. A. Palucka. 2005. Dendritic cells as therapeutic vaccines against cancer. Nat Rev Immunol 5:296-306.

168. Palucka, A. K., H. Ueno, J. Fay, and J. Banchereau. 2008. Dendritic cells: a critical player in cancer therapy? J Immunother 31:793-805.

169. Hermans, I. F., D. S. Ritchie, J. Yang, J. M. Roberts, and F. Ronchese. 2000. CD8+ T cell-dependent elimination of dendritic cells in vivo limits the induction of antitumor immunity. J Immunol 164:3095-3101.

170. Gilboa, E. 2007. DC-based cancer vaccines. J Clin Invest 117:1195-1203.

171. von Boehmer, H. 1988. The developmental biology of T lymphocytes. Annu Rev Immunol 6:309-326.

172. Surh, C. D., and J. Sprent. 1994. T-cell apoptosis detected in situ during positive and negative selection in the thymus. Nature 372:100-103.

173. Ahmed, R., and D. Gray. 1996. Immunological memory and protective immunity: understanding their relation. Science 272:54-60.

174. Williams, M. A., and M. J. Bevan. 2007. Effector and memory CTL differentiation. Annu Rev Immunol 25:171-192.

175. Bousso, P., J. P. Levraud, P. Kourilsky, and J. P. Abastado. 1999. The composition of a primary $\mathrm{T}$ cell response is largely determined by the timing of recruitment of individual T cell clones. J Exp Med 189:1591-1600.

176. Hou, S., L. Hyland, K. W. Ryan, A. Portner, and P. C. Doherty. 1994. Virus-specific CD8+ T-cell memory determined by clonal burst size. Nature 369:652-654.

177. Murali-Krishna, K., J. D. Altman, M. Suresh, D. J. Sourdive, A. J. Zajac, J. D. Miller, J. Slansky, and R. Ahmed. 1998. Counting antigen-specific CD8 T cells: a reevaluation of bystander activation during viral infection. Immunity 8:177-187.

178. Whitmire, J. K., M. S. Asano, K. Murali-Krishna, M. Suresh, and R. Ahmed. 1998. Long-term CD4 Th1 and Th2 memory following acute lymphocytic choriomeningitis virus infection. $J$ Virol 72:8281-8288.

179. Mescher, M. F., J. M. Curtsinger, P. Agarwal, K. A. Casey, M. Gerner, C. D. Hammerbeck, F. Popescu, and Z. Xiao. 2006. Signals required for programming effector and memory development by CD8+ T cells. Immunol Rev 211:81-92.

180. Curtsinger, J. M., C. S. Schmidt, A. Mondino, D. C. Lins, R. M. Kedl, M. K. Jenkins, and M. F. Mescher. 1999. Inflammatory cytokines provide a third signal for activation of naive CD4+ and CD8+ T cells. J Immunol 162:3256-3262. 
181. Schmidt, C. S., and M. F. Mescher. 1999. Adjuvant effect of IL-12: conversion of peptide antigen administration from tolerizing to immunizing for $\mathrm{CD} 8+\mathrm{T}$ cells in vivo. J Immunol 163:2561-2567.

182. Schmidt, C. S., and M. F. Mescher. 2002. Peptide antigen priming of naive, but not memory, CD8 $\mathrm{T}$ cells requires a third signal that can be provided by IL-12. J Immunol 168:5521-5529.

183. Valenzuela, J. O., C. D. Hammerbeck, and M. F. Mescher. 2005. Cutting edge: Bcl-3 up-regulation by signal 3 cytokine (IL-12) prolongs survival of antigen-activated CD8 T cells. J Immunol 174:600-604.

184. Curtsinger, J. M., J. O. Valenzuela, P. Agarwal, D. Lins, and M. F. Mescher. 2005. Type I IFNs provide a third signal to CD8 T cells to stimulate clonal expansion and differentiation. J Immunol 174:4465-4469.

185. Kolumam, G. A., S. Thomas, L. J. Thompson, J. Sprent, and K. Murali-Krishna. 2005. Type I interferons act directly on CD8 T cells to allow clonal expansion and memory formation in response to viral infection. J Exp Med 202:637-650.

186. Le Bon, A., V. Durand, E. Kamphuis, C. Thompson, S. Bulfone-Paus, C. Rossmann, U. Kalinke, and D. F. Tough. 2006. Direct stimulation of T cells by type I IFN enhances the CD8+ T cell response during cross-priming. J Immunol 176:4682-4689.

187. Fujii, S., K. Liu, C. Smith, A. J. Bonito, and R. M. Steinman. 2004. The linkage of innate to adaptive immunity via maturing dendritic cells in vivo requires CD40 ligation in addition to antigen presentation and CD80/86 costimulation. $J$ Exp Med 199:16071618.

188. Kaech, S. M., and R. Ahmed. 2001. Memory CD8+ T cell differentiation: initial antigen encounter triggers a developmental program in naïve cells. Nat Immunol 2:415-422.

189. Mercado, R., S. Vijh, S. E. Allen, K. Kerksiek, I. M. Pilip, and E. G. Pamer. 2000. Early programming of $\mathrm{T}$ cell populations responding to bacterial infection. J Immunol 165:6833-6839.

190. van Stipdonk, M. J., E. E. Lemmens, and S. P. Schoenberger. 2001. Naïve CTLs require a single brief period of antigenic stimulation for clonal expansion and differentiation. Nat Immunol 2:423-429.

191. Wong, P., and E. G. Pamer. 2001. Cutting edge: antigen-independent CD8 T cell proliferation. J Immunol 166:5864-5868.

192. Germain, R., M. J. Miller, M. L. Dustin, and M. C. Nussenzweig. 2006. Dynamic imaging of the immune system: progress, pitfalls and promise. Nat Rev Immunol 6:497-507.

193. Mempel, T., S. Henrickson, and U. Von Andrian. 2004. T-cell priming by dendritic cells in lymph nodes occurs in three distinct phases. Nature 427:154-159.

194. Bousso, P. 2008. T-cell activation by dendritic cells in the lymph node: lessons from the movies. Nat Rev Immunol 8:675-684.

195. Badovinac, V. P., B. Porter, and J. T. Harty. 2002. Programmed contraction of CD8(+) T cells after infection. Nat Immunol 3:619-626.

196. Harty, J. T., A. R. Tvinnereim, and D. W. White. 2000. CD8+ T cell effector mechanisms in resistance to infection. Annu Rev Immunol 18:275-308.

197. Sallusto, F., D. Lenig, R. Förster, M. Lipp, and A. Lanzavecchia. 1999. Two subsets of memory T lymphocytes with distinct homing potentials and effector functions. Nature 401:708-712. 
198. Masopust, D., V. Vezys, A. L. Marzo, and L. Lefrançois. 2001. Preferential localization of effector memory cells in nonlymphoid tissue. Science 291:2413-2417.

199. Unsoeld, H., and H. Pircher. 2005. Complex memory T-cell phenotypes revealed by coexpression of CD62L and CCR7. J Virol 79:4510-4513.

200. Wherry, E. J., V. Teichgräber, T. C. Becker, D. Masopust, S. M. Kaech, R. Antia, U. Von Andrian, and R. Ahmed. 2003. Lineage relationship and protective immunity of memory CD8 T cell subsets. Nat Immunol 4:225-234.

201. Kaech, S. M., and E. J. Wherry. 2007. Heterogeneity and cell-fate decisions in effector and memory CD8+ T cell differentiation during viral infection. Immunity 27:393-405.

202. Kaech, S. M., E. J. Wherry, and R. Ahmed. 2002. Effector and memory T-cell differentiation: implications for vaccine development. Nat Rev Immunol 2:251-262.

203. Jacob, J., and D. Baltimore. 1999. Modelling T-cell memory by genetic marking of memory T cells in vivo. Nature 399:593-597.

204. Kaech, S. M., S. Hemby, E. Kersh, and R. Ahmed. 2002. Molecular and functional profiling of memory CD8 T cell differentiation. Cell 111:837-851.

205. Opferman, J. T., B. T. Ober, and P. G. Ashton-Rickardt. 1999. Linear differentiation of cytotoxic effectors into memory T lymphocytes. Science 283:1745-1748.

206. Chang, J. T., V. R. Palanivel, I. Kinjyo, F. Schambach, A. M. Intlekofer, A. Banerjee, S. A. Longworth, K. E. Vinup, P. Mrass, J. Oliaro, N. Killeen, J. S. Orange, S. M. Russell, W. Weninger, and S. L. Reiner. 2007. Asymmetric T lymphocyte division in the initiation of adaptive immune responses. Science 315:1687-1691.

207. Lanzavecchia, A., and F. Sallusto. 2000. Dynamics of T lymphocyte responses: intermediates, effectors, and memory cells. Science 290:92-97.

208. Manjunath, N., P. Shankar, J. Wan, W. Weninger, M. A. Crowley, K. Hieshima, T. A. Springer, X. Fan, H. Shen, J. Lieberman, and U. H. von Andrian. 2001. Effector differentiation is not prerequisite for generation of memory cytotoxic T lymphocytes. $J$ Clin Invest 108:871-878.

209. Wong, P., M. Lara-Tejero, A. Ploss, I. Leiner, and E. G. Pamer. 2004. Rapid development of T cell memory. J Immunol 172:7239-7245.

210. Zhang, M., S. Byrne, N. Liu, Y. Wang, A. Oxenius, and P. G. Ashton-Rickardt. 2007. Differential survival of cytotoxic $\mathrm{T}$ cells and memory cell precursors. J Immunol 178:3483-3491.

211. Busch, D. H., I. M. Pilip, S. Vijh, and E. G. Pamer. 1998. Coordinate regulation of complex T cell populations responding to bacterial infection. Immunity 8:353-362.

212. Bachmann, M. F., M. Barner, A. Viola, and M. Kopf. 1999. Distinct kinetics of cytokine production and cytolysis in effector and memory T cells after viral infection. Eur J Immunol 29:291-299.

213. Veiga-Fernandes, H., U. Walter, C. Bourgeois, A. McLean, and B. Rocha. 2000. Response of naïve and memory CD8+ T cells to antigen stimulation in vivo. Nat Immunol 1:47-53.

214. Teague, T. K., D. Hildeman, R. M. Kedl, T. Mitchell, W. Rees, B. C. Schaefer, J. Bender, J. Kappler, and P. Marrack. 1999. Activation changes the spectrum but not the diversity of genes expressed by T cells. Proc Natl Acad Sci USA 96:12691-12696.

215. Yang, Y., J. F. Chang, J. R. Parnes, and C. G. Fathman. 1998. T cell receptor (TCR) engagement leads to activation-induced splicing of tumor necrosis factor (TNF) nuclear pre-mRNA. J Exp Med 188:247-254. 
216. Badovinac, V. P., and J. T. Harty. 2000. Adaptive immunity and enhanced CD8+ T cell response to Listeria monocytogenes in the absence of perforin and IFN-gamma. $J$ Immunol 164:6444-6452.

217. Grayson, J. M., K. Murali-Krishna, J. D. Altman, and R. Ahmed. 2001. Gene expression in antigen-specific CD8+ T cells during viral infection. J Immunol 166:795799.

218. Moser, B., and P. Loetscher. 2001. Lymphocyte traffic control by chemokines. Nat Immunol 2:123-128.

219. Berke, G. 1995. The CTL's kiss of death. Cell 81:9-12.

220. Wajant, H., K. Pfizenmaier, and P. Scheurich. 2003. Tumor necrosis factor signaling. Cell Death Differ 10:45-65.

221. Samuel, C. E. 2001. Antiviral actions of interferons. Clin Microbiol Rev 14:778-809, table of contents.

222. Slifka, M. K., and J. L. Whitton. 2000. Antigen-specific regulation of T cell-mediated cytokine production. Immunity 12:451-457.

223. Lavrik, I., A. Golks, and P. H. Krammer. 2005. Death receptor signaling. J Cell Sci 118:265-267.

224. Waring, P., and A. Müllbacher. 1999. Cell death induced by the Fas/Fas ligand pathway and its role in pathology. Immunol Cell Biol 77:312-317.

225. Nagata, S., and P. Golstein. 1995. The Fas death factor. Science 267:1449-1456.

226. Siegel, R. M., F. K. M. Chan, H. J. Chun, and M. J. Lenardo. 2000. The multifaceted role of Fas signaling in immune cell homeostasis and autoimmunity. Nat Immunol $1: 469-474$.

227. Liu, C. C., B. Perussia, Z. A. Cohn, and J. D. Young. 1986. Identification and characterization of a pore-forming protein of human peripheral blood natural killer cells. J Exp Med 164:2061-2076.

228. Masson, D., and J. Tschopp. 1985. Isolation of a lytic, pore-forming protein (perforin) from cytolytic T-lymphocytes. J Biol Chem 260:9069-9072.

229. Podack, E. R., J. D. Young, and Z. A. Cohn. 1985. Isolation and biochemical and functional characterization of perforin 1 from cytolytic T-cell granules. Proc Natl Acad Sci USA 82:8629-8633.

230. Young, J. D., Z. A. Cohn, and E. R. Podack. 1986. The ninth component of complement and the pore-forming protein (perforin 1) from cytotoxic T cells: structural, immunological, and functional similarities. Science 233:184-190.

231. Tschopp, J., D. Masson, and K. K. Stanley. 1986. Structural/functional similarity between proteins involved in complement- and cytotoxic T-lymphocyte-mediated cytolysis. Nature 322:831-834.

232. Bolitho, P., I. Voskoboinik, J. A. Trapani, and M. J. Smyth. 2007. Apoptosis induced by the lymphocyte effector molecule perforin. Curr Opin Immunol 19:339-347.

233. Lichtenheld, M. G., K. J. Olsen, P. Lu, D. M. Lowrey, A. Hameed, H. Hengartner, and E. R. Podack. 1988. Structure and function of human perforin. Nature 335:448-451.

234. Baran, K., M. Dunstone, J. Chia, A. Ciccone, K. A. Browne, C. J. Clarke, N. Lukoyanova, H. Saibil, J. C. Whisstock, I. Voskoboinik, and J. A. Trapani. 2009. The molecular basis for perforin oligomerization and transmembrane pore assembly. Immunity 30:684-695. 
235. Gumperz, J., S. Miyake, T. Yamamura, and M. B. Brenner. 2002. Functionally distinct subsets of CD1d-restricted natural killer T cells revealed by CD1d tetramer staining. $J$ Exp Med 195:625-636.

236. Kägi, D., B. Ledermann, K. Bürki, R. M. Zinkernagel, and H. Hengartner. 1996. Molecular mechanisms of lymphocyte-mediated cytotoxicity and their role in immunological protection and pathogenesis in vivo. Annu Rev Immunol 14:207-232.

237. Nakata, M., A. Kawasaki, M. Azuma, K. Tsuji, H. Matsuda, Y. Shinkai, H. Yagita, and K. Okumura. 1992. Expression of perforin and cytolytic potential of human peripheral blood lymphocyte subpopulations. Int Immunol 4:1049-1054.

238. Peters, P. J., J. Borst, V. Oorschot, M. Fukuda, O. Krähenbühl, J. Tschopp, J. W. Slot, and H. J. Geuze. 1991. Cytotoxic T lymphocyte granules are secretory lysosomes, containing both perforin and granzymes. J Exp Med 173:1099-1109.

239. Lieberman, J. 2003. The ABCs of granule-mediated cytotoxicity: new weapons in the arsenal. Nat Rev Immunol 3:361-370.

240. Lu, P., J. A. Garcia-Sanz, M. G. Lichtenheld, and E. R. Podack. 1992. Perforin expression in human peripheral blood mononuclear cells. Definition of an IL-2independent pathway of perforin induction in CD8+ T cells. J Immunol 148:33543360 .

241. Zhang, J., I. Scordi, M. J. Smyth, and M. G. Lichtenheld. 1999. Interleukin 2 receptor signaling regulates the perforin gene through signal transducer and activator of transcription (Stat)5 activation of two enhancers. J Exp Med 190:1297-1308.

242. Salcedo, T. W., L. Azzoni, S. F. Wolf, and B. Perussia. 1993. Modulation of perforin and granzyme messenger RNA expression in human natural killer cells. J Immunol 151:2511-2520.

243. Stinchcombe, J. C., and G. M. Griffiths. 2007. Secretory mechanisms in cell-mediated cytotoxicity. Annu Rev Cell Dev Biol 23:495-517.

244. Kägi, D., B. Ledermann, K. Bürki, P. Seiler, B. Odermatt, K. J. Olsen, E. R. Podack, R. M. Zinkernagel, and H. Hengartner. 1994. Cytotoxicity mediated by T cells and natural killer cells is greatly impaired in perforin-deficient mice. Nature 369:31-37.

245. Kojima, H., N. Shinohara, S. Hanaoka, Y. Someya-Shirota, Y. Takagaki, H. Ohno, T. Saito, T. Katayama, H. Yagita, and K. Okumura. 1994. Two distinct pathways of specific killing revealed by perforin mutant cytotoxic T lymphocytes. Immunity 1:357364.

246. Liu, C. C., C. M. Walsh, and J. D. Young. 1995. Perforin: structure and function. Immunol Today 16:194-201.

247. Lowin, B., F. Beermann, A. Schmidt, and J. Tschopp. 1994. A null mutation in the perforin gene impairs cytolytic T lymphocyte- and natural killer cell-mediated cytotoxicity. Proc Natl Acad Sci USA 91:11571-11575.

248. Walsh, C. M., M. Matloubian, C. C. Liu, R. Ueda, C. G. Kurahara, J. L. Christensen, M. T. Huang, J. D. Young, R. Ahmed, and W. R. Clark. 1994. Immune function in mice lacking the perforin gene. Proc Natl Acad Sci USA 91:10854-10858.

249. Nakajima, H., H. L. Park, and P. A. Henkart. 1995. Synergistic roles of granzymes A and $\mathrm{B}$ in mediating target cell death by rat basophilic leukemia mast cell tumors also expressing cytolysin/perforin. J Exp Med 181:1037-1046.

250. Shiver, J. W., L. Su, and P. A. Henkart. 1992. Cytotoxicity with target DNA breakdown by rat basophilic leukemia cells expressing both cytolysin and granzyme A. Cell 71:315-322. 
251. Froelich, C. J., K. Orth, J. Turbov, P. Seth, R. Gottlieb, B. Babior, G. M. Shah, R. C. Bleackley, V. M. Dixit, and W. Hanna. 1996. New paradigm for lymphocyte granulemediated cytotoxicity. Target cells bind and internalize granzyme B, but an endosomolytic agent is necessary for cytosolic delivery and subsequent apoptosis. $J$ Biol Chem 271:29073-29079.

252. Metkar, S. S., B. Wang, M. Aguilar-Santelises, S. M. Raja, L. Uhlin-Hansen, E. Podack, J. A. Trapani, and C. J. Froelich. 2002. Cytotoxic cell granule-mediated apoptosis: perforin delivers granzyme B-serglycin complexes into target cells without plasma membrane pore formation. Immunity 16:417-428.

253. Keefe, D., L. Shi, S. Feske, R. Massol, F. Navarro, T. Kirchhausen, and J. Lieberman. 2005. Perforin triggers a plasma membrane-repair response that facilitates CTL induction of apoptosis. Immunity 23:249-262.

254. Pipkin, M. E., and J. Lieberman. 2007. Delivering the kiss of death: progress on understanding how perforin works. Curr Opin Immunol 19:301-308.

255. Motyka, B., G. Korbutt, M. J. Pinkoski, J. A. Heibein, A. Caputo, M. Hobman, M. Barry, I. Shostak, T. Sawchuk, C. F. Holmes, J. Gauldie, and R. C. Bleackley. 2000. Mannose 6-phosphate/insulin-like growth factor II receptor is a death receptor for granzyme B during cytotoxic T cell-induced apoptosis. Cell 103:491-500.

256. Dressel, R., S. M. Raja, S. Höning, T. Seidler, C. J. Froelich, K. von Figura, and E. Günther. 2004. Granzyme-mediated cytotoxicity does not involve the mannose 6phosphate receptors on target cells. J Biol Chem 279:20200-20210.

257. Shi, L., D. Keefe, E. Durand, H. Feng, D. Zhang, and J. Lieberman. 2005. Granzyme B binds to target cells mostly by charge and must be added at the same time as perforin to trigger apoptosis. J Immunol 174:5456-5461.

258. Trapani, J. A., V. R. Sutton, K. Y. Thia, Y. Q. Li, C. J. Froelich, D. A. Jans, M. S. Sandrin, and K. A. Browne. 2003. A clathrin/dynamin- and mannose-6-phosphate receptor-independent pathway for granzyme B-induced cell death. J Cell Biol 160:223233.

259. Shi, L., S. Mai, S. Israels, K. Browne, J. A. Trapani, and A. H. Greenberg. 1997. Granzyme B (GraB) autonomously crosses the cell membrane and perforin initiates apoptosis and GraB nuclear localization. J Exp Med 185:855-866.

260. Trapani, J. A., and M. J. Smyth. 2002. Functional significance of the perforin/granzyme cell death pathway. Nat Rev Immunol 2:735-747.

261. Makino, Y., R. Kanno, T. Ito, K. Higashino, and M. Taniguchi. 1995. Predominant expression of invariant $\mathrm{V}$ alpha $14+\mathrm{TCR}$ alpha chain in NK1.1+ T cell populations. Int Immunol 7:1157-1161.

262. Godfrey, D. I., H. R. MacDonald, M. Kronenberg, M. J. Smyth, and L. Van Kaer. 2004. NKT cells: what's in a name? Nat Rev Immunol 4:231-237.

263. Kronenberg, M. 2005. Toward an understanding of NKT cell biology: progress and paradoxes. Annu Rev Immunol 23:877-900.

264. Benlagha, K., A. Weiss, A. Beavis, L. Teyton, and A. Bendelac. 2000. In vivo identification of glycolipid antigen-specific T cells using fluorescent CD1d tetramers. $J$ Exp Med 191:1895-1903.

265. Karadimitris, A., S. Gadola, M. Altamirano, D. Brown, A. Woolfson, P. Klenerman, J. L. Chen, Y. Koezuka, I. A. Roberts, D. A. Price, G. Dusheiko, C. Milstein, A. Fersht, L. Luzzatto, and V. Cerundolo. 2001. Human CD1d-glycolipid tetramers generated by in vitro oxidative refolding chromatography. Proc Natl Acad Sci USA 98:3294-3298. 
266. Matsuda, J. L., O. V. Naidenko, L. Gapin, T. Nakayama, M. Taniguchi, C. R. Wang, Y. Koezuka, and M. Kronenberg. 2000. Tracking the response of natural killer T cells to a glycolipid antigen using CD1d tetramers. J Exp Med 192:741-754.

267. Cardell, S., S. Tangri, S. Chan, M. Kronenberg, C. Benoist, and D. Mathis. 1995. CD1restricted CD4+ T cells in major histocompatibility complex class II-deficient mice. $J$ Exp Med 182:993-1004.

268. Chen, H., and W. E. Paul. 1997. Cultured NK1.1+ CD4+ T cells produce large amounts of IL-4 and IFN-gamma upon activation by anti-CD3 or CD1. J Immunol 159:2240-2249.

269. Yoshimoto, T., and W. E. Paul. 1994. CD4pos, NK1.1pos T cells promptly produce interleukin 4 in response to in vivo challenge with anti-CD3. J Exp Med 179:12851295.

270. Matsuda, J. L., L. Gapin, J. L. Baron, S. Sidobre, D. B. Stetson, M. Mohrs, R. M. Locksley, and M. Kronenberg. 2003. Mouse V alpha 14i natural killer T cells are resistant to cytokine polarization in vivo. Proc Natl Acad Sci USA 100:8395-8400.

271. Stetson, D. B., M. Mohrs, R. L. Reinhardt, J. L. Baron, Z. E. Wang, L. Gapin, M. Kronenberg, and R. M. Locksley. 2003. Constitutive cytokine mRNAs mark natural killer (NK) and NK T cells poised for rapid effector function. J Exp Med 198:10691076.

272. Matsuda, J. L., T. Mallevaey, J. Scott-Browne, and L. Gapin. 2008. CD1d-restricted iNKT cells, the 'Swiss-Army knife' of the immune system. Curr Opin Immunol 20:358-368.

273. Godfrey, D. I., K. J. Hammond, L. D. Poulton, M. J. Smyth, and A. G. Baxter. 2000. NKT cells: facts, functions and fallacies. Immunol Today 21:573-583.

274. Bendelac, A., P. B. Savage, and L. Teyton. 2007. The biology of NKT cells. Annu Rev Immunol 25:297-336.

275. Godfrey, D. I., and M. Kronenberg. 2004. Going both ways: immune regulation via CD1d-dependent NKT cells. J Clin Invest 114:1379-1388.

276. Kronenberg, M., and L. Gapin. 2002. The unconventional lifestyle of NKT cells. Nat Rev Immunol 2:557-568.

277. Coles, M. C., and D. H. Raulet. 2000. NK1.1+ T cells in the liver arise in the thymus and are selected by interactions with class I molecules on CD4+CD8+ cells. J Immunol 164:2412-2418.

278. Tilloy, F., J. P. Di Santo, A. Bendelac, and O. Lantz. 1999. Thymic dependence of invariant V alpha 14+ natural killer-T cell development. Eur J Immunol 29:3313-3318.

279. Hammond, K. J., S. B. Pelikan, N. Y. Crowe, E. Randle-Barrett, T. Nakayama, M. Taniguchi, M. J. Smyth, I. R. van Driel, R. Scollay, A. G. Baxter, and D. I. Godfrey. 1999. NKT cells are phenotypically and functionally diverse. Eur J Immunol 29:37683781.

280. Pellicci, D. G., K. J. Hammond, A. P. Uldrich, A. G. Baxter, M. J. Smyth, and D. I. Godfrey. 2002. A natural killer T (NKT) cell developmental pathway iInvolving a thymus-dependent NK1.1(-)CD4(+) CD1d-dependent precursor stage. J Exp Med 195:835-844.

281. Bezbradica, J. S., T. Hill, A. K. Stanic, L. Van Kaer, and S. Joyce. 2005. Commitment toward the natural $\mathrm{T}$ (iNKT) cell lineage occurs at the CD4+8+ stage of thymic ontogeny. Proc Natl Acad Sci USA 102:5114-5119. 
282. Egawa, T., G. Eberl, I. Taniuchi, K. Benlagha, F. Geissmann, L. Hennighausen, A. Bendelac, and D. R. Littman. 2005. Genetic evidence supporting selection of the Valpha14i NKT cell lineage from double-positive thymocyte precursors. Immunity 22:705-716.

283. Gapin, L., J. L. Matsuda, C. D. Surh, and M. Kronenberg. 2001. NKT cells derive from double-positive thymocytes that are positively selected by CD1d. Nat Immunol 2:971978.

284. Bendelac, A. 1995. Positive selection of mouse NK1+ T cells by CD1-expressing cortical thymocytes. J Exp Med 182:2091-2096.

285. Wei, D. G., H. Lee, S. H. Park, L. Beaudoin, L. Teyton, A. Lehuen, and A. Bendelac. 2005. Expansion and long-range differentiation of the NKT cell lineage in mice expressing CD1d exclusively on cortical thymocytes. J Exp Med 202:239-248.

286. Godfrey, D. I., and S. P. Berzins. 2007. Control points in NKT-cell development. Nat Rev Immunol 7:505-518.

287. Benlagha, K., D. G. Wei, J. Veiga, L. Teyton, and A. Bendelac. 2005. Characterization of the early stages of thymic NKT cell development. J Exp Med 202:485-492.

288. Benlagha, K., T. Kyin, A. Beavis, L. Teyton, and A. Bendelac. 2002. A thymic precursor to the NK T cell lineage. Science 296:553-555.

289. McNab, F. W., S. P. Berzins, D. G. Pellicci, K. Kyparissoudis, K. Field, M. J. Smyth, and D. I. Godfrey. 2005. The influence of CD1d in postselection NKT cell maturation and homeostasis. J Immunol 175:3762-3768.

290. Davodeau, F., M. A. Peyrat, A. Necker, R. Dominici, F. Blanchard, C. Leget, J. Gaschet, P. Costa, Y. Jacques, A. Godard, H. Vie, A. Poggi, F. Romagné, and M. Bonneville. 1997. Close phenotypic and functional similarities between human and murine alphabeta T cells expressing invariant TCR alpha-chains. J Immunol 158:56035611.

291. Masuda, K., Y. Makino, J. Cui, T. Ito, T. Tokuhisa, Y. Takahama, H. Koseki, K. Tsuchida, T. Koike, H. Moriya, M. Amano, and M. Taniguchi. 1997. Phenotypes and invariant alpha beta TCR expression of peripheral V alpha 14+ NK T cells. J Immunol 158:2076-2082.

292. Crowe, N. Y., J. M. Coquet, S. P. Berzins, K. Kyparissoudis, R. Keating, D. G. Pellicci, Y. Hayakawa, D. I. Godfrey, and M. J. Smyth. 2005. Differential antitumor immunity mediated by NKT cell subsets in vivo. J Exp Med 202:1279-1288.

293. Lee, P. T., K. Benlagha, L. Teyton, and A. Bendelac. 2002. Distinct functional lineages of human V(alpha)24 natural killer T cells. J Exp Med 195:637-641.

294. Coquet, J. M., S. Chakravarti, K. Kyparissoudis, F. W. McNab, L. A. Pitt, B. S. McKenzie, S. P. Berzins, M. J. Smyth, and D. I. Godfrey. 2008. Diverse cytokine production by NKT cell subsets and identification of an IL-17-producing CD4-NK1.1NKT cell population. Proc Natl Acad Sci USA 105:11287-11292.

295. Rogers, P. R., A. Matsumoto, O. Naidenko, M. Kronenberg, T. Mikayama, and S. Kato. 2004. Expansion of human Valpha24+ NKT cells by repeated stimulation with KRN7000. J Immunol Methods 285:197-214.

296. Liu, T. Y., Y. Uemura, M. Suzuki, Y. Narita, S. Hirata, H. Ohyama, O. Ishihara, and S. Matsushita. 2008. Distinct subsets of human invariant NKT cells differentially regulate T helper responses via dendritic cells. Eur J Immunol 38:1012-1023. 
297. Kobayashi, E., K. Motoki, T. Uchida, H. Fukushima, and Y. Koezuka. 1995. KRN7000, a novel immunomodulator, and its antitumor activities. Oncol Res 7:529534.

298. Morita, M., K. Motoki, K. Akimoto, T. Natori, T. Sakai, E. Sawa, K. Yamaji, Y. Koezuka, E. Kobayashi, and H. Fukushima. 1995. Structure-activity relationship of alpha-galactosylceramides against B16-bearing mice. J Med Chem 38:2176-2187.

299. Kawano, T., J. Cui, Y. Koezuka, I. Toura, Y. Kaneko, K. Motoki, H. Ueno, R. Nakagawa, H. Sato, E. Kondo, H. Koseki, and M. Taniguchi. 1997. CD1d-restricted and TCR-mediated activation of valpha14 NKT cells by glycosylceramides. Science 278:1626-1629.

300. Brossay, L., M. Chioda, N. Burdin, Y. Koezuka, G. Casorati, P. Dellabona, and M. Kronenberg. 1998. CD1d-mediated recognition of an alpha-galactosylceramide by natural killer T cells is highly conserved through mammalian evolution. $J$ Exp Med 188:1521-1528.

301. Spada, F. M., Y. Koezuka, and S. A. Porcelli. 1998. CD1d-restricted recognition of synthetic glycolipid antigens by human natural killer T cells. J Exp Med 188:15291534.

302. Kinjo, Y., D. Wu, G. Kim, G. W. Xing, M. A. Poles, D. D. Ho, M. Tsuji, K. Kawahara, C. H. Wong, and M. Kronenberg. 2005. Recognition of bacterial glycosphingolipids by natural killer T cells. Nature 434:520-525.

303. Mattner, J., K. Debord, N. Ismail, R. Goff, C. Cantu, D. Zhou, P. Saint-Mezard, V. Wang, Y. Gao, N. Yin, K. Hoebe, O. Schneewind, D. Walker, B. Beutler, L. Teyton, P. B. Savage, and A. Bendelac. 2005. Exogenous and endogenous glycolipid antigens activate NKT cells during microbial infections. Nature 434:525-529.

304. Sriram, V., W. Du, J. Gervay-Hague, and R. R. Brutkiewicz. 2005. Cell wall glycosphingolipids of Sphingomonas paucimobilis are CD1d-specific ligands for NKT cells. Eur J Immunol 35:1692-1701.

305. Miyamoto, K., S. Miyake, and T. Yamamura. 2001. A synthetic glycolipid prevents autoimmune encephalomyelitis by inducing TH2 bias of natural killer T cells. Nature 413:531-534.

306. Oki, S., A. Chiba, T. Yamamura, and S. Miyake. 2004. The clinical implication and molecular mechanism of preferential IL-4 production by modified glycolipidstimulated NKT cells. J Clin Invest 113:1631-1640.

307. Yu, K. O., J. S. Im, A. Molano, Y. Dutronc, P. A. Illarionov, C. Forestier, N. Fujiwara, I. Arias, S. Miyake, T. Yamamura, Y. T. Chang, G. S. Besra, and S. A. Porcelli. 2005. Modulation of CD1d-restricted NKT cell responses by using N-acyl variants of alphagalactosylceramides. Proc Natl Acad Sci USA 102:3383-3388.

308. Zhou, D., J. Mattner, C. Cantu, N. Schrantz, N. Yin, Y. Gao, Y. Sagiv, K. Hudspeth, Y. P. Wu, T. Yamashita, S. Teneberg, D. Wang, R. L. Proia, S. B. Levery, P. B. Savage, L. Teyton, and A. Bendelac. 2004. Lysosomal glycosphingolipid recognition by NKT cells. Science 306:1786-1789.

309. Van Kaer, L. 2007. NKT cells: T lymphocytes with innate effector functions. Curr Opin Immunol 19:354-364.

310. Yang, O. O., F. K. Racke, P. T. Nguyen, R. Gausling, M. E. Severino, H. F. Horton, M. C. Byrne, J. L. Strominger, and S. B. Wilson. 2000. CD1d on myeloid dendritic cells stimulates cytokine secretion from and cytolytic activity of V alpha $24 \mathrm{~J}$ alpha Q T cells: a feedback mechanism for immune regulation. J Immunol 165:3756-3762. 
311. Matsumoto, G., Y. Omi, U. Lee, T. Nishimura, J. Shindo, and J. M. Penninger. 2000. Adhesion mediated by LFA-1 is required for efficient IL-12-induced NK and NKT cell cytotoxicity. Eur J Immunol 30:3723-3731.

312. Metelitsa, L. S., K. Weinberg, P. Emanuel, and R. C. Seeger. 2003. Expression of CD1d by myelomonocytic leukemias provides a target for cytotoxic NKT cells. Leukemia 17:1068-1077.

313. Nicol, A., M. Nieda, Y. Koezuka, S. Porcelli, K. Suzuki, K. Tadokoro, S. Durrant, and T. Juji. 2000. Human invariant valpha24+ natural killer T cells activated by alphagalactosylceramide (KRN7000) have cytotoxic anti-tumour activity through mechanisms distinct from T cells and natural killer cells. Immunology 99:229-234.

314. Kawano, T., J. Cui, Y. Koezuka, I. Toura, Y. Kaneko, H. Sato, E. Kondo, M. Harada, H. Koseki, T. Nakayama, Y. Tanaka, and M. Taniguchi. 1998. Natural killer-like nonspecific tumor cell lysis mediated by specific ligand-activated Valpha14 NKT cells. Proc Natl Acad Sci USA 95:5690-5693.

315. Mattarollo, S., T. Kenna, M. Nieda, and A. Nicol. 2006. Chemotherapy pretreatment sensitizes solid tumor-derived cell lines to V alpha 24+ NKT cell-mediated cytotoxicity. Int J Cancer 119:1630-1637.

316. Crowe, N. Y., M. J. Smyth, and D. I. Godfrey. 2002. A critical role for natural killer T cells in immunosurveillance of methylcholanthrene-induced sarcomas. $J$ Exp Med 196:119-127.

317. Arase, H., N. Arase, Y. Kobayashi, Y. Nishimune, S. Yonehara, and K. Onoé. 1994. Cytotoxicity of fresh NK1.1+ T cell receptor alpha/beta+ thymocytes against a CD4+8+ thymocyte population associated with intact Fas antigen expression on the target. J Exp Med 180:423-432.

318. Nieda, M., A. Nicol, Y. Koezuka, A. Kikuchi, N. Lapteva, Y. Tanaka, K. Tokunaga, K. Suzuki, N. Kayagaki, H. Yagita, H. Hirai, and T. Juji. 2001. TRAIL expression by activated human CD4(+) V alpha 24NKT cells induces in vitro and in vivo apoptosis of human acute myeloid leukemia cells. Blood 97:2067-2074.

319. Fujii, S., K. Shimizu, C. Smith, L. Bonifaz, and R. M. Steinman. 2003. Activation of natural killer $\mathrm{T}$ cells by alpha-galactosylceramide rapidly induces the full maturation of dendritic cells in vivo and thereby acts as an adjuvant for combined CD4 and CD8 T cell immunity to a coadministered protein. J Exp Med 198:267-279.

320. Hermans, I. F., J. D. Silk, U. Gileadi, M. Salio, B. Mathew, G. Ritter, R. R. Schmidt, A. L. Harris, L. Old, and V. Cerundolo. 2003. NKT cells enhance CD4+ and CD8+ T cell responses to soluble antigen in vivo through direct interaction with dendritic cells. J Immunol 171:5140-5147.

321. Carnaud, C., D. Lee, O. Donnars, S. H. Park, A. Beavis, Y. Koezuka, and A. Bendelac. 1999. Cutting edge: Cross-talk between cells of the innate immune system: NKT cells rapidly activate NK cells. J Immunol 163:4647-4650.

322. Eberl, G., and H. R. MacDonald. 2000. Selective induction of NK cell proliferation and cytotoxicity by activated NKT cells. Eur J Immunol 30:985-992.

323. Metelitsa, L. S., O. V. Naidenko, A. Kant, H. W. Wu, M. J. Loza, B. Perussia, M. Kronenberg, and R. C. Seeger. 2001. Human NKT cells mediate antitumor cytotoxicity directly by recognizing target cell CD1d with bound ligand or indirectly by producing IL-2 to activate NK cells. J Immunol 167:3114-3122.

324. Chamoto, K., T. Takeshima, A. Kosaka, T. Tsuji, J. Matsuzaki, Y. Togashi, H. Ikeda, and T. Nishimura. 2004. NKT cells act as regulatory cells rather than killer cells during 
activation of NK cell-mediated cytotoxicity by alpha-galactosylceramide in vivo. Immunol Lett 95:5-11.

325. Silk, J. D., I. F. Hermans, U. Gileadi, T. W. Chong, D. Shepherd, M. Salio, B. Mathew, R. R. Schmidt, S. J. Lunt, K. J. Williams, I. J. Stratford, A. L. Harris, and V. Cerundolo. 2004. Utilizing the adjuvant properties of CD1d-dependent NK T cells in T cell-mediated immunotherapy. J Clin Invest 114:1800-1811.

326. Stober, D., I. Jomantaite, R. Schirmbeck, and J. Reimann. 2003. NKT cells provide help for dendritic cell-dependent priming of MHC class I-restricted CD8+ T cells in vivo. J Immunol 170:2540-2548.

327. Burdin, N., L. Brossay, and M. Kronenberg. 1999. Immunization with alphagalactosylceramide polarizes CD1-reactive NK T cells towards Th2 cytokine synthesis. Eur J Immunol 29:2014-2025.

328. Galli, G., S. Nuti, S. Tavarini, L. Galli-Stampino, C. De Lalla, G. Casorati, P. Dellabona, and S. Abrignani. 2003. CD1d-restricted help to B cells by human invariant natural killer T lymphocytes. J Exp Med 197:1051-1057.

329. Hermans, I. F., J. D. Silk, U. Gileadi, S. H. Masri, D. Shepherd, K. J. Farrand, M. Salio, and V. Cerundolo. 2007. Dendritic cell function can be modulated through cooperative actions of TLR ligands and invariant NKT cells. J Immunol 178:27212729.

330. Kitamura, H., A. Ohta, M. Sekimoto, M. Sato, K. Iwakabe, M. Nakui, T. Yahata, H. Meng, T. Koda, S. Nishimura, T. Kawano, M. Taniguchi, and T. Nishimura. 2000. alpha-galactosylceramide induces early B-cell activation through IL-4 production by NKT cells. Cell Immunol 199:37-42.

331. Silk, J. D., M. Salio, B. G. Reddy, D. Shepherd, U. Gileadi, J. Brown, S. H. Masri, P. Polzella, G. Ritter, G. S. Besra, E. Y. Jones, R. R. Schmidt, and V. Cerundolo. 2008. Cutting edge: nonglycosidic CD1d lipid ligands activate human and murine invariant NKT cells. J Immunol 180:6452-6456.

332. Singh, N., S. Hong, D. C. Scherer, I. Serizawa, N. Burdin, M. Kronenberg, Y. Koezuka, and L. Van Kaer. 1999. Cutting edge: activation of NK T cells by CD1d and alpha-galactosylceramide directs conventional $\mathrm{T}$ cells to the acquisition of a Th2 phenotype. J Immunol 163:2373-2377.

333. Barral, P., J. Eckl-Dorna, N. E. Harwood, C. De Santo, M. Salio, P. Illarionov, G. S. Besra, V. Cerundolo, and F. D. Batista. 2008. B cell receptor-mediated uptake of CD1d-restricted antigen augments antibody responses by recruiting invariant NKT cell help in vivo. Proc Natl Acad Sci USA 105:8345-8350.

334. Leadbetter, E. A., M. Brigl, P. Illarionov, N. Cohen, M. C. Luteran, S. Pillai, G. S. Besra, and M. B. Brenner. 2008. NK T cells provide lipid antigen-specific cognate help for B cells. Proc Natl Acad Sci USA 105:8339-8344.

335. Galli, G., P. Pittoni, E. Tonti, C. Malzone, Y. Uematsu, M. Tortoli, D. Maione, G. Volpini, O. Finco, S. Nuti, S. Tavarini, P. Dellabona, R. Rappuoli, G. Casorati, and S. Abrignani. 2007. Invariant NKT cells sustain specific B cell responses and memory. Proc Natl Acad Sci USA 104:3984-3989.

336. Wong, P., and E. G. Pamer. 2003. Feedback regulation of pathogen-specific T cell priming. Immunity 18:499-511.

337. Ludewig, B., W. V. Bonilla, T. Dumrese, and B. Odermatt. 2001. Perforin-independent regulation of dendritic cell homeostasis by CD8 T cells in vivo: implications .... Eur. J. Immunol. 
338. Badovinac, V. P., A. R. Tvinnereim, and J. T. Harty. 2000. Regulation of antigenspecific CD8+ T cell homeostasis by perforin and interferon-gamma. Science 290:1354-1358.

339. Matloubian, M., M. Suresh, A. Glass, M. Galvan, K. Chow, J. K. Whitmire, C. M. Walsh, W. R. Clark, and R. Ahmed. 1999. A role for perforin in downregulating T-cell responses during chronic viral infection. J Virol 73:2527-2536.

340. Sad, S., D. Kägi, and T. R. Mosmann. 1996. Perforin and Fas killing by CD8+ T cells limits their cytokine synthesis and proliferation. J Exp Med 184:1543-1547.

341. Ménasché, G., J. Feldmann, A. Fischer, and G. de Saint Basile. 2005. Primary hemophagocytic syndromes point to a direct link between lymphocyte cytotoxicity and homeostasis. Immunol Rev 203:165-179.

342. Stepp, S. E., P. A. Mathew, M. Bennett, G. de Saint Basile, and V. Kumar. 2000. Perforin: more than just an effector molecule. Immunol Today 21:254-256.

343. Watchmaker, P. B., J. A. Urban, E. Berk, Y. Nakamura, R. B. Mailliard, S. C. Watkins, S. M. van Ham, and P. Kalinski. 2008. Memory CD8+ T cells protect dendritic cells from CTL killing. J Immunol 180:3857-3865.

344. Andrew, K. A., H. M. Simkins, S. Witzel, R. Perret, J. Hudson, I. F. Hermans, D. S. Ritchie, J. Yang, and F. Ronchese. 2008. Dendritic cells treated with lipopolysaccharide up-regulate serine protease inhibitor 6 and remain sensitive to killing by cytotoxic T lymphocytes in vivo. J Immunol 181:8356-8362.

345. Guarda, G., M. Hons, S. Soriano, A. Huang, R. Polley, A. Martín-Fontecha, J. Stein, R. Germain, A. Lanzavecchia, and F. Sallusto. 2007. L-selectin-negative CCR7effector and memory CD8+ T cells enter reactive lymph nodes and kill dendritic cells. Nat Immunol 8:743-752.

346. Platzer, C., G. Richter, K. Uberla, H. Hock, T. Diamantstein, and T. Blankenstein. 1992. Interleukin-4-mediated tumor suppression in nude mice involves interferongamma. Eur J Immunol 22:1729-1733.

347. Lee, A., K. J. Farrand, N. Dickgreber, C. M. Hayman, S. Jürs, I. F. Hermans, and G. F. Painter. 2006. Novel synthesis of alpha-galactosyl-ceramides and confirmation of their powerful NKT cell agonist activity. Carbohydr Res 341:2785-2798.

348. Lugade, A. A., J. P. Moran, S. A. Gerber, R. C. Rose, J. G. Frelinger, and E. M. Lord. 2005. Local radiation therapy of B16 melanoma tumors increases the generation of tumor antigen-specific effector cells that traffic to the tumor. J Immunol 174:75167523.

349. Dalton, D. K., S. Pitts-Meek, S. Keshav, I. S. Figari, A. Bradley, and T. A. Stewart. 1993. Multiple defects of immune cell function in mice with disrupted interferongamma genes. Science 259:1739-1742.

350. Chen, Y. H., N. M. Chiu, M. Mandal, N. Wang, and C. R. Wang. 1997. Impaired $\mathrm{NK} 1+\mathrm{T}$ cell development and early IL-4 production in CD1-deficient mice. Immunity 6:459-467.

351. Hogquist, K. A., S. C. Jameson, W. R. Heath, J. L. Howard, M. J. Bevan, and F. R. Carbone. 1994. T cell receptor antagonist peptides induce positive selection. Cell 76:17-27.

352. Garrigan, K., P. Moroni-Rawson, C. McMurray, I. Hermans, N. Abernethy, J. Watson, and F. Ronchese. 1996. Functional comparison of spleen dendritic cells and dendritic cells cultured in vitro from bone marrow precursors. Blood 88:3508-3512. 
353. Faulkner, L., G. Buchan, L. Slobbe, E. Lockhart, J. Wales, M. Wilson, and M. Baird. 2003. Influenza hemagglutinin peptides fused to interferon gamma and encapsulated in liposomes protects mice against influenza infection. Vaccine 21:932-939.

354. Belz, G. T., W. Xie, J. D. Altman, and P. C. Doherty. 2000. A previously unrecognized $\mathrm{H}-2 \mathrm{D}(\mathrm{b})$-restricted peptide prominent in the primary influenza A virus-specific CD8(+) T-cell response is much less apparent following secondary challenge. $J$ Virol 74:34863493.

355. Belz, G. T., W. Xie, and P. C. Doherty. 2001. Diversity of epitope and cytokine profiles for primary and secondary influenza a virus-specific CD8+ T cell responses. $J$ Immunol 166:4627-4633.

356. Flynn, K. J., G. T. Belz, J. D. Altman, R. Ahmed, D. L. Woodland, and P. C. Doherty. 1998. Virus-specific CD8+ T cells in primary and secondary influenza pneumonia. Immunity 8:683-691.

357. Marshall, D. R., S. J. Turner, G. T. Belz, S. Wingo, S. Andreansky, M. Y. Sangster, J. M. Riberdy, T. Liu, M. Tan, and P. C. Doherty. 2001. Measuring the diaspora for virus-specific CD8+ T cells. Proc Natl Acad Sci USA 98:6313-6318.

358. Spackman, E., D. A. Senne, T. J. Myers, L. L. Bulaga, L. P. Garber, M. L. Perdue, K. Lohman, L. T. Daum, and D. L. Suarez. 2002. Development of a real-time reverse transcriptase PCR assay for type A influenza virus and the avian $\mathrm{H} 5$ and $\mathrm{H} 7$ hemagglutinin subtypes. J Clin Microbiol 40:3256-3260.

359. Brimnes, M. K., L. Bonifaz, R. M. Steinman, and T. M. Moran. 2003. Influenza virusinduced dendritic cell maturation is associated with the induction of strong $\mathrm{T}$ cell immunity to a coadministered, normally nonimmunogenic protein. $J$ Exp Med 198:133-144.

360. Hermans, I. F., J. D. Silk, J. Yang, M. J. Palmowski, U. Gileadi, C. McCarthy, M. Salio, F. Ronchese, and V. Cerundolo. 2004. The VITAL assay: a versatile fluorometric technique for assessing CTL- and NKT-mediated cytotoxicity against multiple targets in vitro and in vivo. J Immunol Methods 285:25-40.

361. Motulsky, H. J. 2003. Prism 4 Statistics Guide-Statistical analyses for laboratory and clinical researchers. GraphPad Software Inc., San Diego, CA, USA.

362. Ashcroft, A. J., S. M. Cruickshank, P. I. Croucher, M. J. Perry, S. Rollinson, J. M. Lippitt, J. A. Child, C. Dunstan, P. J. Felsburg, G. J. Morgan, and S. R. Carding. 2003. Colonic dendritic cells, intestinal inflammation, and T cell-mediated bone destruction are modulated by recombinant osteoprotegerin. Immunity 19:849-861.

363. Nopora, A., and T. Brocker. 2002. Bcl-2 controls dendritic cell longevity in vivo. $J$ Immunol 169:3006-3014.

364. Ludewig, B., W. V. Bonilla, T. Dumrese, B. Odermatt, R. M. Zinkernagel, and H. Hengartner. 2001. Perforin-independent regulation of dendritic cell homeostasis by CD8(+) T cells in vivo: implications for adaptive immunotherapy. Eur J Immunol 31:1772-1779.

365. Doherty, P. C., and J. P. Christensen. 2000. Accessing complexity: the dynamics of virus-specific T cell responses. Annu Rev Immunol 18:561-592.

366. Topham, D. J., R. A. Tripp, and P. C. Doherty. 1997. CD8+ T cells clear influenza virus by perforin or Fas-dependent processes. J Immunol 159:5197-5200.

367. García-Sastre, A., R. K. Durbin, H. Zheng, P. Palese, R. Gertner, D. E. Levy, and J. E. Durbin. 1998. The role of interferon in influenza virus tissue tropism. $J$ Virol 72:85508558. 
368. Bengsch, B., H. C. Spangenberg, N. Kersting, C. Neumann-Haefelin, E. Panther, F. von Weizsäcker, H. E. Blum, H. Pircher, and R. Thimme. 2007. Analysis of CD127 and KLRG1 expression on hepatitis $\mathrm{C}$ virus-specific CD8+ T cells reveals the existence of different memory T-cell subsets in the peripheral blood and liver. $J$ Virol 81:945-953.

369. Joshi, N. S., W. Cui, A. Chandele, H. K. Lee, D. R. Urso, J. Hagman, L. Gapin, and S. M. Kaech. 2007. Inflammation directs memory precursor and short-lived effector CD8(+) T cell fates via the graded expression of T-bet transcription factor. Immunity 27:281-295.

370. Sarkar, S., V. Kalia, W. N. Haining, B. T. Konieczny, S. Subramaniam, and R. Ahmed. 2008. Functional and genomic profiling of effector CD8 T cell subsets with distinct memory fates. J Exp Med 205:625-640.

371. Thimme, R., V. Appay, M. Koschella, E. Panther, E. Roth, A. D. Hislop, A. B. Rickinson, S. L. Rowland-Jones, H. E. Blum, and H. Pircher. 2005. Increased expression of the NK cell receptor KLRG1 by virus-specific CD8 T cells during persistent antigen stimulation. J Virol 79:12112-12116.

372. Price, G. E., L. Huang, R. Ou, M. Zhang, and D. Moskophidis. 2005. Perforin and Fas cytolytic pathways coordinately shape the selection and diversity of CD8+-T-cell escape variants of influenza virus. J Virol 79:8545-8559.

373. Zajac, A. J., J. N. Blattman, K. Murali-Krishna, D. J. Sourdive, M. Suresh, J. D. Altman, and R. Ahmed. 1998. Viral immune evasion due to persistence of activated T cells without effector function. $J$ Exp Med 188:2205-2213.

374. Liu, B., I. Mori, M. J. Hossain, L. Dong, Z. Chen, and Y. Kimura. 2003. Local immune responses to influenza virus infection in mice with a targeted disruption of perforin gene. Microb Pathog 34:161-167.

375. Sambhara, S., I. Switzer, A. Kurichh, R. Miranda, L. Urbanczyk, O. James, B. Underdown, M. Klein, and D. Burt. 1998. Enhanced antibody and cytokine responses to influenza viral antigens in perforin-deficient mice. Cell Immunol 187:13-18.

376. Badovinac, V. P., S. E. Hamilton, and J. T. Harty. 2003. Viral infection results in massive CD8+ T cell expansion and mortality in vaccinated perforin-deficient mice. Immunity 18:463-474.

377. Storm, P., C. Bartholdy, M. R. Sørensen, J. P. Christensen, and A. R. Thomsen. 2006. Perforin-deficient CD8+ T cells mediate fatal lymphocytic choriomeningitis despite impaired cytokine production. J Virol 80:1222-1230.

378. Kägi, D., B. Odermatt, and T. W. Mak. 1999. Homeostatic regulation of CD8+ T cells by perforin. Eur J Immunol 29:3262-3272.

379. Christensen, J. E., D. Wodarz, J. P. Christensen, and A. R. Thomsen. 2004. Perforin and IFN-gamma do not significantly regulate the virus-specific CD8+ T cell response in the absence of antiviral effector activity. Eur J Immunol 34:1389-1394.

380. Wherry, E. J., J. N. Blattman, K. Murali-Krishna, R. van der Most, and R. Ahmed. 2003. Viral persistence alters CD8 T-cell immunodominance and tissue distribution and results in distinct stages of functional impairment. $J$ Virol 77:4911-4927.

381. Chen, W., J. R. Bennink, P. A. Morton, and J. W. Yewdell. 2002. Mice deficient in perforin, CD4+ T cells, or CD28-mediated signaling maintain the typical immunodominance hierarchies of CD8+ T-cell responses to influenza virus. $J$ Virol 76:10332-10337. 
382. Chen, W., K. Pang, K. A. Masterman, G. Kennedy, S. Basta, N. Dimopoulos, F. Hornung, M. J. Smyth, J. R. Bennink, and J. W. Yewdell. 2004. Reversal in the immunodominance hierarchy in secondary CD8+ T cell responses to influenza A virus: roles for cross-presentation and lysis-independent immunodomination. $J$ Immunol 173:5021-5027.

383. Legge, K. L., and T. J. Braciale. 2003. Accelerated migration of respiratory dendritic cells to the regional lymph nodes is limited to the early phase of pulmonary infection. Immunity 18:265-277.

384. Masopust, D., S. J. Ha, V. Vezys, and R. Ahmed. 2006. Stimulation history dictates memory CD8 T cell phenotype: implications for prime-boost vaccination. J Immunol 177:831-839.

385. Wherry, E. J., S. J. Ha, S. M. Kaech, W. N. Haining, S. Sarkar, V. Kalia, S. Subramaniam, J. N. Blattman, D. L. Barber, and R. Ahmed. 2007. Molecular signature of CD8+ T cell exhaustion during chronic viral infection. Immunity 27:670-684.

386. Voehringer, D., C. Blaser, P. Brawand, D. H. Raulet, T. Hanke, and H. Pircher. 2001. Viral infections induce abundant numbers of senescent CD8 T cells. J Immunol 167:4838-4843.

387. Barber, D. L., E. J. Wherry, D. Masopust, B. Zhu, J. P. Allison, A. H. Sharpe, G. J. Freeman, and R. Ahmed. 2006. Restoring function in exhausted CD8 T cells during chronic viral infection. Nature 439:682-687.

388. Knight, D. A., and S. T. Holgate. 2003. The airway epithelium: structural and functional properties in health and disease. Respirology 8:432-446.

389. Joshi, N. S., and S. M. Kaech. 2008. Effector CD8 T cell development: a balancing act between memory cell potential and terminal differentiation. J Immunol 180:1309-1315.

390. Kallies, A. 2008. Distinct regulation of effector and memory T-cell differentiation. Immunol Cell Biol 86:325-332.

391. Bezbradica, J. S., A. K. Stanic, N. Matsuki, H. Bour-Jordan, J. A. Bluestone, J. W. Thomas, D. Unutmaz, L. Van Kaer, and S. Joyce. 2005. Distinct roles of dendritic cells and B cells in Va14Ja18 natural T cell activation in vivo. J Immunol 174:4696-4705.

392. Schmieg, J., G. Yang, R. W. Franck, N. Van Rooijen, and M. Tsuji. 2005. Glycolipid presentation to natural killer T cells differs in an organ-dependent fashion. Proc Natl Acad Sci USA 102:1127-1132.

393. Mieza, M. A., T. Itoh, J. Q. Cui, Y. Makino, T. Kawano, K. Tsuchida, T. Koike, T. Shirai, H. Yagita, A. Matsuzawa, H. Koseki, and M. Taniguchi. 1996. Selective reduction of $\mathrm{V}$ alpha 14+ NK T cells associated with disease development in autoimmune-prone mice. J Immunol 156:4035-4040.

394. Yang, J. Q., V. Saxena, H. Xu, L. Van Kaer, C. R. Wang, and R. R. Singh. 2003. Repeated alpha-galactosylceramide administration results in expansion of NK T cells and alleviates inflammatory dermatitis in MRL-lpr/lpr mice. J Immunol 171:44394446.

395. Leite-de-Moraes, M. C., A. Herbelin, C. Gouarin, Y. Koezuka, E. Schneider, and M. Dy. 2000. Fas/Fas ligand interactions promote activation-induced cell death of NK T lymphocytes. J Immunol 165:4367-4371.

396. Simons, F. E., A. J. Frew, I. J. Ansotegui, B. S. Bochner, D. B. Golden, F. D. Finkelman, D. Y. Leung, J. Lotvall, G. Marone, D. D. Metcalfe, U. Müller, L. J. Rosenwasser, H. A. Sampson, L. B. Schwartz, M. van Hage, and A. F. Walls. 2007. 
Risk assessment in anaphylaxis: current and future approaches. J Allergy Clin Immunol 120:S2-24.

397. Sampson, H. A., A. Muñoz-Furlong, R. L. Campbell, N. F. Adkinson, S. A. Bock, A. Branum, S. G. Brown, C. A. Camargo, R. Cydulka, S. Galli, J. Gidudu, R. S. Gruchalla, A. D. Harlor, D. L. Hepner, L. M. Lewis, P. L. Lieberman, D. D. Metcalfe, R. O'Connor, A. Muraro, A. Rudman, C. Schmitt, D. Scherrer, F. E. Simons, S. Thomas, J. P. Wood, and W. W. Decker. 2006. Second symposium on the definition and management of anaphylaxis: summary report--Second National Institute of Allergy and Infectious Disease/Food Allergy and Anaphylaxis Network symposium. $J$ Allergy Clin Immunol 117:391-397.

398. Galli, S., S. Nakae, and M. Tsai. 2005. Mast cells in the development of adaptive immune responses. Nat Immunol 6:135-142.

399. Dombrowicz, D., V. Flamand, I. Miyajima, J. V. Ravetch, S. J. Galli, and J. P. Kinet. 1997. Absence of Fc epsilonRI alpha chain results in upregulation of Fc gammaRIIIdependent mast cell degranulation and anaphylaxis. Evidence of competition between Fc epsilonRI and Fc gammaRIII for limiting amounts of FcR beta and gamma chains. $J$ Clin Invest 99:915-925.

400. Finkelman, F. 2007. Anaphylaxis: Lessons from mouse models. J Allergy Clin Immunol 120:506-515.

401. Miyajima, I., D. Dombrowicz, T. R. Martin, J. V. Ravetch, J. P. Kinet, and S. J. Galli. 1997. Systemic anaphylaxis in the mouse can be mediated largely through IgG1 and Fc gammaRIII. Assessment of the cardiopulmonary changes, mast cell degranulation, and death associated with active or IgE- or IgG1-dependent passive anaphylaxis. J Clin Invest 99:901-914.

402. Tsujimura, Y., K. Obata, K. Mukai, H. Shindou, M. Yoshida, H. Nishikado, Y. Kawano, Y. Minegishi, T. Shimizu, and H. Karasuyama. 2008. Basophils play a pivotal role in immunoglobulin-G-mediated but not immunoglobulin-E-mediated systemic anaphylaxis. Immunity 28:581-589.

403. Prussin, C., and D. D. Metcalfe. 2006. 5. IgE, mast cells, basophils, and eosinophils. $J$ Allergy Clin Immunol 117:S450-456.

404. Ashany, D., A. Savir, N. Bhardwaj, and K. B. Elkon. 1999. Dendritic Cells Are Resistant to Apoptosis Through the Fas (CD95/APO-1) Pathway 1. J Immunol 163:5303-5311.

405. Kitamura, H., K. Iwakabe, T. Yahata, S. Nishimura, A. Ohta, Y. Ohmi, M. Sato, K. Takeda, K. Okumura, L. Van Kaer, T. Kawano, M. Taniguchi, and T. Nishimura. 1999. The natural killer T (NKT) cell ligand alpha-galactosylceramide demonstrates its immunopotentiating effect by inducing interleukin (IL)-12 production by dendritic cells and IL-12 receptor expression on NKT cells. J Exp Med 189:1121-1128.

406. Parekh, V. V., M. T. Wilson, D. Olivares-Villagómez, A. K. Singh, L. Wu, C. R. Wang, S. Joyce, and L. Van Kaer. 2005. Glycolipid antigen induces long-term natural killer T cell anergy in mice. J Clin Invest 115:2572-2583.

407. Uldrich, A. P., N. Y. Crowe, K. Kyparissoudis, D. G. Pellicci, Y. Zhan, A. M. Lew, P. Bouillet, A. Strasser, M. J. Smyth, and D. I. Godfrey. 2005. NKT cell stimulation with glycolipid antigen in vivo: costimulation-dependent expansion, Bim-dependent contraction, and hyporesponsiveness to further antigenic challenge. J Immunol 175:3092-3101. 
408. Galli, S., M. Tsai, and A. Piliponsky. 2008. The development of allergic inflammation. Nature 454:445-454.

409. Li, X. M., D. Serebrisky, S. Y. Lee, C. K. Huang, L. Bardina, B. H. Schofield, J. S. Stanley, A. W. Burks, G. A. Bannon, and H. A. Sampson. 2000. A murine model of peanut anaphylaxis: T- and B-cell responses to a major peanut allergen mimic human responses. J Allergy Clin Immunol 106:150-158.

410. McCaskill, A. C., C. S. Hosking, and D. J. Hill. 1984. Anaphylaxis following intranasal challenge of mice sensitized with ovalbumin. Immunology 51:669-677.

411. Malveaux, F. J., M. C. Conroy, N. F. Adkinson, and L. M. Lichtenstein. 1978. IgE receptors on human basophils. Relationship to serum IgE concentration. J Clin Invest 62:176-181.

412. Lantz, C. S., M. Yamaguchi, H. C. Oettgen, I. M. Katona, I. Miyajima, J. P. Kinet, and S. J. Galli. 1997. IgE regulates mouse basophil Fc epsilon RI expression in vivo. J Immunol 158:2517-2521.

413. Karasuyama, H., K. Mukai, Y. Tsujimura, and K. Obata. 2009. Newly discovered roles for basophils: a neglected minority gains new respect. Nat Rev Immunol 9:9-13.

414. Kojima, T., K. Obata, K. Mukai, S. Sato, T. Takai, Y. Minegishi, and H. Karasuyama. 2007. Mast cells and basophils are selectively activated in vitro and in vivo through CD200R3 in an IgE-independent manner. J Immunol 179:7093-7100.

415. Obata, K., K. Mukai, Y. Tsujimura, K. Ishiwata, Y. Kawano, Y. Minegishi, N. Watanabe, and H. Karasuyama. 2007. Basophils are essential initiators of a novel type of chronic allergic inflammation. Blood 110:913-920.

416. Belz, G. T., C. M. Smith, D. Eichner, K. Shortman, G. Karupiah, F. R. Carbone, and W. R. Heath. 2004. Cutting edge: conventional CD8 alpha+ dendritic cells are generally involved in priming CTL immunity to viruses. J Immunol 172:1996-2000.

417. Lin, M. L., Y. Zhan, A. I. Proietto, S. Prato, L. Wu, W. R. Heath, J. A. Villadangos, and A. M. Lew. 2008. Selective suicide of cross-presenting CD8+ dendritic cells by cytochrome c injection shows functional heterogeneity within this subset. Proc Natl Acad Sci USA 105:3029-3034.

418. Iyoda, T., S. Shimoyama, K. Liu, Y. Omatsu, Y. Akiyama, Y. Maeda, K. Takahara, R. M. Steinman, and K. Inaba. 2002. The CD8+ dendritic cell subset selectively endocytoses dying cells in culture and in vivo. J Exp Med 195:1289-1302.

419. Schulz, O., and C. Reis E Sousa. 2002. Cross-presentation of cell-associated antigens by CD8alpha+ dendritic cells is attributable to their ability to internalize dead cells. Immunology 107:183-189.

420. Qiu, C. H., Y. Miyake, H. Kaise, H. Kitamura, O. Ohara, and M. Tanaka. 2009. Novel subset of CD8 $\{$ alpha $\}+$ dendritic cells localized in the marginal zone is responsible for tolerance to cell-associated antigens. J Immunol 182:4127-4136.

421. Douillard, P., P. Stoitzner, C. H. Tripp, V. Clair-Moninot, S. Ait-Yahia, A. D. McLellan, A. Eggert, N. Romani, and S. Saeland. 2005. Mouse lymphoid tissue contains distinct subsets of langerin/CD207 dendritic cells, only one of which represents epidermal-derived Langerhans cells. J Invest Dermatol 125:983-994.

422. Takahara, K., Y. Omatsu, Y. Yashima, Y. Maeda, S. Tanaka, T. Iyoda, B. E. Clausen, K. Matsubara, J. Letterio, R. M. Steinman, Y. Matsuda, K. Inaba, and B. Clusen. 2002. Identification and expression of mouse Langerin (CD207) in dendritic cells. Int Immunol 14:433-444. 
423. McLellan, A. D., M. Kapp, A. Eggert, C. Linden, U. Bommhardt, E. B. Bröcker, U. Kämmerer, and E. Kämpgen. 2002. Anatomic location and T-cell stimulatory functions of mouse dendritic cell subsets defined by CD4 and CD8 expression. Blood 99:2084-2093.

424. O'Keeffe, M. A., H. Hochrein, D. Vremec, I. Caminschi, J. L. Miller, E. M. Anders, L. Wu, M. H. Lahoud, S. Henri, B. Scott, P. Hertzog, L. Tatarczuch, and K. Shortman. 2002. Mouse plasmacytoid cells: long-lived cells, heterogeneous in surface phenotype and function, that differentiate into CD8(+) dendritic cells only after microbial stimulus. J Exp Med 196:1307-1319.

425. Vincent, M., D. Leslie, J. Gumperz, X. Xiong, E. P. Grant, and M. B. Brenner. 2002. CD1-dependent dendritic cell instruction. Nat Immunol 3:1163-1168.

426. Stanic, A. K., R. Shashidharamurthy, J. S. Bezbradica, N. Matsuki, Y. Yoshimura, S. Miyake, E. Y. Choi, T. D. Schell, L. Van Kaer, S. S. Tevethia, D. C. Roopenian, T. Yamamura, and S. Joyce. 2003. Another view of T cell antigen recognition: cooperative engagement of glycolipid antigens by Va14Ja18 natural TCR. J Immunol 171:4539-4551.

427. Wallach, D., E. E. Varfolomeev, N. L. Malinin, Y. V. Goltsev, A. V. Kovalenko, and M. P. Boldin. 1999. Tumor necrosis factor receptor and Fas signaling mechanisms. Annu Rev Immunol 17:331-367.

428. Idoyaga, J., N. Suda, K. Suda, C. G. Park, and R. M. Steinman. 2009. Antibody to Langerin/CD207 localizes large numbers of CD8alpha+ dendritic cells to the marginal zone of mouse spleen. Proc Natl Acad Sci USA 106:1524-1529.

429. Hildeman, D., T. Jorgensen, J. Kappler, and P. Marrack. 2007. Apoptosis and the homeostatic control of immune responses. Curr Opin Immunol 19:516-521.

430. De Trez, C., B. Pajak, M. Brait, N. Glaichenhaus, J. Urbain, M. Moser, G. Lauvau, and E. Muraille. 2005. TLR4 and Toll-IL-1 receptor domain-containing adapter-inducing IFN-beta, but not MyD88, regulate Escherichia coli-induced dendritic cell maturation and apoptosis in vivo. J Immunol 175:839-846.

431. Efron, P. A., A. Martins, D. Minnich, K. Tinsley, R. Ungaro, F. R. Bahjat, R. Hotchkiss, M. Clare-Salzler, and L. L. Moldawer. 2004. Characterization of the systemic loss of dendritic cells in murine lymph nodes during polymicrobial sepsis. $J$ Immunol 173:3035-3043.

432. Sponaas, A. M., E. T. Cadman, C. Voisine, V. Harrison, A. Boonstra, A. O'Garra, and J. Langhorne. 2006. Malaria infection changes the ability of splenic dendritic cell populations to stimulate antigen-specific T cells. J Exp Med 203:1427-1433.

433. Sundquist, M., and M. J. Wick. 2009. Salmonella induces death of CD8alpha(+) dendritic cells but not CD11c(int)CD11b(+) inflammatory cells in vivo via MyD88 and TNFR1. J Leukoc Biol 85:225-234.

434. Stanic, A. K., R. Shashidharamurthy, J. S. Bezbradica, N. Matsuki, Y. Yoshimura, S. Miyake, E. Y. Choi, T. D. Schell, L. Van Kaer, S. S. Tevethia, D. C. Roopenian, T. Yamamura, and S. Joyce. 2003. Another view of T cell antigen recognition: cooperative engagement of glycolipid antigens by Va14Ja18 natural T(iNKT) cell receptor [corrected]. J Immunol 171:4539-4551.

435. Shimizu, K., Y. Kurosawa, M. Taniguchi, R. M. Steinman, and S. Fujii. 2007. Crosspresentation of glycolipid from tumor cells loaded with alpha-galactosylceramide leads to potent and long-lived T cell mediated immunity via dendritic cells. $J$ Exp Med 204:2641-2653. 
436. Wu, D. Y., N. H. Segal, S. Sidobre, M. Kronenberg, and P. B. Chapman. 2003. Crosspresentation of disialoganglioside GD3 to natural killer T cells. J Exp Med 198:173181.

437. Locksley, R. M., N. Killeen, and M. J. Lenardo. 2001. The TNF and TNF receptor superfamilies: integrating mammalian biology. Cell 104:487-501.

438. Smyth, M. J., and J. D. Sedgwick. 1998. Delayed kinetics of tumor necrosis factormediated bystander lysis by peptide-specific CD8+ cytotoxic T lymphocytes. Eur J Immunol 28:4162-4169.

439. Liu, Z. G., H. Hsu, D. V. Goeddel, and M. Karin. 1996. Dissection of TNF receptor 1 effector functions: JNK activation is not linked to apoptosis while NF-kappaB activation prevents cell death. Cell 87:565-576.

440. Monastra, G., A. Cabrelle, A. Zambon, A. Rosato, B. Macino, D. Collavo, and P. Zanovello. 1996. Membrane form of TNF alpha induces both cell lysis and apoptosis in susceptible target cells. Cell Immunol 171:102-110.

441. Perez, C., I. Albert, K. DeFay, N. Zachariades, L. Gooding, and M. Kriegler. 1990. A nonsecretable cell surface mutant of tumor necrosis factor (TNF) kills by cell-to-cell contact. Cell 63:251-258.

442. Makarenkova, V., A. K. Chakrabarti, J. A. Liberatore, P. Popovic, G. Lu, S. Watkins, and N. L. Vujanovic. 2005. Dendritic cells and natural killer cells interact via multiple TNF family molecules. J Leukoc Biol 77:408-413.

443. Bialecki, E., C. Paget, J. Fontaine, M. Capron, F. Trottein, and C. Faveeuw. 2009. Role of marginal zone B lymphocytes in invariant NKT cell activation. J Immunol 182:6105-6113.

444. Metkar, S. S., C. Menaa, J. Pardo, B. Wang, R. Wallich, M. Freudenberg, S. Kim, S. M. Raja, L. Shi, M. M. Simon, and C. J. Froelich. 2008. Human and mouse granzyme A induce a proinflammatory cytokine response. Immunity 29:720-733.

445. Peng, S. L., J. Moslehi, M. E. Robert, and J. Craft. 1998. Perforin protects against autoimmunity in lupus-prone mice. J Immunol 160:652-660.

446. Spaner, D., K. Raju, B. Rabinovich, and R. G. Miller. 1999. A role for perforin in activation-induced $\mathrm{T}$ cell death in vivo: increased expansion of allogeneic perforindeficient T cells in SCID mice. J Immunol 162:1192-1199.

447. Spielman, J., R. K. Lee, and E. R. Podack. 1998. Perforin/Fas-ligand double deficiency is associated with macrophage expansion and severe pancreatitis. J Immunol 161:70637070.

448. Stepp, S. E., R. Dufourcq-Lagelouse, F. Le Deist, S. Bhawan, S. Certain, P. A. Mathew, J. I. Henter, M. Bennett, A. Fischer, G. de Saint Basile, and V. Kumar. 1999. Perforin gene defects in familial hemophagocytic lymphohistiocytosis. Science 286:1957-1959.

449. de Saint Basile, G., and A. Fischer. 2001. The role of cytotoxicity in lymphocyte homeostasis. Curr Opin Immunol 13:549-554.

450. Thedrez, A., C. De Lalla, S. Allain, L. Zaccagnino, S. Sidobre, C. Garavaglia, G. Borsellino, P. Dellabona, M. Bonneville, E. Scotet, and G. Casorati. 2007. CD4 engagement by CD1d potentiates activation of CD4+ invariant NKT cells. Blood 110:251-258.

451. Chen, X., X. Wang, G. S. Besra, and J. Gumperz. 2007. Modulation of CD1drestricted NKT cell responses by CD4. J Leukoc Biol 82:1455-1465. 
452. Mirandola, P., C. Ponti, G. Gobbi, I. Sponzilli, M. Vaccarezza, L. Cocco, G. Zauli, P. Secchiero, F. A. Manzoli, and M. Vitale. 2004. Activated human NK and CD8+ T cells express both TNF-related apoptosis-inducing ligand (TRAIL) and TRAIL receptors but are resistant to TRAIL-mediated cytotoxicity. Blood 104:2418-2424.

453. Stranges, P. B., J. Watson, C. J. Cooper, C. M. Choisy-Rossi, A. C. Stonebraker, R. A. Beighton, H. Hartig, J. P. Sundberg, S. Servick, G. Kaufmann, P. J. Fink, and A. V. Chervonsky. 2007. Elimination of antigen-presenting cells and autoreactive T cells by Fas contributes to prevention of autoimmunity. Immunity 26:629-641.

454. Brincks, E. L., A. Katewa, T. A. Kucaba, T. S. Griffith, and K. L. Legge. 2008. CD8 T cells utilize TRAIL to control influenza virus infection. J Immunol 181:4918-4925.

455. Nagata, S., and T. Suda. 1995. Fas and Fas ligand: lpr and gld mutations. Immunol Today 16:39-43.

456. Shevach, E. M. 2009. Mechanisms of foxp3+ T regulatory cell-mediated suppression. Immunity 30:636-645.

457. Sun, J., R. Madan, C. Karp, and T. J. Braciale. 2009. Effector T cells control lung inflammation during acute influenza virus infection by producing IL-10. Nat Med 15:277-284.

458. Chang, D. H., K. Osman, J. Connolly, A. Kukreja, J. Krasovsky, M. Pack, A. Hutchinson, M. Geller, N. Liu, R. Annable, J. Shay, K. Kirchhoff, N. Nishi, Y. Ando, K. Hayashi, H. Hassoun, R. M. Steinman, and M. V. Dhodapkar. 2005. Sustained expansion of NKT cells and antigen-specific T cells after injection of alpha-galactosylceramide loaded mature dendritic cells in cancer patients. J Exp Med 201:1503-1517.

459. Ishikawa, A., S. Motohashi, E. Ishikawa, H. Fuchida, K. Higashino, M. Otsuji, T. Iizasa, T. Nakayama, M. Taniguchi, and T. Fujisawa. 2005. A phase I study of alphagalactosylceramide (KRN7000)-pulsed dendritic cells in patients with advanced and recurrent non-small cell lung cancer. Clin Cancer Res 11:1910-1917.

460. Nieda, M., M. Okai, A. Tazbirkova, H. Lin, A. Yamaura, K. Ide, R. Abraham, T. Juji, D. J. Macfarlane, and A. J. Nicol. 2004. Therapeutic activation of Valpha24+Vbeta11+ NKT cells in human subjects results in highly coordinated secondary activation of acquired and innate immunity. Blood 103:383-389.

461. Okai, M., M. Nieda, A. Tazbirkova, D. Horley, A. Kikuchi, S. Durrant, T. Takahashi, A. Boyd, R. Abraham, H. Yagita, T. Juji, and A. Nicol. 2002. Human peripheral blood Valpha24+ Vbeta11+ NKT cells expand following administration of alphagalactosylceramide-pulsed dendritic cells. Vox Sang 83:250-253.

462. Uchida, T., S. Horiguchi, Y. Tanaka, H. Yamamoto, N. Kunii, S. Motohashi, M. Taniguchi, T. Nakayama, and Y. Okamoto. 2008. Phase I study of alphagalactosylceramide-pulsed antigen presenting cells administration to the nasal submucosa in unresectable or recurrent head and neck cancer. Cancer Immunol Immunother 57:337-345.

463. Motohashi, S., and T. Nakayama. 2008. Clinical applications of natural killer T cellbased immunotherapy for cancer. Cancer Science 99:638-645.

464. Belz, G. T., S. Bedoui, F. Kupresanin, F. R. Carbone, and W. R. Heath. 2007. Minimal activation of memory CD8+ T cell by tissue-derived dendritic cells favors the stimulation of naive CD8+ T cells. Nat Immunol 8:1060-1066. 


\section{Appendix 1: Publications}

\section{Publications resulting from work presented in this thesis}

Helen M.A. Simkins, Kathryn J. Farrand, Ian F. Hermans and Franca Ronchese. The survival of DC subpopulations in vivo is affected by $\alpha$-GalCer administration. In preparation.

\section{Publications contributed to during the PhD studies}

Kate A. Andrew, Helen M.A. Simkins, Sabine Witzel, Rachel Perret, Jenny Hudson, Ian F. Hermans, David S. Ritchie, Jianping Yang, and Franca Ronchese. Dendritic cells treated with lipopolysaccharide up-regulate serine protease inhibitor 6 and remain sensitive to killing by cytotoxic T lymphocytes in vivo. The journal of immunology, 2008, 181:8356-8362. 